\title{
Optical Conductivity of Colossal Magnetoresistance Manganites.
}

by

Ocean Ripeka Mercier

\begin{abstract}
A thesis
submitted to the Victoria University of Wellington in fulfilment of the requirements for the degree of

Doctor of Philosophy

in Physics
\end{abstract}

Victoria University of Wellington

February 2002 


\begin{abstract}
.
The colossal magnetoresistance manganites are a group of materials whose unusual physical properties are a symptom of strongly interacting electrons and phonons. In order to elucidate some of these electronic and vibrational properties, an infrared optical investigation of manganites with a broad range of physical characteristics has been performed. Temperature-dependent normal incidence reflectivity measurements have been made on two samples of manganites, in the energy range of $60 \mathrm{~cm}^{-1}-50000 \mathrm{~cm}^{-1}$,

1. for $\mathrm{La}_{0.9} \mathrm{Ca}_{0.1} \mathrm{MnO}_{3}$, an insulating ferromagnet ${ }^{1}$, and

2. $\mathrm{La}_{0.735} \mathrm{Ca}_{0.265} \mathrm{MnO}_{3}$, a metallic ferromagnet ${ }^{1}$.

Temperature-dependent ellipsometric reflection measurements were performed in the energy range of $50 \mathrm{~cm}^{-1}-5000 \mathrm{~cm}^{-1}$, on four faces of two samples of structurally anisotropic manganite, probing the

3. $a b$ plane and $c$-axis of $\mathrm{La}_{1.2} \mathrm{Sr}_{1.8} \mathrm{Mn}_{2} \mathrm{O}_{7}$, a metallic ferromagnet ${ }^{1}$, and

4. the $a b$ plane and $c$-axis of $\operatorname{PrSr}_{2} \mathrm{Mn}_{2} \mathrm{O}_{7}$, an insulating antiferromagnet ${ }^{1}$.

The optical conductivity for each of the first two samples has been deduced by a careful Kramers-Kronig analyis of the normal incidence reflectivity. For samples 3. and 4. the optical conductivity has been deduced by inversion of the ellipsometric constants, and a careful subsequent fitting to account for their anisotropy. The transition temperatures and types of magnetic order for all samples have also been characterised by magnetisation measurements.
\end{abstract}

\footnotetext{
${ }^{1}$ These are the low-temperature state properties of the $a b$ plane.
} 
Treatment of the surface is shown to be critical in reflectivity measurements by the observation of hugely contrasting spectra, measured from a polished sample of metallic-like $\mathrm{La}_{0.735} \mathrm{Ca}_{0.265} \mathrm{MnO}_{3}$, before and after annealing.

Several features observed in the measurements, especially for the layered materials, are consistent with the idea that a polaron, or electron-lattice interaction, is hugely important in a description of the electron dynamics of these materials. The correlation between spectral features and the structural and magnetic properties of the materials is investigated, finding that the cause of charge transport modification seen in the metallic-like materials could be explained by either a polaron or localisation due to disorder. 


\section{Preface}

\footnotetext{
"...no one can think more highly of the understanding of women than I do. In my opinion, nature has given them so much that they never find it necessary to use more than half." Jane Austen, Northanger Abbey.
}

This work forms the written component of the research undertaken in the period February 1998 to November 2001, to fulfill the requirements of the Victoria University of Wellington PhD degree. The work presented in this thesis has also been published in the following list of publications:

1. O. Ripeka Mercier, R. G. Buckley, A. Bittar, H. J. Trodahl, E. M. Haines, J. Metson, and Y. Tomioka Annealing effects on the optical conductivity of single crystal $\mathrm{La}_{1-x} \mathrm{Ca}_{x} \mathrm{MnO}_{3},(x=0.1,0.265)$. Phys. Rev. B 64035106 (2001)

2. O. Ripeka Mercier, C. Bernhard, T. Holden, H. J. Trodahl, R. G. Buckley, J. Humliček and G. Balakrishnan Optical Conductivity of Layered $\mathrm{PrSr}_{2} \mathrm{Mn}_{2} \mathrm{O}_{7}$ : Phonon Anomalies and Polaron Signatures. In preparation.

3. O. Ripeka Mercier, C. Bernhard, T. Holden, R. G. Buckley, H. J. Trodahl, J. Humliček and G. Balakrishnan $a b$ plane and $c$-axis Optical Conductivity of $\mathrm{La}_{1.2} \mathrm{Sr}_{1.8} \mathrm{Mn}_{2} \mathrm{O}_{7}$ Ellipsometry Bridges the Gaps. In preparation.

Results herein have been presented on the following occasions: 
1. Invited seminars given at:

(a) Victoria University of Wellington, New Zealand (June 2000).

(b) Imperial College of Science, Technology and Medicine, London, United Kingdom (April 2000).

(c) Industrial Research Limited, Wellington, New Zealand (1999, 1998).

2. Contributed seminars given at:

(a) New Zealand Institute of Physics, Tenth National Conference, Wellington, New Zealand (July 2001).

(b) 25th Annual Condensed Matter Meeting, Marlborough Sounds, New Zealand (March 2001).

(c) American Physical Society March Meeting, Minneapolis, Minnesota, USA (March 2000).

(d) New Zealand Institute of Physics, Ninth National Conference, Wellington, New Zealand (July 1999).

This thesis is mostly concerned with energy, and the electronic and vibrational excitations occuring at those energies. As such, it is important that the reader, from the outset, be conversant in the language of energy used here.

For a start, the terms energy, frequency, and wavelength are all used interchangeably. In describing energy regions, the term wavelength is usually used with reference to the visible part of the spectrum. The term frequency is more commonly used in the low energy (!) part of the infrared region. This choice emerges from the vibrational character of the atoms in the manganite crystal lattice. It seems natural to describe the energies associated with these vibrations as frequencies.

The traditional partitioning of the infrared (IR) region is used extensively in this thesis. The energies between $50 \mathrm{~cm}^{-1}$ and $700 \mathrm{~cm}^{-1}$ are referred to 


\begin{tabular}{|r||r|l|r|}
\hline Wavenumber $\left(\mathrm{cm}^{-1}\right)$ & Energy $(\mathrm{eV})$ & Freq. $\left(10^{12} \mathrm{~Hz}\right)$ & Wavelength $(\mu \mathrm{m})$ \\
\hline \hline 10 & 0.00124 & 0.3 & 1000 \\
200 & 0.0248 & 6 & 50 \\
500 & 0.062 & 15 & 20 \\
1000 & 0.124 & 30 & 10 \\
10000 & 1.24 & 300 & 1 \\
\hline
\end{tabular}

Table 1: Table showing the equivalent energy $(\mathrm{eV})$, frequency $\left(10^{12} \mathrm{~Hz}\right)$ and wavelength $(\mu \mathrm{m})$ for selected values of the wavenumber $\left(\mathrm{cm}^{-1}\right)$.

as the far-infrared (far-IR). That between $700 \mathrm{~cm}^{-1}$ and $7000 \mathrm{~cm}^{-1}$ is referred to as the mid-infrared (mid-IR). Finally, energies between $7000 \mathrm{~cm}^{-1}$ and $13000 \mathrm{~cm}^{-1}$ are taken as the near-infrared (near-IR). The abbreviations in parentheses are used aggressively, and without warning or apology, throughout this thesis.

The energy units of choice are the wavenumber, or $\mathrm{cm}^{-1}$, and are used fairly consistently throughout, except in some sections where the spectral results of other research groups are discussed. The conversions between the frequency and wavelength in their various unit types are given in the above table for selected values of wavenumber.

A couple of final points on mathematical notation now. Firstly, a vector quantity will be represented by emboldening the variable name; for instance $\mathbf{E}_{\mathbf{0}}$. And finally, a complex quantity will usually be denoted as having a tilde on top of the variable name, for example, the dielectric constant, $\tilde{\epsilon}$. The notable exceptions include the reflection coefficients, $r_{s}, r_{p}$ and $\rho$, for which complex values are implicitly assumed. 


\section{Acknowledgments}

This thesis might not have been written, were it not for the ever-abundant belief and encouragement of my supervisors, Prof. Joe Trodahl and Dr. Robert Buckley. I extend the greatest of thanks for their guidance during the course of the research. Bob's impeccably timed suggestions and advice always seemed to coincide with my most needful moments. Joe's affable nature has ensured that the physics has been as enjoyable as it has been hard work. I am grateful to Dr. Christian Bernhard, whose enthusiasm for science is almost tangibly felt by his monstrous work ethic, vivacious demeanour in the lab and willingness to take this greenhorn under his wing for the ellipsometry measurements. I am indebted to Dr. Tony Bittar for help on the visible monochromator system, and very grateful to Dr. Ben Chapman; mentor and lab companion for showing me the where, what and how of the equipment at Industrial Research Ltd. My thanks also to Dr. Todd Holden; mentor and true gentleman, for showing me the where, what and how of the equipment at the Max-Planck Institut in Stuttgart. Technical support from Dr. Larry Carr and Dr. Michael Pilling at the U4IR beamline is also gratefully acknowledged. The visits from over-the-seas of Dr. Esther Haines have been extremely beneficial to the project, Esther providing expertise and insight on almost every theoretical aspect touched on in this thesis. Thanks to her also for the Kramers-Kronig program used. We have also benefitted from discussions with Dr. José Lorenzana, Prof. Neil Ashcroft and Dr. Nicolas Witte. Thanks to the Materials' Physics team at Industrial Research Ltd, especially Dr. Mike Staines, Stephan Rupp, Dr. Donald Pooke, Dr. Nick Strickland, Dave Cochrane and Kay Card for technical expertise in various areas. I appreciated the patience of Dr. Andy Edgar, in 
suffering an extended-term loan of his flow-through cryostat. The work would not have been possible, of course, without the samples provided. Thanks to Dr. Yasuhide Tomioka and Dr. Geetha Balakrishnan for these and useful correspondence thereto related. My thanks also to Dr. Andrzej Golnik for the ellipsometric reduction MATLAB program.

My colleagues have been fun and stimulating to work alongside: thankyou Olly Pantoja, Andreas Engel and Martin Howard. A big Shout-Out to the Hacky Sackers: Jonathon Whittle, Brent Walker, David Stewart, Iain Matcham, Irina Chamritskaya, Hamish Edgar, Nigel Ross, and regular sessions have helped keep mind and body fit, and life in perspective. The cultural diversity of the GreenRoom occupants over the years has made life in the office often as exciting as life outside of it. Thanks especially to Andrea Bubendorfer; I've appreciated your friendship and wisdom. Special thanks to family and friends, especially my parents, for their forbearance of my physical and mental estrangements throughout the course of the project. You are too many to name who have kept me going by your love, support and words of encouragement. However I do thankfully mention Rev. and Mrs Hastings, without whose initial advice, encouragement and prayer, I would not have embarked on this project, and appreciate also their donation of a computer for late-night writing sessions at home. Arohanui ki a tatou katoa. I also acknowledge the Foundation for Research into Science and Technology, for their vision and provision of the Tūāpapa Pūtaiao Māori Fellowship. Last, and most, I thank Jesus Christ, my Saviour, who is a light in dark places. May this work bring glory to Him.

For as the heavens are higher than the earth, so are my ways higher than your ways, and my thoughts than your thoughts. Isaiah 55:9. 


\section{Contents}

Preface v v

$\begin{array}{ll}\text { Acknowledgments } & \text { ix }\end{array}$

1 CMR manganites. $\quad 1$

1.1 Conductivity of manganites. . . . . . . . . . . . 11

2 Background Theory. 17

2.1 Light and its interaction with a medium. . . . . . . . 17

2.1.1 Maxwell's equations. . . . . . . . . . . . 17

2.1.2 Behaviour of $\mathbf{E}$ at an interface. . . . . . . . . 18

2.2 Ellipsometric measurement of $\tilde{\epsilon}(\omega) \ldots \ldots \ldots \ldots$

2.2 .1 Light polarisation description . . . . . . . . . 20 20

2.2 .2 Rotating analyser ellipsometer . . . . . . . . . . . 23

2.2 .3 Reduction of ellipsometric constants. . . . . . . . . 24

2.2.4 Deduction of $c$-axis conductivity with $a b$ plane measurements. . . . . . . . . . . . . . . 2 27

2.3 Normal Incidence reflectance measurement of $\tilde{\epsilon}(\omega) \ldots \ldots 30$

2.3.1 Deduction of $\tilde{\epsilon}(\omega)$ from $R(\omega) \ldots \ldots \ldots$. . . . . 30

2.3 .2 Sum rules . . . . . . . . . . . . . . . . . 33

2.3.3 Reflectance extrapolations. . . . . . . . . . . . . 33

2.4 Optical response of a harmonic oscillator. . . . . . . . . . 35

2.4 .1 The bound charge response . . . . . . . . . 35

2.4 .2 The Drude response. . . . . . . . . . . . . 37 
2.4.3 Phonon oscillators ............... . . 39

3 Method. $\quad 41$

3.1 Fourier-Transform Spectroscopy. . . . . . . . . . . . . . . . . . 42

3.2 Normal Incidence Reflectance. . . . . . . . . . . . . . . . . . . 44

3.2.1 Spectrometer resources. . . . . . . . . . . . . . 44

3.2 .2 Optical bench. . . . . . . . . . . . . . . . . 44

3.2.3 From transmission to reflection. . . . . . . . . . 46

3.2.4 Temperature-dependent measurements. . . . . . . . . . 50

3.2.5 Grating Monochromator. . . . . . . . . . . . . 50

3.3 Ellipsometry. . . . . . . . . . . . . . . . . 52

3.3.1 Spectrometer Resources. . . . . . . . . . . . . . 54

3.3 .2 Optical bench. . . . . . . . . . . . . . . . . 55

3.3.3 Sample mounting. . . . . . . . . . . . . . . 55

3.3.4 Temperature-dependent measurements. . . . . . . . . 56

3.4 Ellipsometry at Brookhaven . . . . . . . . . . . . . . 56

3.4 .1 Optical bench. . . . . . . . . . . . . . 56

3.4 .2 Beamline characteristics . . . . . . . . . . 57

3.5 Sample Preparation. . . . . . . . . . . . . . . . . 58

3.5.1 Preparing surfaces for measurement. . . . . . . . . 58

3.5.2 Pseudocubic $\mathrm{La}_{1-x} \mathrm{Ca}_{x} \mathrm{MnO}_{3}$. . . . . . . . . . . . . 59

3.5.3 Layered (La/Pr) ${ }_{2-2 x} \mathrm{Sr}_{1+2 x} \mathrm{Mn}_{2} \mathrm{O}_{7} \ldots \ldots . . . . .66$

$\begin{array}{lll}4 & \text { On reflectivity matters } & 67\end{array}$

4.1 Pseudocubic $\mathrm{La}_{0.9} \mathrm{Ca}_{0.1} \mathrm{MnO}_{3}$. . . . . . . . . . . . . . . . 68

4.1.1 Reflectivity spectra . . . . . . . . . . . 68

4.1 .2 Conductivity spectra . . . . . . . . . . 70

4.2 Pseudocubic $\mathrm{La}_{0.735} \mathrm{Ca}_{0.265} \mathrm{MnO}_{3}$. . . . . . . . . . . . . . 72

4.2 .1 Extrapolations . . . . . . . . . . . . . . . 73

4.2.2 Annealing effects on $R(\omega)$ and $\sigma_{\kappa \kappa}(\omega) \ldots \ldots . . .77$

4.2.3 Modelling of a nonuniform dielectric . . . . . . . . . 80

4.2.4 Effect of oxygen annealing on surface chemistry . . . . 81

4.2.5 Probing light penetration depth . . . . . . . . . . 83 
CONTENTS

xiii

4.3 Summary ... . . . . . . . . . . . . . . . . 84

4.4 Deduction of $c$-axis dielectric constant . . . . . . . . 85

5 Optical Conductivity of... 91

5.1 Pseudocubic $\mathrm{La}_{1-x} \mathrm{Ca}_{x} \mathrm{MnO}_{3}$. . . . . . . . . . . . . . . . . 93

$5.1 .1 \sigma_{1, \kappa \kappa}(\omega, T)$ for $\mathrm{La}_{0.9} \mathrm{Ca}_{0.1} \mathrm{MnO}_{3} \ldots \ldots \ldots . \ldots 9$

$5.1 .2 \quad \mathrm{La}_{0.735} \mathrm{Ca}_{0.265} \mathrm{MnO}_{3} \ldots \ldots \ldots . \ldots . \ldots 94$

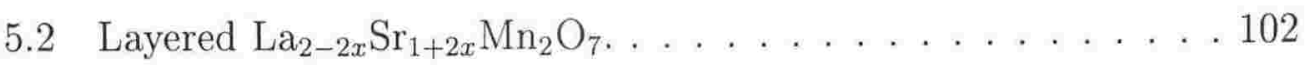

5.2.1 Magnetisation of $\mathrm{La}_{1.2} \mathrm{Sr}_{1.8} \mathrm{Mn}_{2} \mathrm{O}_{7} \ldots \ldots \ldots . . . . . .102$

$5.2 .2 \sigma_{1}(\omega, T)$ for $\mathrm{La}_{1.2} \mathrm{Sr}_{1.8} \mathrm{Mn}_{2} \mathrm{O}_{7} \ldots \ldots \ldots . . \ldots . . \ldots 104$

5.2 .3 Spectral weight changes . . . . . . . . . . 112

5.2.4 High-energy mode phonon analyses . . . . . . . . 118

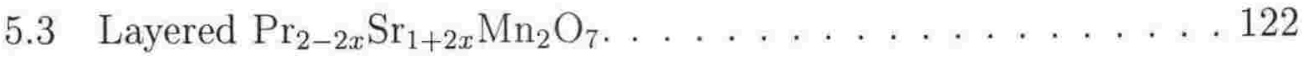

5.3 .1 Magnetisation . . . . . . . . . . . . 123

5.3.2 $\sigma_{1}$ by ellipsometry of $a b$ plane and $c$-axis $\mathrm{PrSr}_{2} \mathrm{Mn}_{2} \mathrm{O}_{7} \ldots \ldots \ldots \ldots . \ldots 124$

5.3.3 $\tilde{\sigma}_{\kappa \kappa}$ of $a b$ plane $\operatorname{PrSr}_{2} \mathrm{Mn}_{2} \mathrm{O}_{7}$ by normal incidence reflection . . . . . . . . . . . . . . . 129

5.3.4 Mid-IR band understood as a polaron . . . . . . . . . . 132

5.3.5 Phonon analysis . . . . . . . . . . . . . 134

5.3.6 Disorder and far-IR absorption . . . . . . . . 137

$\begin{array}{llr}6 & \text { In Conclusion } & 139\end{array}$

6.1 Further work . . . . . . . . . . . . . . . . . . . . 144

A Ellipsometric constant reduction $\quad 145$

A.1 Pre-reduction spectra . . . . . . . . . . . . . . . . 145

A.2 Uncertainties associated with reduction . . . . . . . . . . . 149

B Deduction of the $c$-axis response 151

C Normal Incidence vs Ellipsometry $\quad 155$ 


\section{List of Tables}

1 Energy conversions ................... vii

1.1 Phase transitions of manganite samples . . . . . . . . . . 14

3.1 Bomem DA8 spectrometer resources and their spectral range. 45

3.2 Spectral coverage of the Bruker $113 \mathrm{v}$ and Nicolet $400 \mathrm{spec}-$

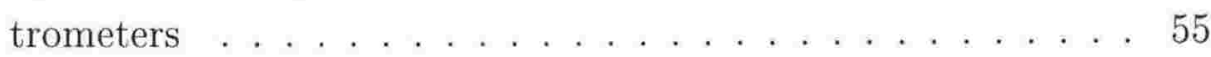

3.3 Manganite sample surface preparation treatments . . . . . . 59

5.1 Experimental parameters for manganite samples . . . . . . . . 92

5.2 Manganite phonon frequencies . . . . . . . . . . . . . . 111

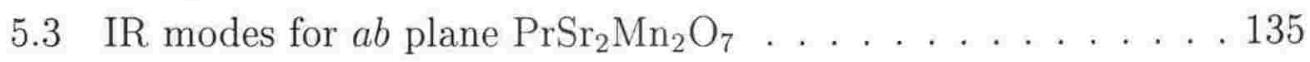




\section{List of Figures}

1.1 Magnetoresistivity of the $a b$ plane $\mathrm{La}_{1.2} \mathrm{Sr}_{1.8} \mathrm{Mn}_{2} \mathrm{O}_{7} \ldots \ldots$

1.2 Unit cell of the manganese oxide perovskite . . . . . . . . . 4

1.3 Structure of Ruddlesden-Popper series manganites . . . . . . . 5

1.4 Magnetic phase diagram of $\mathrm{La}_{1-x} \mathrm{Ca}_{x} \mathrm{MnO}_{3} \ldots \ldots . . . . .6$

1.5 Magnetic phase diagram of $(\mathrm{La} / \mathrm{Sr})_{3} \mathrm{Mn}_{2} \mathrm{O}_{7} \ldots \ldots 7$

1.6 Schematic diagram of the energy splitting $d$ level orbitals . . . 8

1.7 Schematic diagram of the Mn $d$ level orbitals . . . . . . . . . . 9

$1.8 \sigma_{1}(\omega)$ for a typical manganite . . . . . . . . . . . . . . . 11

1.9 Infrared active lattice vibrations . . . . . . . . . . . 12

2.1 Ellipsometry setup schematic . . . . . . . . . . . . 22

$2.2 a b$ plane and $c$-axis ellipsometry measurement . . . . . . . . 26

2.3 Schematic of the normal and oblique incidence measurements on the sample surface . . . . . . . . . . . . . . . . . . . 29

2.4 Schematic illustrating the connection between causality and

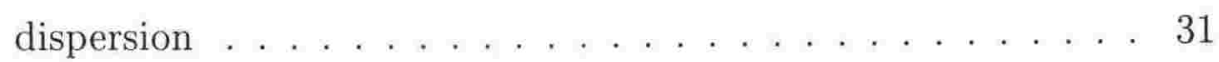

2.5 Lorentz dielectric constants and reflectance . . . . . . . . . 36

2.6 Drude dielectric constants and reflectance . . . . . . . . 38

3.1 Schematic of the Michelson interferometer and spectrometer . 43

3.2 Overlap of spectra measured using different spectrometer re-

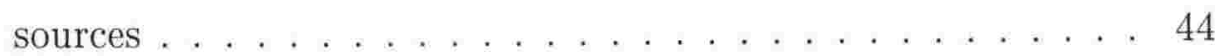

3.3 Optical bench used for normal incidence reflection . . . . . . 46

3.4 Schematic of the measurement and data reduction process for Normal Incidence Reflectivity . . . . . . . . . . . . . . 48

3.5 Reproducibility of the spectrometer and optical bench set-up . 50 
3.6 Schematic of the measurement and data reduction process for ellipsometry . . . . . . . . . . . . . . . . 5 53

3.7 Brookhaven ellipsometry setup schematic . . . . . . . . . 57

3.8 Brookhaven beamline spectral characteristic . . . . . . . . . 58

3.9 Laue diffraction pattern of $\mathrm{La}_{0.735} \mathrm{Ca}_{0.265} \mathrm{MnO}_{3} \ldots \ldots$. . . . . . 60

3.10 Annealing furnace profiles for $\mathrm{La}_{1-x} \mathrm{Ca}_{x} \mathrm{MnO}_{3}$. . . . . . . . . 62

3.11 Scanning Electron Microscope images of $\mathrm{La}_{0.9} \mathrm{Ca}_{0.1} \mathrm{MnO}_{3}$. . . 63

3.12 Magnetisation curves for $\mathrm{La}_{0.9} \mathrm{Ca}_{0.1} \mathrm{MnO}_{3}$. . . . . . . . . . . . 64

3.13 Magnetisation curves for $\mathrm{La}_{0.735} \mathrm{Ca}_{0.265} \mathrm{MnO}_{3} \ldots$. . . . . . . . 65

4.1 Reflectance extrapolations for annealed $\mathrm{La}_{0.9} \mathrm{Ca}_{0.1} \mathrm{MnO}_{3}$ at $295 \mathrm{~K} 69$

4.2 Reflectance of $\mathrm{La}_{0.9} \mathrm{Ca}_{0.1} \mathrm{MnO}_{3}$ before and after annealing . . . 71

$4.3 \sigma_{1}(\omega)$ for $\mathrm{La}_{0.9} \mathrm{Ca}_{0.1} \mathrm{MnO}_{3}$ before and after annealing . . . . . 72

4.4 Reflectance, $\sigma_{1, \kappa \kappa}(\omega)$, and $\epsilon_{1, \kappa \kappa}(\omega)$ spectra of $\mathrm{La}_{0.735} \mathrm{Ca}_{0.265} \mathrm{MnO}_{3}$ demonstrating the effect of different low energy $R(\omega)$ extrapolations .......................... 74

4.5 Reflectance and $\sigma_{1, \kappa \kappa}(\omega)$ spectra of $\mathrm{La}_{0.735} \mathrm{Ca}_{0.265} \mathrm{MnO}_{3}$ demonstrating the effect of different high energy $R(\omega)$ extrapolations 76

4.6 Reflectance and $\sigma_{\kappa \kappa}(\omega)$ spectra of $\mathrm{La}_{0.735} \mathrm{Ca}_{0.265} \mathrm{MnO}_{3}$ before and after annealing . . . . . . . . . . . . . 78

4.7 $R(\omega)$ and $\epsilon_{2}(\omega)$ for a model material with a 'damaged' surface layer . . . . . . . . . . . . . . . . . . . . 81

4.8 Secondary Ion Mass Spectrometry profiles of $\mathrm{La}_{0.735} \mathrm{Ca}_{0.265} \mathrm{MnO}_{3}$ before and after annealing . . . . . . . . . . . . . 82

4.9 Skindepth $\delta(\omega)$ of $\mathrm{La}_{0.735} \mathrm{Ca}_{0.265} \mathrm{MnO}_{3}$ at $295 \mathrm{~K}$ and $75 \mathrm{~K}$. . 83

$4.10 n$ and $k$ fits from ellipsometry and normal incidence measurements for the $\mathrm{PrSr}_{2} \mathrm{Mn}_{2} \mathrm{O}_{7}$ c-axis . . . . . . . . . 86

4.11 mid-IR $c$-axis optical constants for $\mathrm{PrSr}_{2} \mathrm{Mn}_{2} \mathrm{O}_{7}$ deduced from $a b$ plane measurements . . . . . . . . . . . . . . . 88

$5.1 \rho(T)$ of manganites in this study . . . . . . . . . . . . . . 93

$5.2 \sigma_{1, \kappa \kappa}(\omega, T)$ of unannealed $\mathrm{La}_{0.9} \mathrm{Ca}_{0.1} \mathrm{MnO}_{3} \ldots \ldots . \ldots 9$

5.3 Reflectance of annealed $\mathrm{La}_{0.735} \mathrm{Ca}_{0.265} \mathrm{MnO}_{3} \ldots \ldots$. . . . . . . . 95

$5.4 \sigma_{1, \kappa \kappa}(\omega)$ of annealed $\mathrm{La}_{0.735} \mathrm{Ca}_{0.265} \mathrm{MnO}_{3} \ldots \ldots . . . . . . .96$ 
$5.5 \mathrm{~N}_{\text {eff }}(\omega)$ for $\mathrm{La}_{0.735} \mathrm{Ca}_{0.265} \mathrm{MnO}_{3} \ldots \ldots \ldots \ldots \ldots$

$5.6 N_{\text {eff }}(0.5 \mathrm{eV})$ for $\mathrm{La}_{0.735} \mathrm{Ca}_{0.265} \mathrm{MnO}_{3} \ldots \ldots \ldots \ldots$

$5.7 \epsilon_{1, \kappa \kappa}(\omega)$ of annealed $\mathrm{La}_{0.735} \mathrm{Ca}_{0.265} \mathrm{MnO}_{3} \ldots \ldots \ldots \ldots$

5.8 Magnetisation for $a b$ plane and $c$-axis $\mathrm{La}_{1.2} \mathrm{Sr}_{1.8} \mathrm{Mn}_{2} \mathrm{O}_{7} \ldots \ldots$

5.9 Temperature-dependent mid-IR $\sigma_{1}(\omega)$ spectra by ellipsometry

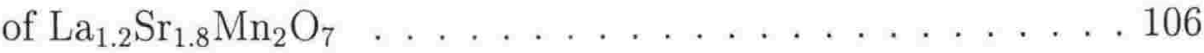

5.10 Temperature-dependent far-IR $\sigma_{1}(\omega)$ spectra by ellipsometry of $\mathrm{La}_{1.2} \mathrm{Sr}_{1.8} \mathrm{Mn}_{2} \mathrm{O}_{7} \ldots \ldots \ldots \ldots \ldots$

$5.11 \sigma_{1}(\omega, \mathrm{T})-\sigma_{1}(\omega, 250 \mathrm{~K})$ spectra for $\mathrm{La}_{1.2} \mathrm{Sr}_{1.8} \mathrm{Mn}_{2} \mathrm{O}_{7} \ldots \ldots \ldots 113$

5.12 Effective number of far-IR $c$-axis carriers in $\mathrm{La}_{1.2} \mathrm{Sr}_{1.8} \mathrm{Mn}_{2} \mathrm{O}_{7} .115$

$5.13 \mathrm{La}_{1.2} \mathrm{Sr}_{1.8} \mathrm{Mn}_{2} \mathrm{O}_{7}$ spectral weights and magnetisation . . . . 116

$5.14 \mathrm{La}_{1.2} \mathrm{Sr}_{1.8} \mathrm{Mn}_{2} \mathrm{O}_{7}$ spectral weights and temperature-dependent Double Exchange bandwidth . . . . . . . . . . . . . . 117

$5.15 \mathrm{La}_{1.2} \mathrm{Sr}_{1.8} \mathrm{Mn}_{2} \mathrm{O}_{7} a b$ plane phonon spectral weights . . . . . 119

$5.16 \mathrm{La}_{1.2} \mathrm{Sr}_{1.8} \mathrm{Mn}_{2} \mathrm{O}_{7} a b$ plane high-energy mode frequency shift $\ldots 120$

$5.17 \mathrm{La}_{1.2} \mathrm{Sr}_{1.8} \mathrm{Mn}_{2} \mathrm{O}_{7} c$-axis phonon parameters $\ldots \ldots \ldots \ldots . \ldots 121$

5.18 Magnetisation for $a b$ plane and $c$-axis $\operatorname{PrSr}_{2} \mathrm{Mn}_{2} \mathrm{O}_{7} \ldots \ldots 123$

5.19 Temperature-dependent $\sigma_{1}(\omega)$ far-IR spectra by ellipsometry

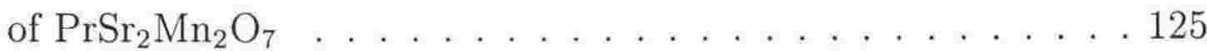

5.20 Temperature-dependent $\sigma_{1}(T, \omega)-\sigma_{1}(295 K, \omega)$ far-IR spectra of $a b$ plane $\mathrm{PrSr}_{2} \mathrm{Mn}_{2} \mathrm{O}_{7} \ldots \ldots \ldots \ldots \ldots$

5.21 Temperature-dependent mid-IR $R(\omega)$ spectra for $\operatorname{PrSr}_{2} \mathrm{Mn}_{2} \mathrm{O}_{7} 129$

5.22 Temperature-dependent mid-IR $\sigma_{1, \kappa \kappa}(\omega)$ spectra for $\operatorname{PrSr}_{2} \mathrm{Mn}_{2} \mathrm{O}_{7} 130$

5.23 Polaron absorption behaviour of the conductivity . . . . . . 133

A.1 Temperature-dependent far-IR unreduced (includes the interactive $c$-axis component) $\sigma_{1}(\omega)$ spectra for $\operatorname{PrSr}_{2} \mathrm{Mn}_{2} \mathrm{O}_{7}$ and $\mathrm{La}_{1.2} \mathrm{Sr}_{1.8} \mathrm{Mn}_{2} \mathrm{O}_{7} \ldots \ldots \ldots \ldots \ldots$

A.2 Far-IR reduced and unreduced $\sigma_{1}(\omega)$ and $\epsilon(\omega)$ spectra for $\mathrm{PrSr}_{2} \mathrm{Mn}_{2} \mathrm{O}_{7}$ at $10 \mathrm{~K} \ldots \ldots \ldots \ldots \ldots \ldots . \ldots \ldots$

A.3 Far-IR reduced and unreduced $\sigma_{1}(\omega)$ and $\epsilon(\omega)$ spectra for

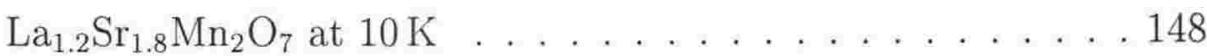


B.1 Model anisotropic materials illustrating extracted $c$-axis responses from $a b$ plane measurements . . . . . . . . . . . 152

C.1 Comparison between ellipsometry and normal incidence $R(\omega)$ and $\sigma_{1}(\omega)$ spectra for $\operatorname{PrSr}_{2} \mathrm{Mn}_{2} \mathrm{O}_{7} \ldots \ldots \ldots \ldots$ 


\section{Chapter 1}

\section{Colossal magnetoresistance manganites.}

For as long as matter, energy and the curiosity of mankind have coexisted, it can be said that the entity of Solid State Physics has existed also, lying dormant and waiting to be given form. As the nourishment of mathematical description and scientific discovery have been supplied, so the descriptions of matter and its physical behaviour have progressed. The efforts of the 20th century, in particular, have brought us to an advanced understanding of many of the peculiar properties exhibited by solids. This understanding leans heavily upon the quantum theory developed in the 1920's, which makes a very successful distinction between the two most antipodal materials in our experience, metals and insulators.

The band theory developed in order to make this distinction, in the first instance, was a consequence of the overlapping atomic energy levels, and thus depended entirely on the configuration of the crystal ions and their electrons, with no regard to electron interactions. Metals were thus defined to be those materials for which a band was partly filled, insulators, materials with full or empty bands, and semiconductors, those materials with a small band gap, which allowed conduction if the thermal energy of the electrons was greater than the gap energy. Although this theory worked remarkably well, its successes did not extend to the transition metal oxides, which, as observed in the late 1930's, were insulators despite having partially filled bands. It was at this time that the importance of electron-electron correlations was 
first suggested, and the Mott insulator proposed, written within the realms of band theory, to account for insulating behaviour in materials traditionally expected to be metallic.

However, although band theory accommodates some aspects of correlatedelectron systems, in recent times scientists have begun to tackle materials in which these weakly-interacting electron approximations fail to adequately describe their physical properties, materials in which the electrons can not be assumed to be only weakly interactive. This is the domain of the so-called strongly correlated electron systems ${ }^{1}$.

There are many high profile examples of materials which cannot be well characterised by the conventional independent electron approaches. These include the High Temperature Superconductors[2], conducting polymers[3], various metal oxides[1], and the subjects of this study, the manganites (manganese oxides) $[1,4]^{2}$. Each of these different types of material manifest very unusual physical properties: zero resistivity at temperatures much higher than expected before 1986[5], doped plastics which are metallic, and colossal magnetoresistance, to name a few examples.

Early work on the transition metal oxides appreciated the intriguing properties of the manganites in particular[ $[7,8,9,10,11]$. However, it wasn't until quite recently that research efforts were directed back to the field, the impetus for which was the observation of colossal magnetoresistance in the manganite crystals $[12,13,14,15]$. The phenomenon of colossal magnetoresistance has been hallowed as second only to superconductivity on the technology industry wish-list[16]. The excitement generated in the scientific community by materials which display such a huge electronic response to a magnetic field, anticipates that the manganites could be used as sensors, with a particular eye to such device applications as magnetic read heads. This colossal magnetoresistance property is well illustrated by the features displayed in Fig. 1.1, for one member, the layered manganite, $\mathrm{La}_{1.2} \mathrm{Sr}_{1.8} \mathrm{Mn}_{2} \mathrm{O}_{7}$. The resistivity in a magnetic field strength of 0 Tesla, illustrated in panel (a) from Moritomo and coworkers[6], first of all displays a sharp transition as the temperature

\footnotetext{
${ }^{1}$ Ref. [1] and references therein give a thorough review of this history.

${ }^{2}$ The citations are for selected review papers which are available for each material.
} 


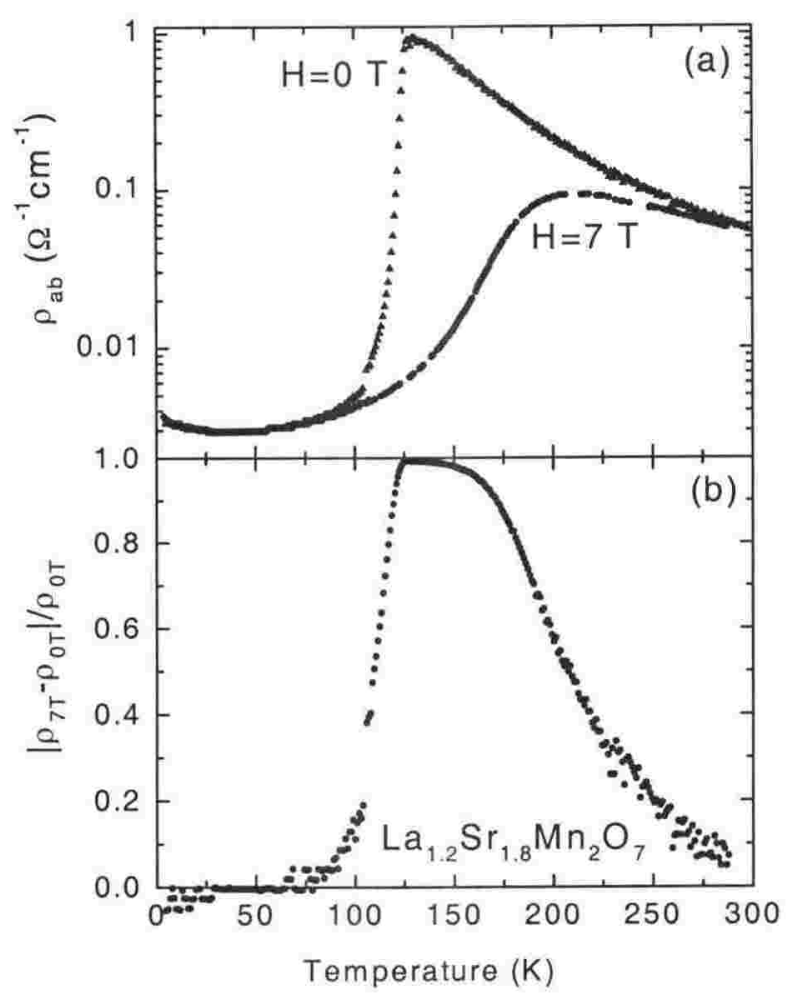

Figure 1.1: (a) $a b$ plane resistivity of $\mathrm{La}_{1.2} \mathrm{Sr}_{1.8} \mathrm{Mn}_{2} \mathrm{O}_{7}$ in zero magnetic field (triangles), and with $\mathrm{H}=7$ Tesla applied parallel to the $a b$ plane (circles), from ref. [6] and (b) the absolute value of the magnetoresistance.

is decreased through $125 \mathrm{~K}$. It can also be seen in Fig. 1.1(a) that upon the application of an external field, here 7 Tesla, the electrical resistivity of the material decreases by a significant amount and across a broad temperature range. The (negative) magnetoresistance, or change in resistivity with magnetic field is defined as

$$
\frac{\rho(H)-\rho(H=0)}{\rho(H=0)},
$$

where $\rho$ is the resistivity and $H$ the applied magnetic field. Calculating and displaying this in Fig. 1.1(b), we observe that the greatest magnetoresistance occurs near the insulator-metal transition temperature at $125 \mathrm{~K}$, with 
a value of $-99.3 \%$. The name colossal, to describe the astonishing magnitude of the effect, is a nod to the fact that a magnetoresistance of this magnitude has not even been observed in the so-called giant magnetoresistance materials. These consist of magnetic multilayers and granular materials in which the magnetoresistance reaches a maximum of the order of 80\%[17]. Worth remarking also is that the colossal magnetoresistance effect is observed in single crystals, and must be thus intrinsic to the manganite structure.

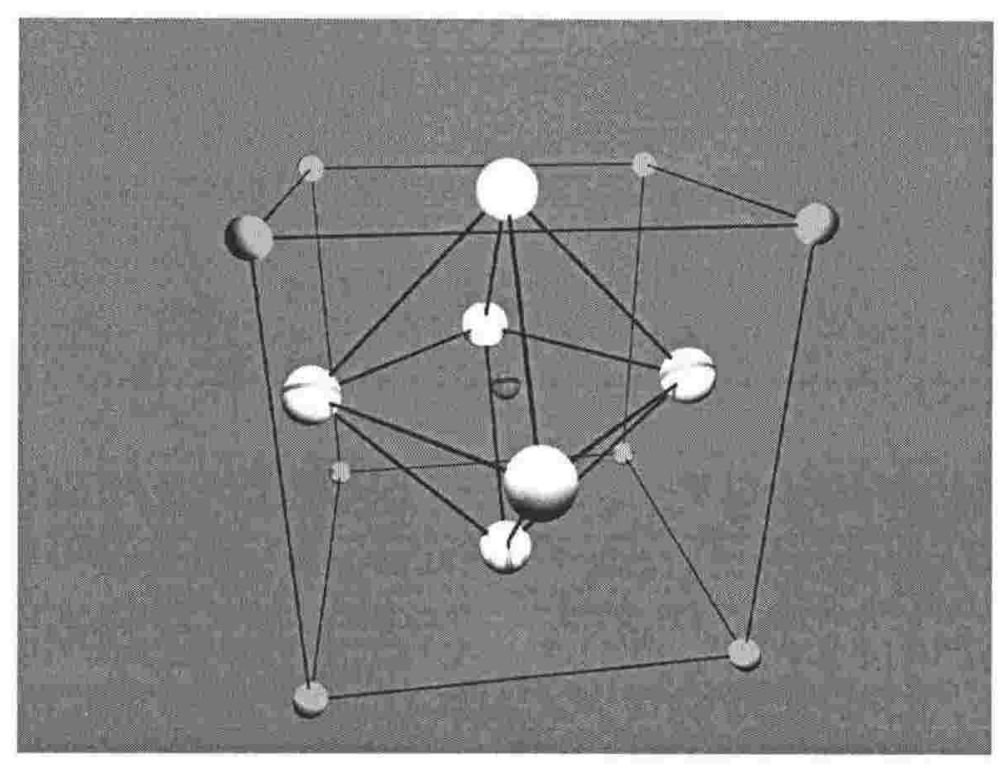

Figure 1.2: Schematic diagram of the unit cell of the manganese oxide perovskite, $(\mathrm{RE} / \mathrm{AE}) \mathrm{MnO}_{3}$. The corner positions are occupied by the $\mathrm{RE} / \mathrm{AE}$ ions, at the centre is the manganese ion, and this is surrounded by an "octahedral cage" of oxygen ions.

That structure is pictured in Fig. 1.2 and Fig. 1.3. The manganites belong to the Ruddlesden-Popper series with general formula $(\mathrm{RE} / \mathrm{AE})_{n+1} \mathrm{Mn}_{n} \mathrm{O}_{2 n+1}(n=1,2, \ldots \infty)$, where $\mathrm{RE}$ is a rare earth ion, $\mathrm{AE}$ an alkaline earth ion and where $n$ denotes the number of stacked $\mathrm{MnO}_{6}$ octahedral layers. The $\mathrm{MnO}_{6}$ octahedron is the basic building block of the different members of the Ruddlesden-Popper series, and it is pictured in its undistorted form in Fig. 1.2. In this diagram, the manganese ion sits in the middle of an octahedron of 6 oxygen ions, which is positioned in a cube, the corners of which comprise the rare earth or alkaline earth ions. 
The $\mathrm{MnO}_{6}$ building block constructs different manganite compounds as displayed in Fig. 1.3. The $n=\infty$ series on the far right of the diagram are the pseudo-cubic manganites with perovskite structure, and were the first to receive experimental attention[7]. The $n=1,2$ layered materials have more recently come in for exploration[6]. This study comprises samples from the $n=2$ and $n=\infty$ groups. As can be seen from the diagram the crystal structure of the $n=2$ family is uniaxially anisotropic, resulting in highly anisotropic DC resistivities (see Fig. 5.1 of Chapter 5).

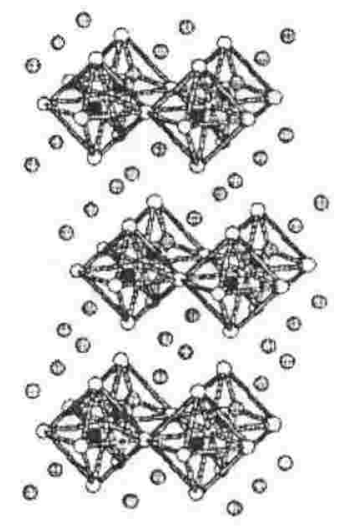

$n=1$

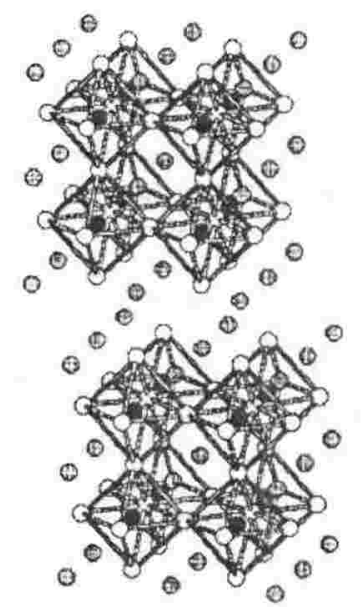

$n=2$

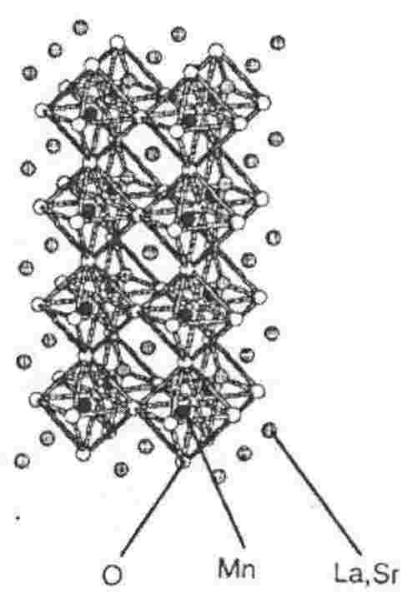

$n=\infty$

Figure 1.3: Stucture of the Ruddlesden-Popper series of manganites for $n=$ $1, n=2$ and $n=\infty$ from ref. [6].

The colossal magnetoresistance effect is not the only signature of strongly interacting electrons in the manganites. Other evidence comes in the form of the magnetic phase diagrams of the manganites, two of which have been included to motivate the samples studied in this work. The first can be seen in Fig. 1.4 for $\mathrm{La}_{1-x} \mathrm{Ca}_{x} \mathrm{MnO}_{3}$, one of the isotropic $n=\infty$ materials. The acronyms in the figure are translated in its legend ${ }^{3}$.

It can be seen that at room temperature, or $300 \mathrm{~K}$, the material is a paramagnetic insulator regardless of the hole substitution level, $x$. When the temperature is decreased, the unsubstituted material undergoes a transition

\footnotetext{
${ }^{3}$ To remind, $T_{N}$ and $T_{c}$ are respectively the Néel temperature, at which antiferromagnetic order sets in, and the Curie temperature, at which ferromagnetic order sets in.
} 


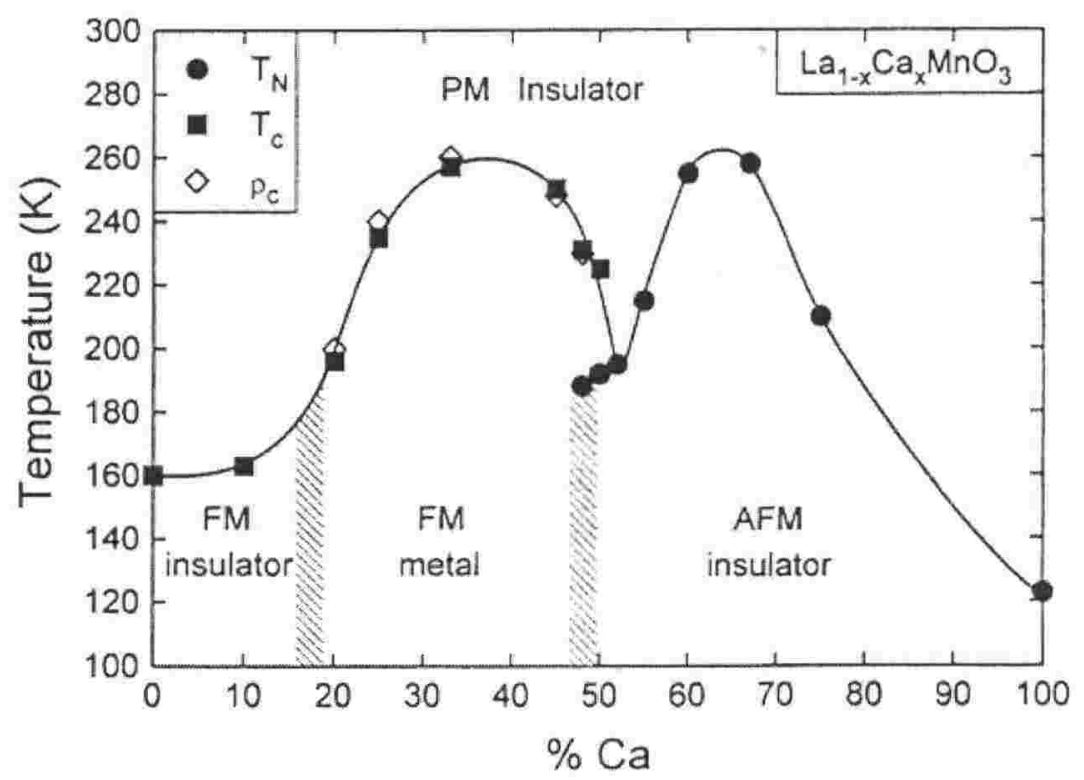

Figure 1.4: Magnetic phase diagram of $\mathrm{La}_{1-x} \mathrm{Ca}_{x} \mathrm{MnO}_{3}$ for $x=0.0-1.0$ from ref. [18]. The circle, square and diamond markers refer to whether the magnetic $\left(T_{N}, T_{c}\right)$ or resistive $\left(\rho_{c}\right)$ transitions, respectively, were used to define the transition temperature. The acronyms for the different phases are as follows: FM, ferromagnetic, AFM, antiferromagnetic, and PM, paramagnetic.

to a ferromagnetic insulator. As calcium $(\mathrm{Ca})$ is substituted onto the rare earth site, the material at low temperature undergoes a number of electronic and magnetic transitions, becoming metallic for $0.19<x<0.5$, and an insulating antiferromagnet for $x>0.5$.

As the structure of the manganite grows more complicated, now considering the anisotropic $n=2$ material, the phase diagram also grows in complexity as seen in Fig. 1.5 for $\mathrm{La}_{2-2 x} \mathrm{Sr}_{1+2 x} \mathrm{Mn}_{2} \mathrm{O}_{7}$. The acronyms in the Figure are again translated in the legend.

Like $\mathrm{La}_{1-x} \mathrm{Ca}_{x} \mathrm{MnO}_{3}$, the material at room temperature is a paramagnetic insulator regardless of substitution level. However, there is, at $300 \mathrm{~K}$, a structural change from tetragonal to orthorhombic with the crossing from $x<0.74$ to $x>0.75$. The low temperature ground state of $x=0.3$ is seen to be an antiferromagnetic metal. As the substitution level $x$ is varied the ground state $(T=0)$ properties vary across an enormous range of different 


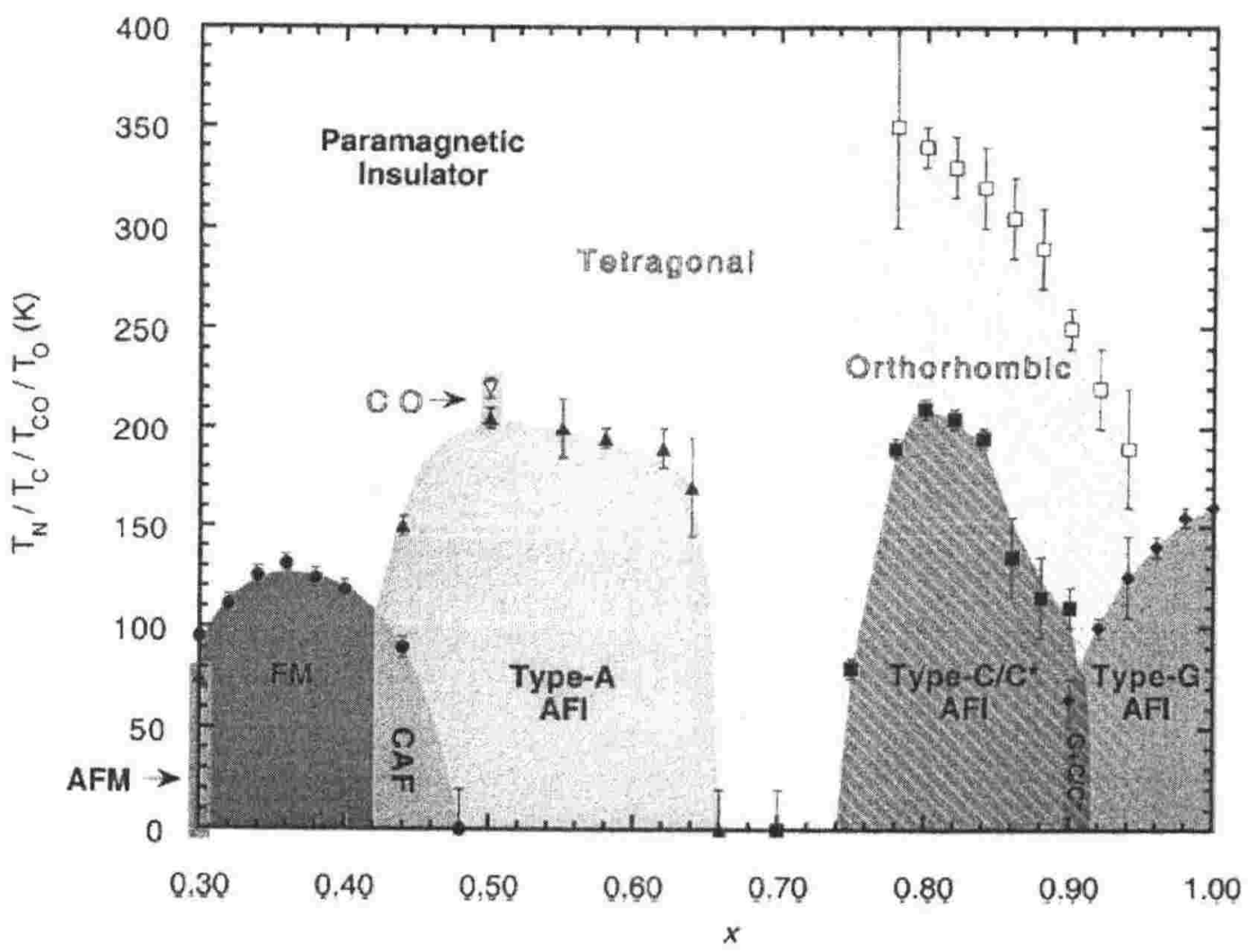

Figure 1.5: Magnetic phase diagram of $\mathrm{La}_{2-2 x} / \mathrm{Sr}_{1+2 x} \mathrm{Mn}_{2} \mathrm{O}_{7}$ for $x=0.3-1$ from ref. [19]. The various acronyms translate as follows: FM ferromagnetic metal, AFI, antiferromagnetic insulator, CAF canted antiferromagnetic, CO charge ordered state, and PM, paramagnetic.

magnetic orderings (the details of the exact types of AF order are not important here), passing through metal-insulator transitions in the region of $0.3<x<0.48$. The phase diagrams are very rich for all manganite compounds. That such a wide range of physical properties can be 'dialed up' by varying only one or two parameters in a compound, for example temperature, substitute ion and substitution level, lends this field wide ranging opportunities for the study of electronic correlations.

The key to understanding the diverse behaviour observed in the magnetic phase diagrams, and the explanation of the colossal magnetoresistance behaviour, lies in acquiring a better understanding of the interplay of electron charge, spin, orbital and structural characteristics driving these processes. 
We now discuss the electron dynamics in these materials.

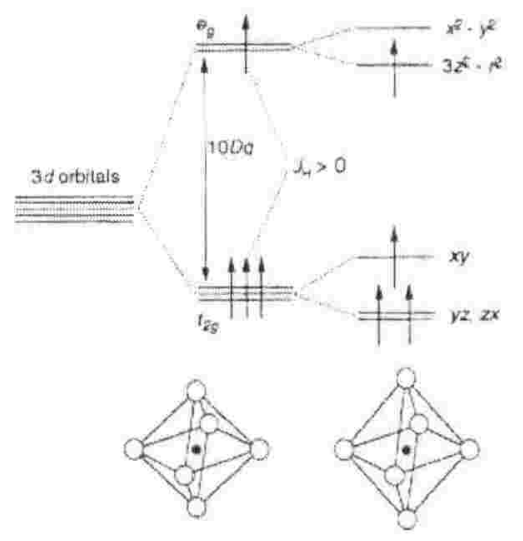

Figure 1.6: Energy level diagram of the crystal field splitting of the $d$ orbitals, from ref. [4].

The splitting of the degenerate $5 d$ orbitals is illustrated in the energy level diagram of Fig. 1.6. For the $\mathrm{RE}_{n+1} \mathrm{Mn}_{n} \mathrm{O}_{3 n+1}$, or unsubstituted material, the $\mathrm{Mn}^{3+}$ ions have 4 outer shell $(d)$ electrons. The first perturbation is that of the crystal field provided by the six surrounding $\mathrm{O}^{2-}$ ions. This splits the five degenerate orbitals into a triply degenerate $t_{2 g}$ state and a doubly degenerate $e_{g}$ state. Provided the thermal energy of the system is not too great, three of the electrons obey the Pauli exclusion principle and Hunds rules, lining up with identical spins on $t_{2 g}$ sites for a total of $S=3 / 2$. The fourth electron takes the $e_{g}$ state. Because it is strongly coupled to the core spins $\left(3 t_{2 g}\right)$, a fact that plays a significant role in the conduction properties of the manganites, it aligns itself with the spin of the $t_{2 g}$ electrons for a total spin of $S=2$. The energy of the system is lowered further by a Jahn-Teller distortion of the lattice, illustrated by the deformation of the $\mathrm{MnO}_{6}$ octahedron in Fig. 1.6. This renders the $3 z^{2}-r^{2}$ orbital configuration of the $e_{g}$ electron energetically favourable, while the $x^{2}-y^{2}$ orbital state sits at an energy $\Delta_{J T}$ above this. Note that the orbital degeneracy is also split in the $t_{2 g}$ levels.

The orbital shapes are illustrated in Fig. 1.7, which shows the $3 t_{2 g}$ levels, with $x y, y z, x z$ symmetry and the two $e_{g}$ levels with $3 z^{2}-r^{2}$ or $x^{2}-y^{2}$ symmetry. We can see in an eye-balling way, by comparing the distorted 


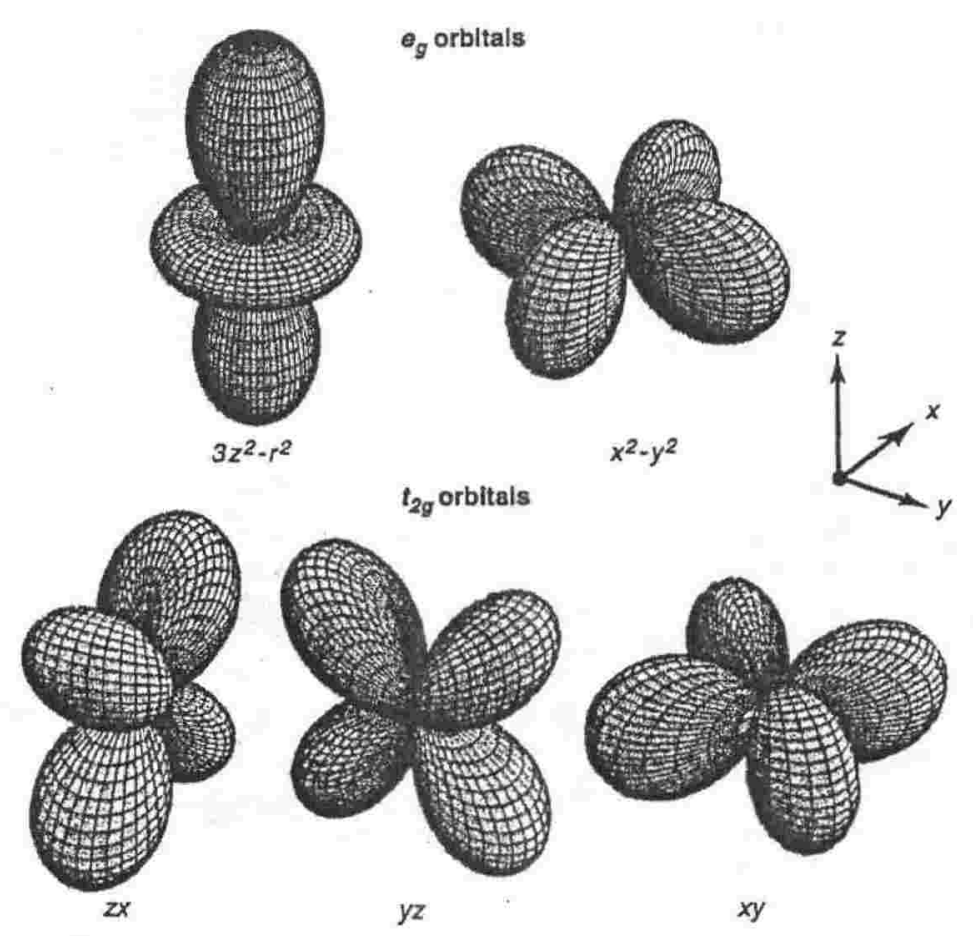

Figure 1.7: Schematic diagram of the Mn $d$ level orbitals from ref. [20]. For $\mathrm{Mn}^{3+}$ there are $3 t_{2 g}$ electrons in $x y, y z, x z$ orbitals, with the fourth electron occupying one of the two possible $3 z^{2}-r^{2}$ or $x^{2}-y^{2}$ orbital states.

octahedron of Fig. 1.6 with the two $e_{g}$ orbital shapes, that the elongated $3 z^{2}-r^{2}$ orbital would have less coulombic repulsion, and thus indeed be the energetically favourable state for the fourth electron.

The above discussion is for an unsubstituted manganite. If we now introduce holes into the material by replacing $\mathrm{RE}^{3+}$ ions with $\mathrm{AE}^{2+}$ ions in the parent compound, the physics gets more interesting. In the first instance, the substitution of a foreign ion with a different ionic radius produces internal pressure and changes to the structure. As we have just remarked, changes in structure can lead to changes in electron orbital configurations, and therefore shifts in the energy level diagrams. Also, the holes introduce intermediary sites via which the itinerant electrons, that is, those in the $e_{g}$ states, can hop from site to site. This process can be switched on or off by the degree of magnetic order in the system. If the core spins between neighbouring $\mathrm{Mn}^{3+}$ 
and $\mathrm{Mn}^{4+}$ ions either side of an $\mathrm{O}^{2-}$ are aligned, the electron on the $\mathrm{Mn}^{3+}$ site may hop to the intermediate $\mathrm{O}^{2-}$ ion, which simultaneously passes one of its electrons to the $\mathrm{Mn}^{4+}$ site. This is the Double-Exchange process [7, 8]. In the paramagnetic state this conduction process is blocked. This is because the energy required for the $e_{g}$ electron to hop to a vacant site where the core spins are aligned antiparallel to it is prohibitive. Double exchange explains why metallic behaviour accompanies the onset of ferromagnetic order, as seen in the phase diagrams of Fig. 1.4 and Fig. 1.5.

At low hole concentrations (see Fig. 1.4 and Fig. 1.5), the density of open sites is not great enough for long range hopping and so the material remains insulating, even when all the spins are ferromagnetically aligned at low temperature. Further, at $x=0.5$ the manganites exhibit a tendency to order so that $\mathrm{Mn}^{3+}$ and $\mathrm{Mn}^{4+}$ sites alternate. In this scenario a sharp decrease in the long-range hopping conduction is observed. This is the phenomenon of charge order.

This brief introduction to the characteristics of the manganites has allowed us to survey the important degrees of freedom at the electronic level of the manganites. We see firstly the importance of the electron-lattice interaction in determining the energies of the system. Secondly, we have considered the driver of electron-electron interactions. These manifest themselves in the Hunds rule coupling and in the orbital cloud interactions. The real progress toward pinpointing behaviours in the colossal magnetoresistance manganites will be in isolating the important energies in the systems. This may be achieved by measuring the energy-dependent (optical) conductivity of the materials. When light is incident on a surface the material interacts with the radiation in a way that betrays these electronic and molecular characteristics. The percentage of light reflected from a surface as a function of frequency yields information on the dielectric constant or response function of that material. The way in which a material responds to incident light depends on the electronic interaction. The optical conductivity measurements therefore may well be the key to unravelling the different electronic and lattice contributions to the colossal negative magnetoresistance observed in the manganites. In the next section we background some of the progress that 
has been made already in this area, the uncharted areas of the field and how this project contributes to the mapping of the manganites.

\subsection{Conductivity of manganites.}

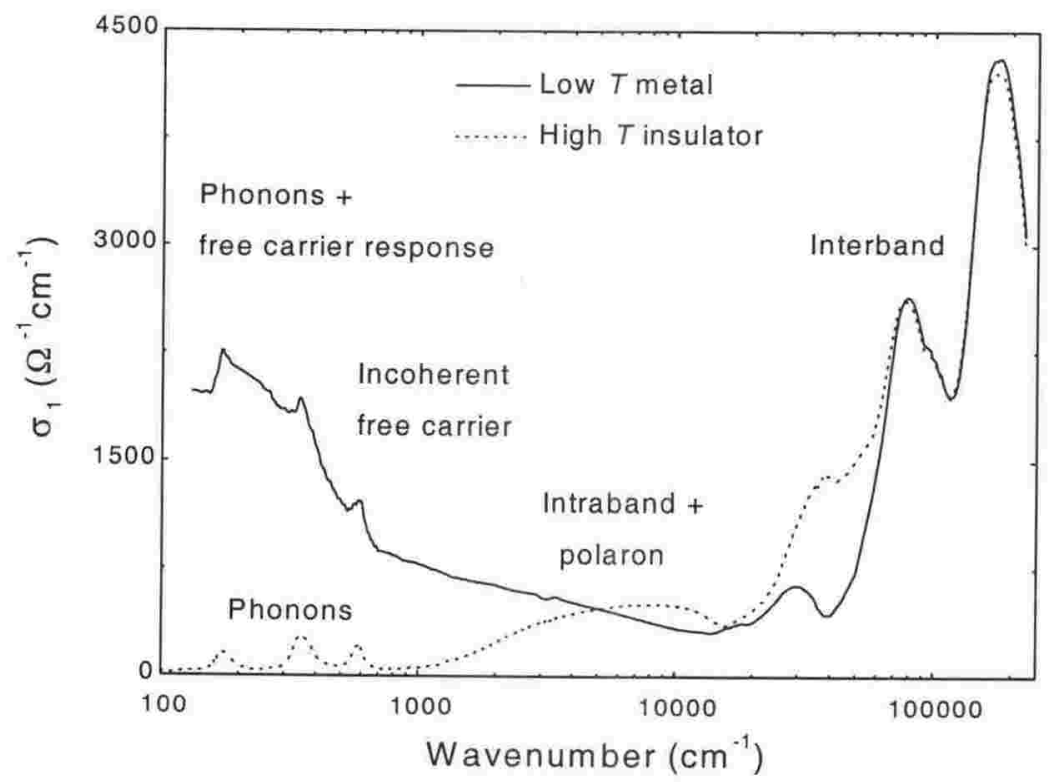

Figure 1.8: $\sigma_{1}(\omega)$ for a typical manganite in both of its insulating (dotted line) and metallic (solid line) phases. The text portions in the figure, in the energy regions where they appear, are a guide to the types of transition taking place there.

Although their properties are diverse, the manganites display many common features in spectra of $\sigma_{1}(\omega)$, or the real part of the optical conductivity. Displayed in Fig. 1.8 are two spectra representing the states that exhibit the most contrast in conductivity measurements; the metallic (solid line) and insulating (dotted line) phases ${ }^{4}$. We describe now the transitions that

\footnotetext{
${ }^{4}$ Although even the distinctions between metals and insulators are blurred by these complex materials, for the purposes of this thesis we 'define' an insulator to have $\rho(T) \rightarrow \infty$ as $T \rightarrow 0$. We describe a material which has $\rho(T)$ decreasing with temperature, as being metallic.
} 
contribute to the different energy regions.

Starting from the low energy end, we observe in both the metallic and insulating phases, three distinct peaks corresponding to lattice vibrations or phonons. The exact vibrations contributing to each of these energies is displayed in Fig. 1.9. As proposed by Kim and coworkers[22], the vibration with

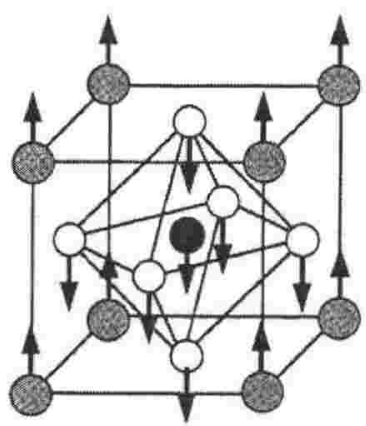

external mode

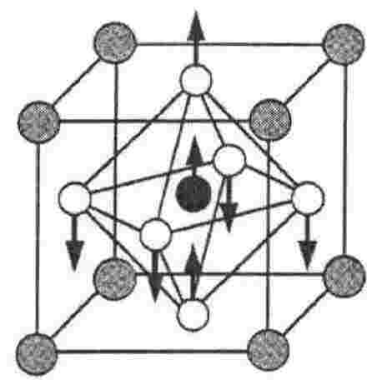

bending mode

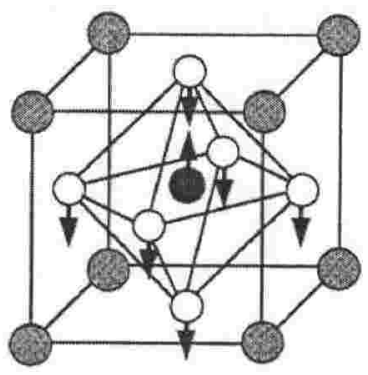

stretching mode

Figure 1.9: Proposed vibrations of the $\mathrm{MnO}_{6}$ octahedral cage that contribute to the observed infrared active absorptions, reproduced from ref. [21].

the greatest effective mass, that of the rare or alkaline earth ions against the octahedral cage, is responsible for the lowest energy phonon. The "bending" phonon is the next highest in energy, and is contributed to by the bending of the O-Mn-O articulated bond in the octahedron. The highest energy mode is caused by the stretching vibration of the Mn-O bonds. However, as readily appreciated from the diagram in Fig. 1.9, none of these IR vibrations is independent of the others. The "stretching" mode for instance, also involves a bending of the O-Mn-O bond. Because of this it is very difficult to assign IR phonon excitations independently.

At high energy now, the temperature-independent interband transition between $60000 \mathrm{~cm}^{-1}$ and $80000 \mathrm{~cm}^{-1}$ has been assigned to the rare earth or alkaline earth $d$ electron transitions, and the highest energy peak to $\mathrm{Mn}$ $4 s / 4 p$ transitions $[23,24]$.

Now we turn to the less well-characterised transitions. In the metallic phase, a "free" carrier response (as will be detailed in the next chapter) of coherently moving charges results in the broad feature which peaks at zero 
frequency, depicted by the solid line of Fig. 1.8. This is a feature which, when modelled by the conventional metallic, or Drude, model, gives inconsistencies with the expected Double Exchange behaviour[25, 26]. Moving along, the energy region between $1000 \mathrm{~cm}^{-1}$ and $20000 \mathrm{~cm}^{-1}$ is poorly modelled by just one electron transition, a fitting which constitutes a first approach to understanding the energy region. For example, a study which initially treated this band as due to an $e_{g} \rightarrow e_{g}$ transition between Jahn Teller split levels on a $\mathrm{Mn}^{3+}$ atom[23, 27], was revised to include the $e_{g} \rightarrow e_{g}$ transition from a $\mathrm{Mn}^{3+}$ to a $\mathrm{Mn}^{4+}$ site[28]. Moreover, there are almost as many interpretations as to the origin of this peak as there are studies. For instance, it is also held that this band is due to an $e_{g} \rightarrow e_{g}$ transition between $\mathrm{Mn}^{3+}$ atoms[29], to small polarons[30], to transitions related to Jahn-Teller clusters[31] and to a combination of small polaron and unnamed transition[32]. Many studies simply err on the side of caution, and discuss the peak with no attempt at assignment[26, 33]. Obviously there is much work to be done in resolving this debate. Lastly, some controversy exists as to the origin of the $\approx 300000$ $\mathrm{cm}^{-1}$ band also, and whether this constitutes a transition from the oxygen ion involving the $t_{2 g}[24,25,34]$ or $e_{g}$ electron[23, 29, 32].

To address the discrepancies between the data and simple models of it, electron-lattice or polaron interactions are usually brought to bear. One of the longstanding issues in this area however, is how appropriate are the different models of the polaron behaviour to conductivity data? Is there another adequate description for the de-enhancement of the free carrier conductivity, localisation due to disorder for instance? Is a more circumspect approach to modelling appropriate, given the dearth of complete and even reliable $\sigma_{1}(\omega)$ spectra for some members of the manganite family?

The purpose of this thesis, then, was to measure the optical conductivity of various samples of manganite in the energy region of contention, and to compare these spectra against existing models for manganite behaviour. Two methods were used to measure optical constants, ellipsometry and normal incidence reflectivity, both of which enable measurements in the infrared region. Ellipsometry measures real and imaginary parts of the dielectric constant, enabling us to examine and analyse the absorptive features in the spectra. Far 


\begin{tabular}{|c|c|c|c|c|c|}
\hline Sample & $x$ & $T_{M I}$ & $T_{c}$ & $T_{N}$ & $T=10 \mathrm{~K}$ properties \\
\hline \multirow[t]{2}{*}{$\mathrm{La}_{1-x} \mathrm{Ca}_{x} \mathrm{MnO}_{3}$} & 0.1 & - & $140 \mathrm{~K}$ & - & FM Insulator \\
\hline & 0.265 & $205 \mathrm{~K}$ & $205 \mathrm{~K}$ & - & FM Metal \\
\hline \multirow{2}{*}{$\begin{array}{r}\mathrm{La}_{2-2 x} \mathrm{Sr}_{1+2 x} \mathrm{Mn}_{2} \mathrm{O}_{7} \\
a b \text { plane } \\
c \text {-axis } \\
\end{array}$} & \multirow[t]{2}{*}{0.4} & $125 \mathrm{~K}$ & $125 \mathrm{~K}$ & - & FM Metal \\
\hline & & - & $125 \mathrm{~K}$ & - & FM Insulator \\
\hline \multirow{2}{*}{$\begin{array}{r}\mathrm{Pr}_{2-2 x} \mathrm{Sr}_{1+2 x} \mathrm{Mn}_{2} \mathrm{O}_{7} \\
a b \text { plane } \\
c \text {-axis }\end{array}$} & \multirow[t]{2}{*}{0.5} & - & - & $130 \mathrm{~K}$ & AFM Insulator \\
\hline & & - & - & - & PM Insulator \\
\hline
\end{tabular}

Table 1.1: Magnetic and electronic phase transitions of each sample measured, where $\mathrm{T}_{M I}$ denotes the insulator-metal transition temperature, $\mathrm{T}_{c}$, the Curie temperature, below which the material becomes ferromagnetic (FM), and $\mathrm{T}_{N}$, the Néel temperature, below which the material orders antiferromagnetically (AFM).

infrared ellipsometry in particular, is a recently developed technique[35], and as such has not been used to measure the properties of manganites prior to this study. Normal incidence reflection requires the measurement of the reflection intensity at a large range of energies, in order to extract the real and imaginary parts of the dielectric constant. Careful measurement and analysis of the data has been performed for four different manganite single crystals. Single crystals are indispensable for optical measurements, as spectral features in polycrystalline material can be broadened artificially by crystalline disorder in the sample.

Table 1.1 shows a summary of the materials that were studied in this thesis, along with the temperatures at which phase transitions occur in the samples measured. A variety of materials are represented, and in turn their properties are diverse. They, in fact, encompass many of the different phases observed in the manganites, from paramagnetic insulator to ferromagnetic metal to antiferromagnetic insulator.

This study presents measurements of the optical conductivity of a high photometric accuracy, for each of the four samples described in table 1.1. The results have been discussed against a backdrop of polaron and localisation theories which are applied to the manganites, and comments are made herein 
1.1. CONDUCTIVITY OF MANGANITES.

as to the applicability of these models. 


\section{Chapter 2}

\section{Background Theory.}

This chapter aims to set the scene for the theory that underpins the experimental measurement of a material's response to optical stimuli. Maxwell's equations will serve as the starting point for discussion of how the electric field, a detectable quantity, behaves at an interface. The second section will deal with how the polarisation changes in light that is obliquely incident on a material surface are used to find the dielectric constant in the technique of ellipsometry. The next section will discuss the different approach necessary in the use of normal incidence reflectivity to find the dielectric constant. Lastly, oscillator models will be discussed, which give us the interpretive framework within which to characterise results of our measurement of the dielectric constants.

\subsection{Light and its interaction with a medium.}

\subsubsection{Maxwell's equations.}

In an isotropic, energy absorbing medium with no net charge or current density, Maxwell's equations read

$$
\begin{gathered}
\nabla \cdot \mathbf{E}=0 \\
\nabla \times \mathbf{E}=-\frac{\mu}{c} \frac{\partial \mathbf{H}}{\partial t} \\
\nabla \cdot \mathbf{H}=0
\end{gathered}
$$




$$
\nabla \times \mathbf{H}=\frac{\epsilon}{c} \frac{\partial \mathbf{E}}{\partial t}+\frac{4 \pi \sigma}{c} \mathbf{E},
$$

where $\epsilon$ is the dielectric constant, $\mu$ the magnetic permeability and $\sigma$ the conductivity of the medium the light is propagating through.

The wave equation for the electric field, deduced by using equations 2.2 and 2.4 , a vector identity and equation 2.1 is

$$
\nabla^{2} \mathbf{E}=\frac{\epsilon \mu}{c^{2}} \frac{\partial^{2} \mathbf{E}}{\partial t^{2}}+\frac{4 \pi \sigma \mu}{c^{2}} \frac{\partial \mathbf{E}}{\partial t}
$$

which has a solution of the form

$$
\mathbf{E}(\mathbf{r}, t)=\mathbf{E}_{0} e^{(\tilde{\mathbf{q}} \cdot \mathbf{R}-i \omega t)}
$$

where $q$ is the wavevector

$$
\tilde{q}^{2}=\mu \frac{\omega^{2}}{c^{2}}\left(\epsilon+i \frac{4 \pi \sigma}{\omega}\right) .
$$

We define a complex refractive index $\tilde{n}=n+i k$, where $n$ is known as the refractive index and $k$ the extinction coefficient, and let $\tilde{q}=(\omega / c) \tilde{n}$. With this definition and equation 2.7, we get that $\epsilon=\left(n^{2}-k^{2}\right) / \mu$ and $4 \pi \sigma / \omega=2 n k / \mu$, where $\sigma$ is taken as the real part of the conductivity. We take the magnetic permeability to be $\mu=1$. Defining a complex dielectric function as $\tilde{\epsilon}=\epsilon_{1}+i \epsilon_{2}=\tilde{n}^{2}$, we gain the expressions $\epsilon_{1}=n^{2}-k^{2}$ and $\epsilon_{2}=2 n k$. Equipped with these descriptions, we may now more closely examine light and its interaction with a medium, and how we may extract the dielectric constant of the medium using light as our probe.

\subsubsection{Behaviour of $\mathrm{E}$ at an interface.}

Let us now consider the behaviour of a light wave at the boundary between two media with different dielectric constants. Let us give the wave an electric field $\mathbf{E}(\mathbf{r}, t)=\mathbf{E}_{0} e^{(\tilde{\mathbf{q}} \cdot \mathbf{r}-i \omega t)}$ and corresponding magnetic field $\mathbf{H}(\mathbf{r}, t)=$ $\mathbf{H}_{\mathbf{0}} e^{(\tilde{\mathbf{q}} \cdot \mathbf{r}-i \omega t)}$ and consider the beam having an incident angle of $\theta$ to the boundary surface normal. The wave is incident from a medium with refractive index $\tilde{n}_{0}$, and is reflected from a medium with refractive index $\tilde{n}$. In this project our boundary materials are always air and the sample in question, so 
we need only consider the case where the medium of incidence has $\tilde{n}_{0}=1$, the refractive index of a vacuum. In order to apply boundary conditions at the interfacial surface, we consider the components of light with electric field parallel and perpendicular to the plane of incidence separately. These are labelled $E_{\|}$and $E_{\perp}$ respectively. We choose subscripts $r$ and $t$ to denote the reflected and transmitted components of the electric and magnetic fields, and describe the incident components by omitting the subscript altogether.

Components of the electric and magnetic fields orthogonal to the incident plane must be continuous across the interfacial surface, so (recalling the phase change upon reflection of orthogonally polarised fields) this immediately gives us the boundary conditions

$$
E_{\perp}+E_{\perp r}=E_{\perp t}
$$

and

$$
H_{\perp}+H_{\perp r}=H_{\perp t}
$$

which can be rewritten in terms of $\mathrm{E}$ as

$$
E_{\|}+E_{\| r}=\tilde{n} E_{\| t}
$$

The expressions for the electric and magnetic field components parallel to the incident plane are

$$
E_{\|} \cos \theta-E_{\| r} \cos \theta=E_{|| t} \cos \theta_{t}
$$

and

$$
H_{\|} \cos \theta-H_{\| r} \cos \theta=H_{\| t} \cos \theta_{t},
$$

which can be rewritten as

$$
E_{\perp} \cos \theta-E_{\perp r} \cos \theta=\tilde{n} E_{\perp t} \cos \theta_{t} .
$$

Substituting equation 2.10 into 2.11 , and 2.8 into 2.13 to eliminate the transmitted electric field component $E_{t}$, then taking the ratio of reflected to incident electric field components and substituting the Snell relation $\cos ^{2} \theta_{t}=1-\sin ^{2} \theta / n^{2}$, gives 


$$
\begin{aligned}
& \tilde{r}_{p}=\frac{E_{\| r}}{E_{\|}}=\frac{\tilde{n}^{2} \cos \theta-\sqrt{\tilde{n}^{2}-\sin ^{2} \theta}}{\tilde{n}^{2} \cos \theta+\sqrt{\tilde{n}^{2}-\sin ^{2} \theta}} \\
& \tilde{r}_{s}=\frac{E_{\perp r}}{E_{\perp}}=\frac{\cos \theta-\sqrt{\tilde{n}^{2}-\sin ^{2} \theta}}{\cos \theta+\sqrt{\tilde{n}^{2}-\sin ^{2} \theta}} .
\end{aligned}
$$

The ratio of the Fresnel coefficients, $\rho$, is then defined as

$$
\rho=\frac{\tilde{r_{p}}}{\tilde{r_{s}}}=\tan \psi e^{i \Delta}
$$

where $\Delta$ appears as part of a complex phase shift in relation to $\psi$, which defines the magnitude of the reflectivity. Expressions 2.14 and 2.15 are known as the Fresnel equations and serve as the foundational relationships for all reflectivity probes of the refractive index.

\subsection{Ellipsometric measurement of $\tilde{\epsilon}(\omega)$.}

This section extends our description of light propagation to the optical setup used in the ellipsometry measurement, using refs [36, 37].

\subsubsection{Light polarisation description}

Light is described by plane waves, the electric field vector of which can thus be written in terms of its Cartesian components

$$
\mathbf{E}=\mathbf{E}_{0} \exp [i(k \cdot \mathbf{r}-\omega t)]=\left[\begin{array}{c}
E_{x} \\
E_{y} \\
E_{z}
\end{array}\right] .
$$

The dielectric tensor of a material is defined by

$$
\left(\begin{array}{c}
D_{x} \\
D_{y} \\
D_{z}
\end{array}\right)=\epsilon_{0}\left(\begin{array}{ccc}
\epsilon_{x x} & \epsilon_{x y} & \epsilon_{x z} \\
\epsilon_{y x} & \epsilon_{y y} & \epsilon_{y z} \\
\epsilon_{z x} & \epsilon_{z y} & \epsilon_{z z}
\end{array}\right)\left(\begin{array}{c}
E_{x} \\
E_{y} \\
E_{z}
\end{array}\right),
$$

which, as well as being a diagonalisable matrix (resulting in a tensor for which only the leading diagonals are non zero), has that for an isotropic medium, $\epsilon_{i j} \equiv \epsilon$, so the dielectric tensor collapses to a scalar quantity $\epsilon$, giving

$$
\mathbf{D}=\epsilon_{0} \epsilon \mathbf{E} \text {. }
$$


The $z$-axis is chosen as the direction of propagation of the light, so that the electric field vector in 2.17 can be written as

$$
\left[\begin{array}{c}
E_{x} \\
E_{y} \\
E_{z}
\end{array}\right]=\left(\begin{array}{c}
E_{0 x} \exp [i(k z-\omega t)] \\
E_{0 y} \exp [i(k z-\omega t)] \\
0
\end{array}\right) .
$$

Being concerned, as we are in ellipsometry, with polarisation shifts, we now define a Jones electric field vector for polarisation from the above expression as

$$
\mathbf{E}_{\text {jones }}=\left[\begin{array}{c}
E_{x} \\
E_{y}
\end{array}\right],
$$

omitting the exponential variation of the plane wave which is not polarisation dependent.

For an arbitrary linearly polarised light wave, the electric field vector oscillates along a certain direction in the wavefront inclined to the $x$ axis by an azimuthal angle $\phi$, and may be described by

$$
\mathbf{E}_{\phi}^{\text {jones }}=E_{0}\left[\begin{array}{c}
\cos \phi \\
\sin \phi
\end{array}\right] \text {. }
$$

We now define Jones matrices, $\mathbf{J}^{\text {jones }}$, which describe the effect of optical elements in the experimental setup, pictured in Fig. 2.1, on the polarisation of a light wave. We may then investigate the effect of the optical system as a whole on an input plane wave according to the expression

$$
\mathbf{E}_{\text {out }}^{\text {jones }}=\mathbf{J}^{\text {jones }} \mathbf{E}_{\text {in }}^{\text {jones }},
$$

each of the elements having a particular response function described by $\mathbf{J}^{\text {jones }}$ ${ }^{1}$. The Jones transformation matrices for the different optical elements are now described.

The dielectric material of the ideal polariser is $100 \%$ transmitting for light polarised along its major axis, and $0 \%$ transmitting for light polarised along its minor axis. This is reflected in the matrix below. For simplicity, our description assumes that both the polariser and the analyser are made of

\footnotetext{
${ }^{1}$ Note that Jones formalism requires a well defined polarisation state. This is generally not so for a light source, so the Jones formalism only applies once the beam has passed through the polariser.
} 


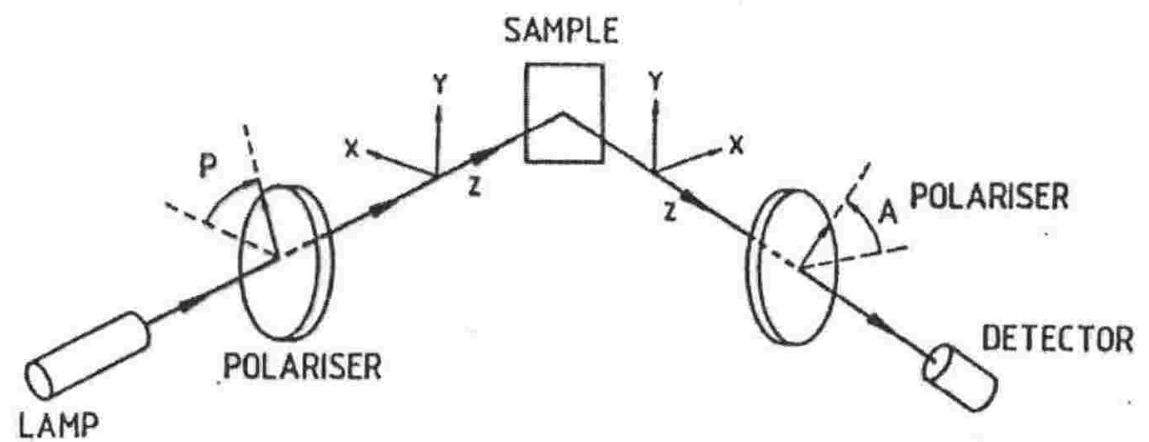

Figure 2.1: Brief schematic of the essential components in the rotating analyser ellipsometry setup. Each of the three optical elements pictured, that is, the polarisers $\mathrm{A}$ and $\mathrm{P}$ and the sample, have a characteristic polarisation response function to the light passing through the system. These response functions, or Jones matrices, may be multiplied to give the total effect of the optical system on the light from the lamp, thereby availing us of a description of the detected light as a function of the optical elements in the system. Diagram courtesy of ref. [36].

ideal polarising material. The inclusion of $\gamma_{p}$, denoting a small ellipticity of the polariser material, introduces cross terms of the electric field. The Jones matrix of the polariser is given by

$$
\mathbf{J}_{p-\text { ellipticity }}=\left(\begin{array}{cc}
1 & -i \gamma_{p} \\
i \gamma_{p} & 0
\end{array}\right)=\left(\begin{array}{ll}
1 & 0 \\
0 & 0
\end{array}\right) \text {. }
$$

An ideal polariser with $\gamma_{p}=0$ has been taken as an approximation for the present results. Upon rotation, the polariser attenuates the electric field components of a non-polarised light beam according to

$$
\mathbf{J}_{\text {polariser }}=\left(\begin{array}{cc}
\cos P & -\sin P \\
\sin P & \cos P
\end{array}\right),
$$

where $P$ denotes the angle the polariser film has rotated through from its major axis of transmission.

The Jones matrix for the sample is

$$
\mathbf{J}_{\text {sample }}=\left(\begin{array}{cc}
r_{p} & 0 \\
0 & r_{s}
\end{array}\right),
$$


adopting the convention that $E_{x}\left(E_{y}\right)$ is the field component in (out of) the plane of incidence. Note that $\mathbf{E}_{\text {out }}=\mathbf{J}_{\text {sample }} \mathbf{E}_{\text {in }}$ gives the expected relations of $r_{p}=E_{\| r} / E_{\|}$and $r_{s}=E_{\perp r} / E_{\perp}$.

In like fashion to the polariser film, we have for the analyser that

$$
\mathbf{J}_{\text {analyser }}=\left(\begin{array}{cc}
\cos A & \sin A \\
-\sin A & \cos A
\end{array}\right),
$$

where $A$ denotes the angle the analyser polarising film has rotated through from its major axis of transmission.

Lastly, the Jones matrix for the analyser film transmission is

$$
\mathbf{J}_{a-\text { ellipticity }}=\left(\begin{array}{cc}
1 & -i \gamma_{a} \\
i \gamma_{a} & 0
\end{array}\right)=\left(\begin{array}{ll}
1 & 0 \\
0 & 0
\end{array}\right),
$$

where $\gamma_{a}$ denotes a small ellipticity of the polariser material in the analyser. Again, an ideal polariser has been taken as an approximation for the present results so that $\gamma_{a}=0$.

The effect of any optical configuration on a plane wave passing through it may be described by a product of Jones matrices, and the Jones matrices introduced here are the only descriptive components required for the rotating analyser ellipsometer discussed in the next section.

\subsubsection{Rotating analyser ellipsometer}

The electric field output to the detector of the rotating analyser ellipsometer is described by the following expression using the elements described in the previous section;

$$
\begin{aligned}
& \mathbf{E}_{\text {det }}=\mathbf{J}_{\text {p-ellipticity }} \mathbf{J}_{\text {polariser }} \mathbf{J}_{\text {sample }} \mathbf{J}_{\text {analyser }} \mathbf{J}_{\text {a-ellipticity }} \mathbf{E}_{\text {in }} \\
& =\left(\begin{array}{ll}
1 & 0 \\
0 & 0
\end{array}\right)\left(\begin{array}{cc}
\cos A & \sin A \\
-\sin A & \cos A
\end{array}\right)\left(\begin{array}{cc}
r_{p} & 0 \\
0 & r_{s}
\end{array}\right) \\
& \times\left(\begin{array}{cc}
\cos P & -\sin P \\
\sin P & \cos P
\end{array}\right)\left(\begin{array}{ll}
1 & 0 \\
0 & 0
\end{array}\right) \mathbf{E}_{0} \\
& =\left(\begin{array}{ll}
r_{p} \cos P \cos A+r_{s} \sin P \sin A & 0
\end{array}\right)\left(\begin{array}{c}
E_{x} \\
E_{y}
\end{array}\right),
\end{aligned}
$$

substituting in the vector relationship for $E_{0}$. From this expression we find the intensity of light at the detector by recalling that $I_{\text {det }} \propto\left|\mathrm{E}_{\text {det }}\right|^{2}$, and thus 
get

$$
I_{\text {det }} \propto 1+\alpha(\tilde{\rho}, P) \cos (2 A)+\beta(\tilde{\rho}, P) \sin (2 A),
$$

where $A$ is made to vary during the experiment and $\alpha(\tilde{\rho}, P)$ and $\beta(\tilde{\rho}, P)$ are the Fourier coefficients

$$
\alpha(\tilde{\rho}, P)=\frac{|\tilde{\rho}|^{2} \cos ^{2} P-\sin ^{2} P}{|\tilde{\rho}|^{2} \cos ^{2} P+\sin ^{2} P}
$$

and

$$
\beta(\tilde{\rho}, P)=\frac{\operatorname{Re}(\tilde{\rho}) \sin (2 P)}{|\tilde{\rho}|^{2} \cos ^{2} P+\sin ^{2} P} .
$$

Recalling that $\tilde{\rho}=\tan \psi e^{i \Delta}=r_{p} / r_{s}$, we can extract $\psi$ and $\Delta$ from the above expressions.

A measurement of the intensity variation with $A$ yields a sinusoidal curve (refer to the bottom panel of Fig. 3.6) which can be fitted to equation 2.31. This enables the fourier coefficients $\alpha, \beta$, and thus $\psi$ and $\Delta$ to be extracted via a Fourier transform. Once these have been found, the complex reflectance ratio can be calculated using equation 2.16 , and the dielectric constant found using

$$
\epsilon=\sin ^{2} \theta+\sin ^{2} \theta \tan ^{2} \theta\left[\frac{1-\rho}{1+\rho}\right]^{2},
$$

where $\theta$ is the angle of incidence of the light beam. There are two things to note in particular about this formalism. Firstly, the dielectric constant is directly calculable from the equations outlined above. Secondly, the fourier coefficients $\alpha$ and $\beta$ are directly obtained from equation 2.31, and their determination is independent of the intensity of the beam. As well as this technique being, in principle, insensitive to fluctuations in the input beam, it importantly does not require the determination of the absolute reflectivity of a sample.

\subsubsection{Reduction of ellipsometric constants.}

The expressions outlined above are derived upon the assumption that the light is incident on an isotropic medium, that is, one in which the dielectric constant is independent of the crystal direction and $\epsilon=\epsilon_{x x}=\epsilon_{y y}=\epsilon_{z z}$, 
as seen in equation 2.19. For the $n=2$ Ruddlesden-Popper series of crystals, to which the ellipsometry measurements in this project are devoted, the lattice is uniaxially anisotropic (the $a$ and $b$ axes are regarded as interchangeable, but the $c$-axis differs from them both), and the assumption of isotropy becomes problematic. In the instance of calculating the dielectric constant of an anisotropic material from its reflection intensity, if using the above formalism for isotropic materials, the result is the so-called pseudodielectic function[37]. When referring to the measurement from the $a b(c a)$ plane we will term the pseudo-dielectric function as the $a b-c(c-a b)$ response. This conveys the fact that the $c$-axis ( $a b$ plane) response is not yet decoupled from that of the face measured. If the mixing between $a b$ and $c$ responses is significant, the $a b-c(c-a b)$ pseudo-dielectric function will be a poor approximation to the dielectric function for the $a b$ plane $(c \text {-axis })^{2}$. The approach to disentangling the $a b$ and $c$ dielectric responses is to reduce the ellipsometric constants assuming isotropy, and then to fit for an anisotropic correction. This anistropic fitting correction is done according to the following [37, 39].

We again assume that the light is incident at an angle $\theta$ from an isotropic medium, with dielectric constant $\tilde{n}_{0}=1$, onto a uniaxially anisotropic crystal. Assuming there is no anisotropy in the $a b$ plane, we choose $\tilde{n}_{\|}\left(\sqrt{\epsilon_{x x, y y}}\right)$ and $\tilde{n}_{\perp}\left(\sqrt{\epsilon_{z z}}\right)$ to denote the principal components of the refractive index, $\tilde{n}_{\|}$referring to the in-plane $(a b)$ component, and $\tilde{n}_{\perp}$ referring to the out of plane $(c)$ component. As illustrated in Fig. 2.2, we consider two different orientations of the $c$-axis with respect to the sample surface and plane of incidence, corresponding to the two different sample orientations used in the experiments here. Firstly, with the $c$-axis perpendicular to the surface and using the boundary conditions and a similar analysis to that used in section 2.1.2, we obtain the following relations for the reflection coefficients of $s$ and

\footnotetext{
${ }^{2} a b$ coupling to $c$-axis longitudinal optic phonons has even reportedly been observed, and is seen as a "notch"-like feature in a normal incidence reflectance measurement of high $T_{c}$ superconductors[38].
} 


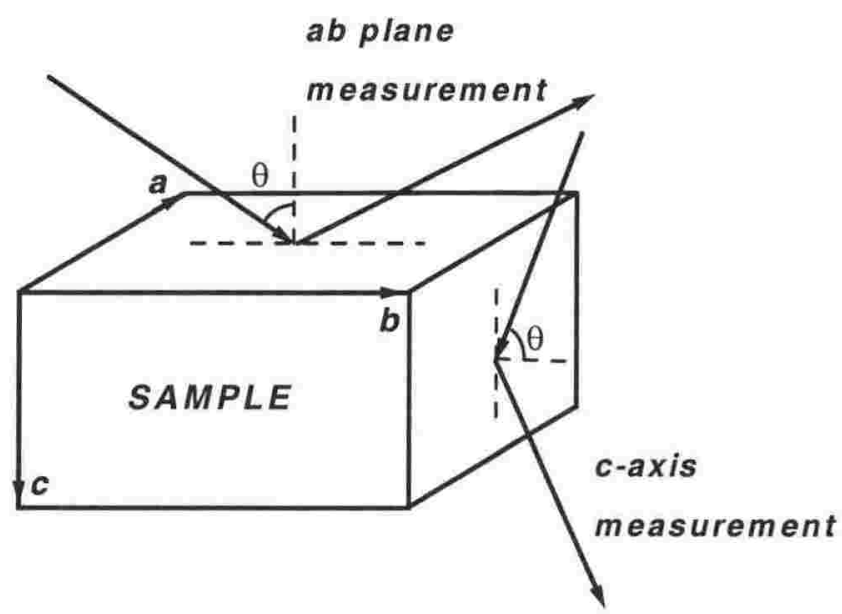

Figure 2.2: Schematic diagram showing the geometry of the $a b$ plane and $c$-axis ellipsometry measurements with respect to the uniaxially anisotropic sample.

$p$ polarised light: ${ }^{3}$

$$
\begin{gathered}
r_{p}^{\perp}=\frac{\tilde{n}_{\perp} \tilde{n}_{\|} \cos \theta-\sqrt{\tilde{n}_{\|}^{2}-\sin ^{2} \theta}}{\tilde{n}_{\perp} \tilde{n}_{\|} \cos \theta+\sqrt{\tilde{n}_{\|}^{2}-\sin ^{2} \theta}} \\
r_{s}^{\perp}=\frac{\cos \theta-\sqrt{\tilde{n}_{\|}^{2}-\sin ^{2} \theta}}{\cos \theta+\sqrt{\tilde{n}_{\|}^{2}-\sin ^{2} \theta}} .
\end{gathered}
$$

We have chosen here to use the superscript $\perp$ on $r_{p}$ and $r_{s}$ to refer to the particular orientation of the $c$-axis considered here, that is, perpendicular to the sample-air interfacial plane.

Now, if the $c$-axis is oriented parallel to the sample surface, and also lies in the plane of incident light, the analysis from section 2.1 .2 yields

$$
r_{p}^{\|}=\frac{\tilde{n}_{\perp} \tilde{n}_{\|} \cos \theta-\sqrt{\tilde{n}_{\perp}^{2}-\sin ^{2} \theta}}{\tilde{n}_{\perp} \tilde{n}_{\|} \cos \theta+\sqrt{\tilde{n}_{\perp}^{2}-\sin ^{2} \theta}}
$$

\footnotetext{
${ }^{3}$ Note that the cross polarised components of the reflected light $r_{p s}=r_{s p}=0$.
} 


$$
r_{s}^{\|}=\frac{\cos \theta-\sqrt{\tilde{n}_{\perp}^{2}-\sin ^{2} \theta}}{\cos \theta+\sqrt{\tilde{n}_{\perp}^{2}-\sin ^{2} \theta}}
$$

where the superscript $\|$ has been chosen to denote the present situation of the $c$-axis at the intersection of the sample surface and the incident plane. We omit the case of the $c$-axis parallel to the sample surface yet perpendicular to the plane of incidence, as samples were not measured in this geometry here. The interested reader is referred to Aspnes and coworkers[40] for more detail.

Using equations 2.35 and 2.36 we find that the reflection coefficient for the orientation whereby the $c$-axis is perpendicular to the reflecting surface is

$$
\rho^{\perp}=\frac{\left(\tilde{n}_{\perp} \tilde{n}_{\|} \cos \theta-\sqrt{\tilde{n}_{\|}^{2}-\sin ^{2} \theta}\right)\left(\cos \theta+\sqrt{\tilde{n}_{\|}^{2}-\sin ^{2} \theta}\right)}{\left(\tilde{n}_{\perp} \tilde{n}_{\|} \cos \theta+\sqrt{\tilde{n}_{\|}^{2}-\sin ^{2} \theta}\right)\left(\cos \theta-\sqrt{\tilde{n}_{\|}^{2}-\sin ^{2} \theta}\right)} .
$$

Likewise, using equations 2.37 and 2.38 gives

$$
\rho^{\|}=\frac{\left(\tilde{n}_{\perp} \tilde{n}_{\|} \cos \theta-\sqrt{\tilde{n}_{\perp}^{2}-\sin ^{2} \theta}\right)\left(\cos \theta+\sqrt{\tilde{n}_{\perp}^{2}-\sin ^{2} \theta}\right)}{\left(\tilde{n}_{\perp} \tilde{n}_{\|} \cos \theta+\sqrt{\tilde{n}_{\perp}^{2}-\sin ^{2} \theta}\right)\left(\cos \theta-\sqrt{\tilde{n}_{\perp}^{2}-\sin ^{2} \theta}\right)}
$$

for the $c$-axis at the intersection of the incident and sample surface planes.

In order to extract $\tilde{n}_{\|}$and $\tilde{n}_{\perp}$, the principal components of the dielectric tensor, a MATLAB computer programme written by Andrzej Golnik, fits measured data $\rho^{\|}$and $\rho^{\perp}$ to equations 2.39 and 2.40. Comparisons between $a b-c(c-a b)$ and $a b(c)$ dielectric functions, and uncertainties due to the reduction of the pseudo-dielectric constants, will be discussed in Appendix A.

\subsubsection{Deduction of $c$-axis conductivity with $a b$ plane measurements.}

We noted, in the previous section, that the dielectric function of a particular axis of an anisotropic material, can be quite different from the pseudodielectric function measured for the sample in that orientation. The differences are so marked for the present samples studied, in fact, that they can be seen immediately, by observation of the $a b-c$ pseudo-dielectric functions 
for $\mathrm{PrSr}_{2} \mathrm{Mn}_{2} \mathrm{O}_{7}$ and $\mathrm{La}_{1.2} \mathrm{Sr}_{1.8} \mathrm{Mn}_{2} \mathrm{O}_{7}$ displayed in appendix A. Fig. 2.3 displays light rays in the normal incidence probe (to be discussed in the next section) and the ellipsometry measurement on the $a b$ plane. It can be seen that the transverse component of the electric field, $E_{T}$, probes the sample at a different angle for each experiment. Due to $E_{T}$ being near parallel to the $c$ axis, the reflected beam in the oblique incidence probe contains a significant $c$-axis response. In the particular case of our ellipsometry measurements, in the geometry whereby the $c$-axis is perpendicular to the surface, an eyeball comparison between the $a b-c$ pseudo-dielectric function, or $\rho^{\perp}$, and the $a b$ plane normal incidence reflectance measurements, show differences in certain spectral regions that must be ascribed to $c$-axis excitation of the probing field.

It was suggested, by H. J. Trodahl, that we may exploit the discrepancy and deduce the $c$-axis dielectric constant, or $n_{\perp}$, from $a b-c$ plane ellipsometry (or $\rho^{\perp}$ ) and $a b$ plane normal incidence reflectivity measurements. This, of course, relies on there being some out of plane component interacting with the electric field vector in the oblique incidence ellipsometry measurement. We perform the calculations and demonstrate here that, in theory, not only is the deduction of $c$-axis optical constants from $a b$ plane measurements possible, but that the process is even relatively straightforward.

We make the assumption that the normal incidence infrared reflectivity method, with appropriate extrapolations, gives the $a b$ plane dielectric constant, or $n_{\|}$, exclusively.

We can rearrange equation 2.39 so that the refractive index, $n_{\perp}$, of the $c$-axis of the material is expressed in terms of $n_{\|}$by

$$
\frac{\tilde{n}_{\perp}}{\sqrt{\tilde{n}_{\perp}^{2}-\sin ^{2} \theta}}=\frac{C+\rho^{\perp}}{\tilde{n}_{\|} \cos \theta\left(C-\rho^{\perp}\right)}=N_{1},
$$

where the quantity $N_{1}$ is a label chosen for the middle part of equation 2.41 and

$$
C=\frac{\cos \theta+\sqrt{\tilde{n}_{\|}^{2}-\sin ^{2} \theta}}{\cos \theta-\sqrt{\tilde{n}_{\|}^{2}-\sin ^{2} \theta}} .
$$

The complex frequency-dependent constant $N_{1}$ may be directly calculated 


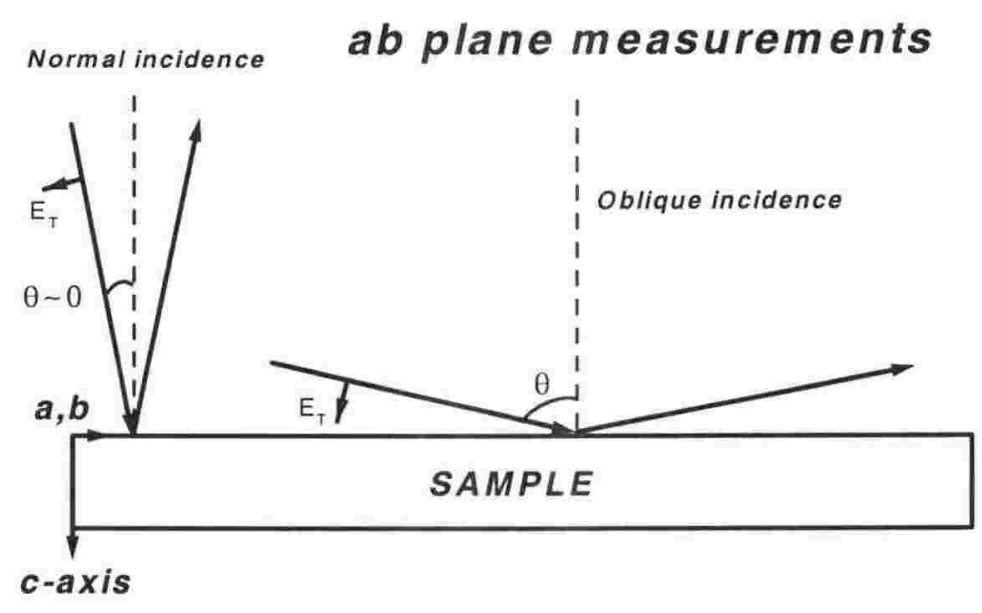

Figure 2.3: Schematic of the normal and oblique incidence measurements on the sample surface.

given that, in this experiment, $\theta, \tilde{n}_{\|}$and $\rho^{\perp}$ are known. $\theta$ is the angle of incidence used in the ellipsometry experiment, $\tilde{n}_{\|}$is the dielectric constant for the $a b$ plane, deduced by Kramers-Kronig transform of the measured normal incidence reflectance (which will be introduced in section 2.3), and $\rho^{\perp}$ is the reflection coefficient measured from the $a b$ plane by oblique incidence ellipsometry.

$\tilde{n}_{\perp}$ is then found most exeditiously by minimising $a$ in the function

$$
a=\frac{\tilde{n}_{\perp}}{\sqrt{\tilde{n}_{\perp}^{2}-\sin ^{2} \theta}}-N_{1}
$$

at each frequency. This is attempted using a MATLAB routine for the two steps of calculation and minimisation.

In practice there are large errors associated with this method if the $c$-axis dielectric response does not greatly interfere with the ellipsometric reflection coefficient $\rho^{\perp}$. We present details of the attempted reduction in section 4.4. 


\subsection{Normal Incidence reflectance measurement of $\tilde{\epsilon}(\omega)$}

In the previous section, expressions were given for the reflectivity of the $p$ and $s$ polarisations of light incident on a material at a given angle $\theta$. For normally incident light, $\theta=0$, Fresnel's equations collapse to

$$
r_{p}=r_{s}=\frac{1-\tilde{n}}{1+\tilde{n}}=\tilde{r} .
$$

The reflectance, $R=\tilde{r} \tilde{r}^{*}$ of a surface is thus (recalling that $\tilde{n}=n+i k$ )

$$
R=\frac{(n-1)^{2}+k^{2}}{(n+1)^{2}+k^{2}}
$$

Using $\epsilon_{1}=n^{2}-k^{2}$ and $\epsilon_{2}=2 n k$ the reflectance can be found in terms of $\epsilon_{1}$ and $\epsilon_{2}$, which are related to $n$ and $k$ by the following relations:

$$
n=\left\{\frac{1}{2}\left[\sqrt{\epsilon_{1}^{2}+\epsilon_{2}^{2}}+\epsilon_{1}\right]\right\}^{1 / 2}
$$

and

$$
k=\left\{\frac{1}{2}\left[\sqrt{\epsilon_{1}^{2}+\epsilon_{2}^{2}}-\epsilon_{1}\right]\right\}^{1 / 2} .
$$

\subsubsection{Deduction of $\tilde{\epsilon}(\omega)$ from $R(\omega)$.}

We would like to find $n$ and $k$ using our measured normal incidence reflectance $R$, however it is impossible to uniquely determine $\tilde{n}$ from equation 2.45 . It is possible however to extract the real and imaginary components of the dielectric constant of a material by using dispersion relations. These relate a dispersive process to an absorptive process, for instance $n \rightarrow k$, and are also used to derive sum rules. Dispersion relations follow from the principle of causality, a system cannot respond to an optical stimulus before that stimulus has interacted with the material.

Fig. 2.4 illustrates the relationship between causality and dispersion relations. Consider an input (A) which is made up many different fourier components, of which (B) is one, all combining to destructively interfere before the arrival of that light, that is for $t<0$. Now if a material interacting with 

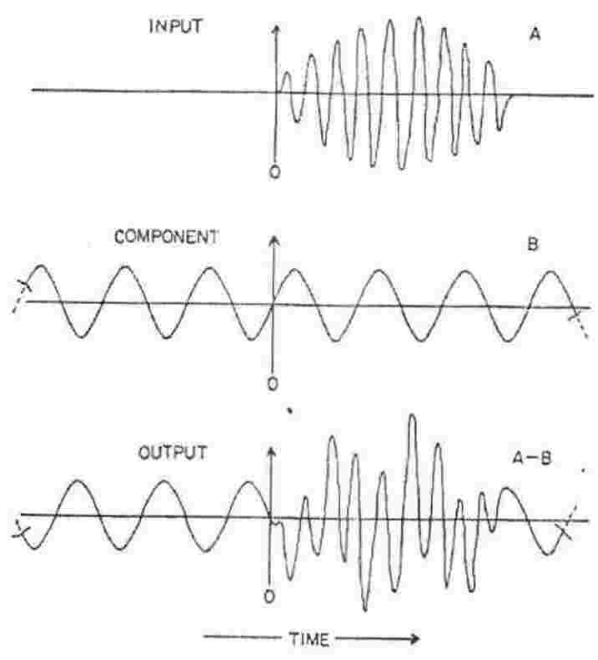

Figure 2.4: Schematic representation of the connection between causality and the dispersion relations. Diagram reproduced from Toll[42].

the input for $t>0$, were to absorb just the frequency of the (B) component of the input wave packet, then we would have (A-B), the signal represented by the bottom figure of fig. 2.4. However, this signal has a $-\mathrm{B}$ component in the $t<0$ region, implying the input wave interacted with the material before it even arrived, in contradiction with causality. In order to account for this then, there must be an accompanying phase shift of the other frequencies of the input upon absorption of a particular component.

We now set about an illustration of the origin of the dispersion relations for the complex reflectivity, which is given by

$$
\tilde{r}(\omega)=\rho(\omega) e^{i \theta(\omega)}
$$

where $\rho(\omega)$ is the reflectivity amplitude and $\theta(\omega)$ is the phase shift.

Equation 2.48 is now written in the more convenient form, separating out the real and imaginary parts,

$$
\ln \tilde{r}=\ln \rho(\omega)+i \theta(\omega) .
$$

The time-dependent variable $\tilde{r}(t)$ is taken to introduce the causality condition to our analysis. It and its frequency-dependent counterpart are related 
by the Fourier transform,

$$
\ln \tilde{r}(\omega)=\int_{-\infty}^{\infty} \ln \tilde{r}(t) e^{i \omega t} d t .
$$

Then allowing the frequency to be complex, that is, $\tilde{\omega}=\omega_{1}+i \omega_{2}$ gives

$$
\ln \tilde{r}(\tilde{\omega})=\int_{-\infty}^{\infty} \ln \tilde{r}(t) e^{i \omega_{1} t} e^{-\omega_{2} t} d t .
$$

The $e^{i \omega_{1} t}$ term is bounded for all frequencies, but the integral diverges by virtue of the second exponential, for $\omega_{2}<0$. Now $\ln \tilde{r}(\tilde{\omega})$ is only defined for $t>0$, due to causality. The integral, thus, must be worked in the upper half frequency plane only, where $\omega_{2} \geq 0$.

Two important conditions that must be met for this and the following analysis are dicussed in Appendix G of Wootten[43]. Firstly, it is established there that the contribution to the integral becomes vanishingly small as the frequency $\rightarrow \infty$. This ensures that the integral is non-diverging and demonstrates that our measured quantities, $\ln \rho(\omega)$ and $\theta(\omega)$, are causal and therefore linked by dispersion relations. Secondly, $\ln \tilde{r}(\tilde{\omega})$ is shown to not have any poles. It is important to establish that there are no singularities in the response function, as mathematically these imply that an effect is seen in the absence of a stimulus.

Having established that the assumptions above are met, Cauchy's theorem can be applied to the complex reflectivity, $\tilde{r}$, as follows:

$$
\ln \tilde{r}(\omega)=\frac{1}{\pi i} \wp \int_{-\infty}^{\infty} \frac{\ln \tilde{r}\left(\omega^{\prime}\right)}{\omega^{\prime}-\omega} d \omega^{\prime},
$$

where $\wp$ denotes the principal value, and $\omega^{\prime}$ and $\omega$ are the integration and the ranging frequencies respectively. The real and imaginary parts of 2.52 lead respectively to the dispersion expressions

$$
\ln \rho(\omega)=\frac{1}{\pi} \wp \int_{-\infty}^{\infty} \frac{\theta\left(\omega^{\prime}\right)}{\omega^{\prime}-\omega} d \omega^{\prime}
$$

and

$$
\theta(\omega)=-\frac{1}{\pi} \wp \int_{-\infty}^{\infty} \frac{\ln \rho\left(\omega^{\prime}\right)}{\omega^{\prime}-\omega} d \omega^{\prime}
$$

relating $\rho(\omega)$ and $\theta(\omega)$. 
Now, imposing the conditions that $\tilde{r}(t)=\tilde{r} *(t)$ and $\tilde{r}(-\omega)=\tilde{r} *(\omega)$, we may restrict the integral to positive values of $\omega^{\prime}$, thereby giving

$$
\theta(\omega)=-\frac{2 \omega}{\pi} \wp \int_{0}^{\infty} \frac{\ln \rho\left(\omega^{\prime}\right)}{\left(\omega^{\prime}\right)^{2}-\omega^{2}} d \omega^{\prime} .
$$

This is known as the Kramers Kronig dispersion relation for $\theta(\omega)$. Having measured $\rho(\omega)$ at a large range of frequencies, and made appropriate extrapolations to 0 and $\infty$, it is possible to determine the phase, and thus $\tilde{r}(\omega)$, from equation 2.48 , by using the above expression.

\subsubsection{Sum rules}

The Kramers-Kronig dispersion relations for $\tilde{\epsilon}$, by a similar analysis to that in the previous subsection, are

$$
\epsilon_{1}(\omega)-1=\frac{2}{\pi} \wp \int_{0}^{\infty} \frac{\omega^{\prime} \epsilon_{2}\left(\omega^{\prime}\right)}{\left(\omega^{\prime}\right)^{2}-\omega^{2}} d \omega^{\prime}
$$

and

$$
\epsilon_{2}(\omega)=-\frac{2 \omega}{\pi} \wp \int_{0}^{\infty} \frac{\epsilon_{1}\left(\omega^{\prime}\right)-1}{\left(\omega^{\prime}\right)^{2}-\omega^{2}} d \omega^{\prime} .
$$

This enables the extraction of sum rules using these dispersion relations. Without justification (see Wootten[43] for further detail) the sum rule expression for finding the effective number of charge carriers from the integrated conductivity of a material is

$$
N_{e f f}(\omega)=\frac{2 m V}{\pi N e^{2}} \int_{0}^{\omega_{c}} \sigma_{1}\left(\omega^{\prime}\right) d \omega^{\prime},
$$

where $V$ is the volume of a unit cell, $m$ and $e$ are the carrier mass and charge respectively and $N$ is the number of (in this case) Mn atoms in that unit cell which contribute as carrier host sites.

\subsubsection{Reflectance extrapolations.}

The reflectance is measured over a finite wavelength range but, in order to extract the real and imaginary parts of the dielectric constant, one requires that the reflectance be known for all energies, as described in the previous section. 
Because it is not possible to measure the reflectivity at all frequencies, we must use appropriate extrapolations in the regions beyond our data. This section will discuss what constitutes an appropriate extrapolation. Although not immediately apparent from the Kramers-Kronig relations in equations 2.56 and 2.57, abrupt changes in the reflectivity data can affect the transform output in spectral regions far removed from the changes in $R(\omega)$. It is crucial that extrapolations at the data extremes are chosen wisely, as they have the potential to significantly affect the transform well within the regions of data itself. The effect of varying extrapolations should be closely monitored for a guide to the uncertainty brought about by them. When experimental data is put forth in Chapter 4, the uncertainty associated with extrapolations will be discussed further in this context.

For materials of a metallic nature, the low frequency extrapolation incorporating the "Hagen-Rubens" approximation may be applicable. Substances with a high conductivity have $\epsilon_{2} \gg \epsilon_{1}$, that is, the absorptive part of the dielectric constant is much greater than the inductive part. This means that $\tilde{n}^{2} \approx i \epsilon_{2}$, and from this we find that $n^{2}-k^{2} \approx 0$ and $2 n k=\epsilon_{2}=4 \pi \sigma_{1} / \omega$. Thus, in the limit of $\epsilon_{2} \gg \epsilon_{1}$,

$$
n \approx k \approx \sqrt{\frac{2 \pi \sigma_{1}}{\omega}} .
$$

The Hagen-Rubens relation for the reflectivity, at energies where $\epsilon_{2} \gg \epsilon_{1}$, follows by substituting equation 2.59 into equation 2.45 , giving

$$
R(\omega)=1-2 \sqrt{\frac{\omega}{2 \pi \sigma_{1}}} .
$$

Thus, for free electron materials, the zero energy reflectance is equal to 1. In the energy region for which $\sigma_{1} \gg \sigma_{2}, R(\omega)$ varies as $\omega^{1 / 2}$, and is determined solely by the real part of the conductivity, $\sigma_{1}$.

For non-metallic materials the appropriate low energy extrapolation is to assume that the reflectivity magnitude at the lowest measured frequency extrapolates to zero frequency. This is made to be consistent with the Lorentz oscillator reflectivity, seen in the next section, for which the tail of the absorption peak has some finite reflectance ratio at zero frequency. 
For our high energy extrapolations we assume free electron behaviour, and thus applicability of the response varying as $R(\omega) \propto \omega^{-4}$ [43]. The energy at which this approximation begins to apply is generally taken as $36 \mathrm{eV}$ and above. This energy is far enough removed from the high-energy end of the data that the extrapolation has little impact on the Kramers-Kronig transform in the region of data.

\subsection{Optical response of a harmonic oscillator.}

The mathematical formalism of describing the most simple absorptive components; a bound oscillator and a free oscillator, will be introduced in this section in the context of modelling the optical absorption of a material's oscillators.

\subsubsection{The bound charge response}

A very simple and yet powerful model for the behaviour of an electron in the presence of a nucleus is put forward in this section. The response is that of a damped harmonic oscillator,

$$
m \frac{d^{2} \tilde{r}}{d t^{2}}+m \Gamma \frac{d \tilde{r}}{d t}+m \omega_{0}^{2} \tilde{r}=-e \mathbf{E}
$$

where $m$ and $e$ are, respectively, the mass and charge of a bound electron/carrier, (the mass of the nucleus has been taken as infinite) $\omega_{0}$ is the resonant frequency, and $\mathrm{E}$ is the local field experienced by that bound carrier. The second and third terms in equation 2.61 are an energy damping term and the Hooke's law restoring force respectively.

The field can be taken to vary as $e^{-i \omega t}$ giving a solution to the equation of motion in 2.61 as

$$
\tilde{x}=\frac{-e \mathbf{E} / m}{\left(\omega_{0}^{2}-\omega^{2}\right)-i \Gamma \omega} .
$$

The induced dipole moment for the charge $e$ is $\tilde{p}=-e \tilde{x}$, which gives

$$
\tilde{p}=\frac{e^{2} \mathbf{E}}{m} \frac{1}{\left(\omega_{0}^{2}-\omega^{2}\right)-i \Gamma \omega} .
$$




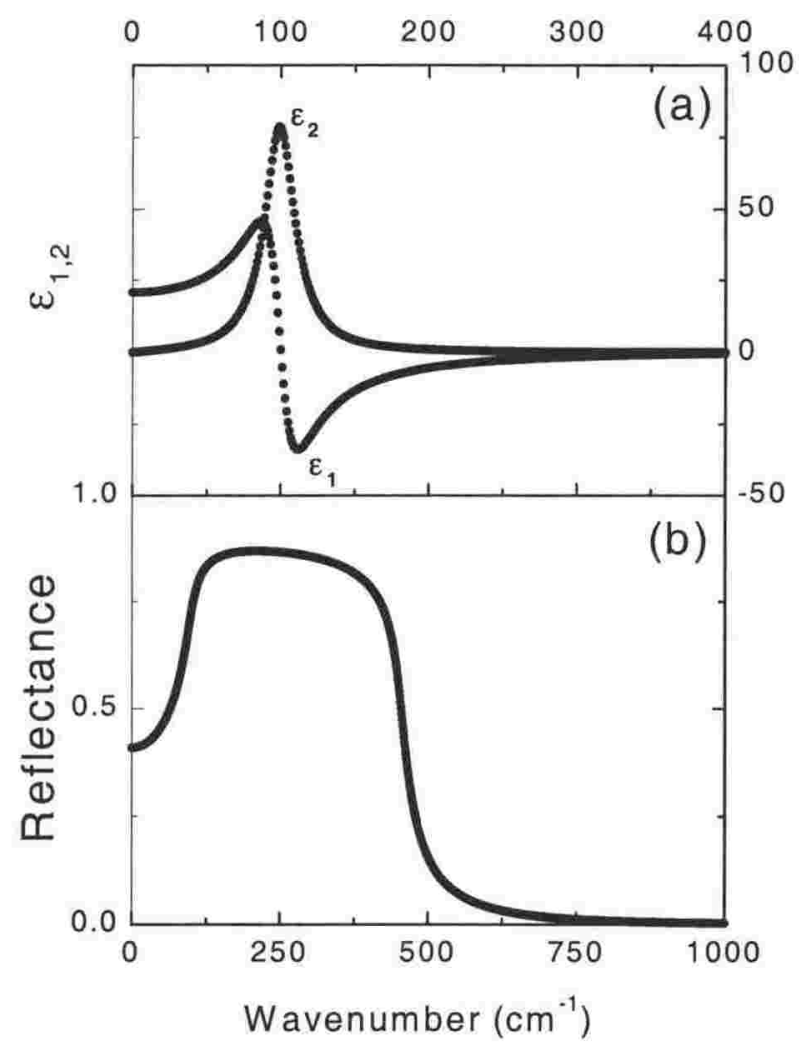

Figure 2.5: Example $\epsilon_{1}(\omega), \epsilon_{2}(\omega)$ (a) and reflectance, $R(\omega)$ (b) for the Lorentz harmonic oscillator. Note the change in $x$-axis scale between the top and bottom panels.

Assuming a linear relationship between $\tilde{p}$ and $\mathbf{E}$, that is, $\tilde{x}$, and that the displacement of the charge from its equilibrium position is small and the process is elastic, enables us to write $\tilde{p}=\tilde{\alpha}(\omega) \mathbf{E}$, where $\tilde{\alpha}(\omega)$ is the frequency dependent atomic polarisability.

Now, recalling that

$$
\tilde{\epsilon}=1+4 \pi N \tilde{\alpha}
$$

for small $N \tilde{\alpha}$, we get

$$
\tilde{\epsilon}=1+\frac{4 \pi N e^{2}}{m} \frac{1}{\left(\omega_{0}^{2}-\omega^{2}\right)-i \Gamma \omega} .
$$

Thus, the real and imaginary components of the dielectric constant for 
nonmagnetic materials, commonly referred to as the Lorentz oscillator dielectric, are

$$
\begin{gathered}
\epsilon_{1}=1+\frac{4 \pi N e^{2}}{m} \frac{\left(\omega_{0}^{2}-\omega^{2}\right)}{\left(\omega_{0}^{2}-\omega^{2}\right)^{2}+\Gamma^{2} \omega^{2}} \\
\epsilon_{2}=\frac{4 \pi N e^{2}}{m} \frac{\Gamma \omega}{\left(\omega_{0}^{2}-\omega^{2}\right)^{2}+\Gamma^{2} \omega^{2}} .
\end{gathered}
$$

Panels (a) and (b) of Fig. 2.5 respectively display example $\epsilon_{1}$ and $\epsilon_{2}$, as well as the reflectivity calculated from the example dielectric constant, using equations 2.45, 2.46 and 2.47. This response is typical of that of an insulator, and Lorentzian signatures are visible throughout optical conductivity spectra in the form of phonon oscillations and electron transitions. Countless spectra for insulating materials have been successfully modelled using the described formalism.

\subsubsection{The Drude response.}

The Drude response follows very naturally from that of the Lorentz oscillator. Because it describes the behaviour of a free electron, the only modification to the harmonic oscillator theory already described is to set the restoring force, or the third term in equation 2.61 , to zero. This readily gives the expressions:

$$
\epsilon_{1}=1-\frac{4 \pi N e^{2}}{m} \frac{1}{\omega^{2}+\Gamma^{2}}
$$

and

$$
\epsilon_{2}=\frac{4 \pi N e^{2}}{m} \frac{\Gamma}{\omega\left(\omega^{2}+\Gamma^{2}\right)}
$$

for the real and imaginary parts of the Drude dielectric function.

Again, panels (a) and (b) of Fig. 2.6 respectively display example $\epsilon_{1}$ and $\epsilon_{2}$, as well as the reflectivity expected for a Drude material. The metallic response functions, $\epsilon_{1}$ and $\epsilon_{2}$, are respectively characterised by diverging negative and positive values as $\omega \rightarrow 0$. The reflectivity is high in the far-IR, approaching a value of $R=1$ at $\omega=0$.

To this point the discussion has been of the dielectric constant and refractive index, but the spectra which will be presented are predominantly those of the optical conductivity, $\tilde{\sigma}(\omega)=\sigma_{1}(\omega)+i \sigma_{2}(\omega)$. In section 2.1.1 we found 


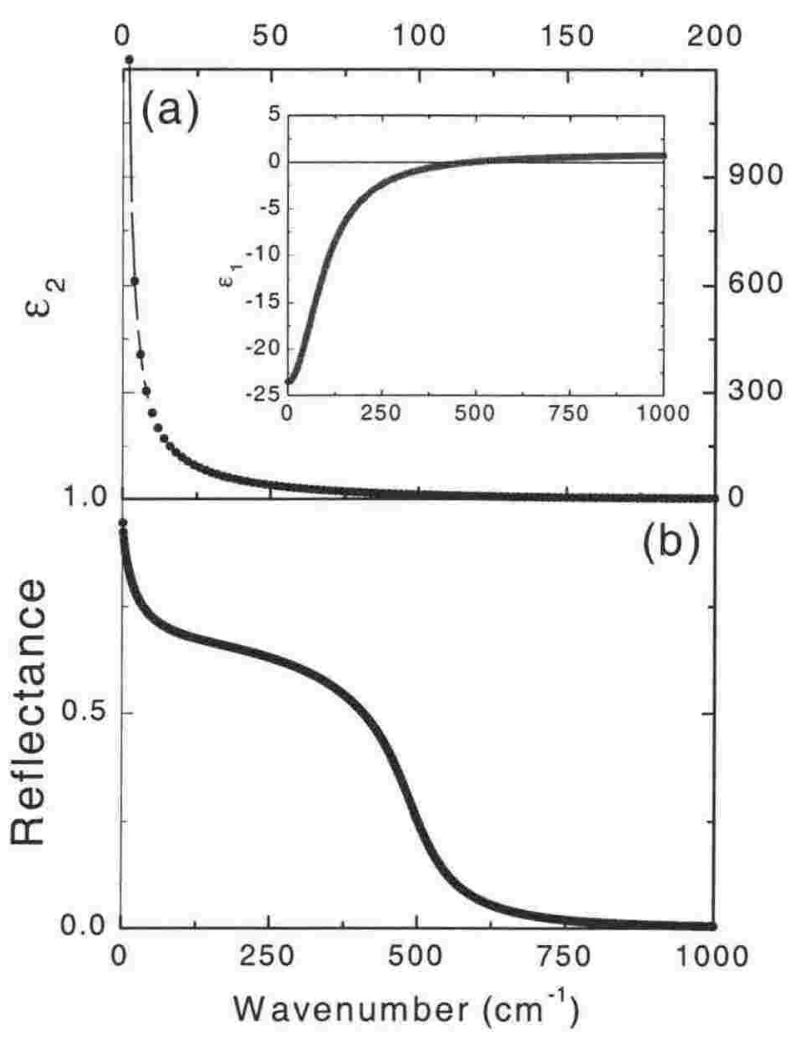

Figure 2.6: Example $\epsilon_{1}(\omega)$ (inset), $\epsilon_{2}(\omega)$ (a) and reflectance, $R(\omega)$ (b) for the Drude oscillator response. Note the changes in $x$-axis scale between the inset, top and bottom panels.

that $4 \pi \sigma_{1} / \omega=2 n k / \mu$ or $\sigma_{1}=\omega \epsilon_{2} / 4 \pi$. We can extend this relationship into the more general identity

$$
\epsilon_{1}+i \epsilon_{2}=\frac{\omega+4 \pi i\left(\sigma_{1}+i \sigma_{2}\right)}{\omega}
$$

The most often presented spectra will be of the real or absorptive part of the conductivity, $\sigma_{1}(\omega)$, and the inductive part of the dielectric constant, $\epsilon_{1}(\omega)$. 


\subsubsection{Phonon oscillators}

The $A_{1-x} B_{x} \mathrm{MnO}_{3}$ perovskite manganite can have cubic, orthorhombic or rhombohedral symmetry depending on the substitution level and type of cation on the $B$ site. The crystal symmetries, along with the Wyckoff sites occupied by the different ions in the manganites, enable the determination of infrared and raman active modes by group theory. The irreducible representations from factor group analysis were found for each of the structural types of manganite presented here using the process detailed in Rousseau and coworkers[44] and x-ray crystallography tables[45]. For the material with cubic symmetry, or the $O_{h}^{1}(\mathrm{Im} 3 \mathrm{~m})$ space group and site occupancy as given in ref.[46], the irreducible representation is

$$
4 F_{1 u}+F_{2 u},
$$

of which the acoustic mode is $F_{1 u}, F_{2 u}$ is silent leaving $3 F_{1 u}$ modes that are infrared (IR) active[47].

The irreducible representation of the material with orthorhombic symmetry $D_{2 h}^{16}(\mathrm{mmm})$, and site occupancy as found in ref.[48], is

$$
7 A_{g}+5 B_{1 g}+7 B_{2 g}+5 B_{3 g}+8 A_{u}+10 B_{1 u}+8 B_{2 u}+10 B_{3 u},
$$

of which the $A_{g}, B_{1 g}, B_{2 g}$ and $B_{3 g}$ modes are Raman active, the $A_{u}$ modes are silent, the acoustic modes are $B_{1 u}, B_{2 u}$ and $B_{3 u}$, leaving the IR active modes $9 B_{1 u}, 7 B_{2 u}$ and $9 B_{3 u}[48]$.

None of the samples presented used in this work are of rhombohedral symmetry, but the reader is referred to [47] if interested in the irreducible representations of the manganites with such a structure.

The irreducible representation of the $(R E / \mathrm{Sr})_{3} \mathrm{Mn}_{2} \mathrm{O}_{7} n=2$ layered structure, with $I / 4 \mathrm{mmm}$ or $D_{4 h}^{17}$ tetragonal symmetry and Wyckoff sites as given in ref.[49], is

$$
6 A_{2 u}+7 E_{u}+4 A_{1 g}+5 E_{g}+B_{1 g}+B_{2 u} .
$$

The $A_{1 g}, E_{g}$ and $B_{1 g}$ modes are Raman active and the $B_{2 u}$ mode is silent. The acoustic modes are $A_{2 u}$ and $E_{u}$ leaving the infrared active modes $5 A_{2 u}+6 E_{u}$. The $5 A_{2 u}$ modes correspond to $z$ or $c$-axis excitations, and the $6 E_{u}$ modes 
to $x, y$ or $a b$ plane phonons. The modes observed in the far-IR optical spectra of the samples presented will display all or most of the modes predicted here by group theory. 


\section{Chapter 3}

\section{Method.}

In measuring the optical constants of the colossal magnetoresistance manganites in the infrared (IR) spectral region, two different techniques were used, namely, normal incidence reflection at Industrial Research Ltd, Wellington, NZ and oblique incidence ellipsometry. The ellipsometry measurements were made at two different places: the Max Planck Insitut für Festkörperforschung, Stuttgart, Germany and the National Synchrotron Light Source at Brookhaven National Laboratory, New York State, USA, in the latter institute taking advantage of the high brilliance IR light source. The normal incidence measurements were performed using two instruments: a FT-IR (Fourier Transform Infrared) system and a grating monochromator. Having laid down the measurement theory of the normal incidence and ellipsometry techniques in the last chapter, this chapter will describe the operation of the spectrometer systems. As all three institutes' spectrometers are FTIR systems, a brief description of Fourier-Transform spectrometry will be given first. Each of the three spectrometers is a complete system comprising sources, beamsplitters, interferometers, detectors and a data processing unit. These configurations of resources will be described for each of the spectrometers in turn. All optical measurements were performed under vacuum, and thus any water vapour, whose vibrational excitations absorb at energies throughout the IR, expunged from the systems. Each system was also attached to an optical bench designed especially for the optical geometries required and these will be separately described, followed by a discussion on the 
facility for making temperature dependent measurements, for each system.

This chapter will also describe the preparation of the single crystal samples that were used. Brief outlines of the method and results of peripheral experiments, such as the magnetisation, x-ray crystal orientation, scanning electron microscope imaging and annealing treatments, will also be described briefly herein.

\subsection{Fourier-Transform Spectroscopy.}

The heart of the FT-IR system is the interferometer, a double armed interference device shown, for the Michelson variety employed in the Nicolet and Bomem systems, as part of the overall spectrometer schematic in Fig. 3.1.

As can be seen in the diagram, the source beam is passed (left) through a beamsplitter (denoted by the dashed diagonal line), which is a material whose dielectric properties are, in the ideal case, such that for certain wavelengths half of the light is transmitted and half reflected. The reflected beam is passed to a fixed mirror $(\mathrm{F})$ at a certain distance from the beamsplitter. The transmitted beam is passed to a moving mirror $(M)$ which moves a distance $\Delta$ either side of a position equal in distance to the fixed mirror from the beamsplitter ${ }^{1}$. Upon reflection from the fixed and moving mirrors the beams are recombined at the beamsplitter which transmits/reflects an output beam to the detector. The output has interference induced by the path difference, $x=2 \Delta$, of the half of the recombined beam that originated from the moving mirror. The modulated output beam from an interferometer is known as the interferogram. The nature of the modulation contains information about the spectral content of the light and allows for the complete spectral decoding of a reflected light signal. The reader is referred to ref. [50] for a complete mathematical description.

\footnotetext{
${ }^{1}$ For the Bomem DA8 spectrometer system the fixed and moving mirror positions are interchanged from Fig. 3.1. It also is unique in its angular setting of the system polarisers, which, without going into detail, allows greater throughput of light. Another variation from this diagram is in the use of a Genzel interferometer for the Bruker 113v system. The major differences are (i) the beam is focused at the beamsplitter, not collimated as in the Michelson, and (ii) the angle of incidence is about $15^{\circ}$, compared with $45^{\circ}$ for the Michelson.
} 


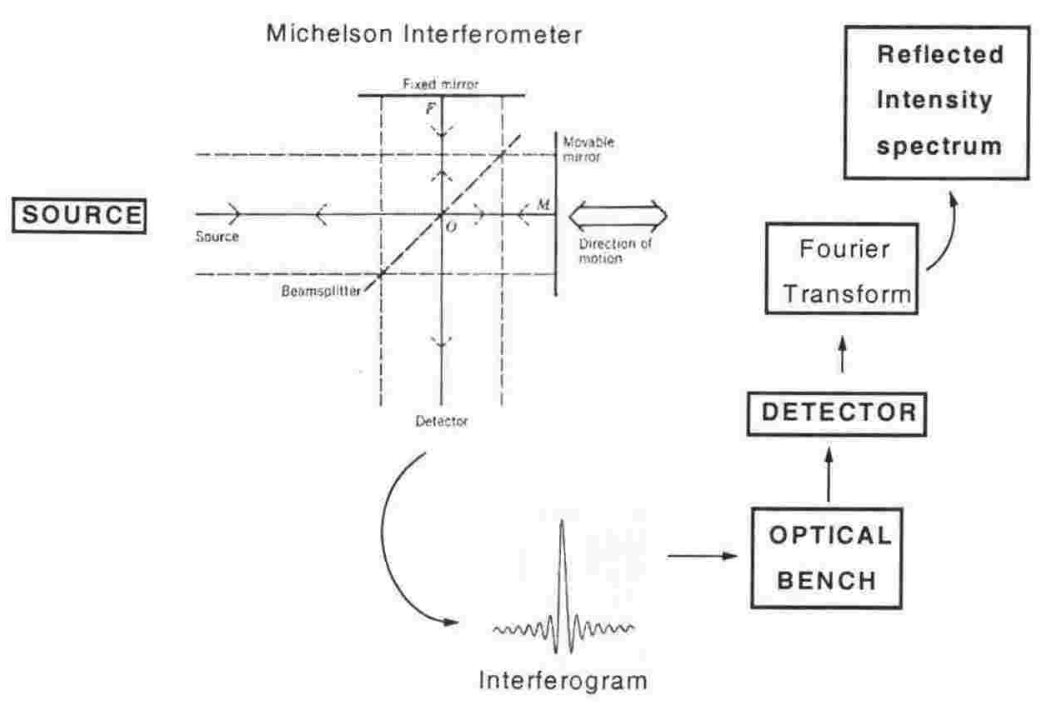

Figure 3.1: Schematic of the Michelson interferometer system in the context of its deployment in the Bomem DA8 and Nicolet Impact 400 spectrometers. Interference produced in the beam by the moving mirror (M) is output as an interferogram, which interacts with the components at the optical bench and is then directed to the detector. The intensity variation with path difference induced by the shifts between moving and fixed mirror positions is Fourier transformed to give the intensity variation as a function of the probing light frequency. Note that focussing and collimating details have been omitted, and displayed is the $45^{\circ}$ beamsplitter schematic only. Reproduced from ref. [50].

The intensity at the detector varies as a function of the path difference between the interferometer arms. The Fourier Transform of the spatial function gives the spectral intensity of the light at the detector as a function of inverted distance units, or wavenumbers $\left(\mathrm{cm}^{-1}\right)$. This encoded light pattern enables spectral information over large frequency ranges to be extracted in seconds. The beamsplitter efficiency, which rarely is that of a perfect beamsplitter with $R=T=0.5$, is not treated here and the detector sensitivity is also ignored. This is because their effects are removed by the ratio measurement detailed in further sections. 


\subsection{Normal Incidence Reflectance.}

The normal incidence reflectivity measurements were made at Industrial Research Ltd, using a Bomem DA8 series FT-IR spectrometer. The spectrometer will be described first, followed by a description of the use of the system for normal incidence reflection measurements.

\subsubsection{Spectrometer resources.}

The wavelengths available to probe our samples are determined by the optical components of the spectrometer, that is, the source, the beamsplitter and the detector configurations. Fig. 3.2 illustrates the frequency-dependent intensity signal at different detectors of the selected source and beamsplitter combinations. Using the resources available enables spectra in the range of $50 \mathrm{~cm}^{-1}$ and $15000 \mathrm{~cm}^{-1}$ to be easily attained. Table 3.1 gives the spectral range of each of the optical components in more detail.

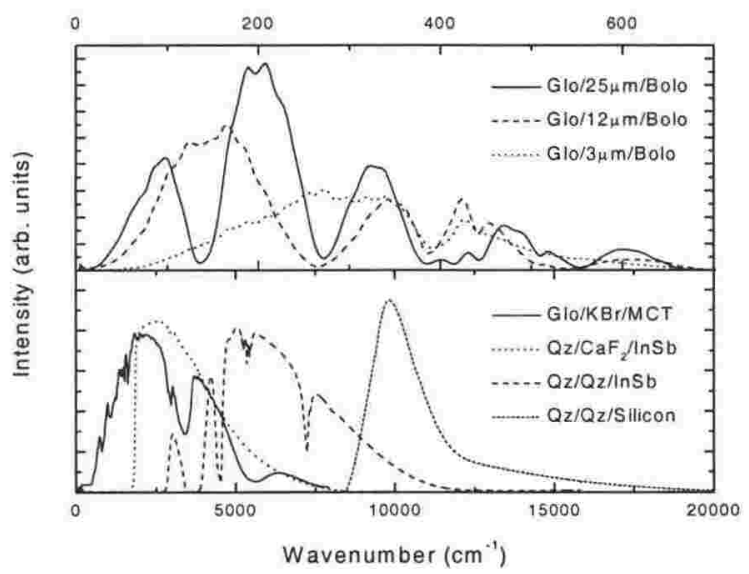

Figure 3.2: Overlap of spectra measured using different combinations of spectrometer resources. Translations of the legend can be found in the Abbreviations column in Table 3.1.

\subsubsection{Optical bench.}

In order to be able to apply the reduced Fresnel equation of 2.44 from the previous chapter, it is necessary that the light should be normally incident 


\begin{tabular}{c||c|c|c}
\multicolumn{1}{c||}{} & Resource & Energies $\left(\mathrm{cm}^{-1}\right)$ & Abbreviation \\
\hline \hline \multirow{4}{*}{ Sources } & Mercury & $5-200$ & $\mathrm{Hg}$ \\
\cline { 2 - 4 } & Globar & $100-10000$ & $\mathrm{Glo}$ \\
\cline { 2 - 4 } & Quartz-Halogen & $2000-25000$ & $\mathrm{Qz}$ \\
\hline \hline \multirow{5}{*}{ Beamsplitters } & $25 \mu \mathrm{m}$ Mylar & $15-100$ & $25 \mu \mathrm{m}$ \\
\cline { 2 - 4 } & $12 \mu \mathrm{m}$ Mylar & $40-200$ & $12 \mu \mathrm{m}$ \\
\cline { 2 - 4 } & $3 \mu \mathrm{m} \mathrm{Mylar}$ & $125-850$ & $3 \mu \mathrm{m}$ \\
\cline { 2 - 4 } & $\mathrm{KBr}$ & $450-4000$ & $\mathrm{KBr}$ \\
\cline { 2 - 4 } & $\mathrm{CaF}$ & $1200-8500$ & $\mathrm{CaF}_{2}$ \\
\cline { 2 - 4 } & Quartz & $4000-25000$ & $\mathrm{Qz}$ \\
\hline \hline \multirow{5}{*}{ Detectors } & He cooled Bolometer & $10-700$ & $\mathrm{Bolo}$ \\
\cline { 2 - 4 } & $\mathrm{N}_{2}$ cooled HgCdTe & $400-5000$ & $\mathrm{MCT}$ \\
\cline { 2 - 4 } & $\mathrm{N}_{2}$ cooled InSb & $1800-14000$ & $\mathrm{InSb}$ \\
\cline { 2 - 4 } & Si & $8500-50000$ & Silicon \\
\cline { 2 - 4 } & & &
\end{tabular}

Table 3.1: Bomem DA8 spectrometer resources and their spectral range.

at the material surface. In practise the beam is incident at as near a normal incident angle as the geometry of the optical components in the sample chamber will allow, or $\theta \approx 12^{\circ}$. The uncertainty in the approximation that $\sin \theta \approx 0$ and $\cos \theta \approx 1$ is figured to contribute to the systematic error of the reflectance measurement. The optical setup for achieving near-normal incidence reflectivity of the spectrometer beam is illustrated in Fig. 3.3.

Light propagating in the vertical plane (by contrast with the Bruker system, in which the light beam is always propagated horizontally) is deflected into the horizontal plane of the sample chamber by a mirror in the interferometer. The first plane mirror in Fig. 3.3 deflects the beam through the aperture to the first focussing mirror. This is a mirror with off axis curvature that focuses the light spot, an image of the aperture, onto the sample. The beam reflected from the sample is focussed onto the detector by a second focussing mirror and a plane mirror at the detector window. The symmetry of the system ensures that the image at the detector is that of the source, thereby allowing maximum collection of the light package with minimal distortion of the contained information. The two plane mirrors and aperture position are only adjustable when the bench is purged, or at atmospheric pressure. Fine tuning of the beam path can be achieved when the optical 


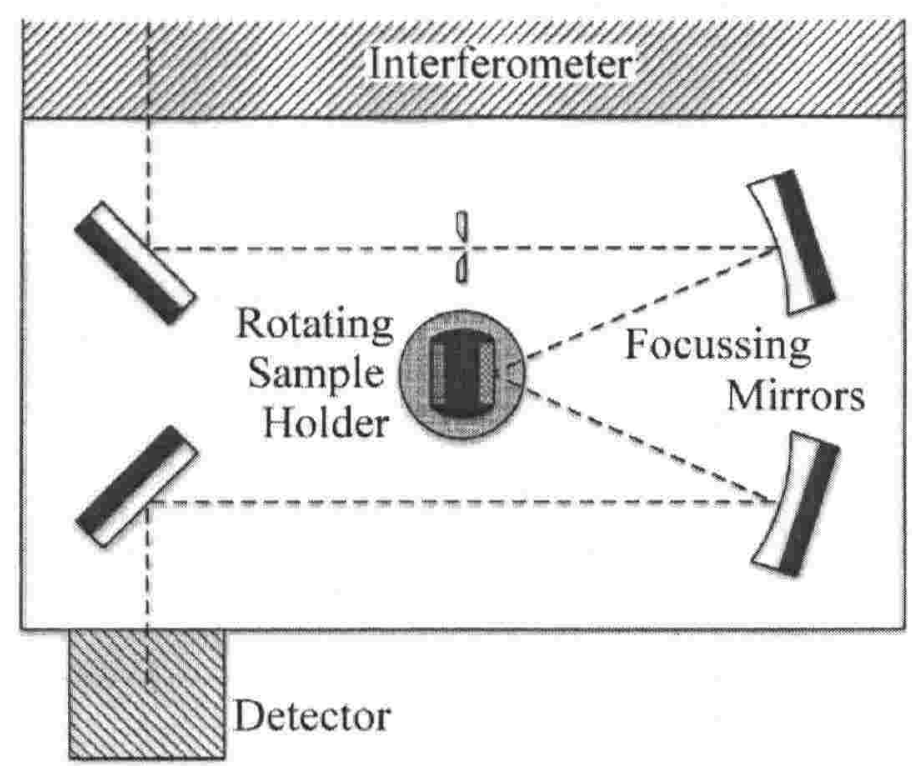

Figure 3.3: Representation of the Industrial Research Ltd designed optical bench, attached to the DA8 series Bomem spectrometer, and used for normal incidence reflection. Diagram courtesy of [51].

bench is under vacuum however, using servo controlled motors on the adjustment pins of the two off-axis mirrors.

\subsubsection{From transmission to reflection.}

The Fourier-transformed measurement at the detector is of the frequencydependent intensity variation of the light reflected from the sample. The reflectance spectrum at this juncture is the transmission spectrum of the combined source, beamsplitter, sample and detector configuration, as can be seen for different combinations of source, beamsplitter and detector in Fig. 3.2. However the reflectivity of the sample material cannot be extracted from just this measurement. In order to find the absolute reflectivity of a particular sample, we must compare the sample reflectance to the measured reflectance of a material whose absolute reflectivity is known. Useful materials for which this is so include aluminium and gold, which both have very high and wellknown IR reflectivities, are relatively stable and show limited sensitivity to 
oxidation. For use as a reference gold films were evaporated onto optically flat glass plates which were first overcoated by sputtering with a chrome polish. Gold reflects in the order of $99 \%$ of IR radiation incident upon it[52], and all spectra are corrected for this reported reflectivity of gold. The difference between evaporated gold and literature gold reflectivities, is recognised as another contribution to the systematic error of the reflectance measurement.

The so-called "gold edge", or decline in the reflectivity of gold caused by interference due to interband absorptions, is in the region of visible light, so for energies greater than about $20000 \mathrm{~cm}^{-1}$ gold begins to expend its usefulness as a reference. Also, at these energies the size of the gold particles evaporated onto the film become comparable to the wavelength of the light being used to probe the materials. This leads to an increased scattering of shorter wavelength light, and the gold referencing technique is thus less reliable at higher frequencies of incident light.

For reflection measurements in the IR, a rotating sample holder was used with sample and reference mounted back to back. The holder was then rotated through $180^{\circ}$ to enable the placement of either the sample or the reference in the path of the spectrometer beam. To ensure that the reflected light from both the sample or the reference followed 'exactly' the same optical path to the detector, a laser beam, independent of the spectrometer, was focused on each surface. The laser source was positioned approximately 1 $\mathrm{m}$ from the sample and reference surfaces, thereby producing a combined incident-reflected beam length of the order of $2 \mathrm{~m}$. The positions of the reflected laser spots were monitored on a white screen at the laser source and made to coincide to within $1 \mathrm{~mm}$ upon rotation of the holder. This precision corresponds to the beams from the sample and reference coinciding to within at least $1 \mathrm{~mm}$ of each other on the detector. This is important, in order to avoid uncertainties due to spatial differences of the detector material.

The sample holder sits on the end of a rod which spans the approximately $1 \mathrm{~m}$ height of the bath cryostat used for temperature-dependent measurements. Because of this length, any thermal contraction of the rod is exaggerated and must be accounted for in the optical set-up. Typically a shift in position of the sample and reference of $4 \mathrm{~mm}$ is found for a decrease 

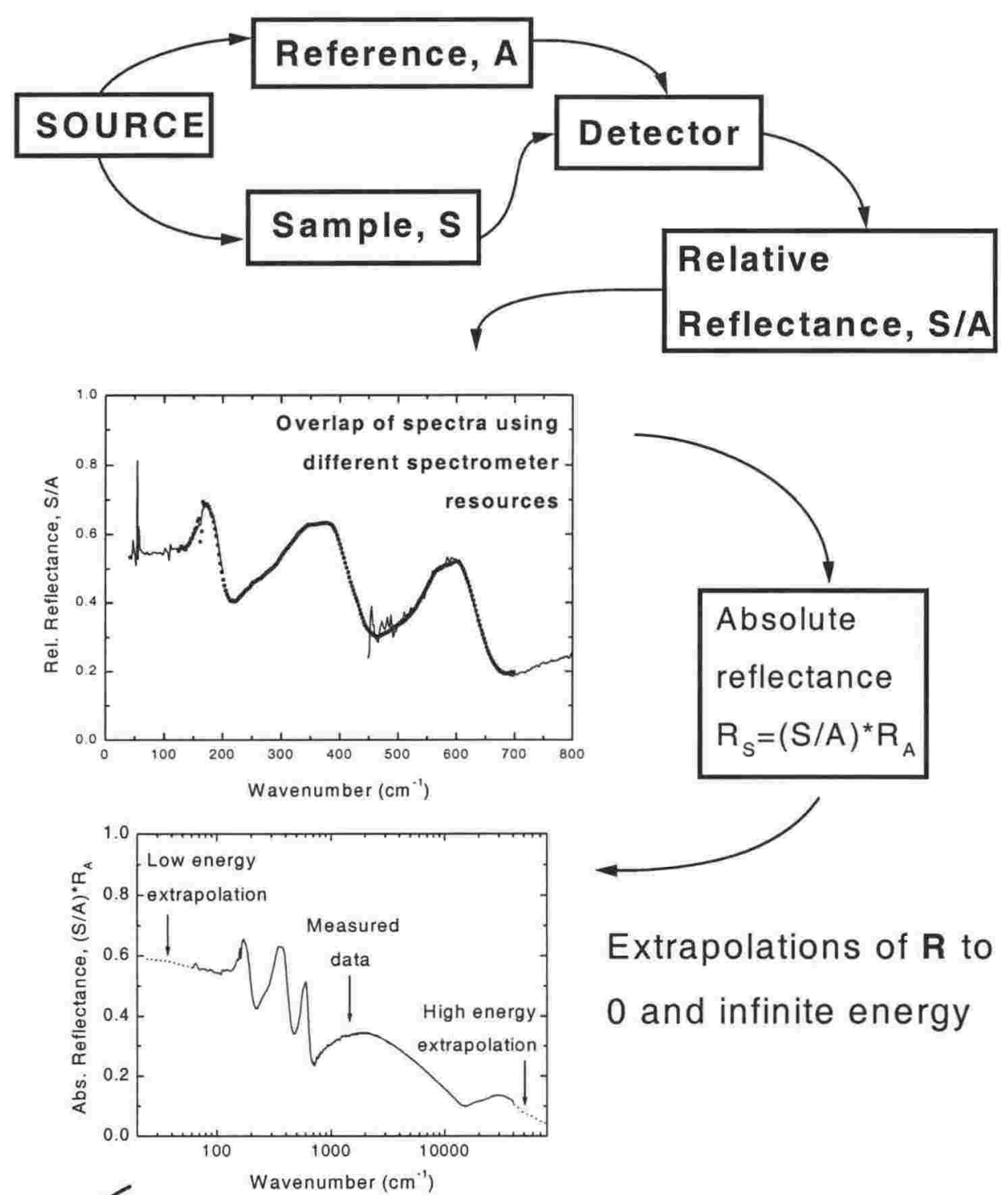

Extrapolations of $\mathbf{R}$ to

0 and infinite energy

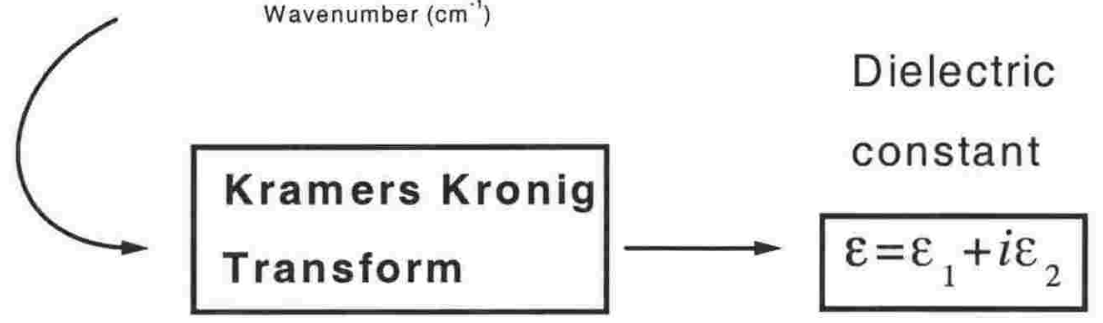

Figure 3.4: Schematic of the measurement and data reduction process for Normal Incidence Reflectivity. 
in temperature from $295 \mathrm{~K}$ to $77 \mathrm{~K}$. To counteract this shifting of position, at every temperature at which measurements were made, the spectrometer and alignment laser beams were refocused onto the sample and reference. Then the optical bench was realigned and the output signal optimized before the measurement was made.

Thus, in a typical measurement run, as seen in Fig. 3.4, intensity measurements of the sample and reference are made by the detector, then ratioed to give $\mathrm{S} / \mathrm{A}$, the relative reflectance. This is done in a certain spectral range, depending on the spectral resources chosen. The process is repeated using other spectrometer resources until the desired energy range is covered. If all optical elements have been optimally aligned, the overlap between spectra will be good, as seen for three different spectral ranges in the top diagram of Fig. 3.4. At this juncture the various relative reflectance spectra are spliced together and the absolute reflectance (denoted $R_{S}$ ) found by taking the reflectance of the reference material $\left(R_{A}\right)$ into account. High and low energy extrapolations are then applied to the absolute reflectivity spectrum (bottom diagram of Fig. 3.4), in order to meet the Kramers-Kronig transform requirement that $R$ be known to all frequencies. The real and imaginary parts of the dielectric constant are then extracted using the transform.

To illustrate the reproducibility of the combination spectrometer, optical bench and sample holder arrangement, Fig. 3.5 shows four spectra measured, from one of the single crystals in this report, in the space of 10 minutes and with scan times of 20 seconds. They are the reflectance of the material with respect to gold, or $R_{s}$. It can be seen that the maximum deviation (ignoring the noisy peaks below $200 \mathrm{~cm}^{-1}$ ) from the eyeball average of the four spectra is at around $580 \mathrm{~cm}^{-1}$, and is no more than $2 \%$, or less than $4 \%$ of the signal magnitude. The scan reproducibility and sample repositioning error in $R$ is much less than this on the average, at about $\pm 1 \%$. The uncertainty in $R$ then, due to the entire spectrometer system and optical bench set-up, also including the effects of the reference, is taken as $\pm 3 \%$. 


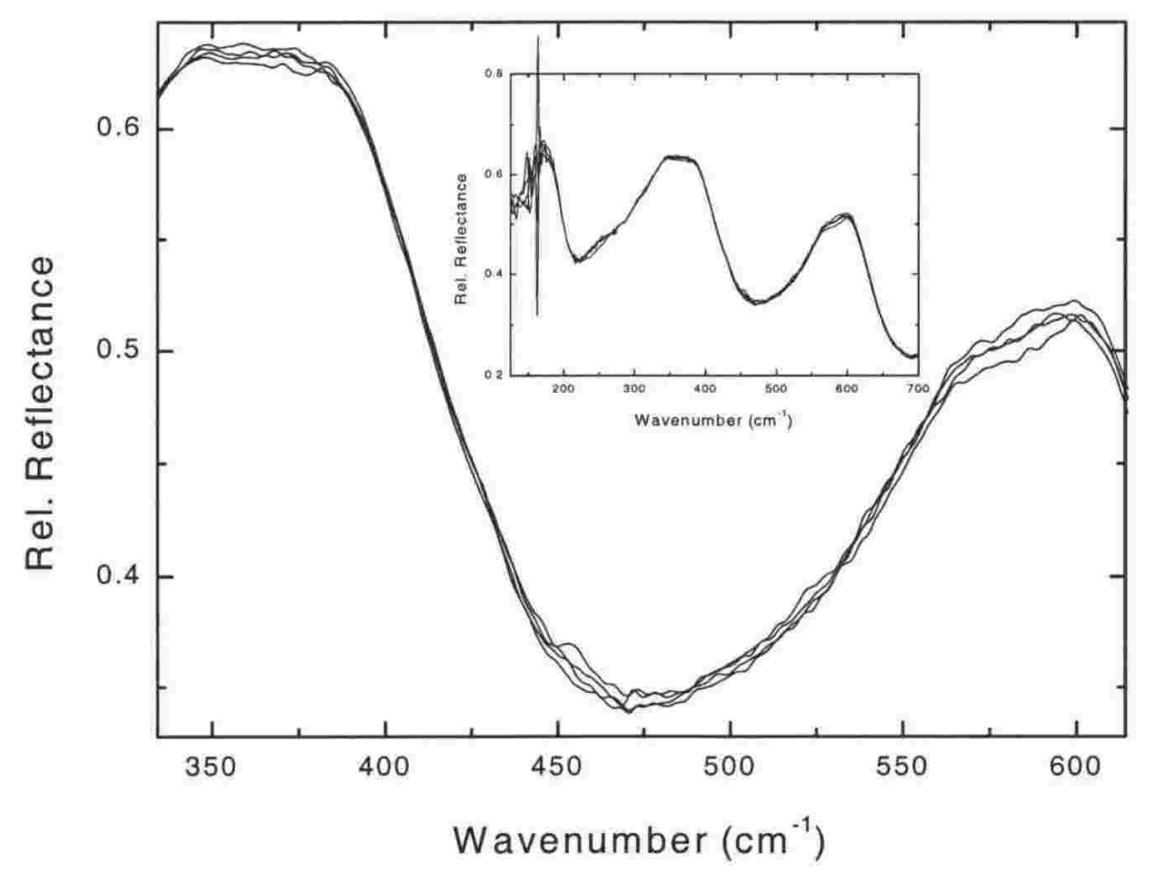

Figure 3.5: 4 spectra demonstrating the reproducibility of the Bomem DA8 Spectrometer, optical bench and sample holder arrangement.

\subsubsection{Temperature-dependent measurements.}

An MD4 Oxford Bath cryostat was used for low temperature normal incidence reflection measurements in the IR. This is designed to hold either liquid nitrogen or liquid helium as cryogen, but for the present experiment liquid nitrogen was used. The optical tail which carries the sample, makes thermal contact with the cryogen via a heat exchange space in which helium gas was used. The tail was also availed of a heating element controlled by a Lakeshore 330 Autotuning temperature controller.

\subsubsection{Grating Monochromator.}

The normal incidence reflectance measurements were extended into the region of visible light $\left(10000 \mathrm{~cm}^{-1}-50000 \mathrm{~cm}^{-1}\right)$ by using a prism and grating monochromator spectrometer maintained by the Light Standards team at 
Industrial Research Ltd. This selects different wavelengths in order to probe the sample with monochromatic light, in contrast to the Fourier transform method. The reflected intensity from a surface was measured for each wavelength, and the ambient radiation accounted for with background intensity measurements taken before and after each measurement.

\section{Optical bench and resources}

In the visible spectral region the size of the particles of gold on evaporated films becomes comparable to the wavelength of probing light, so we preferentially adopt a method developed by the Light Standards team at Industrial Research Limited and use quartz as our reference. The refractive index of quartz has been extremely well characterised[53] and the reflectance calculated from this is about $4 \%$ in the visible region. To ensure that there are no multiple reflections from the back surface of the quartz plate (thereby giving a false reflected intensity measurement), the back surface of the quartz was roughened to scatter any stray light due to internal reflections. It is possible to also use wedged crystals to scatter light reflected from the back surface, but this requires a very precise geometric setup of the sample to ensure that the reflected beams from both reference and sample follow the same optical path to the detector. The incident angle was in practise $\theta \approx 12^{\circ}$, or the same as that of the Bomem optical bench.

A silicon detector was carefully positioned at the focal point of the reflected image from the reference and sample, using visible light at a wavelength of $550 \mathrm{~nm}$.

The source used was a high brilliance quartz-halogen lamp with a broad spectral characteristic in the visible region[53]. A slit width of $2 \mathrm{~mm}$ was chosen, which acted as the virtual object to the rest of the spectrometer system.

\section{Temperature-dependent measurements}

An Oxford flow-through cryostat with sapphire windows was used for normal incidence reflection measurements in the visible region and liquid nitrogen 
used as the coolant, to obtain sample temperatures down to $80 \mathrm{~K}$.

The sample and the reference were affixed to the cryostat sample holder one above the other and manually moved into the beam position between runs. This necessitated careful checks that horizontal plane rotation had not shifted the beam off the detector.

\subsection{Ellipsometry.}

As previously mentioned, the ellipsometry measurements were made in two different laboratories. A Bruker $113 \mathrm{v}$ spectrometer was used at the the Max Planck Institut in Stuttgart, and a Nicolet Impact 400 vacuum Fourier spectrometer used at the U4IR beamline at the National Synchrotron Light Source on Long Island. The resources and components of each of these different types of spectrometer are very similar. The only significant differences between spectrometers are in the optical bench and light sources used, so these will be described seperately for the Brookhaven experiment in the next section.

The technique of ellipsometry has two advantages over the normal incidence reflectivity technique. One is that determination of the absolute reflectivity of the sample is not required. This eradicates the need, firstly, for reference materials, mandatory in normal incidence reflectivity measurements. Secondly, it dispenses with the need for special sample holders to ensure accurate copositioning of sample and reference. Two significant sources of uncertainty, first, how well known is the reflectivity of the evaporated gold film, and second, how accurately the sample and reference beam paths can be made to coincide, are thus eliminated. The other advantage of ellipsometry over normal incidence reflectivity is that both the real and imaginary parts of the optical constants are determined. The ellipsometry experiment gives information on the reflectivity AND the phase change of the reflected light. By contrast, the normal incidence reflectance measurement's single quantity, the reflectivity magnitude, necessitates extrapolations beyond the regions of acquired data to apply dispersion relations. The elimination of the extrapolation requirement for the ellipsometry technique is another source of 

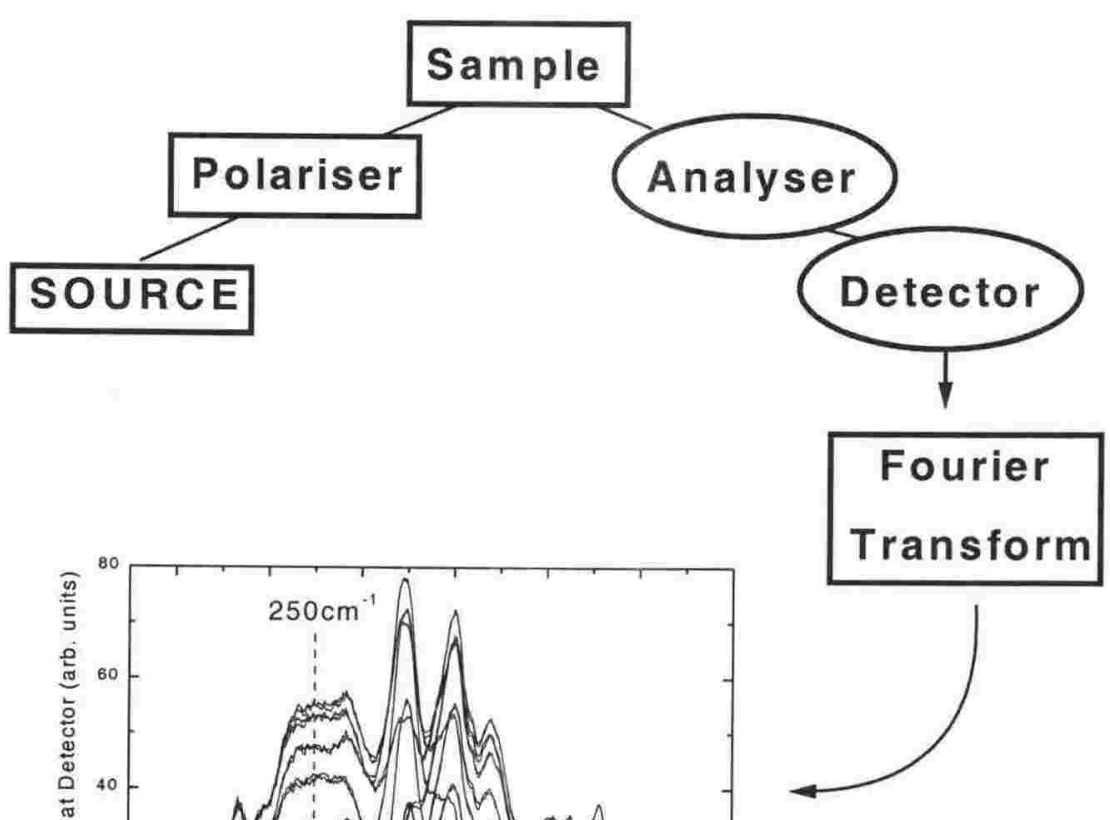

spectra at different

Analyser angles

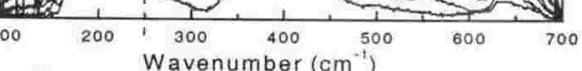

Wavenumber $\left(\mathrm{cm}^{-1}\right)$

at each frequency

Inversion of

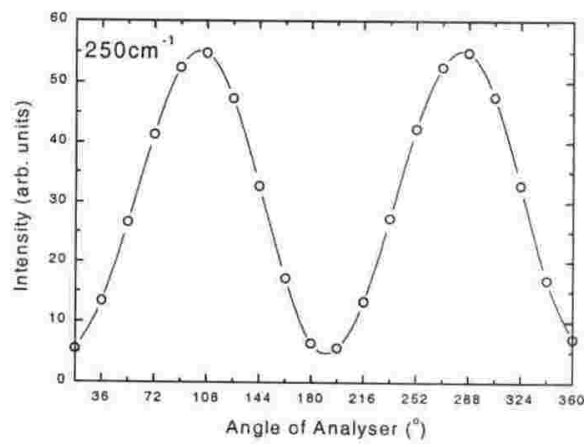

Fresnel

equations

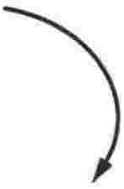

$\varepsilon=\varepsilon_{1}+i \varepsilon_{2}$

Figure 3.6: Schematic of the measurement and data reduction process for ellipsometry. 
reduced uncertainty.

However, ellipsometry has significant disadvantages of its own. Light loss due to scattering from rough surfaces is more pronounced for high incident angle reflection (necessary, as detailed in the next paragraph), especially from small samples. Diffraction effects at the long wavelengths of the farIR can also skew the ellipsometry measurements. Thus typical ellipsometry measurements routinely demand either large samples or very high brilliance light sources. Also, as will be appreciated by the data acquired on the layered manganites, when anisotropic samples are measured by ellipsometry, normal incidence measurements often must serve as a guide to help reduce the data.

Fig. 3.6 is a schematic of the measurement and data acquisition process in the ellipsometry technique. The five boxes making the shape of an arrowhead at the top of the diagram were introduced in the previous chapter. In review, the light from the source is linearly polarised at the sample by the Polariser, set at a fixed angle $P$. The angle of incidence of the light is chosen to be near the Brewster angle of the material, so that the difference between the $r_{p}$ and $r_{s}$ components of the light is at a maximum. The sample reflects the obliquely incident light so that it is elliptically polarised and passes this light to the detector via another polariser. This second polariser (or Analyser) rotates through $360^{\circ}$, allowing the detector to make intensity measurements at defined positions, in this case every $18^{\circ}$. The intensity as a function of interferometer path difference is Fourier transformed for each analyser angle $A$, to give the 20 intensity spectra pictured in the top most graph. At each frequency the angular variation in the intensity follows a sinusoidal curve which can be fitted to equation 2.31. Then, using the theory developed in the previous chapter, the Fresnel equations, 2.14 and 2.15, may be inverted at each frequency to extract the dielectric constant.

\subsubsection{Spectrometer Resources.}

The spectrometer resources are very similar to those used in the Bomem system. They are briefly detailed in Table 3.2. An identical far-IR bolometer detector, is adapted for the detection of mid-IR radiation by the fitting of a 
cold Ge filter, and is operated at $4 \mathrm{~K}$. The far-IR bolometer is operated at $1.2 \mathrm{~K}$ by pumping on the helium liquid in the bolometer housing cryochamber.

\begin{tabular}{c||c|c|} 
& Energy Range & Resources used \\
\hline \hline \multirow{3}{*}{ Bruker 113v } & $50-500 \mathrm{~cm}^{-1}$ & $6 \mu \mathrm{m}$ Mylar, Hg, far-IR Bolo \\
\cline { 2 - 3 } & $150-700 \mathrm{~cm}^{-1}$ & $3 \mu \mathrm{m} \mathrm{Mylar,} \mathrm{Hg,} \mathrm{far-IR} \mathrm{Bolo}$ \\
\cline { 2 - 3 } & $400-6000 \mathrm{~cm}^{-1}$ & $\mathrm{KBr}$, globar, mid-IR Bolo \\
\hline \hline \multirow{2}{*}{ Nicolet 400 } & $50-700 \mathrm{~cm}^{-1}$ & Si solid substrate, SLS, far-IR Bolo \\
\cline { 2 - 3 } & $500-3000 \mathrm{~cm}^{-1}$ & Si solid substrate, SLS, mid-IR Bolo \\
\cline { 2 - 3 }
\end{tabular}

Table 3.2: Spectral coverage of the Bruker 113v and Nicolet 400 spectrometers with resource combinations required for each range. The abbreviations are the same used in Table 3.1 with SLS denoting the Synchrotron Light Source used at Brookhaven.

Black polythene filters are used when the mercury $(\mathrm{Hg})$ sources are employed to protect the windows and other plastic components from UV radiation in the $\mathrm{Hg}$ source.

\subsubsection{Optical bench.}

The ellipsometry setup used at the Max Planck Institut can be discussed from the schematic in Fig. 3.7. The FT-IR part of the system in the middle of the diagram passes the incident beam to a pre-chamber, where it is reflected to the ellipsometer chamber by the plane mirror (M1). The light is polarised on passing through the polariser, $(\mathrm{P})$, set at a particular fixed angle for an experimental run. The light then passes through the cryostat (L) window to the sample $(\mathrm{S})$, from whence it is reflected through the second cryostat window elliptically polarised. The rotating analyser (A) then passes the beam from the collecting mirror (M2) to the detector (Bo), where the intensity of the beam is recorded.

\subsubsection{Sample mounting.}

The samples are mounted with GE varnish (an adhesive that has a low thermal expansivity) to brass pins, which are then oriented manually so that the sample lies in the path of the incoming beam. The required angle of 
incidence is obtained by rotating the sample mount to bring the reflected light from the sample onto a predesignated position, marked on the inside wall of the optical bench. The opening angle, or the spread of the light from its nominal angle of incidence for the system, is $1.2^{\circ}$. A calculation detailed in Kircher and coworkers[54], found an uncertainty in the optical constants of the order of $\pm 2 \%$ as a result of this angular spread.

\subsubsection{Temperature-dependent measurements.}

Temperature-dependent ellipsometry measurements were made with the sample housed in a helium flow-through cryostat with wedged diamond windows. The same cryostats were transported from the Max Planck Institut for use at Brookhaven. The windows on the cryostat are positioned such that they admit and transmit light at predefined oblique incident angles. To this end two different cryostat casings were used for the two different incident angles of $\theta=75^{\circ}$ and $\theta=80^{\circ}$.

\subsection{Ellipsometry at Brookhaven}

\subsubsection{Optical bench.}

The setup used at Brookhaven is pictured in Fig. 3.7. The U4IR working area at the National Synchrotron Light Source is situated on a platform some metres above the level at which radiation is ejected from the synchrotron radiation ring. The U4IR beam, then, is deflected vertically from this level to the working level and passed through a wedged diamond window (DW) via a configuration of plane mirrors. After passing through the interferometer compartment with the beamsplitter and moving mirror (denoted St and B respectively) the beam is passed into a pre-chamber where a compensator is often employed at D. The ellipsometer chamber is then as discussed in section 3.3.2. 


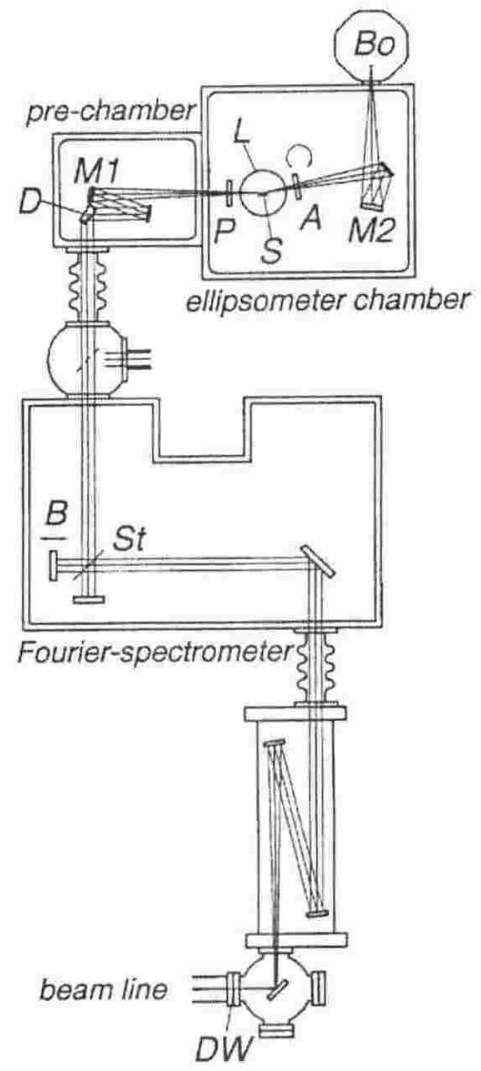

Figure 3.7: Brief schematic of the essential components in the ellipsometry setup used at the National Synchrotron Light Source at Brookhaven National Laboratory. Diagram from Henn[55].

\subsubsection{Beamline characteristics}

The spectral characteristic of the U4IR beamline is shown in Fig. 3.8 (upper curve) in comparison with a typical globar source emitting at $2000 \mathrm{~K}$ [53]. The intensity of the synchrotron light source is a remarkable 2-3 orders of magnitude greater than the globar ${ }^{2}$ across the spectral range of $10-10000 \mathrm{~cm}^{-1}$. This enables the measurement by ellipsometry of much smaller samples than a regular source will allow, and over a greater spectral range.

\footnotetext{
${ }^{2}$ In this wavelength range the globar source actually produces more photons over $4 \pi$. The information displayed, thus, is only true for very small solid angles, which we have in the instance of this experiment.
} 


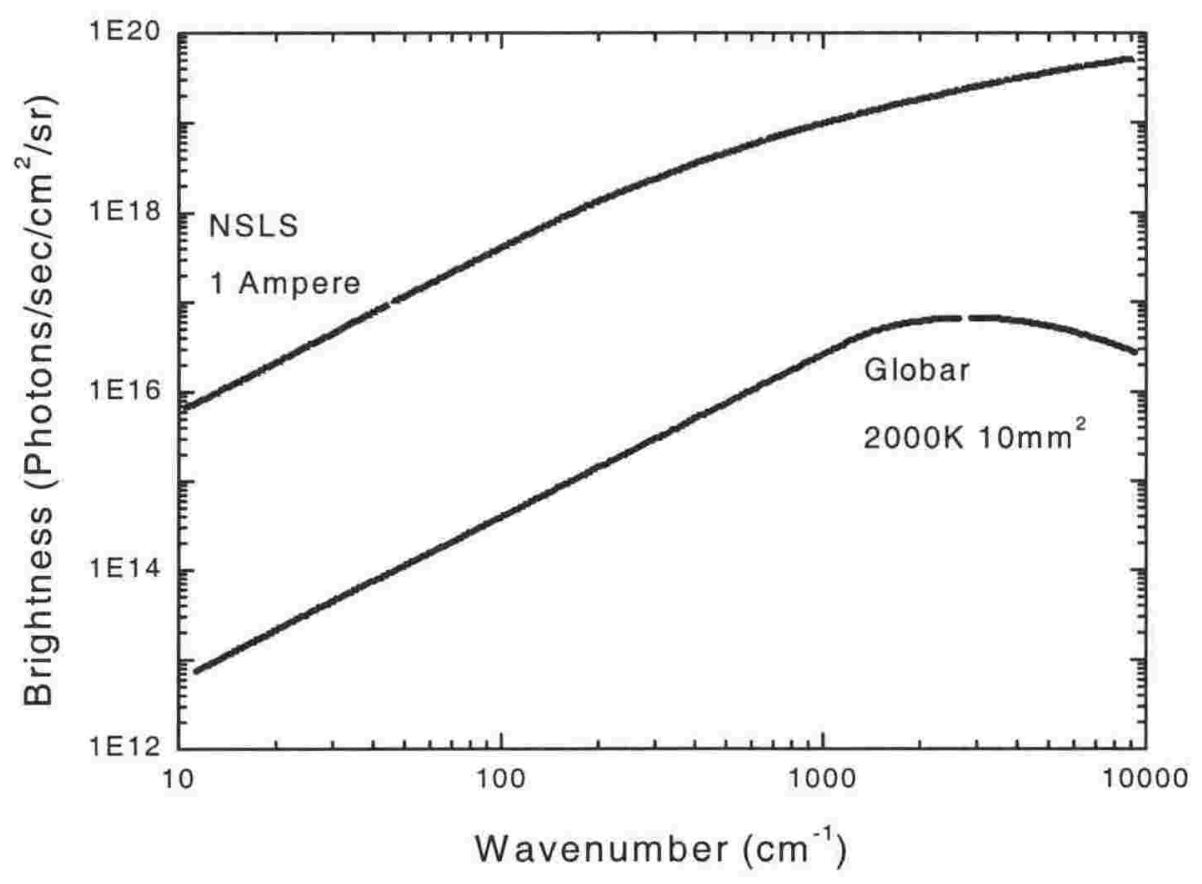

Figure 3.8: U4IR beamline spectral characteristic at the National Synchrotron Light Source at Brookhaven National Laboratory from [56].

\subsection{Sample Preparation.}

\subsubsection{Preparing surfaces for measurement.}

It is essential that, for the material whose optical properties we are trying to measure, the reflecting surface is smooth on the scale of the wavelength of light being used to probe the sample, so that the reflection is specular. If some of the reflected light is lost by scattering, it causes an underestimation of $R$ and thus alters the conductivity extracted from the Kramers-Kronig transform. For samples which do not have a naturally reflective surface, specular reflection is usually achieved by polishing with progressively finer grains, diamond paste and $\mathrm{Al}_{2} \mathrm{O}_{3}$ powder being common choices of abrasive. However, as will be detailed in the next chapter, polishing creates a damaged layer, possibly by straining the crystal, even to the extent of changing 


\begin{tabular}{|c|c|c|c|c|c|}
\hline Sample & $x$ & Surface & Treatment & Anneal. Time & Temp \\
\hline \multirow{2}{*}{$\mathrm{La}_{1-x} \mathrm{Ca}_{x} \mathrm{MnO}_{3}$} & 0.1 & polished & annealed & 1 hour & $1000^{\circ} \mathrm{C}$ \\
\hline & 0.265 & polished & annealed & Hours & $650^{\circ} \mathrm{C}$ \\
\hline \multirow{2}{*}{$\begin{array}{r}\mathrm{La}_{2-2 x} \mathrm{Sr}_{1+2 x} \mathrm{Mn}_{2} \mathrm{O}_{7} \\
a b \text { plane } \\
c \text {-axis } \\
\end{array}$} & \multirow[t]{2}{*}{0.4} & cleaved & - & - & - \\
\hline & & polished & - & - & - \\
\hline \multirow{2}{*}{$\begin{array}{r}\mathrm{Pr}_{2-2 x} \mathrm{Sr}_{1+2 x} \mathrm{Mn}_{2} \mathrm{O}_{7} \\
a b \text { plane } \\
c \text {-axis }\end{array}$} & \multirow[t]{2}{*}{0.5} & cleaved & - & - & - \\
\hline & & polished & - & - & - \\
\hline
\end{tabular}

Table 3.3: Surface treatment parameters for manganite samples.

the measured reflectivity, so one must be careful to anneal the sample after polishing. The thermal treatment corrects some of the damage induced by polishing, but introduces other problems, which will be discussed in later sections.

For the bilayered manganites, the physical coupling strength between the planes of $\mathrm{MnO}_{6}$ octahedra is quite weak, and the samples thus cleave very easily along the $a b$ planes. For the single crystals, this produces very flat and mirror-like surfaces, so that polishing of the $a b$ plane is not required. The $c$-axis plane of each sample however, is polished to be specularly reflecting.

A summary of the samples measured in this study and their surface preparation treatments is presented in Table 3.3 .

\subsubsection{Pseudocubic $\mathrm{La}_{1-x} \mathrm{Ca}_{x} \mathrm{MnO}_{3}$.}

Both $\mathrm{La}_{0.9} \mathrm{Ca}_{0.1} \mathrm{MnO}_{3}$ and $\mathrm{La}_{0.735} \mathrm{Ca}_{0.265} \mathrm{MnO}_{3}$ are single crystals grown by the floating zone method. This fabrication method was carried out by a Japanese group at Joint Research Center for Atom Technology in Tokyo and the details are available elsewhere[25]. High quality single crystals, in the form of cylindrical rods, of a typical length of $50 \mathrm{~mm}$ are produced by the process, and the samples themselves are cut lengths of about $5 \mathrm{~mm}$. The samples are hard and black in appearance. 


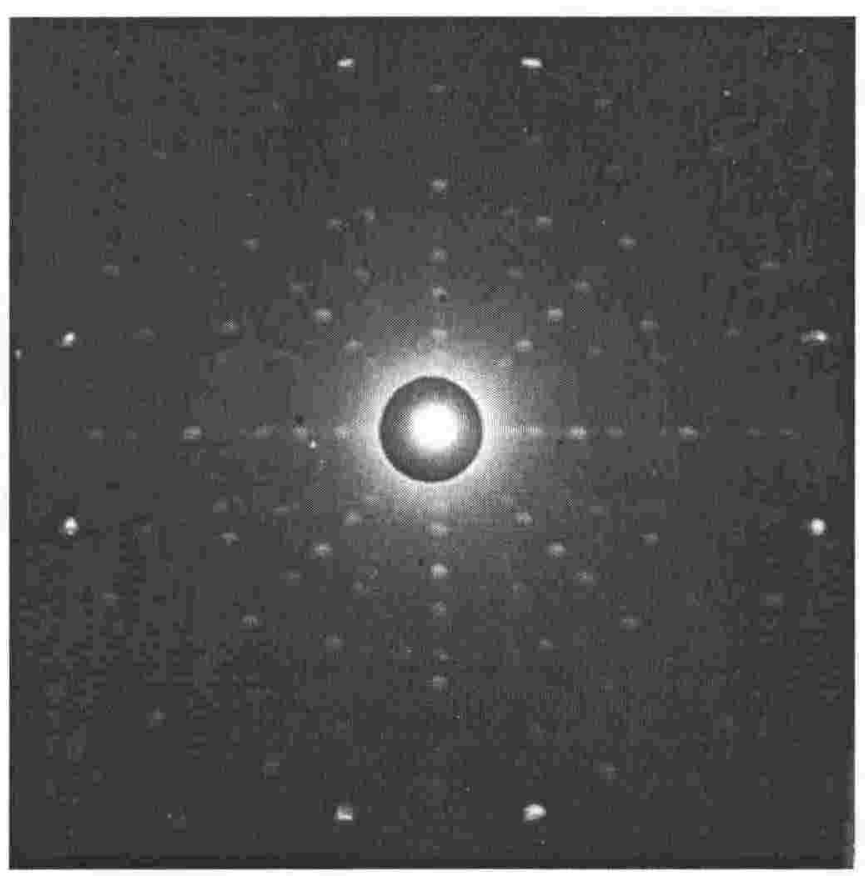

Figure 3.9: Laue x-ray diffraction pattern of the $\left(\begin{array}{lll}1 & 0 & 0\end{array}\right)$ face of $\mathrm{La}_{0.735} \mathrm{Ca}_{0.265} \mathrm{MnO}_{3}$.

\section{Crystal orientation by Laue diffraction}

To assist in locating the $\left(\begin{array}{lll}1 & 0 & 0\end{array}\right)$ face of the sample, in order to polish for reflection measurements, the technique of Laue x-ray reflection was used. This method allows, amongst other things not detailed here, the determination of the high axes of symmetry of a single crystal sample. Briefly, x-rays with a range of wavelengths are directed at a crystal and reflected back onto a photographic plate at the source. Bragg reflection, or, loosely speaking, constructive interference, occurs if the range of wavevectors coincides with the distance between planes of atoms in the crystal. The author found [57] useful as a reference.

The sample itself was mounted on a goniometer, allowing free rotation of the sample in the orientation adjustment between exposures. An initial photo taken for a random orientation of the sample gave a non-symmetrical pattern, with the $\left(\begin{array}{lll}1 & 0 & 0\end{array}\right)$ axis of symmetry off-centre. Subsequent adjustments to the sample position and further photographs enabled a gradual orientation 
of the $\left(\begin{array}{lll}1 & 0 & 0\end{array}\right)$ plane to face the source. Displayed in Fig. 3.9 is a selected photograph taken with 15 minutes exposure time of the crystal to the x-rays, with a $\left(\begin{array}{lll}1 & 0 & 0\end{array}\right)$ face squarely facing the $\mathrm{x}$-ray source. The hole and bright spot in the centre are, respectively, the hole in the photographic plate which admits the x-ray source, and the source itself. The spots on the image are reflections corresponding to the crystal's planes in reciprocal space. The fourfold symmetry evidenced by various features on the photo - the four compass point arms and the bright pairs of spots at the edges of the image, for example - is indicative of $\left(\begin{array}{lll}1 & 0 & 0\end{array}\right)$ symmetry.

\section{Polishing particulars}

We chose a flat sawn face in the $\left(\begin{array}{lll}1 & 0 & 0\end{array}\right)$ plane of each of samples, and wet polished them to gain a specularly reflecting surface. Because of the importance of a flat reflective surface to the normal incidence measurement, the sample to be polished was mounted in a fashion developed by Dave Cochrane[58]. The sample was mounted onto a glass holder with wax, and surrounded by a material of similar hardness (glass was deemed appropriate) to approximately the same height as the sample. The glass surrounding the sample then acted as a supporting brace, and ensured that the sample face was polished flat. Without this support, the strains on the sample cause it to move under the physical strain of the polishing action, and tend to polish up a convex surface. Progressively finer polishing grains of sandpaper were used. The sample face was polished off with $1 \mu \mathrm{m} \mathrm{Al}_{2} \mathrm{O}_{3}$ powder on a rotating pitch plate moistened with distilled water. This gave a mirror-like surface from which the reflectance was measured.

\section{Annealing particulars}

To release the strain that polishing induces near the surface of the single crystals, an annealing treatment was used. A significant portion of the results section will discuss the differences between spectra measured from the polished surface of the materials as opposed to the polished and then annealed material. Put forth here is the "what" of the procedures used, leaving 


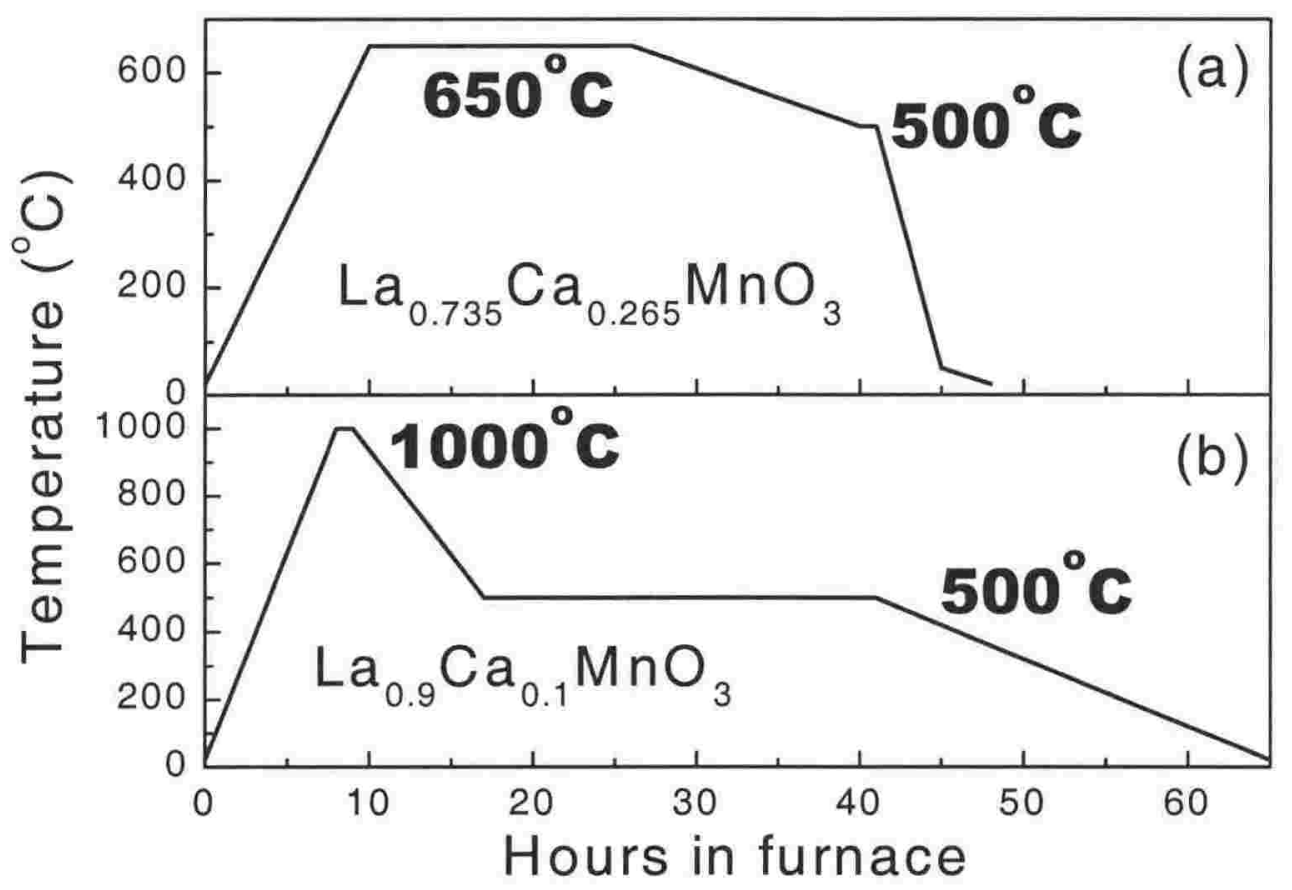

Figure 3.10: Furnace profiles for the annealing treatments given to the (a) $\mathrm{La}_{0.735} \mathrm{Ca}_{0.265} \mathrm{MnO}_{3}$ and (b) $\mathrm{La}_{0.9} \mathrm{Ca}_{0.1} \mathrm{MnO}_{3}$ samples.

the "why" to the next chapter. As seen in Fig. 3.10(b), which displays the furnace profiles, the $x=0.1$ sample was annealed for 1 hour in a flowing 100 $\%{ }^{16} \mathrm{O}_{2}$ atmosphere, at $1000^{\circ} \mathrm{C}$, following the procedure adopted by Lee and coworkers[32]. Optical measurements were performed on a crack-free region of the surface with a surface area of $\pi(1.5 \mathrm{~mm})^{2}$. The $x=0.265$ sample was annealed at the lower temperature of $650-500^{\circ} \mathrm{C}$ for about 30 hours, also in a flowing ${ }^{16} \mathrm{O}_{2}$ atmosphere as illustrated in Fig. 3.10(a). This annealing treatment matted the surface of the $x=0.265$ sample somewhat, but no cracking was seen in this material, which also presented a surface area to the beam of $\pi(1.5 \mathrm{~mm})^{2}$. 


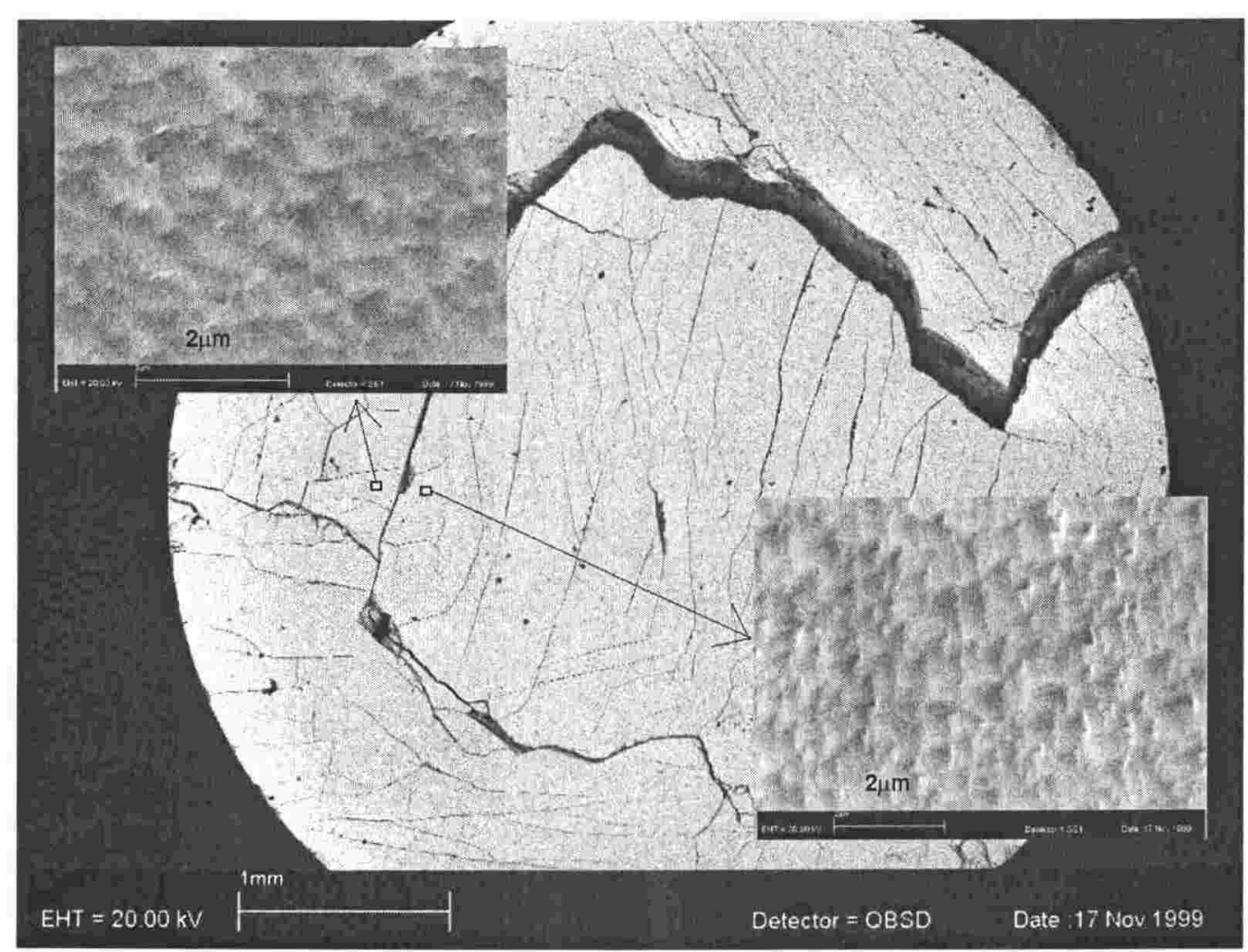

Figure 3.11: Scanning Electron Microscope image of the surface of the $\mathrm{La}_{0.9} \mathrm{Ca}_{0.1} \mathrm{MnO}_{3}$ single crystal used for optical reflectivity. The insets show close ups of the indicated regions of the surface. Note that boxes indicating the blown up regions are NOT scale representatives of the inset boxes.

\section{Scanning Electron Microscope images}

The Scanning Electron Microscope (SEM) at Industrial Research Ltd was used to image the surface of $\mathrm{La}_{0.9} \mathrm{Ca}_{0.1} \mathrm{MnO}_{3}$ at different magnifications, and a composite image of some of these is displayed in Fig. 3.11. As can be seen in the main image, there are at least two large cracks on the $\mathrm{La}_{0.9} \mathrm{Ca}_{0.1} \mathrm{MnO}_{3}$ sample surface, and a great number of small cracks, secondary-fault-like, across the entire sample face. The region of the sample used for optical measurements was a central circle of approximately $3 \mathrm{~mm}$ in diameter, some of the boundary of which was defined by the largest crack. The large cracks shown on this SEM image were apparent before annealing, but were about a quarter of the width, which may indicate that the thermal treatment caused 
the release of strains in the material. The two insets to Fig. 3.11 display blown up regions of parts of the surface. The rightmost inset displays a surface with ridges having a periodicity of about $400 \mathrm{~nm}$. The leftmost inset displays a surface with ridges having a periodicity of about $600 \mathrm{~nm}$ and the preferential direction of the ridges appears to have rotated $90^{\circ}$. Nonetheless, both of these roughnesses are fairly smooth on the scale of the wavelength of IR radiation.

\section{Magnetisation measurements}

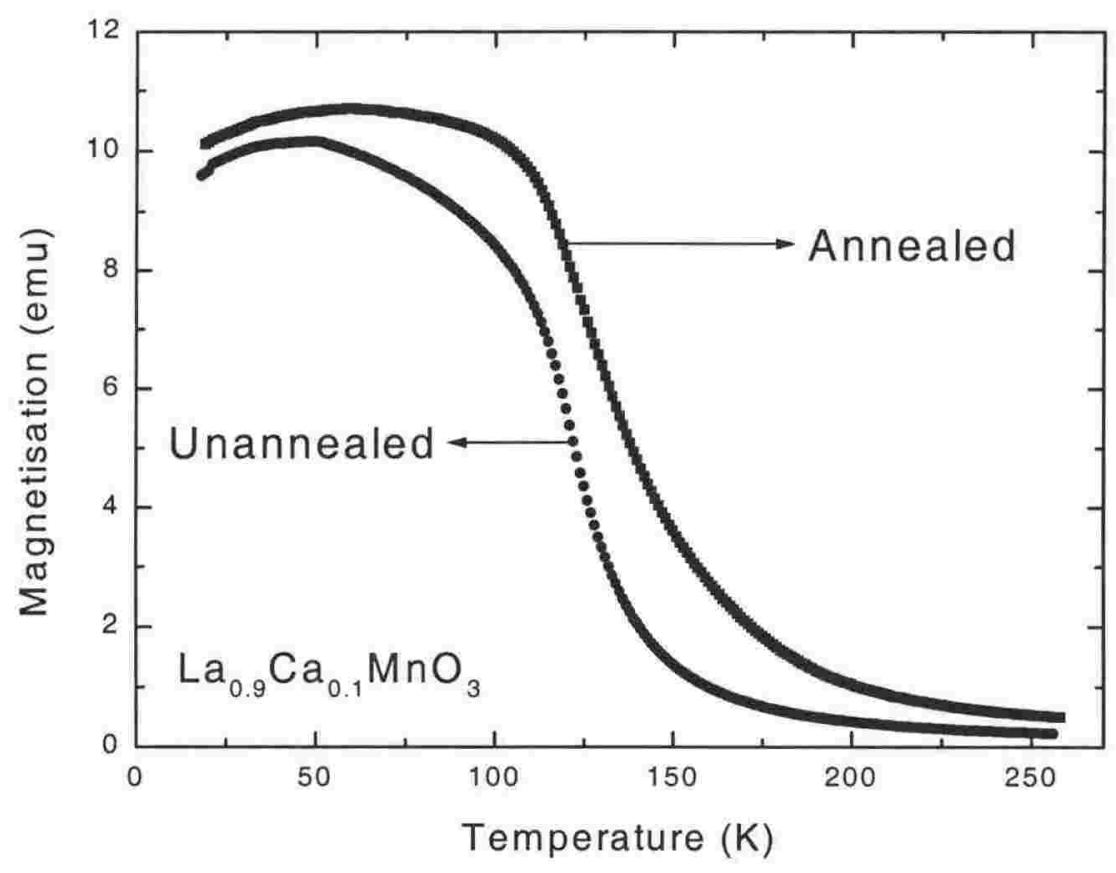

Figure 3.12: Magnetisation with respect to temperature for $\mathrm{La}_{0.9} \mathrm{Ca}_{0.1} \mathrm{MnO}_{3}$ before (circles) and after (squares) annealing.

In measuring the magnetisation change with temperature, the Vibrating Sample Magnetometer at Industrial Research Ltd was used. As well as measuring the temperature-dependent magnetisation of all four samples, in order to find $T_{c}$ and characterise the degree and type of magnetic order in the ma- 
terials, the magnetisation was measured before and after annealing each of the pseudocubic samples to monitor any shifts in $T_{c}$. Description of $M(T)$ will be reserved for discussion alongside optical measurements in Chapter 5. There was a $4 \mathrm{~K}$ increase in $T_{c}$ for the $x=0.265$ substituted sample, as seen in Fig. 3.13, and an increase of approximately $8 \mathrm{~K}$ in $T_{c}$ for the $x=0.1$ substituted sample, as seen in Fig. 3.12. This rather significant increase of $T_{c}$ for $x=0.1$, coupled with a distinct shape-change of the magnetisation, suggests that bulk changes have occurred, possibly structural, consistent also with the extent of cracking in the sample.

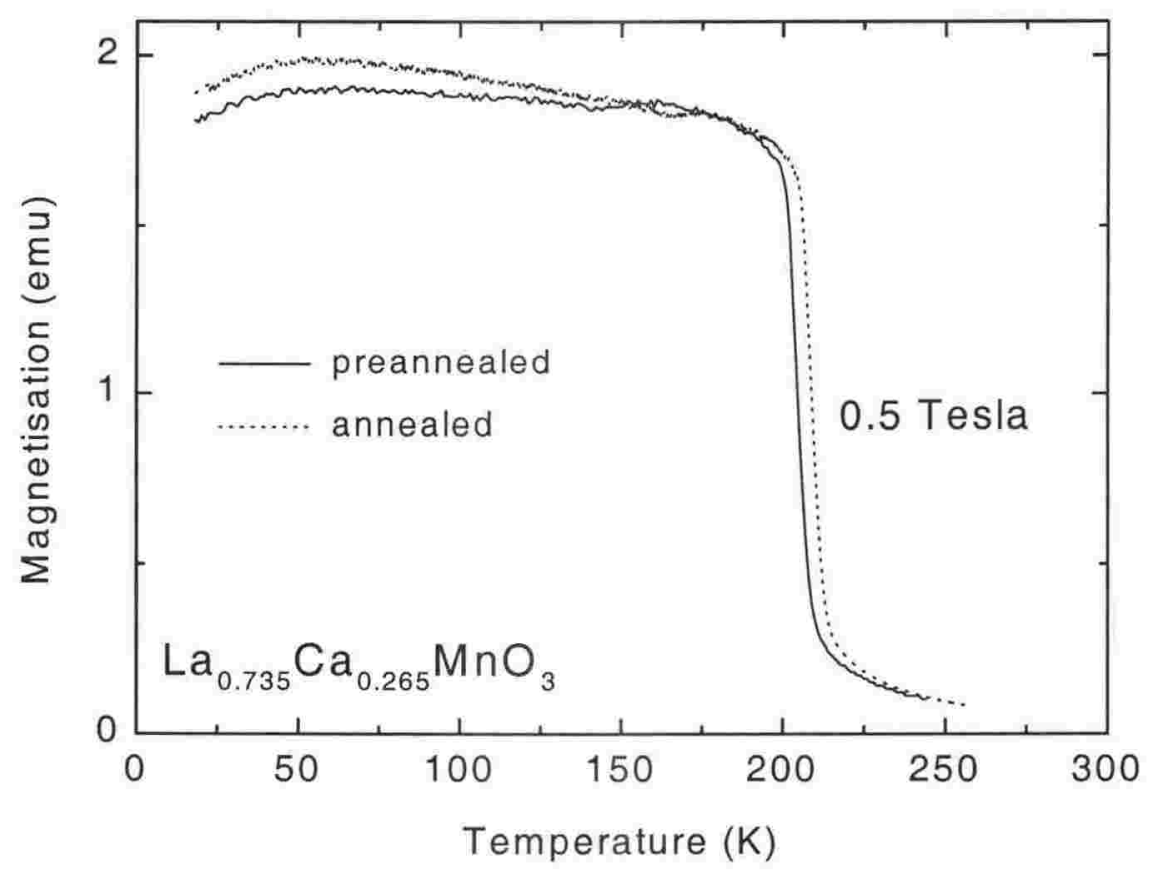

Figure 3.13: Magnetisation with respect to temperature for $\mathrm{La}_{0.735} \mathrm{Ca}_{0.265} \mathrm{MnO}_{3}$ before (solid line) and after (dotted line) annealing. 


\subsubsection{Layered (La/Pr $)_{2-2 x} \mathrm{Sr}_{1+2 x} \mathrm{Mn}_{2} \mathrm{O}_{7}$.}

The layered single crystal materials were also grown by the floating zone method, by a British group at Warwick University, Warwick. The details of this procedure are published elsewhere[59]. Both the $\operatorname{PrSr}_{2} \mathrm{Mn}_{2} \mathrm{O}_{7}$ and the $\mathrm{La}_{1.2} \mathrm{Sr}_{1.8} \mathrm{Mn}_{2} \mathrm{O}_{7}$ samples cleaved along the $a b$ plane, and both presented mirror-like $a b$ plane surfaces of $\approx 3 \mathrm{~mm} \times 4 \mathrm{~mm}$ dimension. In the case of the $\mathrm{PrSr}_{2} \mathrm{Mn}_{2} \mathrm{O}_{7}$ sample this surface was used for both the near-normal incidence reflection and oblique incidence ellipsometry measurements. The $\mathrm{La}_{1.2} \mathrm{Sr}_{1.8} \mathrm{Mn}_{2} \mathrm{O}_{7}$ sample surface was used for ellipsometry only.

The $c a$ planes of the two samples were prepared for optical measurements at the National Synchrotron Light Source by wet polishing in methanol, using grains down to $1.0 \mu \mathrm{m}$. The samples were mounted to a hard plastic brace with GE varnish for the polishing procedure. The polished surface area was about $2 \mathrm{~mm} \times 3 \mathrm{~mm}$ for the $\mathrm{La}_{1.2} \mathrm{Sr}_{1.8} \mathrm{Mn}_{2} \mathrm{O}_{7}$ sample and $1 \mathrm{~mm} \times 4 \mathrm{~mm}$ for the $\mathrm{PrSr}_{2} \mathrm{Mn}_{2} \mathrm{O}_{7}$ sample. The high brilliance of the National Synchrotron Light Source U4IR light source ensured that plenty of reflective signal was achieved for these surfaces, despite the natural wastage of light endemic to the high angles of incidence. 


\section{Chapter 4}

\section{Reflectivity and determining optical constants.}

Our intention is to extract $\sigma(\omega)$ across all frequencies, $\omega$, for which we have measured $R(\omega)$. The central issue in the acquisition of reliable optical constants from normal incidence reflectivity measurements, is how accurately the reflectance can be measured, or modelled, in the 0 to $\infty$ energy range. The piecing together of available reflectivity data and choice of extrapolations to the regions beyond is not trivial. Details of the reflectivity extrapolations used and the subsequent uncertainty in the conductivity from the Kramers-Kronig transform will be dealt with here. Another issue is that, depending on wavelength and refractive index, a significant portion of the material that is probed by reflection spectroscopy is susceptible to surface degradation effects. In order to recover the intrinsic optical properties of a material which probes a proportionately significant surface component compared to the bulk, the surface dielectric constant must be no different to that of the bulk. Thus, as alluded to in section 3.5.2 of the previous chapter, a very careful sample surface preparation regime is of the utmost importance. This chapter will present optical conductivity measurements from surfaces of the pseudocubic manganite which have received both polishing and annealing treatments and discuss the differences. The chapter will conclude with a discussion of the attempted extraction of the $c$-axis dielectric constant from $a b$ plane ellipsometry and normal incidence measurements.

This chapter, then, is something of a halfling: results based, but heavy 
on discussion of the normal incidence reflectivity method in practice. Subsequent chapters will discuss the meatier physics involved in interpreting the optical constants of these manganites.

\subsection{Pseudocubic $\mathrm{La}_{0.9} \mathrm{Ca}_{0.1} \mathrm{MnO}_{3}$.}

$\mathrm{La}_{0.9} \mathrm{Ca}_{0.1} \mathrm{MnO}_{3}$ displays insulator-like resistivities at all temperatures, as inferred by the phase diagram of Fig. 1.4. As such, it displays less colourful optical behaviour when compared to the metallic $\mathrm{La}_{0.735} \mathrm{Ca}_{0.265} \mathrm{MnO}_{3}$ single crystal. For this reason it serves as a good starting point for the step-by-step discussion of the optical constant determination in action.

\subsubsection{Reflectivity spectra}

This section will describe how a reflectivity spectrum of infinite frequency extent was generated from a combination of data and numerical and analytical extrapolations. The reflectivities of the annealed and unannealed samples will then be compared, discussing the similarities and differences.

\section{Extrapolations}

At this point the extrapolations used in the reflectance spectra will be described without rigorous justification. The uncertainty due to the choice of extrapolation will be discussed later in section 4.2.1. Fig. 4.1 displays the reflectivity of the annealed $\mathrm{La}_{0.9} \mathrm{Ca}_{0.1} \mathrm{MnO}_{3}$ single crystal at $295 \mathrm{~K}$. The measured data extends from $65 \mathrm{~cm}^{-1}$ to $45000 \mathrm{~cm}^{-1}$, and consists of data acquired using the FT-IR spectrometer for energies lower than $13000 \mathrm{~cm}^{-1}$, and the grating spectrometer for energies greater than $9000 \mathrm{~cm}^{-1}$, as seen by the overlapping spectra in the inset of Fig. 4.1. The slight mismatch in the level of reflectivity, for the spectra measured with the different spectrometers, has been reconciled by multiplying the infra-red part of the spectrum by 1.1 to give the smooth spectrum in the main panel.

The low energy extrapolation of $R(\omega)$ has been taken as constant, which follows from an assumption that $\sigma_{1}(\omega=0)=0$. The $\mathrm{La}_{0.9} \mathrm{Ca}_{0.1} \mathrm{MnO}_{3}$ sample, 


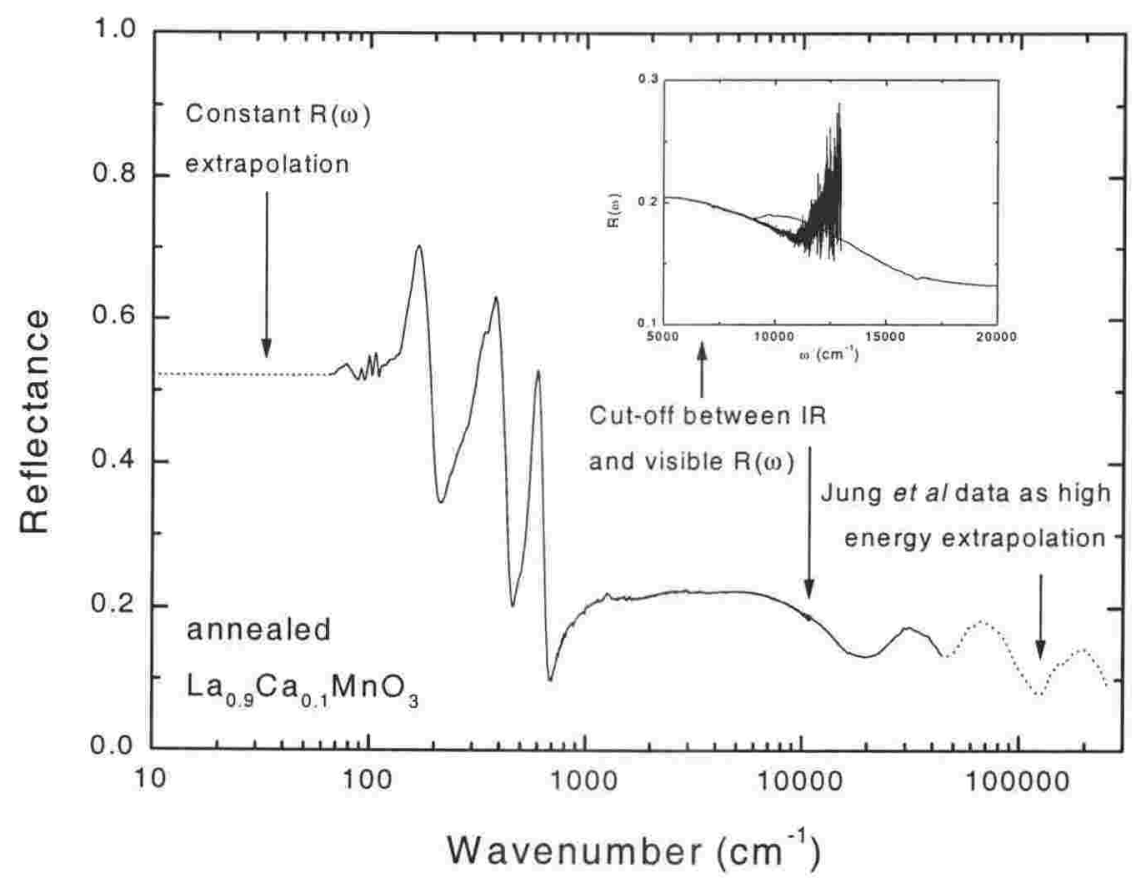

Figure 4.1: Reflectance spectrum (solid line) measured at $295 \mathrm{~K}$ for annealed $\mathrm{La}_{0.9} \mathrm{Ca}_{0.1} \mathrm{MnO}_{3}$. Dashed lines denote the low and high energy extrapolations. The inset shows the contrast between raw IR and visible data in the crossover region.

as an insulator, has a very low $\sigma_{1}(\omega=0)$, and as such is expected to closely approximate a low energy constant reflectivity behaviour, as detailed in section 2.4.1. In any case, as will be demonstrated for the $\mathrm{La}_{0.735} \mathrm{Ca}_{0.265} \mathrm{MnO}_{3}$ sample, the low energy extrapolations have little impact on $\sigma_{\kappa \kappa}(\omega)$ in the region of measured data.

The reflectivity for an unannealed polycrystalline sample of $\mathrm{La}_{0.9} \mathrm{Ca}_{0.1} \mathrm{MnO}_{3}$ measured by Jung and coworkers[23] has been used to bridge the energy gap between $45000 \mathrm{~cm}^{-1}$ and $254000 \mathrm{~cm}^{-1}$. Between 254000 $\mathrm{cm}^{-1}$ and $300000 \mathrm{~cm}^{-1}$ the reflectance has been chosen to decay as $\omega^{-2}$, acknowledging the presence of any higher energy transitions for which no data is available. Beyond $300000 \mathrm{~cm}^{-1}$, or $37 \mathrm{eV}$, the reflectivity is made to decay as $\omega^{-4}$. At this point we simply assert that these extrapolations are 
the most appropriate, deferring a discussion of the influence on the KramersKronig transform of different extrapolations to a later section.

\section{Annealing effects}

The frequency dependent reflectivity, $R(\omega)$, measured for the $x=0.1$ substituted sample at $295 \mathrm{~K}$ before and after annealing, is denoted by dotted and solid lines respectively in Fig. 4.2. Only unannealed reflectivity data were available in the visible region for this sample, so this was spliced to both unannealed and annealed IR data at about $13000 \mathrm{~cm}^{-1}$, as detailed in the previous subsection. Focusing now on the IR region, it can be seen that the annealed and unannealed sample spectra show features in common. Below $800 \mathrm{~cm}^{-1}$ each has 3 large peaks, corresponding to optic phonon modes. A broad peak between $700 \mathrm{~cm}^{-1}$ and $15000 \mathrm{~cm}^{-1}$ appears in the same energy range and at a similar reflectivity level for both annealed and unannealed samples. These general similarities in the spectra aside however, annealing the material does bring about notable changes in the reflectivity. For the unannealed sample, the second phonon mode at $\omega \approx 350 \mathrm{~cm}^{-1}$, in particular, is populated with a number of small peaks. These small peaks are not present in the annealed sample, aside from a small split seen at $\omega \approx 350$ $\mathrm{cm}^{-1}$. The frequency of the mode at $\omega=170 \mathrm{~cm}^{-1}$ has decreased, or "softened", upon annealing. The heights of all three major modes have also decreased with the annealing treatment. The reflectance level increases slightly between $700 \mathrm{~cm}^{-1}$ and $8000 \mathrm{~cm}^{-1}$, then appears to decrease in the visible region, as evidenced by the downturn of the IR reflectivity just above 8000 $\mathrm{cm}^{-1}$. Speculation as to the nature of the changes that have brought about the contrasts in optical features will be left to the next section, in which the conductivity is the focus of discussion.

\subsubsection{Conductivity spectra}

Having extended the $R(\omega)$ spectra to 0 and $\infty$ frequency we are now able to apply the Kramers-Kronig transform and extract optical constants for the annealed and unannealed samples. 


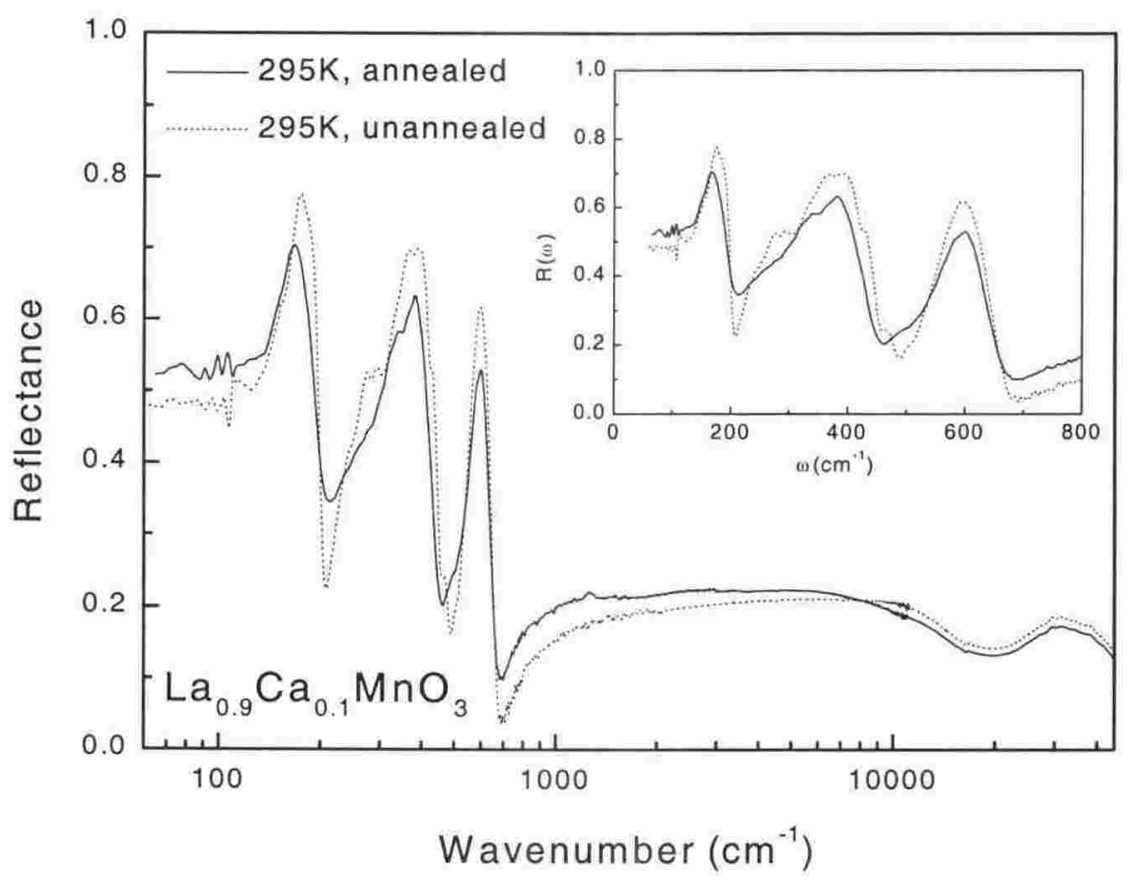

Figure 4.2: Reflectance at $295 \mathrm{~K}$ of $\mathrm{La}_{0.9} \mathrm{Ca}_{0.1} \mathrm{MnO}_{3}$ before and after annealing. The inset shows the phonon region in more detail.

The real part of the Kramers-Kronig transform of $R(\omega)$ will from hereon be labelled, very specifically, as $\sigma_{1, \kappa \kappa}(\omega)$, for reasons which will become clear in the next section. $\sigma_{1, \kappa \kappa}(\omega)$ for the unannealed and annealed $x=0.1$ sample are illustrated with dashed and solid lines respectively in Fig. 4.3. The inset of Fig. 4.3 shows the far-IR $\sigma_{1, \kappa \kappa}(\omega)$ spectra on a linear scale of $\omega$. The three peaks at $\omega \approx 160 \mathrm{~cm}^{-1}, 345 \mathrm{~cm}^{-1}$ and $580 \mathrm{~cm}^{-1}$ have been assigned to the "external", "bending" and "stretching" modes respectively[22]. For the purposes of our description of these modes, we label these the low-energy, mid-energy and high-energy phonons respectively. These will be discussed in more detail in the next chapter. The high-energy phonon has hardened upon annealing, which may betray something of the nature of the oxygen coordination in the material, before and after annealing. The shape of the modes in the phonon region for the annealed $x=0.1$ closely resemble those 


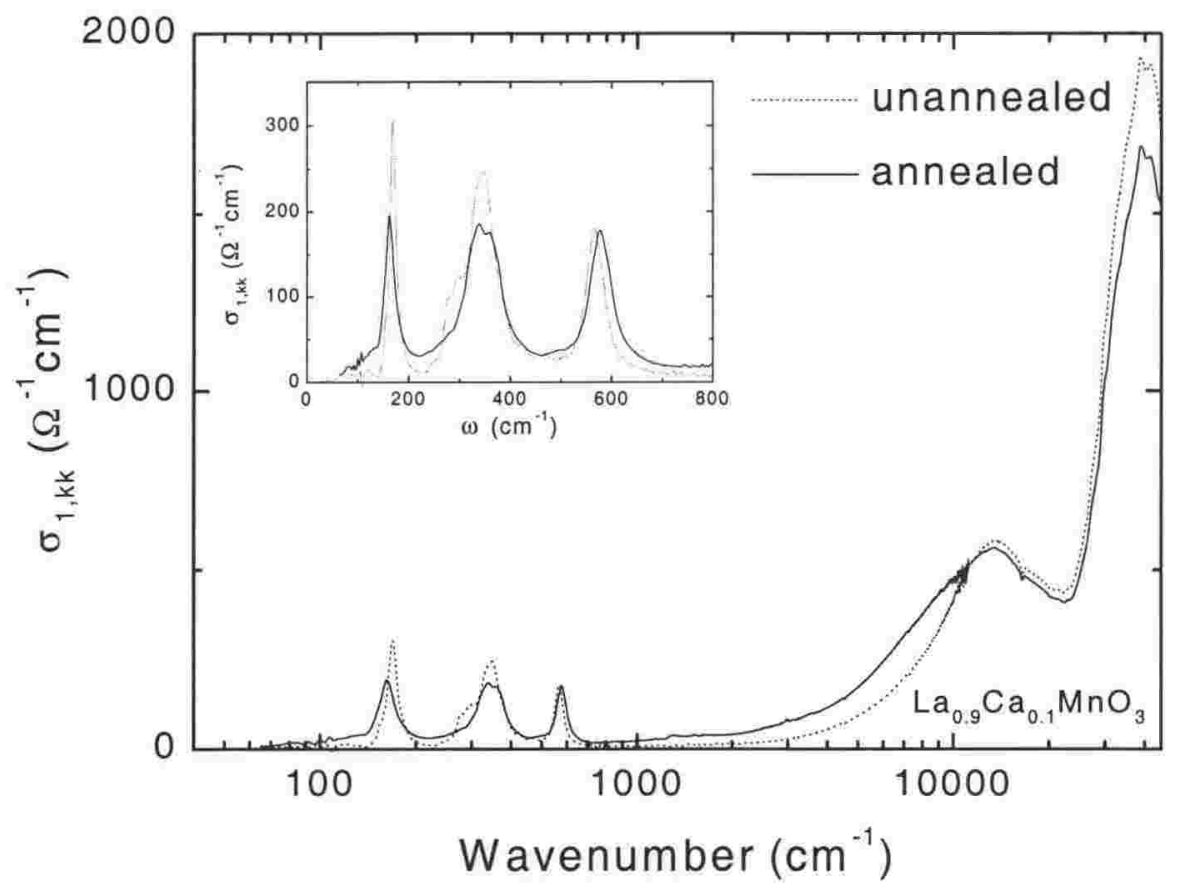

Figure 4.3: $\sigma_{1, \kappa \kappa}(\omega)$ for $\mathrm{La}_{0.9} \mathrm{Ca}_{0.1} \mathrm{MnO}_{3}$ at $295 \mathrm{~K}$ before and after annealing. The inset shows $\sigma_{1, \kappa \kappa}(\omega)$ in the low energy phonon region.

of the measured spectrum for polycrystalline $x=0.1$ [23]. The irregular spiky phonons of the unannealed $x=0.1 \sigma_{1, \kappa \kappa}(\omega)$ spectrum bear more resemblence to the published $x=0$ parent compound $\left(\mathrm{LaMnO}_{3}\right)$ spectrum[23, 24]. The $x=0.1$ spectra are in agreement with published spectra, then, but only after annealing. It appears that the thermal treatment chosen has removed imperfections that were evident in the optical spectra as defect modes.

\subsection{Pseudocubic $\mathrm{La}_{0.735} \mathrm{Ca}_{0.265} \mathrm{MnO}_{3}$.}

The higher level of hole-substitution for $\mathrm{La}_{0.735} \mathrm{Ca}_{0.265} \mathrm{MnO}_{3}$ yields significant temperature dependence in this crystal. As can be seen in the $\mathrm{La}_{1-x} \mathrm{Ca}_{x} \mathrm{MnO}_{3}$ phase diagram of Fig. 1.4 this material undergoes a concurrent insulatormetal, paramagnetic-ferromagnetic transition at $T_{c}$. The resistivity of this material drops through 2 or 3 orders of magnitude, as seen in Fig. 5.1, by 
$10 \mathrm{~K}$ becoming only $2 \times 10^{2}$ times more resistive than copper $(\mathrm{Cu})$ at room temperature. As introduced in section 2.4.2, metallic behaviour is signalled by a broad low energy absorption or Drude-like peak in the optical conductivity spectrum, so we expect to see signs of this in our spectra when the sample temperature is in the metallic phase.

This section will first discuss the extrapolations that were applied to the $\mathrm{La}_{1-x} \mathrm{Ca}_{x} \mathrm{MnO}_{3}(x=0.1,0.265)$ reflectivities more rigorously and the uncertainty that results from our choices. $R(\omega)$ measured from the unannealed sample will be presented to highlight problems of surface preparation, as will surface characterisation measurements of the sample before and after annealing.

\subsubsection{Extrapolations}

Obtaining $\sigma_{\kappa \kappa}(\omega)$ such that it truly represents the intrinsic conductivity of a material relies heavily on the accuracy of the measured $R(\omega)$, and on our choice of $R(\omega)$ outside the measured region. This fact cannot be overstated. The question of which extrapolation is used for the reflectivity is one that must be rigorously justified. As will be shown in this section, even quite similar choices of extrapolation of $R$ can yield very different results for the Kramers-Kronig transformed conductivity. Low energy and high energy extrapolations will be discussed in separate sections.

\section{Low energy extrapolations}

The particular case of the low energy Hagen-Rubens type extrapolation, when applied to reflectivity spectra with metallic-like behaviour, will be discussed in this subsection.

Fig. 4.4(a) shows the low energy reflectance of $\mathrm{La}_{0.735} \mathrm{Ca}_{0.265} \mathrm{MnO}_{3}$ at $75 \mathrm{~K}$ and $190 \mathrm{~K}$. At these temperatures, the sample is in its metallic-like phase $\left(T_{c} \approx 205 \mathrm{~K}\right)$ and the Hagen-Rubens extrapolation is deemed appropriate to use for extending the data to zero energy. Recalling, from section 2.3.3, that this extrapolation only applies when $\epsilon_{2} \gg \epsilon_{1}$, it is prudent to investigate the effect on the Kramers-Kronig transform of variations of the 


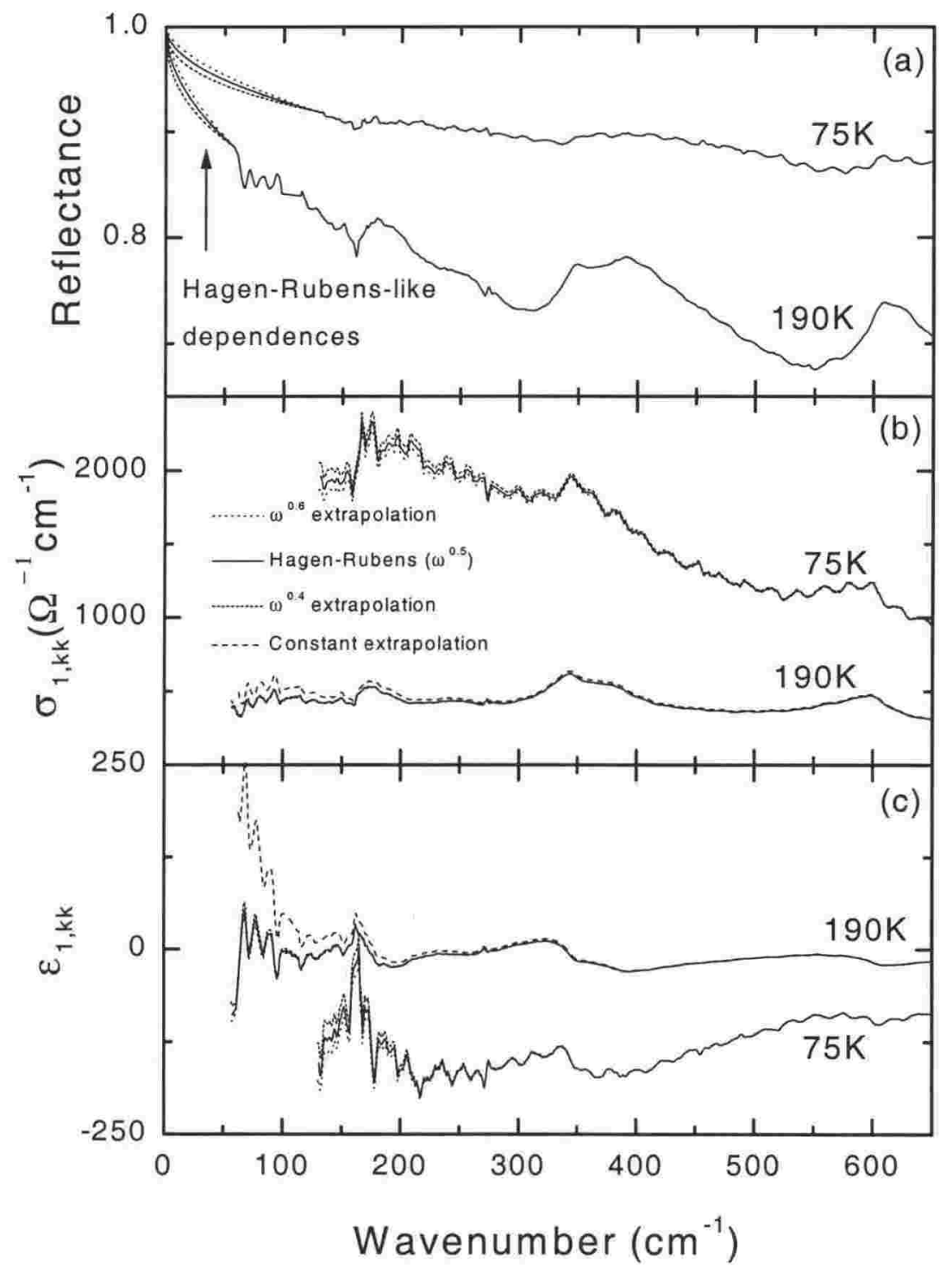

Figure 4.4: (a) Reflectance, (b) $\sigma_{1, \kappa \kappa}(\omega)$, and (c) $\epsilon_{1, \kappa \kappa}(\omega)$ spectra of $\mathrm{La}_{0.735} \mathrm{Ca}_{0.265} \mathrm{MnO}_{3}$ at $75 \mathrm{~K}$ and $190 \mathrm{~K}$ demonstrating the effect of different low energy $R(\omega)$ extrapolations. $\sigma_{1, \kappa \kappa}(\omega)$ and $\epsilon_{1, \kappa \kappa}(\omega)$ for a constant extrapolation of the $190 \mathrm{~K} R(\omega)$ spectrum are pictured as dashed lines, but the $R(\omega)$ extrapolation itself is not shown. Otherwise the legend in the middle graph applies to all panels. 
Hagen-Rubens $\omega^{0.5}$ dependence. For $190 \mathrm{~K}$, in particular, which is close to the metal-insulator transition temperature, the condition that $\epsilon_{2} \gg \epsilon_{1}$ may not be met. The $\omega^{0.5}$ Hagen-Rubens dependence is displayed in Fig. 4.4(a) by solid black lines tagged to the lowest data points of the spectra; $57 \mathrm{~cm}^{-1}$ for $190 \mathrm{~K}$ and $130 \mathrm{~cm}^{-1}$ for $75 \mathrm{~K}$.

To demonstrate the effect on the Kramers-Kronig transform of different reflectivities, different dependences of the low energy reflectance have been used. Illustrated as dotted and short dash lines are, respectively, $\omega^{0.6}$ and $\omega^{0.4}$ extrapolations, which lie either side of the Hagen-Rubens $\omega^{0.5}$ extrapolation curve in Fig. 4.4(a).

It can be seen in panels (b) and (c) that the rather subtle change in reflectivity, from the changes in extrapolation constant, translates to an equally subtle shift in $\sigma_{1, \kappa \kappa}$ and $\epsilon_{1, \kappa \kappa}$, especially for the $190 \mathrm{~K}$ spectrum. As a limiting case for the $190 \mathrm{~K}$ spectrum we attempt an extrapolation involving a constant $R$. Though not shown in Fig $4.4(\mathrm{a}), R(\omega)$ at $63 \mathrm{~cm}^{-1}$ was extended as a straight line to zero frequency, in the manner of the insulating $\mathrm{La}_{0.9} \mathrm{Ca}_{0.1} \mathrm{MnO}_{3}$ samples. $\sigma_{1, \kappa \kappa}$ and $\epsilon_{1, \kappa \kappa}$ that result from this extrapolation are shown with dashed lines in Fig. 4.4 (b) and (c). The effect on $\sigma_{1, \kappa \kappa}$ is minimal, but $\epsilon_{1, \kappa \kappa}$ is changed quite dramatically and, especially for $\omega<150 \mathrm{~cm}^{-1}$, becoming strongly positive, compared to hovering around $\epsilon_{1} \approx 0$ for an Hagen-Rubens extrapolation. In any case, careful scrutiny of Fig. 4.4(b) and (c) reveals that for the $190 \mathrm{~K}$ spectrum, $\epsilon_{2} \approx 500$ (not shown in Fig. 4.4) at the lowest data point, compared with $\epsilon_{1} \approx 0$ (using the Hagen-Rubens extrapolation) and $\epsilon_{1} \approx 200$ (using the constant extrapolation). In the Hagen-Rubens extrapolation case the condition that $\epsilon_{2} \gg \epsilon_{1}$ is met. We thus contend that a Hagen-Rubens extrapolation is appropriate for all spectra at $T<T_{c}$.

\section{High energy extrapolations}

Fig. 4.5(a) shows with solid lines the reflectance of $\mathrm{La}_{0.735} \mathrm{Ca}_{0.265} \mathrm{MnO}_{3}$ at $75 \mathrm{~K}$ and $295 \mathrm{~K}$ - the lowest and highest temperatures measured - for the entire measured energy range. Also shown in Fig. 4.5(a) is the reflectance of non-annealed polycrystalline $\mathrm{La}_{0.7} \mathrm{Ca}_{0.3} \mathrm{MnO}_{3}$, measured by Jung 

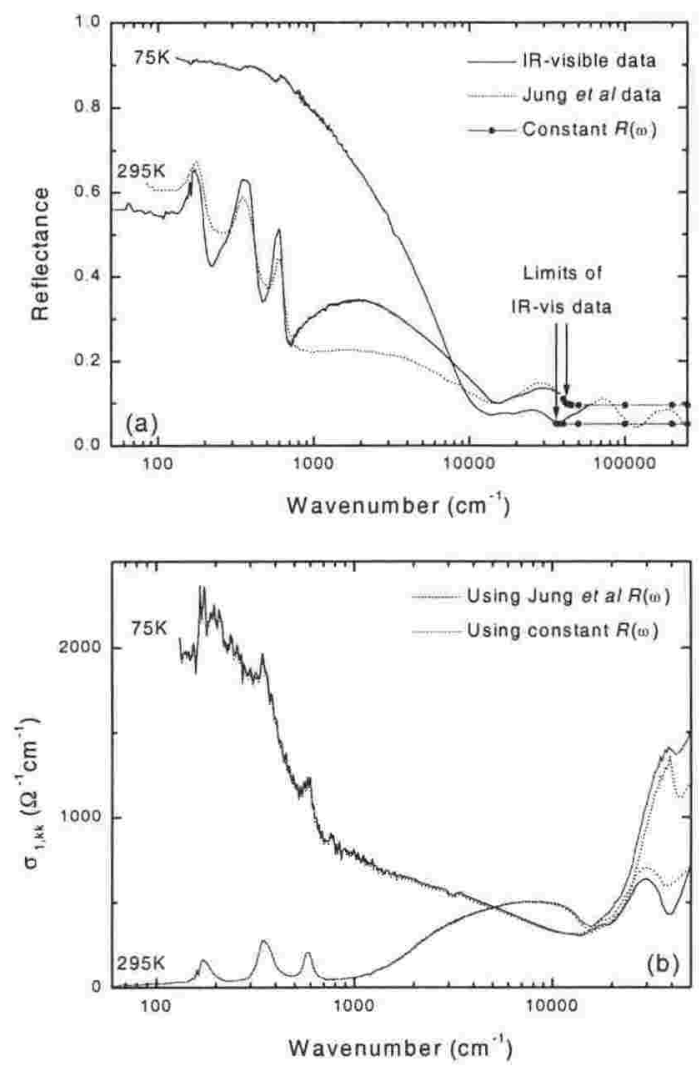

Figure 4.5: (a) Reflectance and (b) $\sigma_{1, \kappa \kappa}(\omega)$ spectra of $\mathrm{La}_{0.735} \mathrm{Ca}_{0.265} \mathrm{MnO}_{3}$ at $75 \mathrm{~K}$ and $295 \mathrm{~K}$ demonstrating the effect on the Kramers-Kronig transform of different high energy $R(\omega)$ extrapolations. Note that the $x$ scales are different and that the legend is split between the two panels.

and coworkers[23] at room temperature. This measurement was made up to $29 \mathrm{eV}$ or $236000 \mathrm{~cm}^{-1}$. The Jung spectrum displayed in Fig. 4.5(a) has been scaled up by 0.02 so that its high energy reflectance is level with our data.

The extrapolation of choice was as detailed for $\mathrm{La}_{0.9} \mathrm{Ca}_{0.1} \mathrm{MnO}_{3}$ : firstly the Jung data is spliced to the end of the temperature-dependent IR-visible data. Between $236000 \mathrm{~cm}^{-1}$ and $300000 \mathrm{~cm}^{-1}$ an $\omega^{-2}$ decay is taken. Beyond $37 \mathrm{eV}$, or $300000 \mathrm{~cm}^{-1}$, an $\omega^{-4}$ decay of $R(\omega)$ is assumed. To investigate the effect on the Kramers-Kronig transform of different high energy extrapolations, a second process went as follows: a constant extrapolation of the reflectance level at the extreme end of the IR-vis data is taken, with an 
$\omega^{-4}$ decay rate beyond $37 \mathrm{eV}$. This can be seen for the two spectra shown illustrated as short dash and circle lines in Fig. 4.5(a).

Because we do not know the exact level of the reflectivity of the annealed single crystal $\mathrm{La}_{0.735} \mathrm{Ca}_{0.265} \mathrm{MnO}_{3}$ in the high energy region, it is anticipated that choosing a constant extrapolation in this region makes a rough approximation to the oscillator strength represented by transitions at these energies. By the same token, the constant extrapolation is also suitably at variance with the Jung data to give us a guide to the uncertainty in the KramersKronig transform.

$\sigma_{1, \kappa \kappa}(\omega)$, for each of the two extrapolation scenarios applied to $75 \mathrm{~K}$ and $295 \mathrm{~K}$, are shown in Fig. 4.5(b). Applying the constant $R$ extrapolation makes significant difference for $\omega>30000 \mathrm{~cm}^{-1}$. Above this energy the $300 \mathrm{~K}$ spectrum shows significant changes in $\sigma_{1, \kappa \kappa}(\omega)$ with different extrapolation choices. For the $75 \mathrm{~K}$ spectrum there are significant variations for $\omega>25000$ $\mathrm{cm}^{-1}$. However, the extrapolation at high energy makes little difference to $\sigma_{1, \kappa \kappa}(\omega)$ in the $65 \mathrm{~cm}^{-1}$ to $25000 \mathrm{~cm}^{-1}$ frequency region, and our quantitative analysis will be confined to within this region. The extrapolations do show us that only qualitative analysis is possible in energy regions above 25000 $\mathrm{cm}^{-1}(3 \mathrm{eV})$. In the energy range below this, the uncertainty in the optical constants due to extrapolation uncertainties, is estimated to be $\pm 1 \%$.

\subsubsection{Annealing effects on $R(\omega)$ and $\sigma_{\kappa \kappa}(\omega)$}

Before it was understood that polishing could skew optical measurements by its damage to a sample surface, $R(\omega)$ spectra were measured from the unannealed sample surface at several temperatures. The spectra at $295 \mathrm{~K}$ and $75 \mathrm{~K}$ are shown in Fig. 4.6(a), and contrasted with the $R(\omega)$ of the annealed sample at the same temperature. Examining first the $295 \mathrm{~K}$ data, the three phonon modes that were apparent in the $x=0.1$ sample are readily observed, though the defect modes of the unannealed $x=0.1$ sample spectra are not visible for the sample in either of its unannealed or annealed states. At $295 \mathrm{~K}$, the level of $R(\omega)$ for the annealed sample is greater than the unannealed sample in the mid-IR region between $800 \mathrm{~cm}^{-1}$ and $6500 \mathrm{~cm}^{-1}$, 


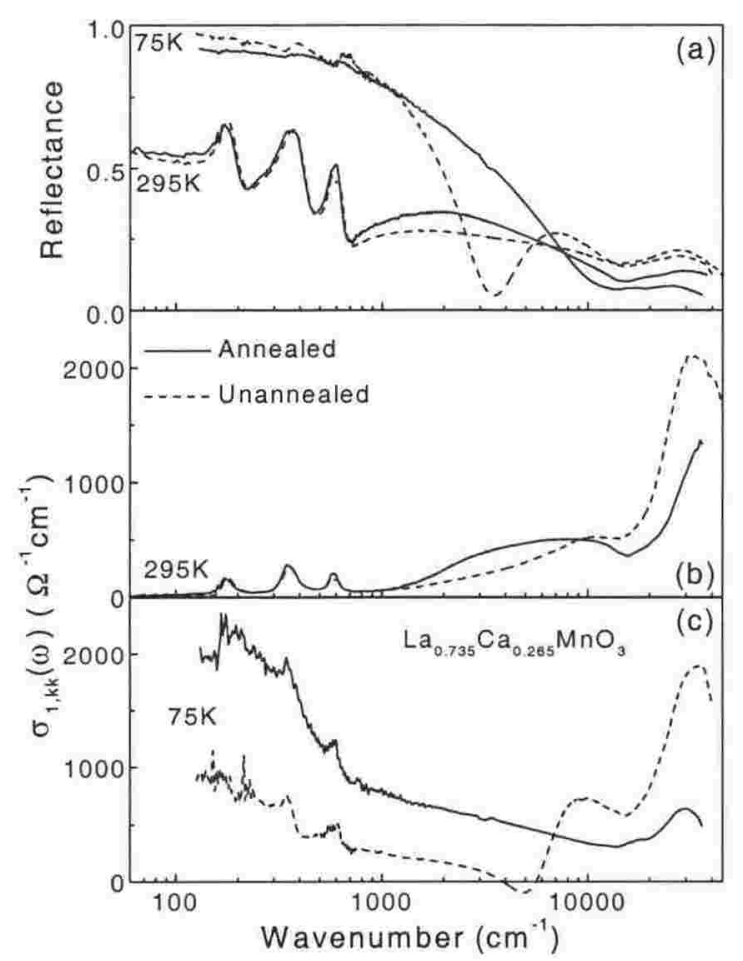

Figure 4.6: (a) $R(\omega)$ and $\sigma_{\kappa \kappa}(\omega)$ spectra at (b) $295 \mathrm{~K}$ and (c) $75 \mathrm{~K}$ for the annealed (solid line) and unannealed (dashed line) $\mathrm{La}_{0.735} \mathrm{Ca}_{0.265} \mathrm{MnO}_{3}$. The legend in the middle graph applies to all panels.

but is decreased in the visible region. This is more likely due to a greater degree of scattering from the surface rather than a decrease in absorption.

These changes are minor, however, in comparison with those seen when the $x=0.265$ sample is in its metallic phase. The energy of the mid-IR minimum in the reflectance, or so-called "plasma edge", more than trebles, as it shifts from $3200 \mathrm{~cm}^{-1}(0.4 \mathrm{eV})$ to $11000 \mathrm{~cm}^{-1}(1.4 \mathrm{eV})$ on annealing. This is a very similar effect to that seen by Lee and coworkers[32], who found that the plasma edge of single crystal $\mathrm{Nd}_{0.7} \mathrm{Sr}_{0.3} \mathrm{MnO}_{3}$ shifted from $0.6 \mathrm{eV}$ to $2 \mathrm{eV}$ upon annealing. Here, this shifted spectral weight absorbs the peak seen in $R(\omega)$ in the unannealed sample at approximately $6500 \mathrm{~cm}^{-1}(0.8 \mathrm{eV})$. The 
$30000 \mathrm{~cm}^{-1}(4 \mathrm{eV})$ peak is still seen in the annealed material but, as for the sample at room temperature, the reflectance level in the visible region has decreased, again predominantly due to scattering rather than a real decrease in oscillator strength. As mentioned in section 3.5.2 the annealing treatment "matted" the surface.

The differences that annealing makes are more concerningly expressed in $\sigma_{1, \kappa \kappa}(\omega)$. The room temperature spectra in Fig. 4.6(b) show an increase in the spectral weight of the mid-IR peak for the annealed sample and a decrease in the spectral weight in the visible region. The changes in the phonon region are relatively small.

However, at $75 \mathrm{~K}$ the spectrum shows huge changes with annealing. As seen in Fig. 4.6(c), the apparent $\sigma_{1, \kappa \kappa}(\omega)$ of the unannealed sample actually goes negative between about $4000 \mathrm{~cm}^{-1}$ and $6000 \mathrm{~cm}^{-1}$. Clearly a negative $\sigma_{1, \kappa \kappa}(\omega)$ is unphysical and needs to be addressed. It is the reason for our dogmatic use of " $\kappa \kappa$ " in the subscript of the $\sigma_{1}$ label. The return of a negative conductivity clearly indicates that $\sigma_{1, \kappa \kappa}$ is at variance with the intrinsic $\sigma_{1} . R(\omega)$ spectra were measured repeatedly, and with different gold reference surfaces, finding variations no greater than our claimed uncertainty. Furthermore, a wide range of both high and low energy extrapolations were tried, finding that an unrealistic high energy extrapolation beyond $40000 \mathrm{~cm}^{-1}(5$ $\mathrm{eV})$ of $\omega^{-18}$ was necessary to drive $\sigma_{1, \kappa \kappa}(\omega)$ positive. It will be shown in the next section that an inhomogeneous dielectric can return negative $\sigma_{1, \kappa \kappa}(\omega)$ values. For the moment we continue our discussion of annealed vs unannealed conductivity spectra. In Fig. 4.6(c), $\sigma_{1, \kappa \kappa}(\omega)$ for the annealed sample not only remains positive, but the extrapolation at the low frequency end of the data, which carries a hugely increased spectral weight, yields $\sigma_{1}(0)=2300 \pm$ $500 \Omega^{-1} \mathrm{~cm}^{-1}$, rather close to the anticipated value from resistivity measurements on the as-grown sample of $\sigma_{d c}=2500 \Omega^{-1} \mathrm{~cm}^{-1}$. The extrapolated zero frequency conductivity of the unannealed sample was about half this value. The resistivity of the annealed material has not been measured, but it was found by Lee and coworkers [32] that the annealing process made very minor changes to $\rho(T)$ in a single crystal of $\mathrm{Nd}_{0.7} \mathrm{Sr}_{0.3} \mathrm{MnO}_{3}$. Further, the effect of annealing on the magnetisation of our sample is negligible. This suggests 
that the bulk properties of the material are not being altered by annealing treatments. A surface effect induced by polishing the sample provides the most consistent explanation for the difference in optical reflectance.

\subsubsection{Modelling of a nonuniform dielectric}

When a repeated reflectivity measurement reproducibly produces negative $\sigma_{1, \kappa \kappa}(\omega)$ from the Kramers-Kronig transform, the next place to lay blame is on the sample itself. It can, in fact, be shown that obtaining a negative $\sigma_{1, \kappa \kappa}$ is not such a preposterous result for a sample that is inhomogeneous, and thus violates one of the assumptions made in the Kramers-Kronig analysis. To demonstrate this, an effective conductivity was calculated for a bulk material with a $0.1 \mu \mathrm{m}$ thick overlay with slightly different optical features from the bulk. The thickness of the overlay was chosen as a conservative estimate of the extent of polishing damages.

Figure 4.7 (a) and (b) show $R(\omega)$ and $\epsilon_{2}(\omega)\left(=4 \pi \sigma_{1}(\omega) / \omega\right)$, respectively, in more detail. The bulk $\epsilon_{2 b}(\omega)$ (dashed line) comprises a Drude peak with $\omega_{p}=1 \mathrm{eV}$ and $\tau=1 \mathrm{eV}^{-1}$, and a Lorentzian oscillator centred at $\omega_{b}=3 \mathrm{eV}$. In addition to those oscillators of the bulk, the layer has a Lorentzian centred at $\omega_{l}=1.5 \mathrm{eV}$ (dotted line). It can be seen that the calculated apparent conductivity $\epsilon_{2 c}(\omega)$ (solid line) from this hybrid material has a distinctly negative component at about $1.2 \mathrm{eV}$. In addition, the Kramers-Kronig transform of the calculated $R(\omega)$ of the hybrid material is shown as solid circles in the inset of Fig. 4.7(b), which magnifies the region where $\epsilon_{2}(\omega)$ goes negative. Though not shown in the main panel $\epsilon_{2, \kappa \kappa}(\omega)=\epsilon_{2 c}(\omega)$ for all $\omega$.

Takenaka and coworkers[61] have suggested that polishing somehow localises the carriers. This is why such a large difference in Drude spectral weight is typically seen between polished and cleaved samples in their metallic phase, and a relatively small difference between polished and cleaved spectra for the insulating phases. This suggestion is certainly borne out in our result. 


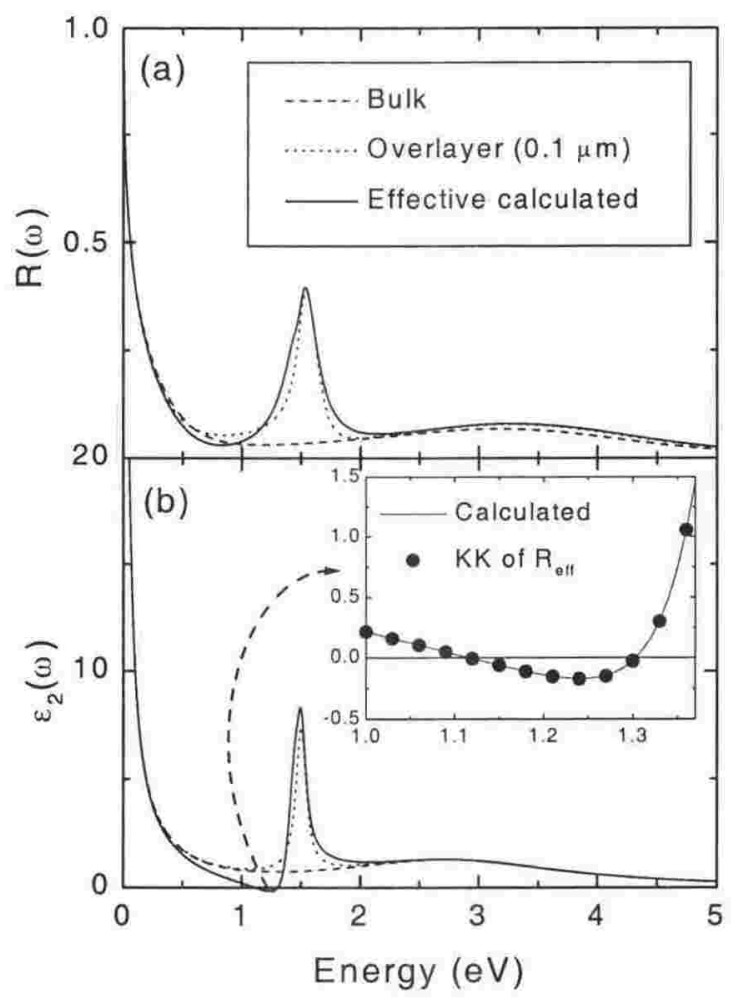

Figure 4.7: (a) $R(\omega)$ and (b) $\epsilon_{2}(\omega)$ for a model material with a $0.1 \mu \mathrm{m}$ overlayer, demonstrating an effective negative conductivity for an inhomogeneous material. The inset to (b) is a close up of the $1.2 \mathrm{eV}$ energy region and includes the Kramers-Kronig transform of $R_{\text {eff }}$.

\subsubsection{Effect of oxygen annealing on surface chemistry}

The differences seen in the optical spectra due to annealing naturally led us to a more thorough examination of the sample surface. A Secondary Ion Mass Spectrometry (SIMS) measurement was made by Jim Metson at Auckland University, NZ (see Fig. 4.8(a),(b)) on the annealed and unannealed $x=0.265$ sample. The composition variation of $\mathrm{La}, \mathrm{Ca}, \mathrm{Mn}$ and $\mathrm{O}$ ions with depth, $z$, below the sample surface is plotted for the $x=0.265$ sample before (a) and after annealing (b). The profiles for each ion have been normalised to their bulk value at $z=220 \pm 20 \mathrm{~nm}$. 


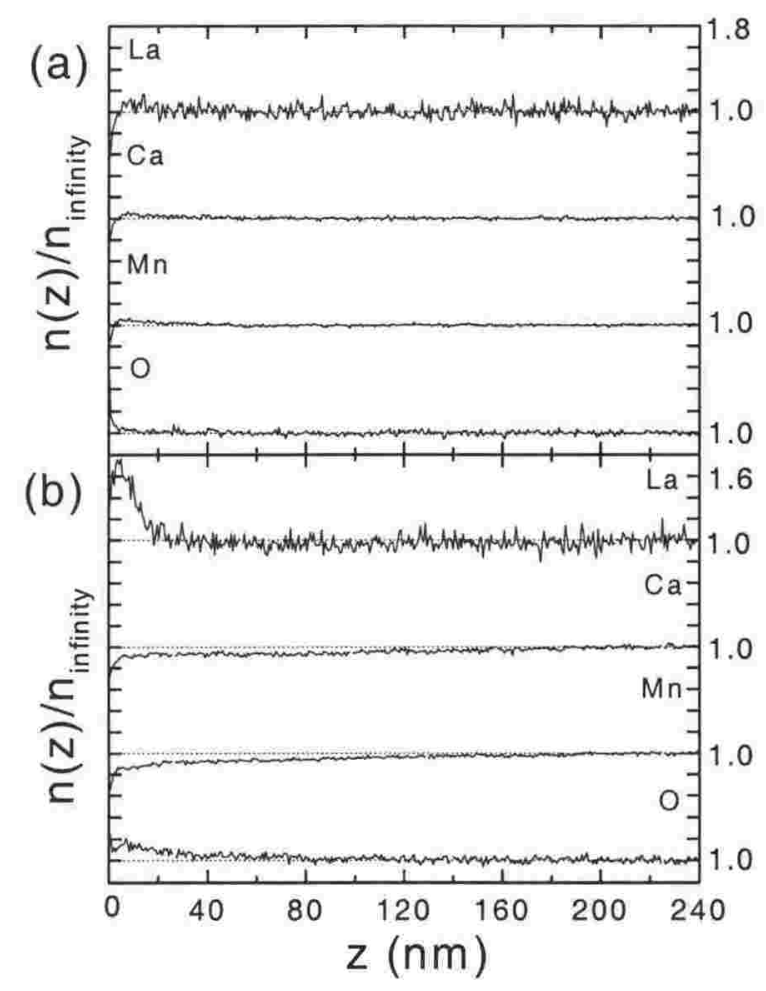

Figure 4.8: Secondary Ion Mass Spectrometry atomic composition profiles for (a) unannealed and (b) annealed $\mathrm{La}_{0.735} \mathrm{Ca}_{0.265} \mathrm{MnO}_{3}$. Horizontal dotted lines at $y=1$ are a guide to the eye. All $y$-axis tick marks denote a spacing of 0.2 .

It can be seen that, in the unannealed sample, the composition of the constituent ions is virtually independent of depth, within uncertainty limits. By contrast, in the annealed sample profiles we see "significant" (denoting damages within $30 \mathrm{~nm}$ of the surface) deviations from the expected bulk compositions for all of the constituent ions near the surface. The La ion in particular is present in quantities of up to $170 \%$ of the unannealed sample near the surface. The average percentage for the ions in depths of $0 \leq z \leq 23$ $\mathrm{nm}$ is $\mathrm{La}=1.35, \mathrm{Ca}=0.91, \mathrm{Mn}=0.88$ and $\mathrm{O}=1.12$. A situation which could favour a surplus of La ions near the surface, accompanied, as it is, by an overpopulation of $\mathrm{O}$ at the surface, is in the possible formation of $\mathrm{LaCO}_{3}$ 


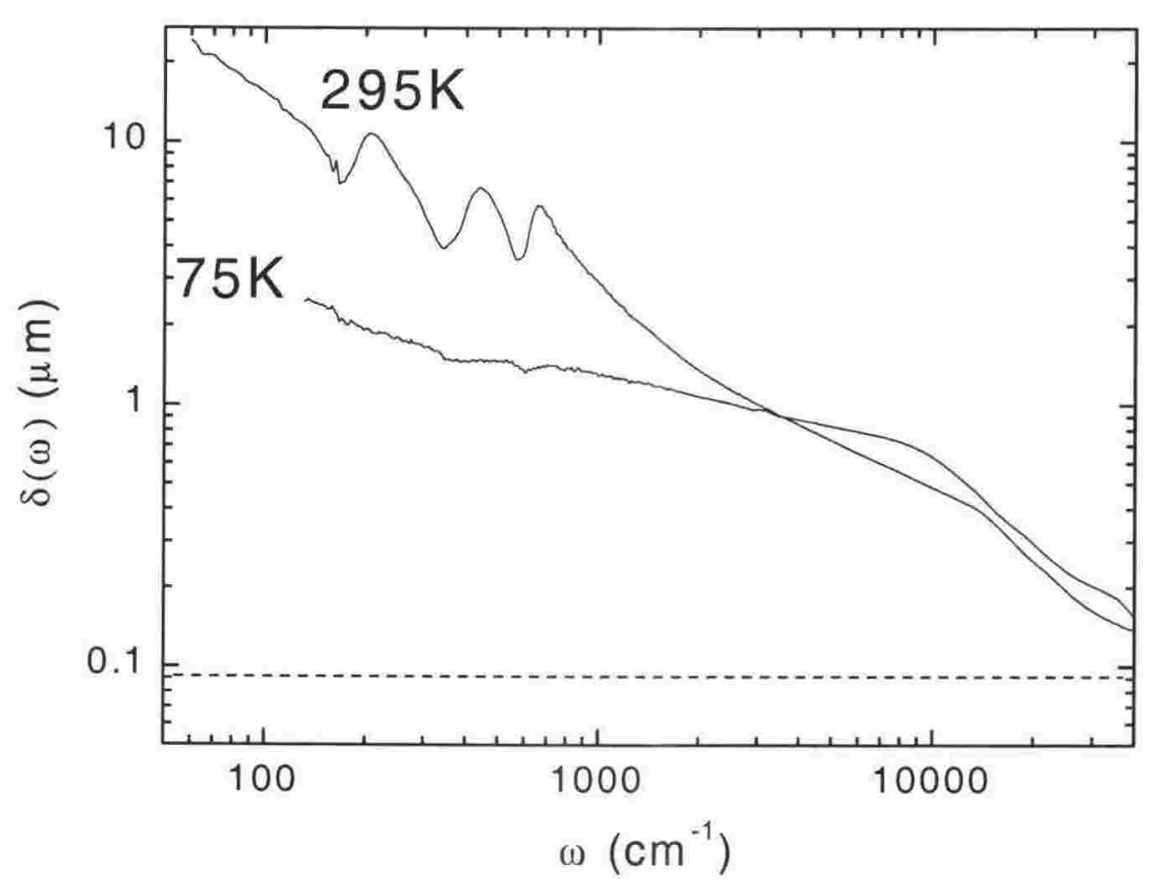

Figure 4.9: Skindepth $\delta(\omega)$ of $\mathrm{La}_{0.735} \mathrm{Ca}_{0.265} \mathrm{MnO}_{3}$ at $295 \mathrm{~K}$ and $75 \mathrm{~K}$. The dashed horizontal line denotes the $z=90 \mathrm{~nm}$ threshhold.

in the annealing process, coexisting with the crystalline perovskite. The Mn and $\mathrm{Ca}$ ion composition deviation is fairly linear, and recovered to 1 ( \pm the uncertainty in the data) at approximately $z=190 \mathrm{~nm}$. For $z \geq 90 \mathrm{~nm}$ the ionic compositions deviate by at most $5 \%$, and we term this a "residual" compositional change.

\subsubsection{Probing light penetration depth}

Although the optical spectra of the unannealed sample are inconsistent with a single interface, Secondary Ion Mass Spectrometry (SIMS) measurements of this surface (Fig. 4.8(a)) show no sign of valence differences that could contribute to the unphysical $\sigma_{1, \kappa \kappa}(\omega)$. This lends even greater credence to the suggestion that strains, not compositional changes, result in a surface layer with a dielectric constant different from the bulk. On the other hand, 
the SIMS measurement of the annealed $x=0.265$ surface, showing significant disturbances $(>10 \%)$ in the valence which persist to depths of $z \approx 30 \mathrm{~nm}$, prevent us from being entirely confident about presenting our annealed sample spectra as representative of the intrinsic optical properties. The question for these spectra then becomes, how significant are the surface changes from the point of view of the light being used to probe the material? To answer this question, the skindepth, $\delta(\omega)$, for this material, was calculated by taking the inverse of the absorption coefficient $\alpha(\omega)=2 \omega k(\omega) / c . k(\omega)$ (the imaginary part of the refractive index) is found from the Kramers-Kronig transform of the annealed $x=0.265$ sample reflectance. As can be seen in Fig. $4.9, \delta(\omega)$, shown at $75 \mathrm{~K}$ and at $295 \mathrm{~K}$, is greater than $90 \mathrm{~nm}$ for all of the frequencies at which data were measured. For frequencies less than $8000 \mathrm{~cm}^{-1}(1 \mathrm{eV})$ $\delta(\omega)$ is at least $450 \mathrm{~nm}$, or five times the depth to which "residual" changes in ionic composition extend, and fifteen times the depth of "significant" ionic composition changes. Of course, we cannot deduce any information about the strains near the surface from SIMS, but the large increase in Drude spectral weight seems to suggest that these have been largely relieved. We venture that for the analysis in the following chapter, which extends to $\omega=0.5 \mathrm{eV}$ (where light probes to a minimum depth of $z=600 \mathrm{~nm}$ ), our data are more than adequately representative of the bulk optical properties.

\subsection{Summary}

Although the idea of modelling an extension of the measured reflectivity, in order to make our data Kramers-Kronig compatible, appears tenuous, and introduces an uncertainty in the conductivity in the region of measured data, this is a quantifiable source of uncertainty, which in many cases is much less than the measurement errors.

We have seen that surface treatments are a major factor in being able to recover the intrinsic conductivity. While polishing damages, evidenced by a dramatically reduced reflectivity in a large energy range, appear repaired by annealing, the SIMS measurement showed us that annealing introduces valence differences near the surface. Quantitative analysis is therefore limited 
to the longer wavelength region. We suggest that surface characterisation measurements should mandatorily accompany optical measurements for annealed samples.

\subsection{Deduction of $c$-axis dielectric constant}

In this section we describe the results of extracting $c$-axis optical constants from normal incidence reflectivity and ellipsometry $a b$ plane measurements. The route taken to arrive at the results was instructive, if the end results in themselves were somewhat disappointing. The mechanics of deducing the $c$-axis optical conductivity, with reflection measurements just from the $a b$ plane, were described in section 2.2.4. In this section we describe the results acquired for the $c$-axis response of the $\mathrm{PrSr}_{2} \mathrm{Mn}_{2} \mathrm{O}_{7}$ sample. This had both normal incidence and oblique incidence reflection measurements made on its $a b$ plane, but no measurements made beyond $700 \mathrm{~cm}^{-1}$ on its $c$-axis. Our aim was to supplement the gap for $\omega>700 \mathrm{~cm}^{-1}$ in the $c$-axis data.

To remind from section 2.2.4, a MATLAB routine was written to calculate the complex frequency-dependent quantity $N_{1}$ from input data and using expression 2.41. $\tilde{n}_{\perp}$ is then found by minimising $a$ in the $f\left(\tilde{n}_{\perp}\right)-N_{1}$ expression of 2.43. In order to test the MATLAB routine a model material, with known uniaxially anisotropic dielectric constants, was used. In this material, the $a b$ plane dielectric constant was made to consist of that of a lorentzian oscillator centred at $350 \mathrm{~cm}^{-1}$. The $c$-axis dielectric constant was contributed to by an identical lorentzian, but centred at $500 \mathrm{~cm}^{-1} \cdot \rho^{\perp}$, or the mixed $a b-c$ plane response due to the anisotropy of $a b$ plane and $c$-axis, was then calculated under the assumption that light was incident on the $a b$ plane at an angle of $75^{\circ}$. The quantities $\rho^{\perp}, \theta$ and $\tilde{n}_{\|}$were then fed into the MATLAB routine, and a $c$-axis response, $n_{\perp}$ and $k_{\perp}$, deduced therefrom. As can be seen in Fig. ?? of Appendix B, the agreement between the fit and the expected $c$-axis response is excellent, indicating that the MATLAB routine and formulae used therein are correct.

Also shown in Appendix B, and in Fig. ??, are the results of another test, involving an $a b$ plane with a Drude dielectric function instead of a Lorentzian. 
Again, the $c$-axis fit and the expected $c$-axis dielectric function were in perfect agreement, and over an energy range from $200 \mathrm{~cm}^{-1}$ to $10000 \mathrm{~cm}^{-1}$. However, for $\omega<200 \mathrm{~cm}^{-1}$ in which $|\operatorname{Im}(N 1)|<\xi$, where $\xi$ is some critical number of the order of 0.01, MATLAB could not converge at a sensible solution. Upon further investigation, it was found that the critical value, $\xi$, appears to be dependent upon the strength of the Drude absorption in the $a b$ plane.

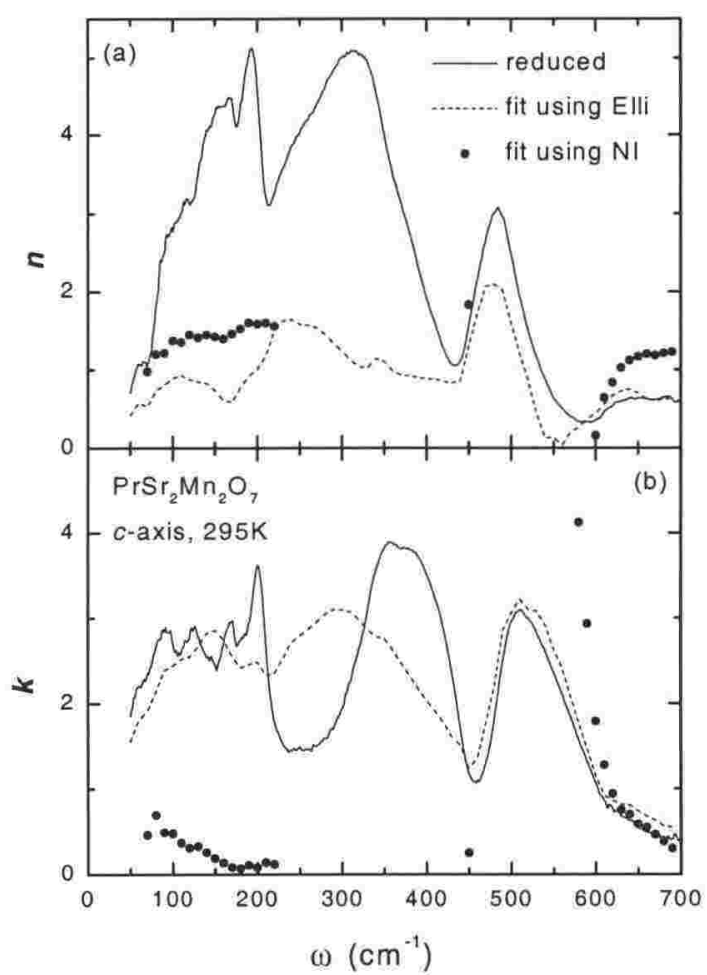

Figure 4.10: $n_{\perp}$ (a) and $k_{\perp}$ (b) found by reduction using $a b-c$ and $c$ - $a b$ ellipsometry measurements (solid line), deduction using reduced $a b$ plane ellipsometry data as $\tilde{n}_{\|}$(dashed line) and deduction using normal incidence reflectivity data as $\tilde{n}_{\|}$(solid circles) for $\mathrm{PrSr}_{2} \mathrm{Mn}_{2} \mathrm{O}_{7}$.

Turning now to an investigation of the measured data, our first approach was to focus on the comprehensively probed far-IR region. In this energy range we are availed of both $a b-c$ and $c-a b$ ellipsometry measurements, which 
enables us to extract the $c$-axis dielectric function by the reduction process. This is illustrated by solid lines for $n_{\perp}$ and $k_{\perp}$ in panel (a) and (b) respectively of Fig. 4.10. Our first fit comes by using the reduced $\tilde{n}_{\|}$from ellipsometry measurements with $\rho^{\perp}$ in expression 2.41. The outcomes of this fit are illustrated as dashed lines in Fig. 4.10, and for most of the spectral range illustrated, show an extremely poor correlation with the expected $\tilde{n}_{\perp}$ (solid lines). Not only is the magnitude of the fit at variance with $\tilde{n}_{\perp}$ for $\omega<450 \mathrm{~cm}^{-1}$, but the shape of the fit is also different.

Still worse, however, is the fit using normal incidence reflectivity measurements as $\tilde{n}_{\|}$, as illustrated by solid circles. The absence of points in many regions of the far-IR is indicative of the difficulty MATLAB had in converging at a solution in these areas. Even in the narrow frequency ranges in which the fit was able to extract sensible numbers, these numbers are, for the most part, far removed from the expected $c$-axis response. The only region in which there is some agreement between fitted and actual $\tilde{n}_{\perp}$ is between $600 \mathrm{~cm}^{-1}$ and $700 \mathrm{~cm}^{-1}$. Given that our aim is to determine the $c$-axis optical constants in the mid-IR, or for $\omega>700 \mathrm{~cm}-1$, it is encouraging for this goal that the agreement of the reduced and fit dielectric constants is tolerable at the high energy end of the far-IR.

We thus now turn to a presentation and discussion of the deduced mid-IR $\tilde{n}_{\perp}$, and display the fit for $\operatorname{PrSr}_{2} \mathrm{Mn}_{2} \mathrm{O}_{7}$ at room temperature and at $75 \mathrm{~K}$ in Fig. 4.11(a). We stress here that this work is shown merely for completion, and not on the basis of any perceived merit in the data itself. In the midIR region, MATLAB converges at a certain solution for $\tilde{n}_{\perp}(\omega)$, which is not Kramers-Kronig-consistent for the region around $1000 \mathrm{~cm}^{-1}$, in which the $75 \mathrm{~K}$ and $295 \mathrm{~K}$ fits go negative. $\sigma_{1}(\omega)$ and $\epsilon_{1}(\omega)$, calculated from the fit $\tilde{n}_{\perp}(\omega)$, are displayed in panels (b) and (c) of Fig. 4.11 respectively. The aforementioned Kramers-Kronig inconsistency around $1000 \mathrm{~cm}^{-1}$ for $k_{\perp}$ is reflected by $\sigma_{1}(\omega)$ going negative. Higher in energy, the shape of $\sigma_{1}(\omega)$ is odd, displaying sharp changes of slope at $1500 \mathrm{~cm}^{-1}$ and $2000 \mathrm{~cm}^{-1}$, for the $295 \mathrm{~K}$ and $75 \mathrm{~K}$ spectra respectively. For all the possible and unquantifiable flaws in the fitted data, it is encouraging that, at the very least, $\epsilon_{1}(\omega)$ is observed to be positive and greater than 1 as the energy increases. 


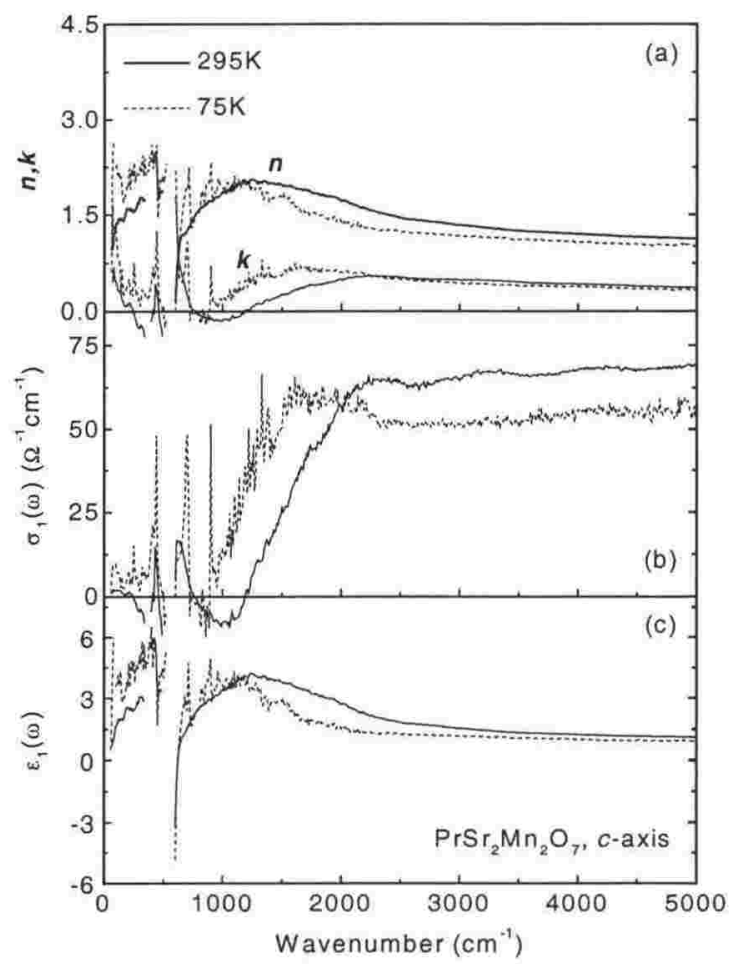

Figure 4.11: mid-IR $c$-axis (a) $\tilde{n}_{\perp}$, (b) $\sigma_{1}(\omega)$ and (c) $\epsilon_{1}(\omega)$ for $\operatorname{PrSr}_{2} \mathrm{Mn}_{2} \mathrm{O}_{7}$, deduced from $a b$ plane measurements.

Our explanation for the large discrepancy between the fits of $\tilde{n}_{\perp}$ using real data involves the uncertainty in the measurement data. Indeed, there is a significant discrepancy between the dielectric constant $\tilde{n}_{\|}$measured by ellipsometry and by normal incidence reflection. This can be seen by direct comparison of $R(\omega)$ and $\sigma_{1}(\omega)$, measured for $\operatorname{PrSr}_{2} \mathrm{Mn}_{2} \mathrm{O}_{7}$ by normal incidence reflectivity and ellipsometry, in Appendix C. Although not displayed as $n$ and $k$ there, $k_{\|}$differs by up to $80 \%$ between normal and oblique incidence measurements. We investigated the effect of enforcing uncertainties onto the model data, and found that significant shifts in the fits for $\tilde{n}_{\perp}$ can result.

Further, the MATLAB routine is written without regard to Kramers- 
Kronig consistency. This means that for a simple model in which the solution is known, and the "measurements" are exact, the best fit arrived at is the expected one. Enforcing errors onto the "measurement" can bring about significant shifts of the solution curve from the correct one, destroying the applicability of the dispersive relationship between measurements.

We might anticipate that the relative success of fitting in the region between $450 \mathrm{~cm}^{-1}$ and $700 \mathrm{~cm}^{-1}$ is due to the $c$-axis component interacting strongly with the $a b$ plane component. Investigations using the model materials have shown that if $\rho^{\perp}$ and $\tilde{n}_{\|}$of the model material are well known, the degree of interaction does not matter. $\tilde{n}_{\perp}$ is returned exactly, or not at all. However, it seems a reasonable suggestion that uncertainties in the measured data affect the fitting result proportionately less in regions of strong $c$-axis interactive component. Although more work is needed, it seems safe to conclude that the greater the interaction of the $c$-axis with the $a b$ plane measurement, the more likely the fit is to succeed in finding a solution.

Thus, the usefulness of this method for extracting $c$-axis mid-IR data from our $a b$ plane measurements, though potentially valuable, is also severely restricted by uncertainties in the datasets. 


\section{Chapter 5}

\section{Optical conductivity.}

...in thy light shall we see light. Psalm 36:9.

Having determined optical constants, through either a Kramers-Kronig transform of the normal incidence reflectivity or from the reflection phase change in the oblique incidence reflectance measurement, we may now discuss these spectra in detail and discover what may be learned from the absorption features displayed. Especially of interest is the temperature-dependence of phonon and electronic contributions to the conductivity, and signatures of interplay between the electrons and the lattice.

The measurements on members of the $n=\infty$ and $n=2$ layered materials will be presented in a specific order: the most weakly and strongly metallic materials, respectively $\mathrm{La}_{0.9} \mathrm{Ca}_{0.1} \mathrm{MnO}_{3}$ and $\mathrm{La}_{0.735} \mathrm{Ca}_{0.265} \mathrm{MnO}_{3}$, will be discussed first (see Fig. 5.1 for resistivity information on these samples). The bi-layered manganites will then be discussed in order of decreasing conductivity; $\mathrm{La}_{1.2} \mathrm{Sr}_{1,8} \mathrm{Mn}_{2} \mathrm{O}_{7}$, having a metal-insulator transition at $T_{c}=125 \mathrm{~K}$ and then $\operatorname{PrSr}_{2} \mathrm{Mn}_{2} \mathrm{O}_{7}$, expected to show insulator-like behaviour.

The measurements that have been made are summarised in Table 5.1, showing for each of the four samples which of the normal incidence reflectivity (NI) and ellipsometry (Elli) experimental techniques were used, the energies at which the experiments were performed and the temperatures at which spectra were acquired.

Fig. 5.1 displays the DC electron transport properties of three of the samples investigated. The $\mathrm{La}_{0.9} \mathrm{Ca}_{0.1} \mathrm{MnO}_{3}$ sample behaves as an insula- 


\begin{tabular}{|c|c|c|c|c|}
\hline Sample & $x$ & Expt. & Energy $\left(\mathrm{cm}^{-1}\right)$ & Temp. \\
\hline \multirow[b]{2}{*}{$\mathrm{La}_{1-x} \mathrm{Ca}_{x} \mathrm{MnO}_{3}$} & 0.1 & NI & $\begin{array}{c}9000-50000 \\
60-15000\end{array}$ & $\begin{array}{c}295 \mathrm{~K} \\
70 \mathrm{~K}-295 \mathrm{~K}\end{array}$ \\
\hline & 0.265 & NI & $\begin{array}{c}9000-50000 \\
9000-37000 \\
60-13000 \\
\end{array}$ & $\begin{array}{c}295 \mathrm{~K} \\
80 \mathrm{~K}, 150 \mathrm{~K} \\
75 \mathrm{~K}-295 \mathrm{~K} \\
\end{array}$ \\
\hline \multirow{2}{*}{$\begin{array}{r}\mathrm{La}_{2-2 x} \mathrm{Sr}_{1+2 x} \mathrm{Mn}_{2} \mathrm{O}_{7} \\
a b \text { plane } \\
c \text {-axis }\end{array}$} & \multirow{2}{*}{0.4} & Elli & $50-5000$ & $10-295 \mathrm{~K}$ \\
\hline & & Elli & $\begin{array}{c}50-5000 \\
50-700\end{array}$ & $\begin{array}{c}295 \mathrm{~K} \\
10-295 \mathrm{~K}\end{array}$ \\
\hline \multirow{2}{*}{$\begin{array}{r}\mathrm{Pr}_{2-2 x} \mathrm{Sr}_{1+2 x} \mathrm{Mn}_{2} \mathrm{O}_{7} \\
a b \text { plane } \\
\text { c axis }\end{array}$} & \multirow[t]{2}{*}{0.5} & $\begin{array}{l}\text { Elli } \\
\text { NI }\end{array}$ & $\begin{array}{c}50-5000 \\
60-13000 \\
\end{array}$ & $\begin{array}{c}10-295 \mathrm{~K} \\
70 \mathrm{~K}, 140 \mathrm{~K}, 295 \mathrm{~K}\end{array}$ \\
\hline & & Elli & $50-700$ & $10-295 \mathrm{~K}$ \\
\hline
\end{tabular}

Table 5.1: Summary, for each sample, of the energy and temperature ranges at which the two experiments were performed.

tor, its resistivity increasing as the temperature decreases. Likewise, the resistivity of the $c$-axis of $\mathrm{La}_{1.2} \mathrm{Sr}_{1.8} \mathrm{Mn}_{2} \mathrm{O}_{7}$, the $a b$ plane of $\mathrm{La}_{1.2} \mathrm{Sr}_{1.8} \mathrm{Mn}_{2} \mathrm{O}_{7}$ and $\mathrm{La}_{0.735} \mathrm{Ca}_{0.265} \mathrm{MnO}_{3}$ display this insulator-like behaviour for temperatures above $125 \mathrm{~K}, 125 \mathrm{~K}$ and $205 \mathrm{~K}$, or the $T_{M I}$, respectively. However, as the temperature is decreased through a narrow temperature band from these $T_{M I}$, the resistivity drops by 2 orders of magnitude in $\mathrm{La}_{1.2} \mathrm{Sr}_{1.8} \mathrm{Mn}_{2} \mathrm{O}_{7}$ and at least an order of magnitude in $\mathrm{La}_{0.735} \mathrm{Ca}_{0.265} \mathrm{MnO}_{3}$. The resistivity of $\mathrm{La}_{0.735} \mathrm{Ca}_{0.265} \mathrm{MnO}_{3}$ continues to decrease in a metallic fashion (as we have chosen to describe "metallic" behaviour in Chapter 1) with decreasing temperature, whilst the $\mathrm{La}_{1.2} \mathrm{Sr}_{1.8} \mathrm{Mn}_{2} \mathrm{O}_{7}$ sample is metallic-like to about $50 \mathrm{~K}$. Below this temperature, there is a slight upturn in $\rho(T)$. For $\mathrm{La}_{0.735} \mathrm{Ca}_{0.265} \mathrm{MnO}_{3}$ at $10 \mathrm{~K}, \rho_{d c} \approx 30 \mu \mathrm{m}$, or just 2 orders of magnitude greater than the room temperature resistivity of copper. 


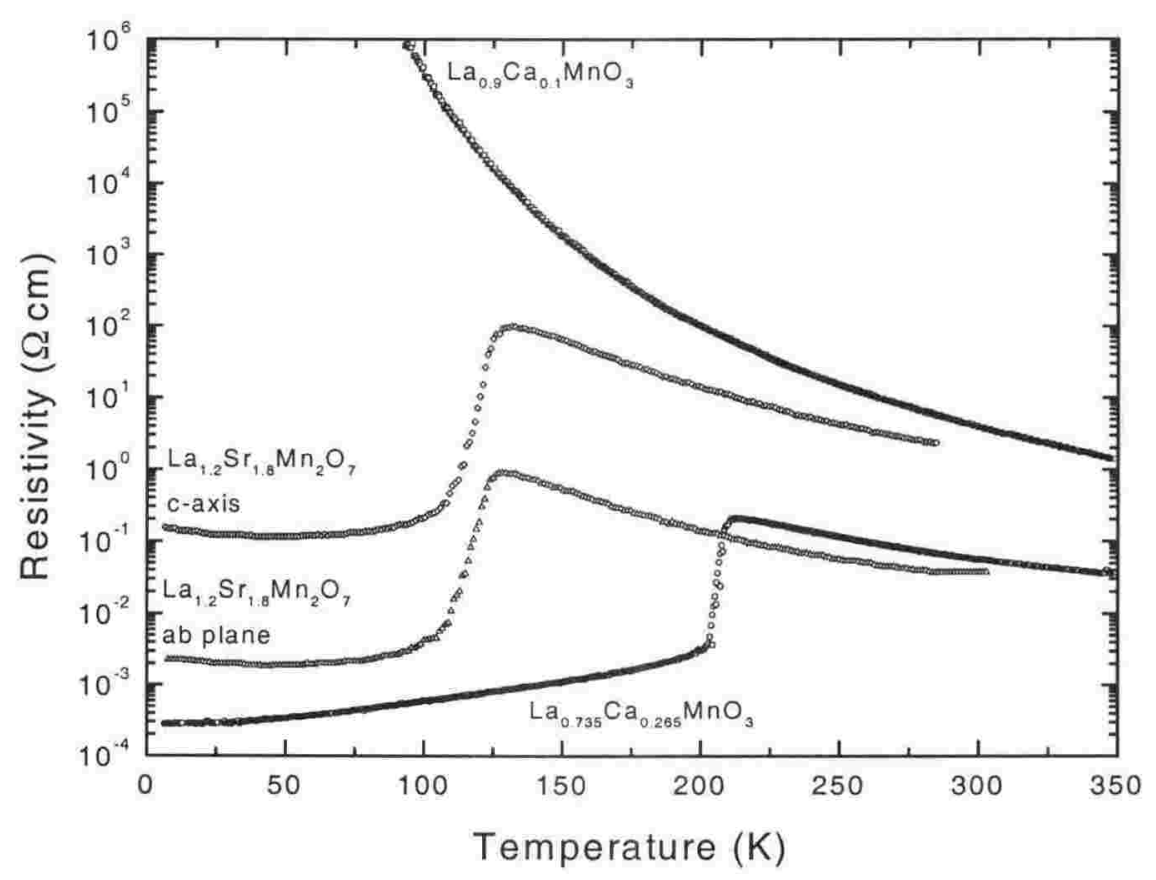

Figure 5.1: Resistivity as a function of temperature $(H=0)$ for $\mathrm{La}_{1-x} \mathrm{Ca}_{x} \mathrm{MnO}_{3}(x=0.1,0.265)$ from [62] and both $a b$ plane and $c$-axis of $\mathrm{La}_{1.2} \mathrm{Sr}_{1.8} \mathrm{Mn}_{2} \mathrm{O}_{7}$ from ref. [63].

\subsection{Pseudocubic $\mathrm{La}_{1-x} \mathrm{Ca}_{x} \mathrm{MnO}_{3}$.}

\subsection{1 $\sigma_{1, \kappa \kappa}(\omega, T)$ for $\mathrm{La}_{0.9} \mathrm{Ca}_{0.1} \mathrm{MnO}_{3}$}

In section 4.1 of the last chapter, the differences between room temperature annealed and unannealed samples were discussed. The appearance of small peaks on the three main phonons were ascribed to defects which are removed by annealing. Here, for completion, we display in Fig. 5.2 a comparison of the already introduced $295 \mathrm{~K} \sigma_{1, \kappa \kappa}(\omega)$ spectrum with that measured at $70 \mathrm{~K}$, for the unannealed sample. The only temperature-dependence observed is in a slight broadening of the modes as the temperature is increased, ascribable to anharmonic effects. It is reasonable to assume that this minimal temperaturedependence would also be observed in the annealed sample. 


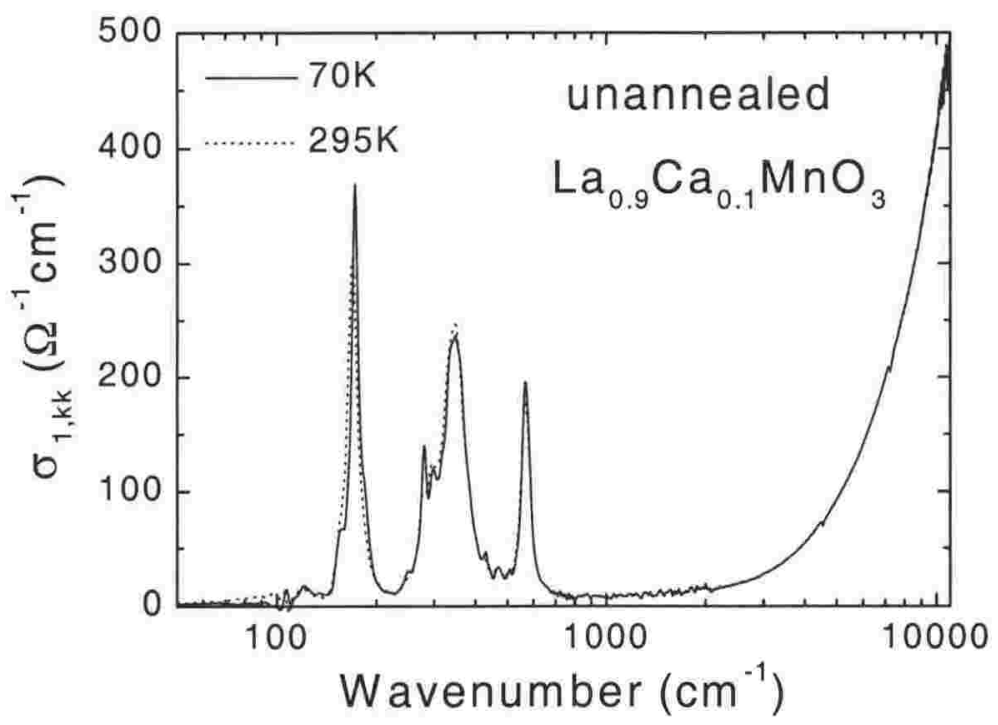

Figure 5.2: $\sigma_{1, \kappa \kappa}(\omega)$ measured for the unannealed $\mathrm{La}_{0.9} \mathrm{Ca}_{0.1} \mathrm{MnO}_{3}$ sample at $70 \mathrm{~K}$ and $295 \mathrm{~K}$.

\subsection{2 $\mathrm{La}_{0.735} \mathrm{Ca}_{0.265} \mathrm{MnO}_{3}$}

$\mathbf{R}(\omega, T)$

Figure 5.3 shows $R(\omega)$ measured for the annealed $\mathrm{La}_{0.735} \mathrm{Ca}_{0.265} \mathrm{MnO}_{3}$ at various temperatures between $75 \mathrm{~K}$ and $295 \mathrm{~K}$ and in the frequency range of 40-45000 $\mathrm{cm}^{-1}$. At $295 \mathrm{~K}$ and $255 \mathrm{~K}$, that is, for $T>T_{c}$, the phonons are very prominent. The mid-IR region shows a broad peak and the high energy region shows a peak in the reflectance at about $30000 \mathrm{~cm}^{-1}(4 \mathrm{eV})$.

As the temperature is decreased, the three phonon peaks in the far-IR become progressively obscured by the increasing low energy reflectivity background. The magnitude of $R(\omega)$ approaches 1 as $\omega$ tends to 0 . The reflectance shows strong temperature dependence, even at the $30000 \mathrm{~cm}^{-1}$ peak, which displays a shift in energy as well as a change in magnitude (discounting the $190 \mathrm{~K}$ visible spectrum, which is scaled to better coincide with the IR data). The other interesting feature is a peak at about $6000 \mathrm{~cm}^{-1}(0.7 \mathrm{eV})$, seen in the $190 \mathrm{~K}$ and $160 \mathrm{~K}$ spectra. This feature becomes obscured by the increasing Drude-like $R(\omega)$ as the temperature decreases. 


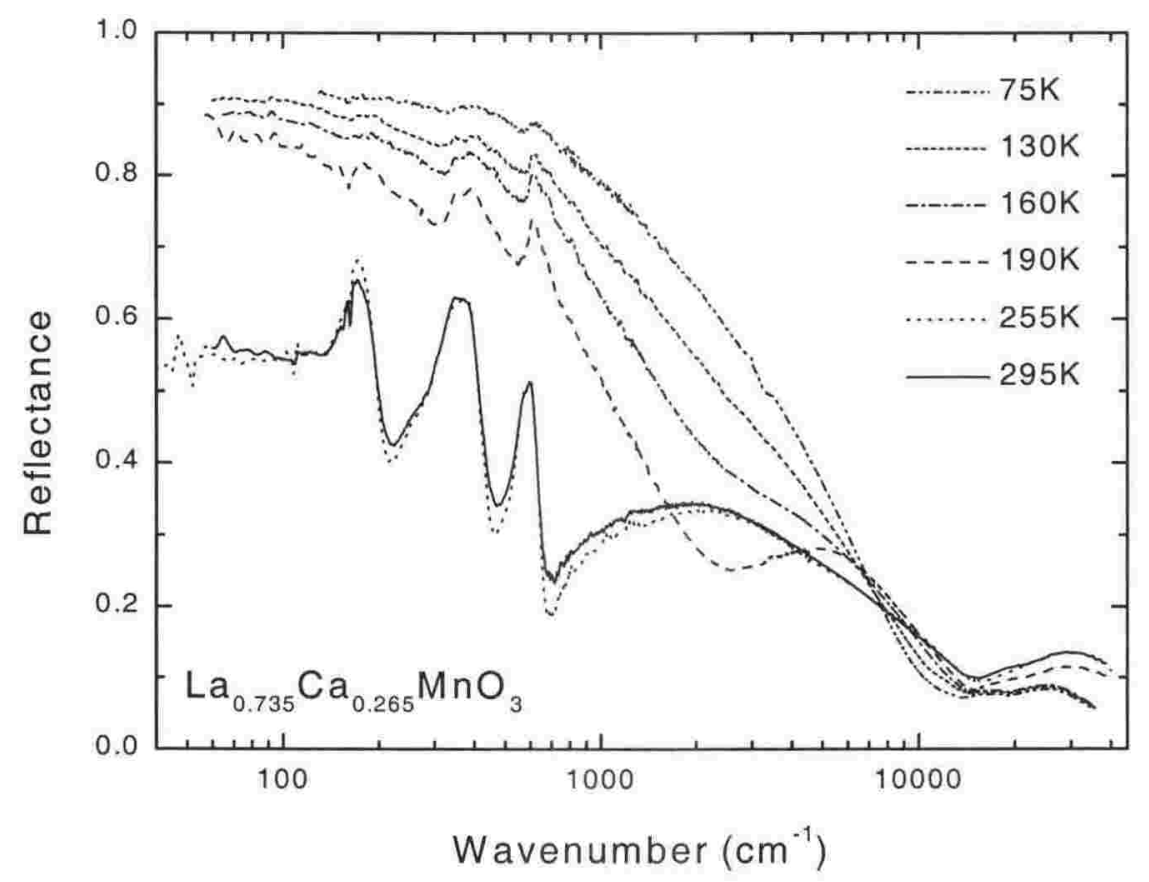

Figure 5.3: $R(\omega)$ measured for the annealed $\mathrm{La}_{0.735} \mathrm{Ca}_{0.265} \mathrm{MnO}_{3}$ sample.

$\sigma_{1, \kappa \kappa}(\omega, T)$

The Kramers-Kronig transforms of the $R(\omega, T)$ spectra are shown in Fig. 5.4 in the energy region $45-45000 \mathrm{~cm}^{-1}$. Plotted as open circles on the $\mathrm{y}$-axis are zero-frequency conductivity values deduced from the DC resistivity measurements displayed in Fig. 5.1. The sample at $295 \mathrm{~K}$ and $255 \mathrm{~K}$ (that is, for $T>T_{c}$ ) shows a gap-like feature at $800 \mathrm{~cm}^{-1}$. This feature is also seen at this energy in the works of Boris[64] and Kim[65], respectively on single crystal and polycrystalline samples of approximately a third substituted $\mathrm{La}_{1-x} \mathrm{Ca}_{x} \mathrm{MnO}_{3}$. As illustrated in Fig. 1.9, the three low energy phonon modes seen in all of the manganite compounds have been ascribed to (in order of increasing energy) "external" ("rare earth"), "bending", and "stretching" modes of the $\mathrm{MnO}_{6}$ octahedra[22]. We adopt the labels lowenergy, mid-energy and high-energy respectively to describe these modes, whose energies are given later, in Table 5.2. The $295 \mathrm{~K}$ and $255 \mathrm{~K}$ spectra 


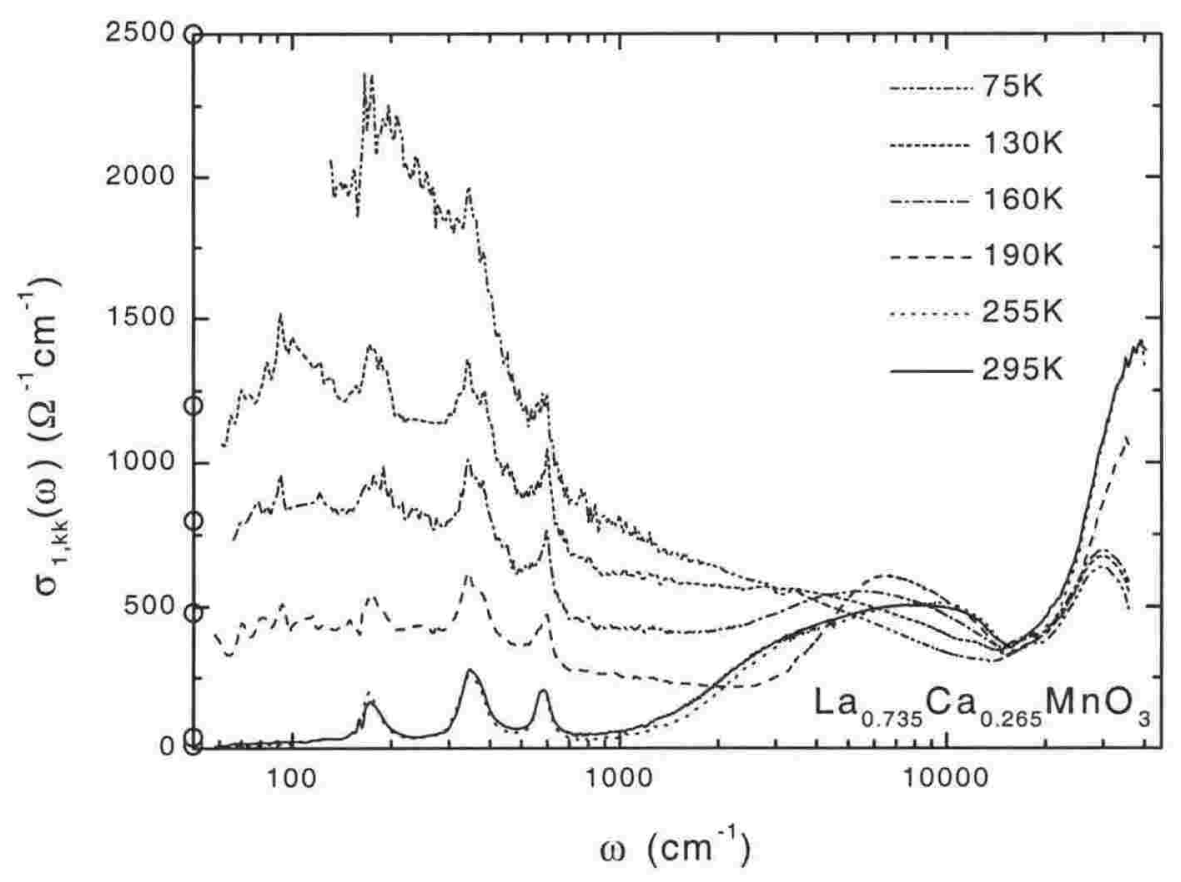

Figure 5.4: $\sigma_{1}(\omega)$ found from a Kramers-Kronig transform of $R(\omega)$ for the annealed $\mathrm{La}_{0.735} \mathrm{Ca}_{0.265} \mathrm{MnO}_{3}$ sample.

are also characterised by two higher energy peaks, a broad one at about 8000 $\mathrm{cm}^{-1}(1 \mathrm{eV})$ and the other at about $30000 \mathrm{~cm}^{-1}\left(4 \mathrm{eV}\right.$.) The $30000 \mathrm{~cm}^{-1}$ $(4 \mathrm{eV})$ peak has been alternately ascribed to the charge-transfer transition between $\mathrm{O}_{2 p}$ and $\mathrm{Mn} e_{g}$ bands[23, 29,32] and a transition between $\mathrm{O}_{2 p}$ and Mn $t_{2 g}$ bands $[24,25,34]$. Our observation of temperature-dependence in this feature favours the interpretation of the feature as $e_{g}$ related. For $T>T_{c}$, the $e_{g}$ electron is localised. For $T<T_{c}$ it becomes itinerant. We find the broad feature at $8000 \mathrm{~cm}^{-1}(1 \mathrm{eV})$ near impossible to fit with just one oscillator, and there is some speculation in the literature as to whether this peak is single[29, 32]. Fitting has not been attempted here; due to their similarities, it is just as instructive to compare the present dataset with literature spectra. Our attempts to fit the low-temperature spectra with Drude and Lorentzian features are also not included here. The uncertainty in de- 
termining the plasma frequency, $\omega_{p}$, and scattering rate, $\tau$, from the Drude component is large, due to the difficulty in obtaining a good fit of the data to a Drude absorption. In any case, the electron dynamics of the manganites can only very crudely be likened to the gas of electrons envisaged by the Drude model. Believing the analysis to be of limited value, we forego any presentation of results in this area.

Below $T_{c}$ there is a shift in the spectral weight from the mid-IR to the low frequency region. This low frequency Drude-like weight increases further with decreasing temperature and though it somewhat screens the phonon modes, they remain evident, even at the lowest temperature displayed. The broad $8000 \mathrm{~cm}^{-1}(1 \mathrm{eV})$ peak seems to transfer some of its weight into the $6000 \mathrm{~cm}^{-1}(0.7 \mathrm{eV})$ peak, very prominent in the $190 \mathrm{~K}$ spectrum, and the rest to the low energy Drude peak. The weight and energy of the $6000 \mathrm{~cm}^{-1}$ peak has decreased at $160 \mathrm{~K}$ as the Drude weight further increases, and at lower temperatures there appears to be a clear division of weight: increasing below and decreasing above about $4000 \mathrm{~cm}^{-1}$, or $0.5 \mathrm{eV}$. The temperature dependence of the weight of this Drude-like feature is investigated in the following subsection.

\section{Sum rules and carrier dynamics}

Because charge cannot be created or destroyed, the integral sum of the conductivity $\int \sigma_{1}(\omega) d \omega$, gives a way of identifying which spectral regions the charge carriers are accumulating in. It also enables the isolation of temperature-dependent features from a background that can often obscure these. The effective number of carriers below selected cutoff energies can be found by use of equation 2.58 , to which the conductivity sum rule is an integral part. In this expression, $V$, the volume of the unit cell, was calculated from reported temperature-dependent structural data[66, 67] on $\mathrm{La}_{0.75} \mathrm{Ca}_{0.25} \mathrm{MnO}_{3}$. This sample lies in the same structural phase of the $x=0.265$ substituted material, and its structural parameters are thus representative in magnitude and temperature dependence. 
Figure $5.5^{1}$ shows $N_{\text {eff }}(\omega, T)$ for $\mathrm{La}_{0.735} \mathrm{Ca}_{0.265} \mathrm{MnO}_{3}$ in the frequency range of 100 to $45000 \mathrm{~cm}^{-1}$. We note, first of all, for the $295 \mathrm{~K}$ and $255 \mathrm{~K}$ spectra, that the oscillator strength at the low frequency end accumulates in distinct steps, forming three plateau regions at the phonon energies. By contrast, the Drude-like peak for the $T<T_{c}$ spectra overwhelms the increase in oscillator strength contributed by the phonons for the material in its metallic-like phase.

The band at $6000 \mathrm{~cm}^{-1}$ shows a very significant increase in the carriers for the $295 \mathrm{~K}$ and $255 \mathrm{~K}$ spectra. This increase is notable in the $190 \mathrm{~K}$ spectrum, but for the $160 \mathrm{~K}, 130 \mathrm{~K}$ and $75 \mathrm{~K}$ spectra, the band is difficult to distinguish from the large Drude-like band whose oscillator strength extends from 100 to $25000 \mathrm{~cm}^{-1}$.

A notable feature of the curves is that the effective number of carriers for all of the different temperatures coincides at about $25000 \mathrm{~cm}^{-1}$. This indicates that the transfer of oscillator strength is restricted to the energy bands for $\omega<25000 \mathrm{~cm}^{-1}$, that is, that the transfer of carriers with temperature is occuring within the region of our data, and, more specifically, from the 6000 $\mathrm{cm}^{-1}$ band at high temperature to the low energy Drude-like band.

A close inspection of the spectra in Fig. 5.4 shows that there is a crossover energy below which the Drude weight increases with decreasing temperature and above which the spectral weight decreases as temperature decreases. This occurs at $\omega_{c} \approx 4000 \mathrm{~cm}^{-1}(0.5 \mathrm{eV})$. Fig. 5.6 displays $N_{\text {eff }}(\omega, T)$ for $\mathrm{La}_{0.735} \mathrm{Ca}_{0.265} \mathrm{MnO}_{3}$ calculated to $0.5 \mathrm{eV}$. The temperature dependence of $N_{\text {eff }}$ does not change significantly with energy cutoffs $\pm 0.05 \mathrm{eV}$.

As detailed previously, correlation between $\left(M(T) / M_{s}\right)^{2}$ and $N_{\text {eff }}$ is expected in the simple Double Exchange picture[68]. However, we find that $N_{\text {eff }}(T, 0.5 \mathrm{eV})$ is not proportional to our measurement of the square of the normalised magnetisation, $\left(M(T) / M_{s}\right)^{2}$, (where $M(T)$ is the magnetisation and $M_{s}$ is the saturated ferromagnetic magnetisation as $T \rightarrow 0$ ), shown as the inset to Fig. 5.6. The magnetisation shows a very sharp transition with temperature, as expected for a long-range electronic correlation, whereas the increase of (the effective number of) carriers with decreasing temperature is

\footnotetext{
${ }^{1}$ This analysis assumes that the effective mass is the same as the bare optical mass.
} 


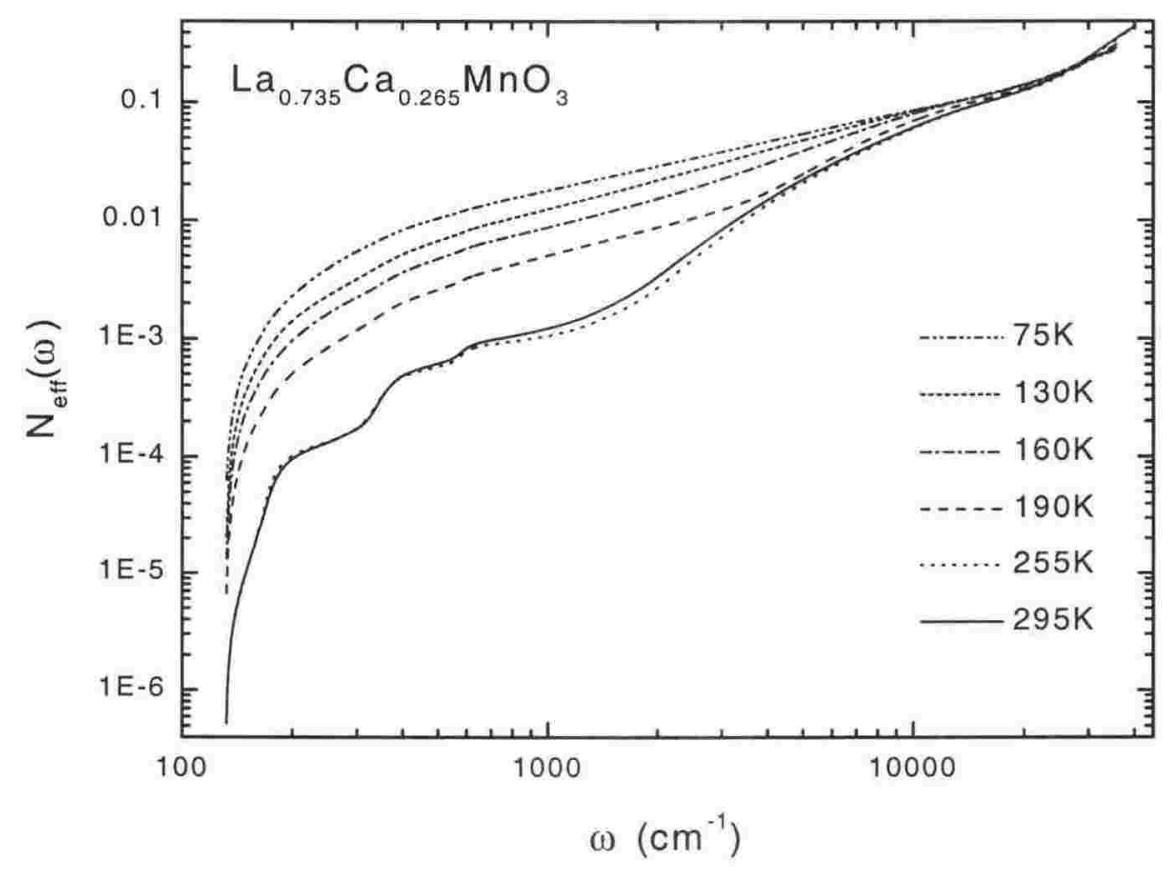

Figure 5.5: Effective number of carriers, $\mathrm{N}_{\text {eff }}(\omega)$, for the $\mathrm{La}_{0.735} \mathrm{Ca}_{0.265} \mathrm{MnO}_{3}$ sample found from the sum of $\sigma_{1, \kappa \kappa}(\omega, T)$ to $\omega$.

much more gradual. Our result implies that a Double Exchange model alone can not explain the spectral measurements.

Another way of expressing the Double Exchange picture is to focus on the energy region which is depleted as temperature decreases and examine how carriers are removed to participate in the low energy Drude-like band[68]. By using this analysis it has been found, in an absorption[31] study and a reflectance[34] study (both on $\mathrm{La}_{1-x} \mathrm{Sr}_{x} \mathrm{MnO}_{3}$ ), that the IR spectral weight associated with the conducting carriers is transferred from the higher energy transitions at $3.2 \mathrm{eV}$ and $5 \mathrm{eV}[34]$, in obeyance with the scaling relation $1-\left(M(T) / M_{s}\right)^{2}$. This implies that the Double Exchange picture alone is sufficient to explain the loss of carriers at high energy. Similar analysis carries large uncertainties for the present dataset, as the lesser skindepth for the higher frequency region probes proportionally more of the damaged surface 


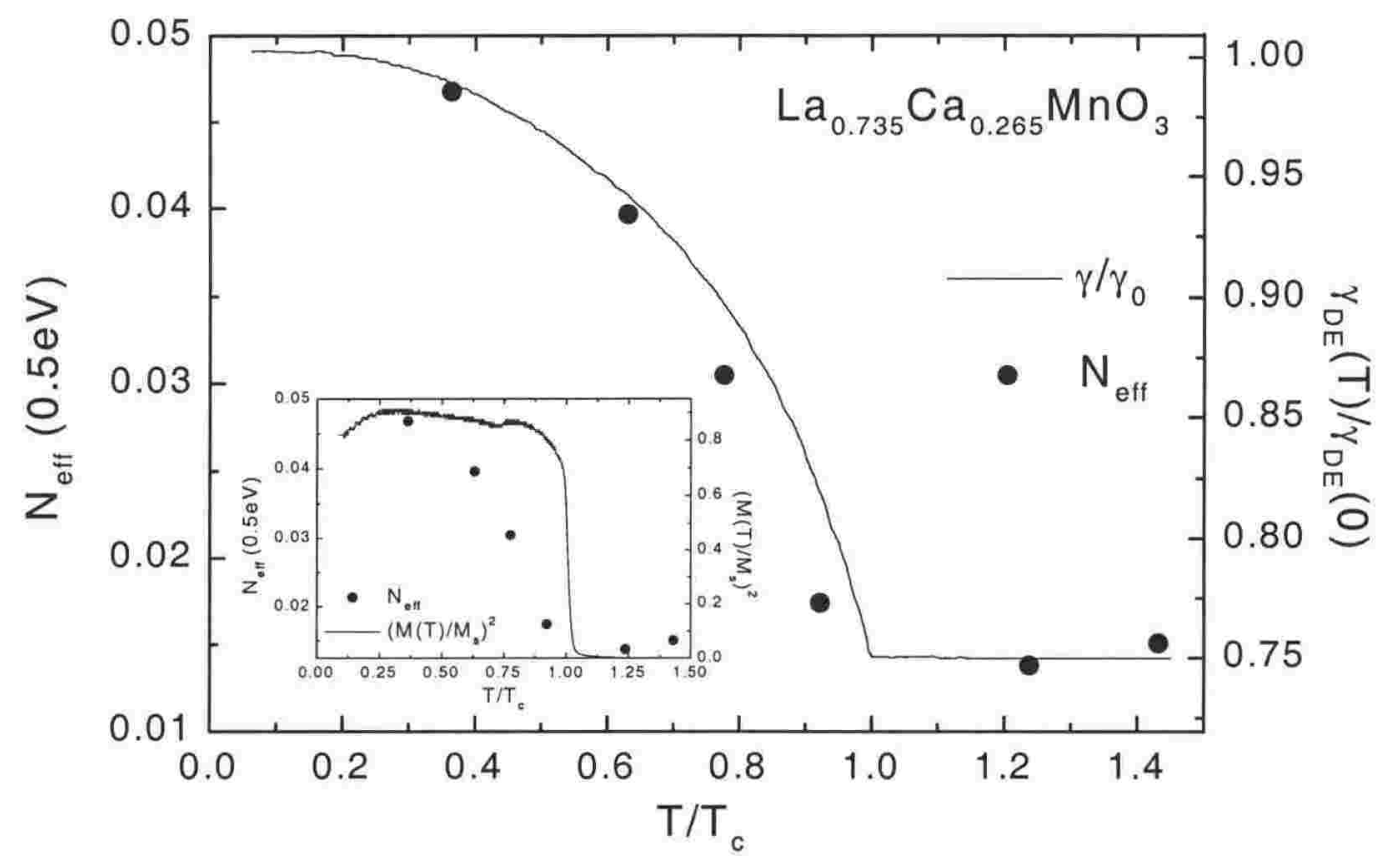

Figure 5.6: Effective number of carriers, $N_{\text {eff }}(0.5 \mathrm{eV})$, for the $\mathrm{La}_{0.735} \mathrm{Ca}_{0.265} \mathrm{MnO}_{3}$ sample, found from the sum of the conductivity to 0.5 $\mathrm{eV}$ and contrasted with the temperature-dependent Double Exchange bandwidth $\gamma / \gamma_{0}$.

layer than the bulk, so we have not attempted it. Absorption techniques[31] are far less sensitive to surface effects, and a cleaved surface is far more likely to reflect the properties of the bulk, thus the spectra in the short wavelength region for absorption and cleaved surface reflectivity studies are more reliable than surface probe techniques in which the surface quality is unknown.

Our $N_{\text {eff }}(T)$ does, however, scale rather well with a parameter which also incorporates Jahn-Teller coupling in the Double Exchange model. This is the temperature-dependent Double Exchange bandwidth, $\gamma_{D E}(T) / \gamma_{D E}(0)$, predicted by Kubo and Ohata[11] and illustrated as a solid line in Fig. 5.6. By contrast with $\left(M(T) / M_{s}\right)^{2}$, the rise of $\gamma_{D E}(T) / \gamma_{D E}(0)$ with decreasing temperature is much more gradual and tracks $N_{\text {eff }}(0.5 \mathrm{eV})$ rather well. The reason for this good agreement, also seen in other optical studies[32, 65, 69], 


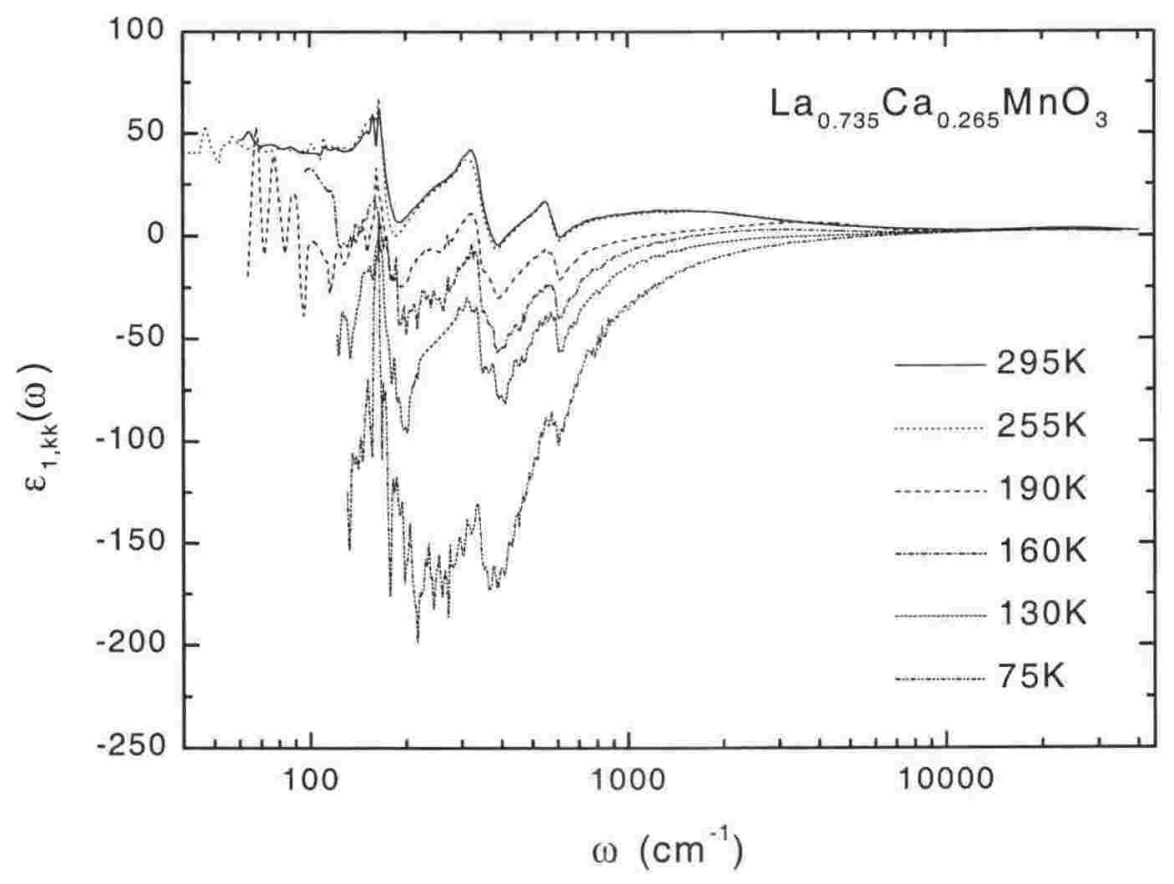

Figure 5.7: $\epsilon_{1}(\omega)$ found from a Kramers-Kronig transform of $R(\omega)$ for the annealed $\mathrm{La}_{0.735} \mathrm{Ca}_{0.265} \mathrm{MnO}_{3}$ sample.

has been discussed by Kim and coworkers[69] in terms of incorporation of Jahn-Teller coupling in the Double Exchange model, whose Hamiltonian was studied by Röder and coworkers[70].

In the previous chapter we demonstrated that the quantitative interpretation of results of any optical measurement is not independent of surface treatments. The surface treatments, adopted in the three optical studies which applied the temperature-dependent Double Exchange bandwidth analysis, were annealing of a single crystal[32], annealing of polycrystals[69], and polishing of a polycrystal[65]. Given the uncertainty associated with the optical studies of polished and annealed materials, a precise picture of the electron dynamics in the $\mathrm{La}_{1-x} \mathrm{Ca}_{x} \mathrm{MnO}_{3}$ system is probably yet to emerge. 
$\epsilon_{1, \kappa \kappa}(\omega, T)$

For completeness, we include Fig. 5.7, which shows $\epsilon_{1, \kappa \kappa}(\omega, T)$ for the $\mathrm{La}_{0.735} \mathrm{Ca}_{0.265} \mathrm{MnO}_{3}$ sample. For $T>T_{c}, \epsilon_{1, \kappa \kappa}(\omega)$ has a positive DC value of about 50 . The low energy region is dominated by the phonon behaviour and, as the energy increases further, the spectrum converges with the spectra in the $T<T_{c}$ phase to the expected high frequency value of $\epsilon_{1}=1$. As the temperature decreases away from $T_{c}$, the low energy $\epsilon_{1, \kappa \kappa}$ becomes more strongly negative, yet still featuring quite prominent phonon signatures. These are less obvious in the $75 \mathrm{~K}$ spectrum, which shows a definite trend to negative values with decreasing frequency for $\omega>400 \mathrm{~cm}^{-1}$. Below this frequency the spectrum is dominated by noise.

As the inductive part of the conductivity, $\epsilon_{1, \kappa \kappa}(\omega, T)$ completes the full description of the response function and clearly shows an increasing metallic behaviour with decreasing temperature, although the previous section has highlighted that the dynamics have yet to be completely characterised.

\subsection{Layered $\mathrm{La}_{2-2 x} \mathrm{Sr}_{1+2 x} \mathrm{Mn}_{2} \mathrm{O}_{7}$.}

The $n=2$ members of the Ruddlesden-Popper structural series of manganites, with their highly anisotropic structure, display intriguing physical variations from the $n=\infty$ pseudocubic manganites. Attention has thus recently turned to the properties of the layered materials. Optical measurements of $x=0.4$ hole substituted $\mathrm{La}_{1.2} \mathrm{Sr}_{1.8} \mathrm{Mn}_{2} \mathrm{O}_{7}$ are presented in this section of the chapter.

\subsubsection{Magnetisation of $\mathrm{La}_{1.2} \mathrm{Sr}_{1.8} \mathrm{Mn}_{2} \mathrm{O}_{7}$.}

To set the scene for the discussion of the electronic and optical properties of this material, magnetisation measurements are first presented. These were acquired with a Vibrating Sample Magnetometer (VSM) on the sample used in the optical experiment. Once in the VSM cryostat, the $a b$ plane and $c$-axis directions of the sample were found by rotating through $360^{\circ}$ and measuring the angular dependence of the sample's magnetic susceptibility, $\chi$. A sinusoidal dependence was found. From literature on the magnetic proper- 
ties of $\mathrm{La}_{1.2} \mathrm{Sr}_{1.8} \mathrm{Mn}_{2} \mathrm{O}_{7}[6]$ it is known that the peak in $\chi$ corresponds to the $a b$ plane and the minimum $90^{\circ}$ either side of this is found when the $c$-axis is being probed. The magnetisation measurements were made in a 5 hour temperature sweep whilst the sample was warming. Fig. 5.8(a) displays the temperature-dependent magnetisation $M(T)$ measured for the two orientations of the crystal and in two different field strengths.
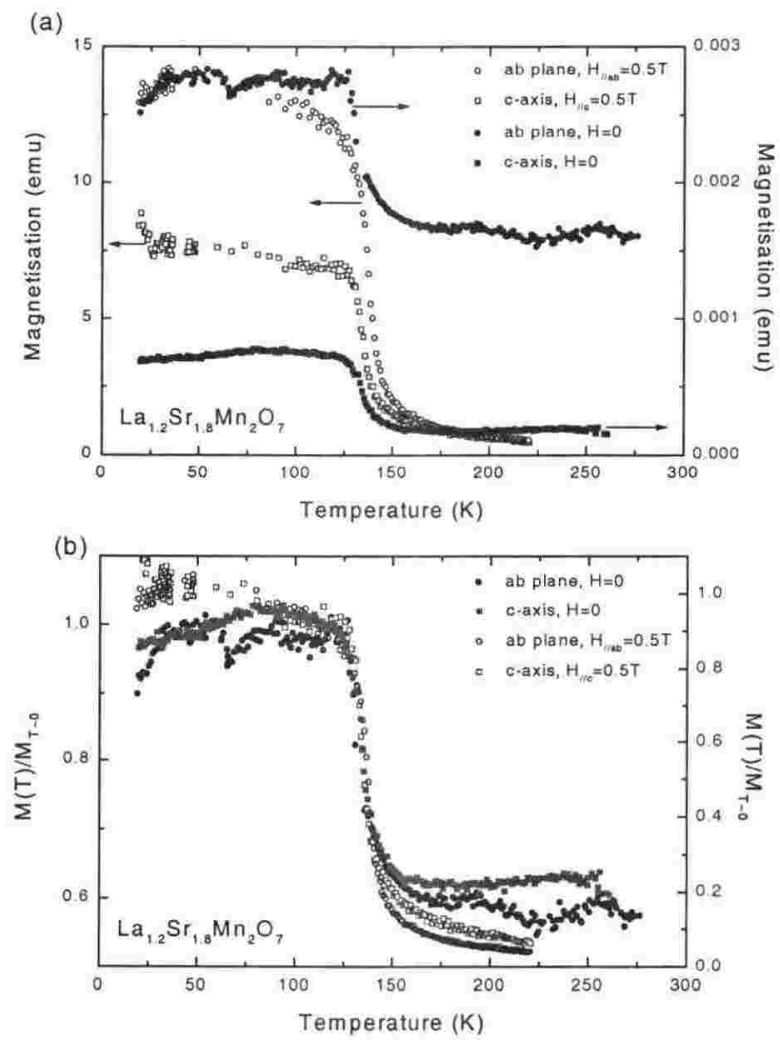

Figure 5.8: Panel (a): Magnetisation measured along the $a b$ plane (circles) and $c$-axis (squares) of $\mathrm{La}_{1.2} \mathrm{Sr}_{1.8} \mathrm{Mn}_{2} \mathrm{O}_{7}$. Filled and open shapes denote the magnetisation response to $H=0 \mathrm{~T}$ and $H=0.5 \mathrm{~T}$ respectively. Panel (b): Scaled magnetisation curves, demonstrating the ferromagnetic ordering temperature for the $a b$ plane and $c$-axis at $125 \mathrm{~K}$.

As can be seen by the open shapes in Fig. 5.8, denoting the magnetic response in a field of $0.5 \mathrm{Tesla}$, the $a b$ plane and $c$-axis of layered $\mathrm{La}_{1.2} \mathrm{Sr}_{1.8} \mathrm{Mn}_{2} \mathrm{O}_{7}$ at $20 \mathrm{~K}$ have an induced magnetisation of approximately 13 
and 8 emu respectively. The sample remains magnetised to about $100 \mathrm{~K}$ as the temperature is increased. As the temperature further increases through a narrow temperature band from $T_{c} \approx 125 \mathrm{~K}$ to $150 \mathrm{~K}$ the spins become randomised and the magnetisation decreases to near zero. The near-zero temperature magnetisation $M(T \rightarrow 0)$ of the $c$-axis is about $60 \%$ that of the $a b$ plane. Similarly, for the spontaneous magnetisation measured in zero field, denoted by solid squares and circles for the $c$-axis and $a b$ plane responses respectively, as the temperature is warmed through the same narrow band, the magnetic order along the $c$-axis is destroyed. Along the $a b$ plane this order also decreases, but settles at a magnetisation level in its paramagnetic state of $\approx 1.5$ memu. This is twice as great as the low-temperature saturation magnetisation of the $c$-axis and several times greater than the paramagnetic state level of the $c$-axis magnetisation. Nonetheless, as seen in panel (b) of Fig. 5.8, when these curves are normalised to their low-temperature value, they all collapse onto an identical curve showing ferromagnetic order below the transition temperature band between $125 \mathrm{~K}$ and $150 \mathrm{~K}$. Note that, in panel (b), the left $\mathrm{y}$-axis applies to the $a b$ plane response in ambient field, and the right $\mathrm{y}$-axis applies to the other 3 curves.

The resistivity curves of Fig. 5.1 also show that the $a b$ plane of the material has an insulator $\rightarrow$ metallic-like transition at $125 \mathrm{~K}$. At all temperatures the $c$-axis resistivity is 2 orders of magnitude higher than the $a b$ plane resistivity, closely mapping the shape of the $a b$ plane curve. Both $c$-axis and $a b$ plane resistivity display the same sharp transition at $125 \mathrm{~K}$. Thus, ferromagnetic order and the sharp increase in conductivity occur at a coincident temperature, in accord with Double Exchange pictures applied to the pseudocubic manganites.

\subsection{2 $\sigma_{1}(\omega, T)$ for $\mathbf{L a}_{1.2} \mathrm{Sr}_{1.8} \mathrm{Mn}_{2} \mathrm{O}_{7}$.}

\section{Description of mid-IR $\sigma_{1}(\omega, T)$}

Figure 5.9 displays the temperature-dependent $a b$ plane and room temperature $c$-axis conductivity in the energy range of $0 \leq \omega \leq 5000 \mathrm{~cm}^{-1}$. Plotted as open shapes on the $y$-axis are the DC conductivity values from the 
temperature-dependent $a b$ plane resistivity measurements[63]. We discuss the spectra methodically, in order of decreasing temperature, starting with the $c$-axis spectrum at $295 \mathrm{~K}$. This displays three quite broad phonon features (displayed separately and discussed later), a gap-like feature between $600 \mathrm{~cm}^{-1}$ and $800 \mathrm{~cm}^{-1}$, and then a gentle rise in the mid-IR conductivity value. The far-IR conductivity is very low, consistent with the high DC resistivity of this material. The mid-IR $\tilde{\sigma}(\omega, T)$ is not measured for the $c$-axis of this sample, but the temperature-dependence of the $c$-axis conductivity has been shown $[26,63]$ to be very slight in the mid-IR and visible regions.

The $a b$ plane conductivity at $250 \mathrm{~K}$ also displays three prominent phonon features in the far-IR. The reader is referred on a few pages for a blowup of this region in Fig. 5.10(a). These three in-plane modes at $\omega \approx 160$ $\mathrm{cm}^{-1}, 350 \mathrm{~cm}^{-1}$, and $610 \mathrm{~cm}^{-1}$ (refer to Table 5.2) will be, in like manner to the pseudocubic material, respectively referred to as the low-energy, midenergy, and high-energy modes of vibration. It is interesting to note that Lee and coworkers[71] have assigned the high-energy mode to the "stretching" vibration by analogy with the pseuocubic manganite assignments[22]. In the $250 \mathrm{~K}$ spectrum, the conductivity rises to form the low energy side of a broad mid-IR absorption feature which has been previously observed to peak at 1.2 $\mathrm{eV}\left(10000 \mathrm{~cm}^{-1}\right)$ for $T>T_{c}[26,63,71]$. At $200 \mathrm{~K}$ the spectral weight in the energy range of $650-2000 \mathrm{~cm}^{-1}$ decreases slightly, implying a narrowing of the $1.2 \mathrm{eV}\left(10000 \mathrm{~cm}^{-1}\right)$ band, which has been previously noted[26, 63, 71]. The $200 \mathrm{~K}$ and $250 \mathrm{~K}$ spectra are otherwise very similar. As the temperature drops further to $150 \mathrm{~K}$, the conductivity level in the frequency range of 700-5000 $\mathrm{cm}^{-1}$ increases, but with little difference in shape from the other $T>T_{c}$ spectra. There is little discernible temperature-dependence in the phonon modes above $T_{c}$.

At $125 \mathrm{~K}$, the material begins to move through its paramagnetic insulator $\rightarrow$ ferromagnetic metal transition and spectral changes become more dramatic. In the far-IR, the phonon modes, though of comparable strength to the $T>T_{c}$ spectra, are superimposed on a broad far-IR absorption feature, which is rounded, peaking at $300 \mathrm{~cm}^{-1}$. The high-energy mode displays an increase in frequency and significant broadening. The spectral weight 


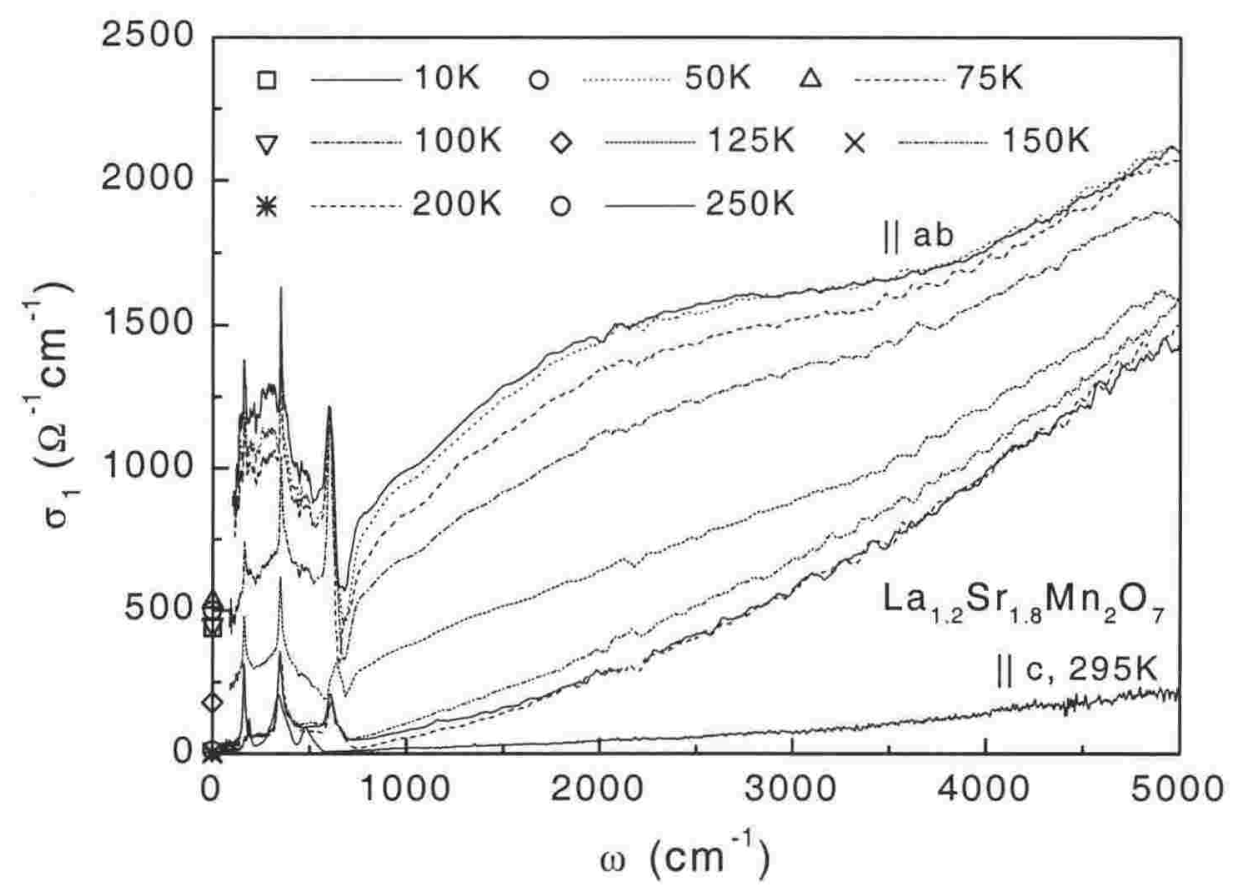

Figure 5.9: Temperature-dependent $\sigma_{1}(\omega)$ spectra for $\mathrm{La}_{1.2} \mathrm{Sr}_{1.8} \mathrm{Mn}_{2} \mathrm{O}_{7}$ to $5000 \mathrm{~cm}^{-1}$. Note that the temperature increases from the top line to the lowest line. DC conductivity values, at the temperatures indicated in the legend, are plotted as shapes on the $y$-axis.

has increased in the entire mid-IR range measured, with a distinct inflexion point at $\omega \approx 3500 \mathrm{~cm}^{-1}$, as though demarquing a cut-off between different absorptions. For energies greater than this the gradient of the rising conductivity slope becomes steeper. Moving below $T_{c}$, at $100 \mathrm{~K}$ the far-IR absorption background more than doubles in magnitude from its $125 \mathrm{~K}$ conductivity value, remaining rounded in shape with no Drude-like turn up at low frequency. The high-energy mode shows a marked increase in weight at $100 \mathrm{~K}$ over and above the increase in far-IR background weight. This change occurs very abruptly between $125 \mathrm{~K}$ and $100 \mathrm{~K}$. The frequency of the mode, however, appears to have decreased back to its $T>T_{c}$ value.

The spectra at $100 \mathrm{~K}$ and below show a pronounced gap-like feature at $\omega \approx 700 \mathrm{~cm}^{-1}$, suggesting quite a distinct separation of the far-IR and mid- 
IR absorption bands. As the conductivity of the mid-IR part continues to grow with decreasing temperature, a peak centred at $\omega \approx 2000 \mathrm{~cm}^{-1}$ begins to push up from the low energy flank of the broader mid-IR band. The shapes of the $75 \mathrm{~K}, 50 \mathrm{~K}$ and $10 \mathrm{~K}$ spectra are largely similar, though the conductivity continues to grow in this temperature range as temperature decreases. The broad far-IR absorption feature peaks at $\omega \approx 300 \mathrm{~cm}^{-1}$, or a little lower in energy than the mid-energy phonon, with a magnitude of $\sigma_{1} \approx 1250 \Omega^{-1} \mathrm{~cm}^{-1}$. Unusually, after its abrupt increase in strength at $100 \mathrm{~K}$, the conductivity peak of the high-energy phonon remains the same as the temperature is decreased to $10 \mathrm{~K}$, the background in a sense "catching up" with this Mn-O bond stretching absorption. The shape of this phonon is remarkably reminiscent of a Fano-like absorption, an asymmetric peak resulting from lattice-electron continuum interaction. However, it is also in a region of high uncertainty due to the reduction process. We, thus, resist any temptation to fit the feature to a Fano resonance. By contrast with the material's pseudocubic counterpart, $\mathrm{La}_{1-x} \mathrm{Sr}_{x} \mathrm{MnO}_{3}$, entering its metallic phase $[25,30,34,61,72]$ the phonons persist in strength despite the rising background as the temperature decreases. Screening is clearly less important for the layered materials due to a reduced carrier density.

\section{Description of far-IR $\sigma_{1}(\omega, T)$}

Figure 5.10 (a) displays temperature-dependent $a b$ plane conductivity spectra in the far-IR. On the $y$-axis are plotted DC conductivity values from reported DC resistivity measurements. Assuming a gradual continuation of the rounded background, discussed in the last subsection, to a zero frequency value for each temperature, it appears that the ellipsometric optical constants, extrapolated to zero frequency, are in agreement with the reported DC conductivity values. The shape of the conductivity background for $T<T_{c}$, rounded and increasing in spectral weight and peak magnitude as temperature decreases, shows no Drude-like turn up as would be expected for a metallic material. For $T<T_{c}$, the shape of the low frequency absorption indicates that the Drude model alone is incompatible with the $\mathrm{La}_{1.2} \mathrm{Sr}_{1.8} \mathrm{Mn}_{2} \mathrm{O}_{7}$ metallicity. 


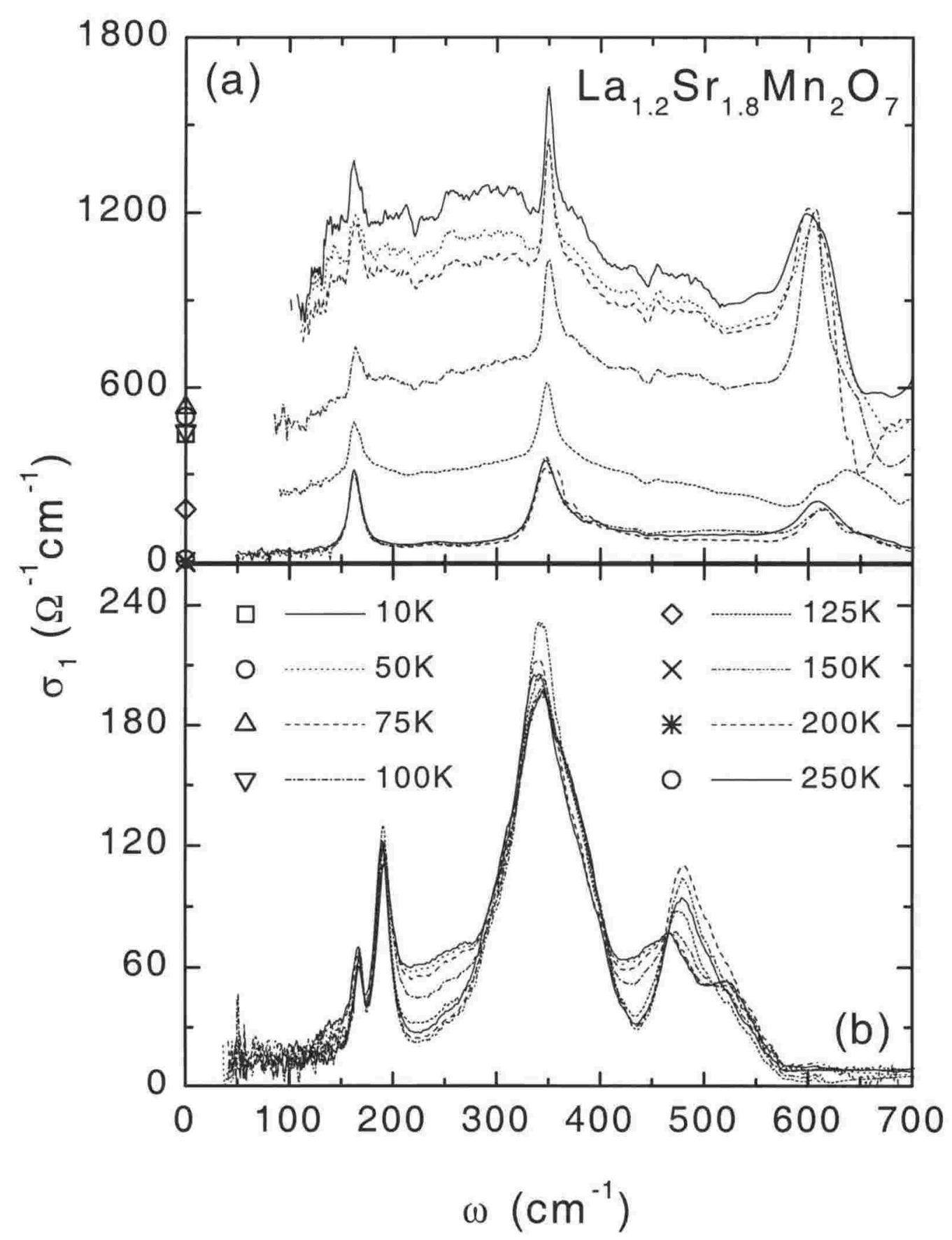

Figure 5.10: Temperature-dependent $\sigma_{1}(\omega)$ (a) $a b$ plane and (b) $c$-axis spectra for $\mathrm{La}_{1.2} \mathrm{Sr}_{1.8} \mathrm{Mn}_{2} \mathrm{O}_{7}$ to $700 \mathrm{~cm}^{-1}$. Shapes on the $y$-axis denote DC conductivities, from temperature-dependent resistivity measurements, at the temperatures indicated in the legend. 
In their optical study of $\mathrm{La}_{1.2} \mathrm{Sr}_{1.8} \mathrm{Mn}_{2} \mathrm{O}_{7}$, Ishikawa and coworkers[63] fit their data to a Drude model for all temperatures and present a comparison between predicted and measured DC conductivities. They find quite good agreement for $T>100 \mathrm{~K}$, that is, in the insulating phase, but further into the metallic phase observe the Drude DC conductivity is at least 2.5 and up to 4 times greater than the measured DC conductivity. Both that study and this work clearly indicate that a Drude description must either be seriously revised or thrown out the window before being applied to the layered manganites.

We leave the already amply discussed $a b$ plane conductivity now, to discuss the far-IR $c$-axis conductivity shown in figure 5.10 (b). There is some striking temperature-dependence in this spectrum which has not been previously investigated. At $250 \mathrm{~K}$ there are four main peaks in the spectrum, at $\omega \approx 165 \mathrm{~cm}^{-1}, 190 \mathrm{~cm}^{-1}, 340 \mathrm{~cm}^{-1}$ and $480 \mathrm{~cm}^{-1}$ (see actual values in Table 5.2). The doublet of lowest energy modes are probably oscillations associated with the heavy rare-earth ion. For convenience of further description we refer to the doublet collectively as the low-energy mode.

With an increase of temperature above $T_{c}$ the low-energy and mid-energy modes show a well behaved anharmonic broadening and softening. The highenergy phonon at $480 \mathrm{~cm}^{-1}$ displays unusual temperature-dependence. An increase in spectral weight accompanies the decrease from $250 \mathrm{~K}$ to $200 \mathrm{~K}$. The mode weight then decreases with the temperature decrease to $125 \mathrm{~K}$. At $125 \mathrm{~K}$ the mode begins to show a growth at its higher energy side, which becomes more pronounced with decreasing temperature. At $75 \mathrm{~K}$ and below there is a clearly discernible split to two phonons.

Another interesting temperature-dependent feature is the increase of spectral weight, or what we term a "filling", either side of the $340 \mathrm{~cm}^{-1}$, midenergy phonon. This shows an onset at $125 \mathrm{~K}$, with the most dramatic increase occuring between $125 \mathrm{~K}$ and $100 \mathrm{~K}$. The absorption increases further with each step down in temperature. The absorption begins to saturate at $50 \mathrm{~K}$, reaching an apparent maximum at $10 \mathrm{~K}$. There are some points to note about these features. First, the "fillings" appear to be isolated to the interphonon region. Apart from an abrupt increase in the mid-energy phonon peak height at $150 \mathrm{~K}$, the strength of the doublet and the mid-energy mode 
remain virtually unchanged. The "filling" effect does not appear to be affecting the shape or strength of any of the three lowest energy phonons. Secondly, no "filling" is observed between the phonons of the low-energy doublet. This is in spite of the fact that the saturated filling level at $220 \mathrm{~cm}^{-1}$ is almost as great as the peak conductivity of the $165 \mathrm{~cm}^{-1}$ member of the doublet. Finally, no absorption increases are observed for $\omega<150 \mathrm{~cm}^{-1}$ and $\omega>570$ $\mathrm{cm}^{-1}$.

Contrasting the positions of the modes in Fig. 5.10 reveals that the energy of the $a b$ plane low-energy oscillation matches that of the lowest energy member of the $c$-axis doublet, at $165 \mathrm{~cm}^{-1}$. The higher energy member sits at $190 \mathrm{~cm}^{-1}$. An extra peak near the energy of this mode is seen in the pseudocubic $\mathrm{La}_{1-x} \mathrm{Sr}_{x} \mathrm{MnO}_{3}$ at substitution levels of $0.15,0.16,0.18$ and 0.2 in [72] and a doubling of the "rare earth" mode at $x=0.175[30,61]$. However, the doubling effect at these substitution levels is washed out by the increase of Drude weight as the temperature decreases into the materials' metallic phase. A doublet is not observed for the corresponding substitution level of $x=0.4$ in the pseudocubic material. Its appearance in the optical spectra is undoubtedly due to the radically different symmetry of the $n=2$ lattice in the $c$-axis direction compared to the $n=\infty$ crystal. However, without lattice dynamics calculations for this structure, we are at this time unable to assign the extra phonon to the vibration responsible for it.

The positions of the mid-energy modes appear at much the same frequency, with the $c$-axis mode softer by $5 \mathrm{~cm}^{-1}$. The position of the $a b$ plane and $c$-axis high-energy modes however, at $610 \mathrm{~cm}^{-1}$ and $480 \mathrm{~cm}^{-1}$, are very far removed from each other. However at this stage it can merely be asserted that these shifts exist. It is tempting to blindly assign the modes to the "rare earth", "bending" and "stretching" phonons, in order to facilitate a deeper discussion. However these mode assignments strictly apply only to the pseudocubic materials. Which atoms are actually contributing to the modes seen in $\mathrm{La}_{1.2} \mathrm{Sr}_{1.8} \mathrm{Mn}_{2} \mathrm{O}_{7}$ is a question only answerable by lattice dynamics calculations which, as mentioned, are not yet available for the layered manganites.

In Table 5.2, we display a comparison of the phonons for all of the man- 


\begin{tabular}{|r||c|c|c|c|c|c|c|c|}
\hline \multicolumn{2}{|c||}{ Sample } & \multicolumn{2}{c|}{ Lo-energy } & \multicolumn{2}{c|}{ Lo-energy $(c)$} & \multicolumn{2}{c|}{ Mid-energy } & \multicolumn{2}{c|}{ Hi-energy } \\
\hline \hline $\mathrm{La}_{1-x} \mathrm{Ca}_{x} \mathrm{MnO}_{3}$ & $295 \mathrm{~K}$ & $75 \mathrm{~K}$ & & & $295 \mathrm{~K}$ & $75 \mathrm{~K}$ & $295 \mathrm{~K}$ & $75 \mathrm{~K}$ \\
\cline { 2 - 9 }$x=0.1$ & 162 & - & & & 345 & - & 577 & - \\
$x=0.265$ & 173 & 176 & & & 350 & 343 & 583 & 600 \\
\hline \hline $\mathrm{La}_{1,2} \mathrm{Sr}_{1.8} \mathrm{Mn}_{2} \mathrm{O}_{7}$ & $250 \mathrm{~K}$ & $10 \mathrm{~K}$ & $250 \mathrm{~K}$ & $10 \mathrm{~K}$ & $250 \mathrm{~K}$ & $10 \mathrm{~K}$ & $250 \mathrm{~K}$ & $10 \mathrm{~K}$ \\
\cline { 2 - 9 }$a b$ plane & 163 & 162 & - & - & 348 & 350 & 612 & 600 \\
$c$-axis & 167 & 166 & 189 & 192 & 339 & 344 & 480 & - \\
\hline \hline $\mathrm{PrSr}_{2} \mathrm{Mn}_{2} \mathrm{O}_{7}$ & $295 \mathrm{~K}$ & $10 \mathrm{~K}$ & $295 \mathrm{~K}$ & $10 \mathrm{~K}$ & $295 \mathrm{~K}$ & $10 \mathrm{~K}$ & $295 \mathrm{~K}$ & $10 \mathrm{~K}$ \\
\cline { 2 - 9 }$a b$ plane & 165 & 164 & - & - & 348 & 352 & 610 & 616 \\
$c$-axis & 170 & 174 & 198 & 202 & 342 & 346 & 495 & 503 \\
\hline
\end{tabular}

Table 5.2: Phonon frequencies in $\mathrm{cm}^{-1}\left( \pm 1 \mathrm{~cm}^{-1}\right)$ of the manganite compounds investigated in this study. The phonons are given for the high $(295 \mathrm{~K}$ or $250 \mathrm{~K})$ and low $(75 \mathrm{~K}$ or $10 \mathrm{~K})$ extremes of temperature for which measurements were made.

ganite samples measured, including the principal modes of the yet-to-be discussed $\mathrm{PrSr}_{2} \mathrm{Mn}_{2} \mathrm{O}_{7}$. For each sample the phonon frequency for each of the high and low extremes of temperature has been recorded.

\section{Frustrated Drude response}

It is certainly not unusual that metallic materials can exhibit such an odd Drude response, as observed for the $a b$ plane of $\mathrm{La}_{1.2} \mathrm{Sr}_{1.8} \mathrm{Mn}_{2} \mathrm{O}_{7}$. There are various localisation models which have been devised to explain electronic behaviour in disordered systems. These systems include conducting polymers[73], high $T_{c}$ superconductors[74], and even so-called simple metals[75]. We will not discuss these here, except to mention the Localisation Modified Drude (LMD) model, which has been applied to other manganites. The LMD model follows from Anderson localisation and, in contrast with the Mott insulator[76], is due to disorder in the system. Anderson localisation has been suggested as an explanation for the large and broad far-IR $\sigma_{1}$ peaks observed in the pseudo-cubic manganites, $\mathrm{La}_{0.7-y} \mathrm{Pr}_{y} \mathrm{Ca}_{0.3} \mathrm{MnO}_{3}(y=0.13,0.4$, 0.5)[65]. All three of these spectral sets display a peak increase in $\sigma_{1}$ of the order of $10^{3} \Omega^{-1} \mathrm{~cm}^{-1}$ with the decrease in temperature from $290 \mathrm{~K}$ to $20 \mathrm{~K}$. Given that these conductivity increases are of comparable magnitude to that 
observed in $\mathrm{La}_{1.2} \mathrm{Sr}_{1.8} \mathrm{Mn}_{2} \mathrm{O}_{7}$ between 100 and $700 \mathrm{~cm}^{-1}$, it appears possible that Anderson localisation could explain the charge dynamics in the layered manganites. While Mitchell and coworkers[77] assert that $\mathrm{La}_{1.2} \mathrm{Sr}_{1.8} \mathrm{Mn}_{2} \mathrm{O}_{7}$ does in fact become more disordered in structure with decreasing temperature, and disorder effects have been predicted as possibly important in manganites $[78,79]$, at this point we can only say that the data is consistent by analogy, with the LMD model of disorder, and hence Anderson localisation.

Having meticulously described the data, we turn now to an analysis. In the next section, the broad absorption bands in both the $a b$ plane and the $c$-axis spectra are investigated. The subsequent section will address the issue of the in-plane carrier dynamics of the material, and how to explain the nonDrude low frequency absorption. A final section will deal with the striking temperature-dependence seen in the high-energy mode of both $a b$ plane and $c$-axis.

\subsubsection{Spectral weight changes}

In this subsection we investigate temperature-dependent spectral weight changes observed in $\mathrm{La}_{1.2} \mathrm{Sr}_{1.8} \mathrm{Mn}_{2} \mathrm{O}_{7}$ spectra. We discuss the $a b$ plane response first, the $c$-axis response second, and finally present these together in a comparison.

\section{$a b$ plane}

Pictured in Fig. 5.11 are the temperature-dependent spectra of the $a b$ plane $\mathrm{La}_{1.2} \mathrm{Sr}_{1.8} \mathrm{Mn}_{2} \mathrm{O}_{7}$ with the $250 \mathrm{~K}$ spectrum subtracted off. This removes the "room temperature" component of the mid-IR $1.2 \mathrm{eV}\left(10000 \mathrm{~cm}^{-1}\right)$ peak, and enhances our view of the broad $2000 \mathrm{~cm}^{-1}(0.25 \mathrm{eV})$ peak considerably. The $0.25 \mathrm{eV}$ feature in this dataset has not been observed previously and is characterised by a dramatic increase of spectral weight with decreasing temperature. It also displays an apparent independence of its frequency position with temperature. Unlikely to be due to an electronic transition(consult ref. [?] for band structure information), features at this energy have been modelled by electron-phonon interactions[80]. It appears to be a different feature 


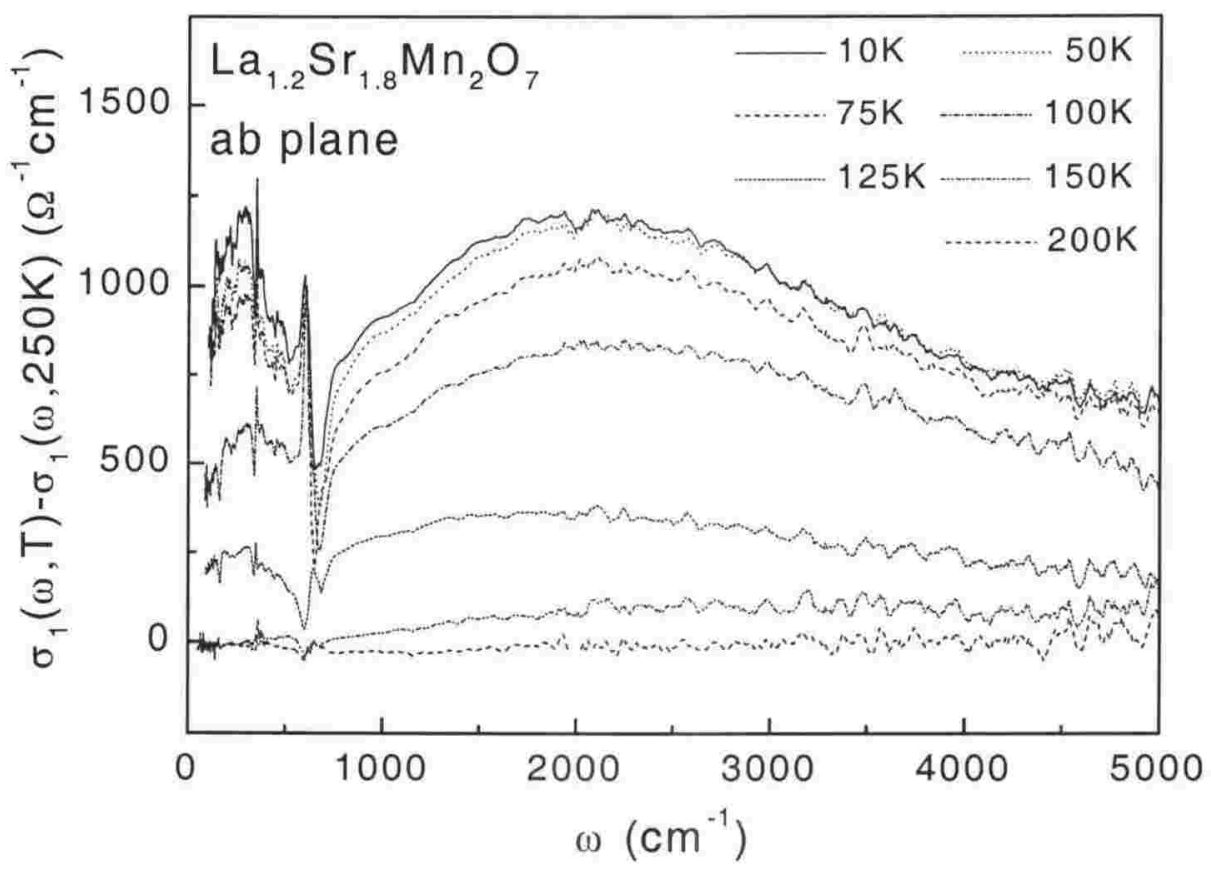

Figure 5.11: Temperature-dependent $\sigma_{1}(\omega, T)-\sigma_{1}(\omega, 250 \mathrm{~K})$ spectra for $a b$ plane $\mathrm{La}_{1.2} \mathrm{Sr}_{1.8} \mathrm{Mn}_{2} \mathrm{O}_{7}$.

to that modelled by Lee and coworkers[71], who found $\sigma_{1}(\omega)$ by normal incidence reflection for $\mathrm{La}_{1.2} \mathrm{Sr}_{1.8} \mathrm{Mn}_{2} \mathrm{O}_{7}$. They fit the $1.2 \mathrm{eV}\left(10000 \mathrm{~cm}^{-1}\right)$ band with 2 oscillators, a temperature-independent on-site $d$ - $d$ transition at 1.5 $\mathrm{eV}\left(12000 \mathrm{~cm}^{-1}\right)$ and a polaron absorption at around $0.6 \mathrm{eV}\left(5000 \mathrm{~cm}^{-1}\right)$. Their "polaron" has a temperature-dependent energy decrease from $0.75 \mathrm{eV}$ $\left(6000 \mathrm{~cm}^{-1}\right)$ at $295 \mathrm{~K}$ to $0.58 \mathrm{eV}\left(4700 \mathrm{~cm}^{-1}\right)$ at $10 \mathrm{~K}$. This occurs via an intermediate increase to $1 \mathrm{eV}\left(8000 \mathrm{~cm}^{-1}\right)$ at $T_{c}=126 \mathrm{~K}$. However, unlike the $0.25 \mathrm{eV}\left(2000 \mathrm{~cm}^{-1}\right)$ feature here, the spectral weight of their "polaron" is temperature-independent. The temperature-dependence of the spectral weight here is now investigated.

For ease of further description, the net spectral weight increase seen, for example, as the area underneath the curves in Fig. 5.11, is now defined as

$$
\varsigma(T)=\int_{\omega_{l}}^{\omega_{h}}\left(\sigma_{1}(T)-\sigma_{1}(250 K)\right) d \omega
$$


where $\omega_{l}$ and $\omega_{h}$ are the low and high frequency integration limits respectively. For the $a b$ plane, $\varsigma(T)$ was calculated in two different energy regions; between $\omega_{l}=0 \mathrm{~cm}^{-1}$ and $\omega_{h}=700 \mathrm{~cm}^{-1}$, incorporating the broad $300 \mathrm{~cm}^{-1}$ peak observed in the far-IR (phonon contributions were removed), and between $\omega_{l}=700 \mathrm{~cm}^{-1}$ and $\omega_{h}=4000 \mathrm{~cm}^{-1}$, encompassing the even broader 2000 $\mathrm{cm}^{-1}$ peak. The results of this analysis will be presented in a later subsection.

As an aside, we note that the display choice in Fig. 5.11 also highlights frequency changes in the phonon modes. These are signalled by the appearance of asymmetric features in spectra for which the mode has shifted from its $250 \mathrm{~K}$ position. The hardening in the high-energy mode at $125 \mathrm{~K}$, and resoftening for $T<T_{c}$, is evidenced in the asymmetric feature in the $125 \mathrm{~K}$ spectrum. By contrast, the mid-energy mode exhibits a hardening which sets in at $125 \mathrm{~K}$, but persists to the lowest temperatures. The low-energy mode shows no shifts in frequency, however its strength is observed to decrease at $125 \mathrm{~K}$ and $100 \mathrm{~K}$, as evidenced by a dip in $\sigma_{1}(T)-\sigma_{1}(250 \mathrm{~K})$ at this energy. It is restored to its $250 \mathrm{~K}$ weight at temperatures of $75 \mathrm{~K}$ and below.

$c$-axis

If the spectral weight being transferred to the $c$-axis "filling" features were due to being removed from the high-energy doublet (refer to Fig. 5.10(b)), there would be little variation in overall far-IR weight with temperature. In order to investigate the origin of the "filling" weight either side of the midenergy mode, we calculate the integral sum of the conductivity in the far-IR for all temperatures. The integral sum of the $c$-axis conductivity, in the form of the effective number of carriers $N_{\text {eff }}$ from equation 2.58, is displayed as solid circles in Fig. 5.12 as a function of temperature, up to an energy of $600 \mathrm{~cm}^{-1}$, or $75 \mathrm{meV}$. As the temperature decreases, an increase in $N_{\text {eff }}$ of $3.5 \times 10^{-5}$ is observed between the average $T>T_{c}$ value $\left(3.35 \times 10^{-4}\right)$ and that at $10 \mathrm{~K}\left(3.7 \times 10^{-4}\right)$. This constitutes an increase in the number of carriers in this region of $10 \%$. This is significant in comparison with the uncertainty in $N_{\text {eff }}$ of $2 \%$. The overall increase in the low-temperature far-IR spectral weight implies that charge has been transferred from a higher energy than the far-IR. As this is outside the measurement range nothing more can be 


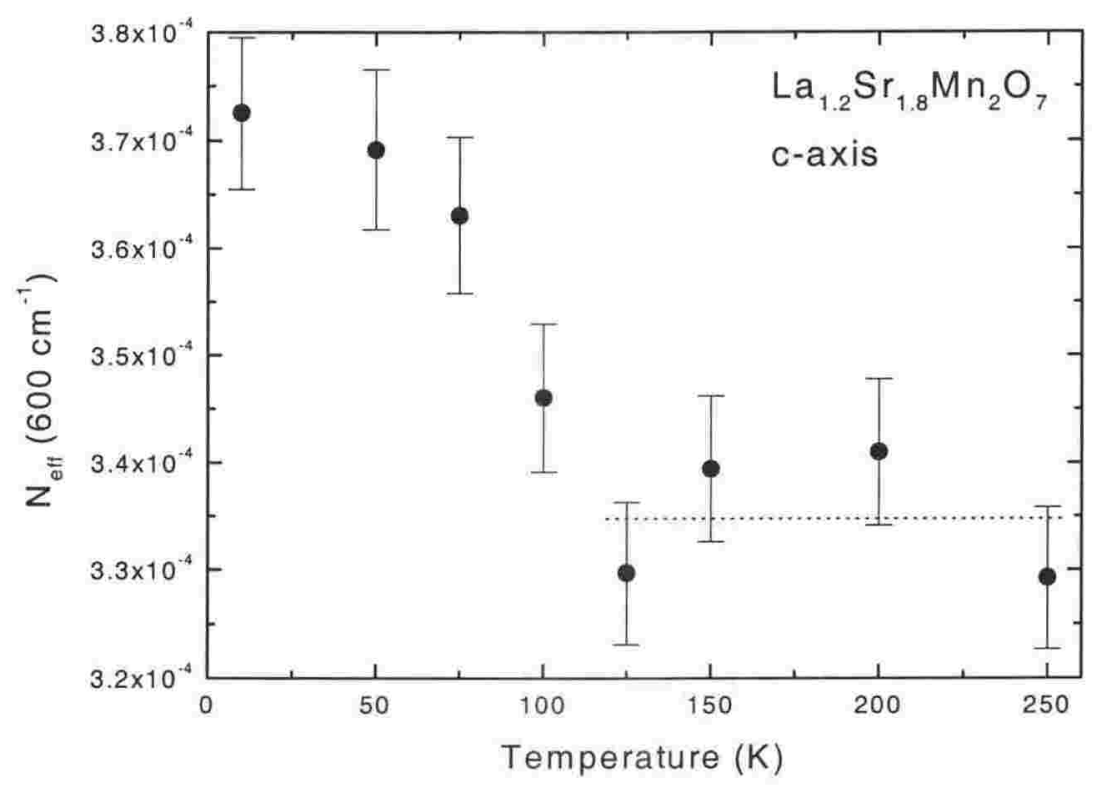

Figure 5.12: Temperature-dependence of the effective number of carriers $N_{\text {eff }}$ to $75 \mathrm{meV}\left(600 \mathrm{~cm}^{-1}\right)$ for the $c$-axis of $\mathrm{La}_{1.2} \mathrm{Sr}_{1.8} \mathrm{Mn}_{2} \mathrm{O}_{7}$. The dotted line is an indicator of possibly temperature-independent behaviour for $T>125 \mathrm{~K}$.

said except that, in comparison with the $a b$ plane, there is a small shift of spectral weight to the low energy region from higher energies.

We now turn to our investigation of the temperature-dependence of the $c$-axis "filling" weight using the definition of equation 5.1. The limits of integration were taken as $\omega_{l}=209,412 \mathrm{~cm}^{-1}$ and $\omega_{h}=279,463 \mathrm{~cm}^{-1}$. Results of this calculation are discussed and compared with the $a b$ plane calculations in the ensuing subsection.

\section{Comparison of spectral weight changes}

We finally come to a comparison of the $a b$ plane and $c$-axis spectral weights proclaiming, from the outset, that $\varsigma(T)$ displays a strikingly similar behaviour for all of the absorption features highlighted, $a b$ plane and $c$-axis alike. For display purposes, all calculations of $\varsigma$, the spectral weight change, were nor- 
malised using

$$
\bar{\varsigma}(T)=\frac{\varsigma(T)-\varsigma(200 K)}{\varsigma(10 K)-\varsigma(200 K)} .
$$

Fig. 5.13 shows the results of calculating the spectral weight temperaturedependence as described in the previous two subsections.

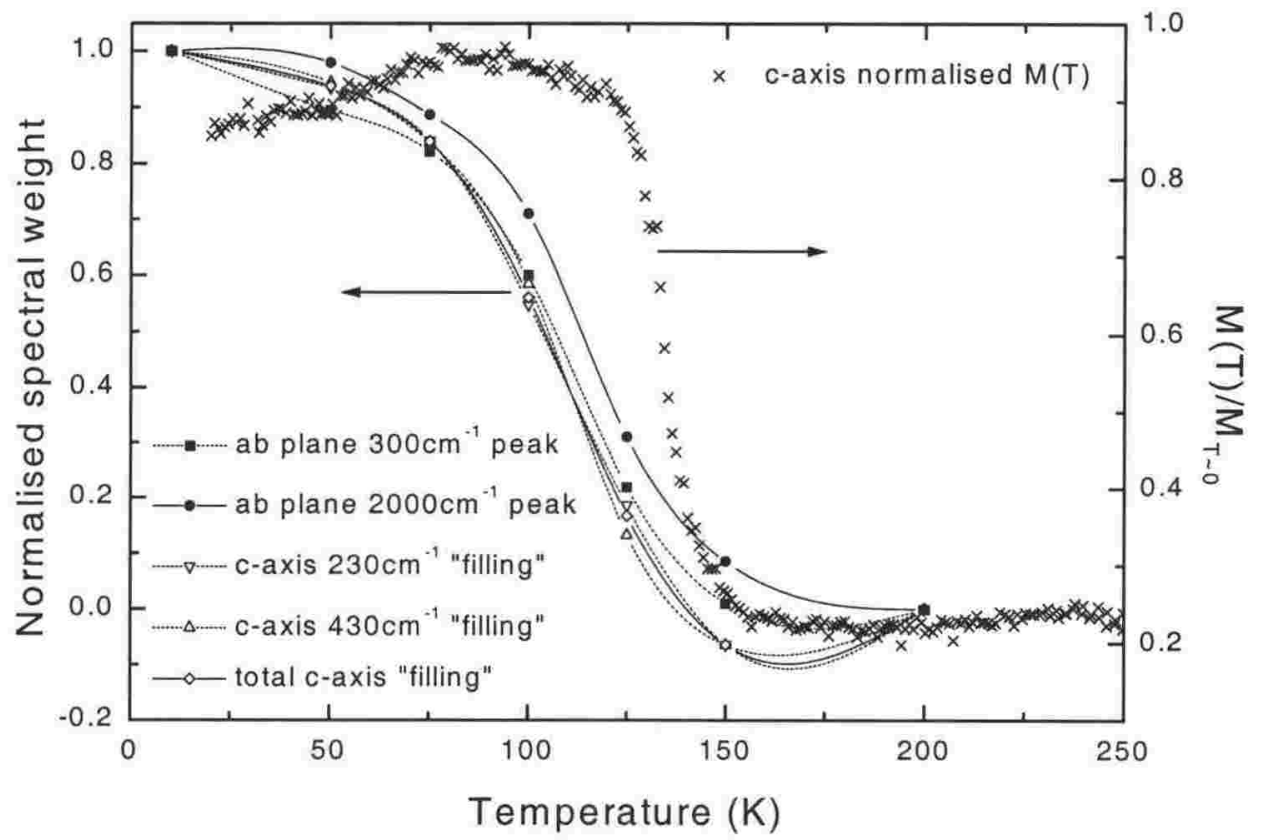

Figure 5.13: Magnetisation compared with temperature-dependence of normalised spectral weight changes, or $\bar{\zeta}(T)$, in $a b$ plane and $c$-axis $\mathrm{La}_{1.2} \mathrm{Sr}_{1.8} \mathrm{Mn}_{2} \mathrm{O}_{7}$. See text for more detail.

By following the guide-to-eye lines in Fig. 5.13 we note that:

1) $\bar{\varsigma}(T)$ for the $300 \mathrm{~cm}^{-1}$ (filled squares) and $2000 \mathrm{~cm}^{-1}$ (filled circles) peaks seen in the $a b$ plane show similar temperature-dependence; a gradual rise over a band of $\approx 150 \mathrm{~K}$ as temperature decreases. The $2000 \mathrm{~cm}^{-1}$ absorption increases at a greater rate with temperature.

2) $\bar{\varsigma}(T)$ of the $c$-axis "fillings" in the two regions of $230 \mathrm{~cm}^{-1}$ (open downpointing triangles) and $430 \mathrm{~cm}^{-1}$ (open up-pointing triangles) show almost identical temperature-dependence. 
3) $\bar{\zeta}(T)$ calculated from the sum of these two $c$-axis "fillings" (open diamonds) has similar temperature-dependence to $\bar{\zeta}(T)$ for the $a b$ plane absorption features.

The correlation between the behaviours described above is quite striking. It suggests that the driving mechanism behind each of these features is one and the same. To try and identify what that mechanism could be, we also show in Fig. 5.13 a magnetisation curve from Fig. 5.8. The $c$-axis, $H=0 \mathrm{~T}$, curve has been selected for display, but because the behaviour of $M(T)$ at $T_{c}$ appears to be independent of field and crystal orientation, this choice is fairly arbitrary.

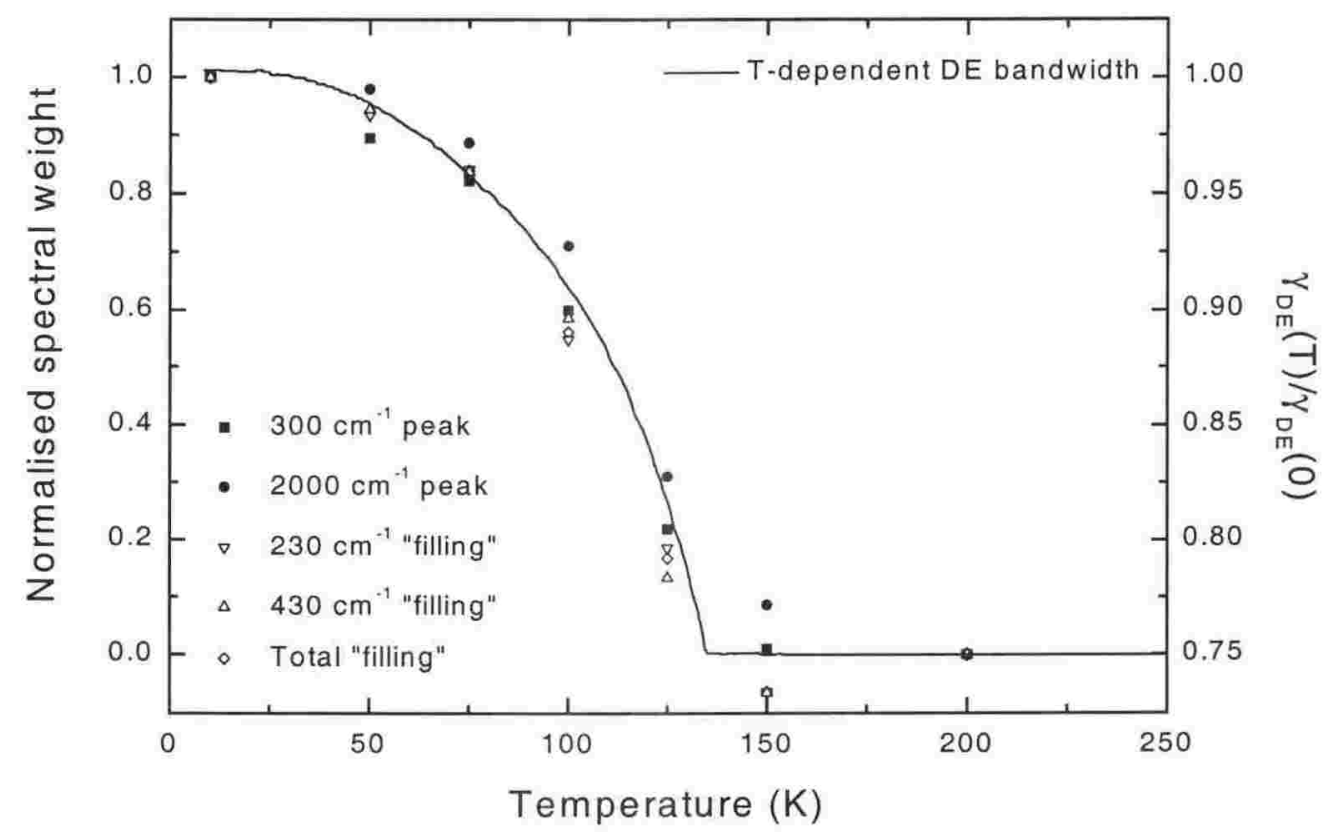

Figure 5.14: Temperature-dependent Double Exchange bandwidth (solid line) compared with temperature-dependence of spectral weight changes in $\mathrm{La}_{1.2} \mathrm{Sr}_{1.8} \mathrm{Mn}_{2} \mathrm{O}_{7}$.

Immediately obvious is that the temperature-dependence of $\bar{\zeta}(T)$ does not correspond very well to that of the magnetisation. The frequency-dependent carrier response is much more gradual than the onset of ferromagnetic order. 
It is well to point out, however, that the magnetisation measurement probes the long range magnetic order, but gives little information on short range magnetic correlations. On the other hand, $\sigma_{1}($ or $\bar{\zeta}(T))$ is more susceptible to local interactions. Thus the observation that $M(T)$ and $\bar{\varsigma}(T)$ are not well correlated does not necessarily imply that the absorption increase is not associated with magnetic order. Indeed the fact that the onsets occur at the same temperature suggests a similar underlying physics.

Turning to Fig. 5.14 however, we observe that the shape of the temperature-dependence of $\bar{\zeta}(T)$ corresponds quite well to the temperature-dependent Double Exchange bandwidth, which was discussed in section 5.1.2. This implies that a polaron could be important in describing the unusual features in $\mathrm{La}_{1.2} \mathrm{Sr}_{1.8} \mathrm{Mn}_{2} \mathrm{O}_{7}$. Indeed, we have already speculated on the possibility of the $2000 \mathrm{~cm}^{-1}$ feature as being polaronic. However, further discussion can only be speculative, and we therefore now alight from this train of thought.

\subsubsection{High-energy mode phonon analyses}

The IR response of the highest energy manganite phonon is special, in that, by in all likelihood probing the length of the Mn-O bonds, their temperaturedependence, differences between their lengths in the octahedra, it can be an indicator of orbital or charge ordering in the material. We have seen already that the $c$-axis mode splits at low temperature, and that the frequency of the $a b$ plane mode shows anomalous behaviour at $T_{c}$. We present analysis of these phenomena in this section.

Fig. 5.15 displays the integrated spectral weight for the highest energy $a b$ plane phonon and its variation with temperature. As the material passes through $T_{c}$ there is a factor of 4 increase in the phonon spectral weight, which occurs over a very narrow temperature range of about $25 \mathrm{~K}$. Displayed in the inset is the temperature-dependent spectral weight of the two lower energy phonons. By contrast, there is only a slight increase in spectral weight of the mid-energy mode as the temperature increases. The low-energy mode appears to be temperature-independent.

Fig. 5.16 displays the frequency of the high-energy mode in the $a b$ 


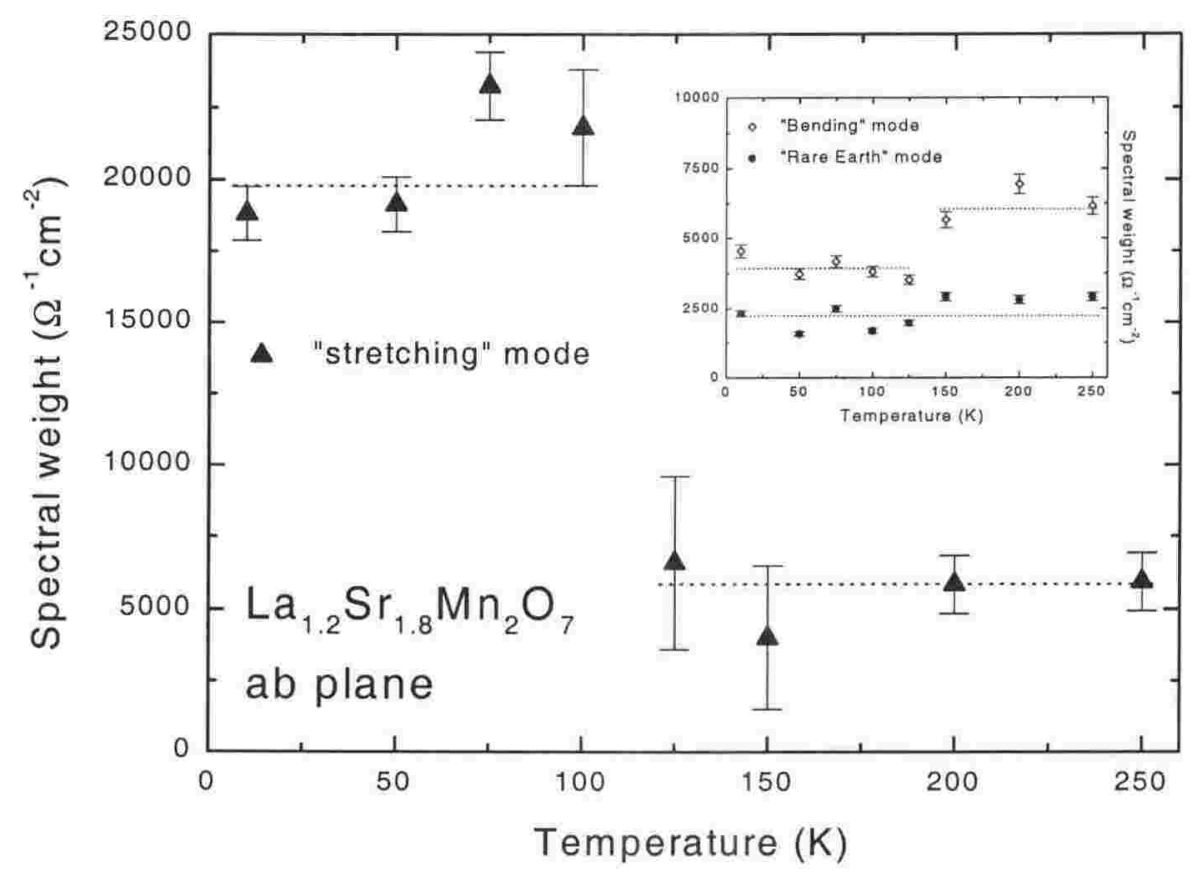

Figure 5.15: $\mathrm{La}_{1.2} \mathrm{Sr}_{1.8} \mathrm{Mn}_{2} \mathrm{O}_{7} a b$ plane phonon spectral weights. The larger uncertainty for the high-energy mode weight is due to being in the region of ellipsometry data decoupling.

plane conductivity and its variation with temperature. As discussed previously, and observed quite clearly in this diagram, this phonon stiffens significantly at $125 \mathrm{~K}$. The high-energy mode stiffening was also observed by Lee and coworkers[71]. From their $\sigma_{1}(\omega, T)$ spectra they also calculate the temperature-dependence of the centre frequency $\left(\omega_{c}\right)$ of the $1 \mathrm{eV}\left(8000 \mathrm{~cm}^{-1}\right)$ peak ascribed to a polaron absorption, and the temperature-dependence of the gap energy between the mid-IR and far-IR absorptions. Comparing these with the temperature-dependence of the centre frequency, $\omega_{c}$, of the highenergy mode, they find that the temperature-dependence of these 3 energies correlate well, each showing a peak at $T_{c}$ with softenings for $T<T_{c}$ and $T>T_{c}$. The authors concluded that such a correlation is evidence of coupling and interplay between the charge, spin and lattice degrees of freedom.

From factor group analysis we expect to see $5 c$-axis phonon modes. As- 


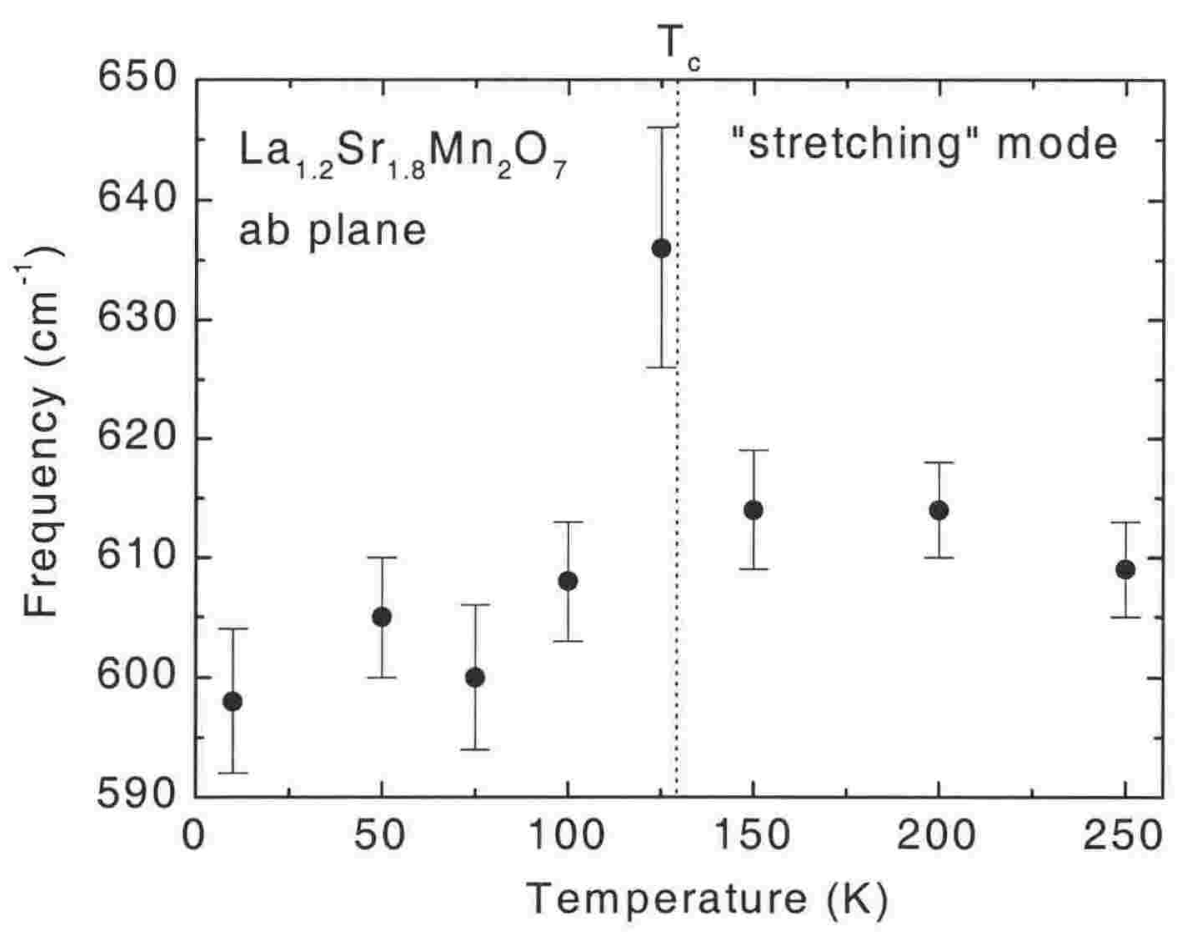

Figure 5.16: $\mathrm{La}_{1.2} \mathrm{Sr}_{1.8} \mathrm{Mn}_{2} \mathrm{O}_{7} a b$ plane high-energy mode frequency shift.

suming then that the high-energy phonon at $480 \mathrm{~cm}^{-1}$ is composed of 2 indistinguishable Lorentzian oscillators at $T>T_{c}$, and that what we termed a "filling" in a previous section at $430 \mathrm{~cm}^{-1}$ is actually the low energy side of a phonon, we fit the $480 \mathrm{~cm}^{-1}$ absorption feature with a doublet for all temperatures, and show the resultant change in frequency, width and strength in Fig. 5.17. We note, before going any further, that this analysis assumes that a local environment approximation is applicable in this instance. Due to the long range correlations in any crystal, this serves to qualify these results somewhat. However, the analysis remains of some interest.

We observe in panel (a) that the separation energy of the two phonons almost doubles, from $30 \mathrm{~cm}^{-1}$ to $57 \mathrm{~cm}^{-1}$, as the temperature decreases from $125 \mathrm{~K}$ to $10 \mathrm{~K}$. The width and strength of each of the phonons show a crossover at $100 \mathrm{~K}$ and $125 \mathrm{~K}$, respectively, with decreasing temperature. The higher energy phonon is dominant in spectral weight for $T>T_{c}$, decreasing 


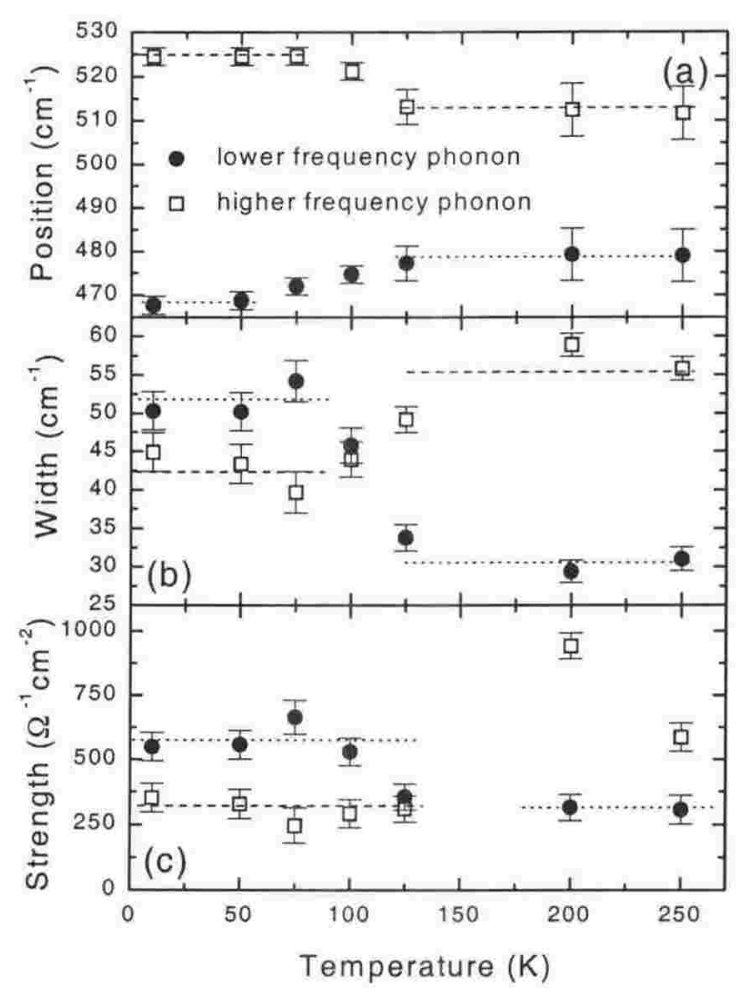

Figure 5.17: (a) centre frequency, (b) energy width and (c) strength of the high-energy mode $c$-axis phonons in $\mathrm{La}_{1.2} \mathrm{Sr}_{1.8} \mathrm{Mn}_{2} \mathrm{O}_{7}$.

in significance as the spectral weight of the lower energy phonon increases, and the material passes into the low temperature ferromagnetic phase.

An anomaly has been observed in neutron powder diffraction measurements of the unit cell dimensions of $\mathrm{La}_{1.2} \mathrm{Sr}_{1.8} \mathrm{Mn}_{2} \mathrm{O}_{7}[77,81]$, which involves an abrupt dilation of the $c$-axis lattice constant just below $T_{c}$, accompanied by an abrupt contraction of the $a$ lattice constant. Further observed is what Mitchell and coworkers[77] term a "five long, one short" geometry, that is, five of the six Mn-O bonds in the octahedron having lengths essentially the same, with the Mn-O(2) bond length (that closest to the rock salt insulating layer) being measurably longer for $T<T_{c}$. This geometry is a consequence of Jahn-Teller distortion in the layered manganite, which, by contrast with 
the perovskite manganites, is enhanced in the ferromagnetic regime. The lowering of the symmetry of the lattice lifts vibrational degeneracies and can give rise to the appearance of new phonon modes.

Given this information, we may suggest a possible explanation for the splitting of the high-energy mode. That is, the Mn-O bonds in the octahedra along the $c$-axis have split into two distinct lengths. For this change in lattice structure to give rise to a new phonon requires many conditions to be met: long-range correlation, or order, allowing the propagation of the phonon, the new phonon being optic, and the new phonon being IR active.

As it happens, at $20 \mathrm{~K}$, the $c$-axis "one long, one short" discrepancy between the Mn-O bond lengths is $2.003 \AA$ compared to $1.936 \AA$, or $3.3 \%$. This is not in itself enough to account for the $57 \mathrm{~cm}^{-1}$ splitting in the phonon. Indeed, as already mentioned, to explain a phonon doubling as a result of bond length differences assumes that a host of other conditions are also met. For further elucidation this issue must now be left to further work.

\subsection{Layered $\mathrm{Pr}_{2-2 x} \mathrm{Sr}_{1+2 x} \mathrm{Mn}_{2} \mathrm{O}_{7}$.}

The $x=0.5$ doped materials represent a special subset of the manganites which show the effects of charge order. Signatures of this behaviour have been observed most extensively in $\mathrm{LaSr}_{2} \mathrm{Mn}_{2} \mathrm{O}_{7}$; in the resistivity $[82,83,84,85]$, magnetism[82, 84, 85], transmission electron microscopy[83] and electron and $\mathrm{x}$-ray diffraction measurements[85, 86]. An optical study of one $n=2$ member, $\mathrm{LaSr}_{2} \mathrm{Mn}_{2} \mathrm{O}_{7}$, has been published[26]. The size of the lanthanide substitute ion has been observed to play a significant role in the structure and carrier dynamics of the layered materials, with the La ion, the largest, exhibiting the greatest degree of correlation of interatomic spins, thereby encouraging maximum ferromagnetic exchange interaction between Mn ions for example[87]. It has also been suggested that the compounds with larger lanthanide ions result in materials in which antiferromagnetic clusters coexist with the ferromagnetic phase[88]. In a continuing investigation of the properties of the series, the logical progression of experiments is to follow the well known lanthanide contraction across the table. Given that the ionic radius of $\operatorname{Pr}$ is 1.28 
$\AA$ compared to the La ion's $1.32 \AA$, we follow this progression by presenting an optical study of $\operatorname{Pr}_{2-2 x} \mathrm{Sr}_{1+2 x} \mathrm{Mn}_{2} \mathrm{O}_{7}(x=0.5)$.

Optical studies for members of the $n=2$ series have to date been performed only for $\mathrm{La}_{2-2 x} \mathrm{Sr}_{1+2 x} \mathrm{Mn}_{2} \mathrm{O}_{7}[26,63,71]$. To the author's knowledge this dataset represents the first attempt to determine $\sigma(\omega)$ on a non-La doped member of the $n=2$ Ruddlesden-Popper series.

\subsubsection{Magnetisation}

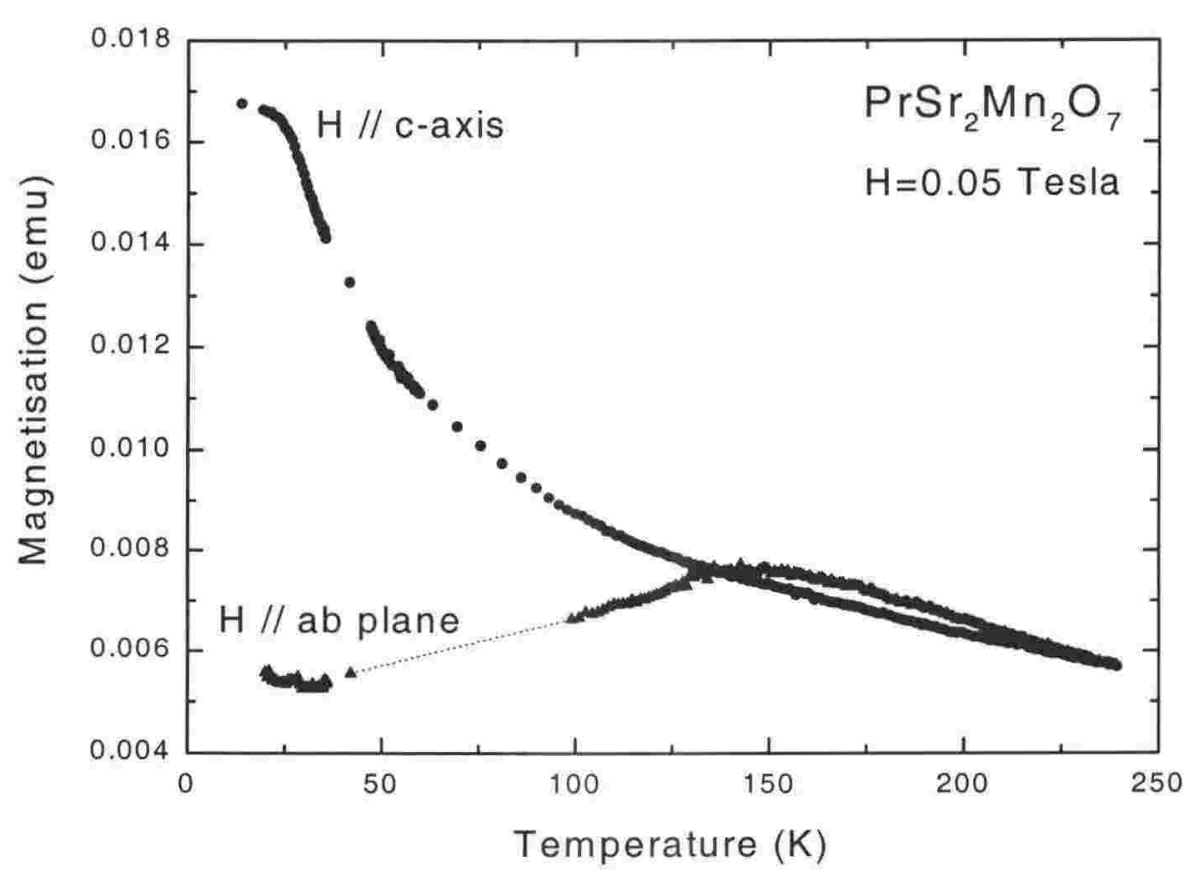

Figure 5.18: Magnetisation data with the applied field of $0.05 \mathrm{~T}$ parallel to the $c$-axis and $a b$ plane of $\operatorname{PrSr}_{2} \mathrm{Mn}_{2} \mathrm{O}_{7}$. The dotted line between $50 \mathrm{~K}$ and $100 \mathrm{~K}$ is a guide to the eye.

$M(T)$ was measured for both crystal directions of the $\mathrm{PrSr}_{2} \mathrm{Mn}_{2} \mathrm{O}_{7}$ sample that was used in the optical experiment, in exactly the same manner as described for the $\mathrm{La}_{1.2} \mathrm{Sr}_{1.8} \mathrm{Mn}_{2} \mathrm{O}_{7}$ sample. The result is displayed in Fig. 5.18. For a small applied magnetic field, $H=0.05 \mathrm{~T}$, the $a b$ plane magnetisation at 
$20 \mathrm{~K}$ is $5 \mathrm{memu}$. This increases to a maximum of $7.5 \mathrm{memu}$ at $T_{N} \approx 130 \mathrm{~K}$, plateaus to $150 \mathrm{~K}$ and then decreases again as the temperature is further increased. This behaviour signifies an antiferromagnetic transition at $130 \mathrm{~K}$, the low magnetisation at low temperature due to anti-aligned spins cancelling the overall magnetisation of the system. The $c$-axis magnetic susceptibility measurements rather display a featureless curve, reminiscent of paramagnetic behaviour. This indicates that there are no long-range correlations along the $c$-axis. Magnetisation measurements available for other large cation doped $n=2$ materials, that is, $\mathrm{LaSr}_{2} \mathrm{Mn}_{2} \mathrm{O}_{7}$ [84] and $\mathrm{NdSr}_{2} \mathrm{Mn}_{2} \mathrm{O}_{7}[84,87]$, display similar $a b$ plane behaviour.

\subsection{2 $\sigma_{1}$ by ellipsometry of $a b$ plane and $c$-axis $\mathrm{PrSr}_{2} \mathrm{Mn}_{2} \mathrm{O}_{7}$.}

Figure 5.19 shows the reduced real part of the conductivity (from hereon simply referred to as $\left.\sigma_{1}(\omega)\right)$, for $0<\omega<700 \mathrm{~cm}^{-1}$, at various temperatures measured by ellipsometry for (a) the $c$-axis, and (b) the $a b$ plane of $\mathrm{PrSr}_{2} \mathrm{Mn}_{2} \mathrm{O}_{7}$. Turning firstly to the $c$-axis $\sigma_{1}(\omega)$ we see four phonon modes centered at $\omega \approx 170 \mathrm{~cm}^{-1}, 200 \mathrm{~cm}^{-1}, 345 \mathrm{~cm}^{-1}$ and $500 \mathrm{~cm}^{-1}$ (see Table 5.2). In like manner to the $\mathrm{La}_{1.2} \mathrm{Sr}_{1.8} \mathrm{Mn}_{2} \mathrm{O}_{7}$ c-axis spectrum, the two lowest energy modes form the low-energy doublet, the $345 \mathrm{~cm}^{-1}$ feature the midenergy mode, and the $500 \mathrm{~cm}^{-1}$ peak the high-energy mode. The $345 \mathrm{~cm}^{-1}$ mode carries the greatest spectral weight of the $c$-axis modes and is quite asymmetric in shape. The $500 \mathrm{~cm}^{-1}$ mode is more Lorentzian-like, and carries less weight than the neighbouring high-energy mode. The low-energy doublet bears the lowest spectral weight of all the phonons. The reader is reminded that a comparison between the frequencies of the phonon modes for the different manganites studied, appears in table 5.2 of section 5.2.2.

In stark contrast with $\mathrm{La}_{1.2} \mathrm{Sr}_{1.8} \mathrm{Mn}_{2} \mathrm{O}_{7}$, the temperature-dependence of the $c$-axis phonon modes is minimal. A slight broadening and softening of all four of the modes is observed as the temperature is increased. This can be attributed to anharmonic effects. The highest conductivity peak value in the $c$-axis spectrum is that of the mid-energy mode, and is less than 200 


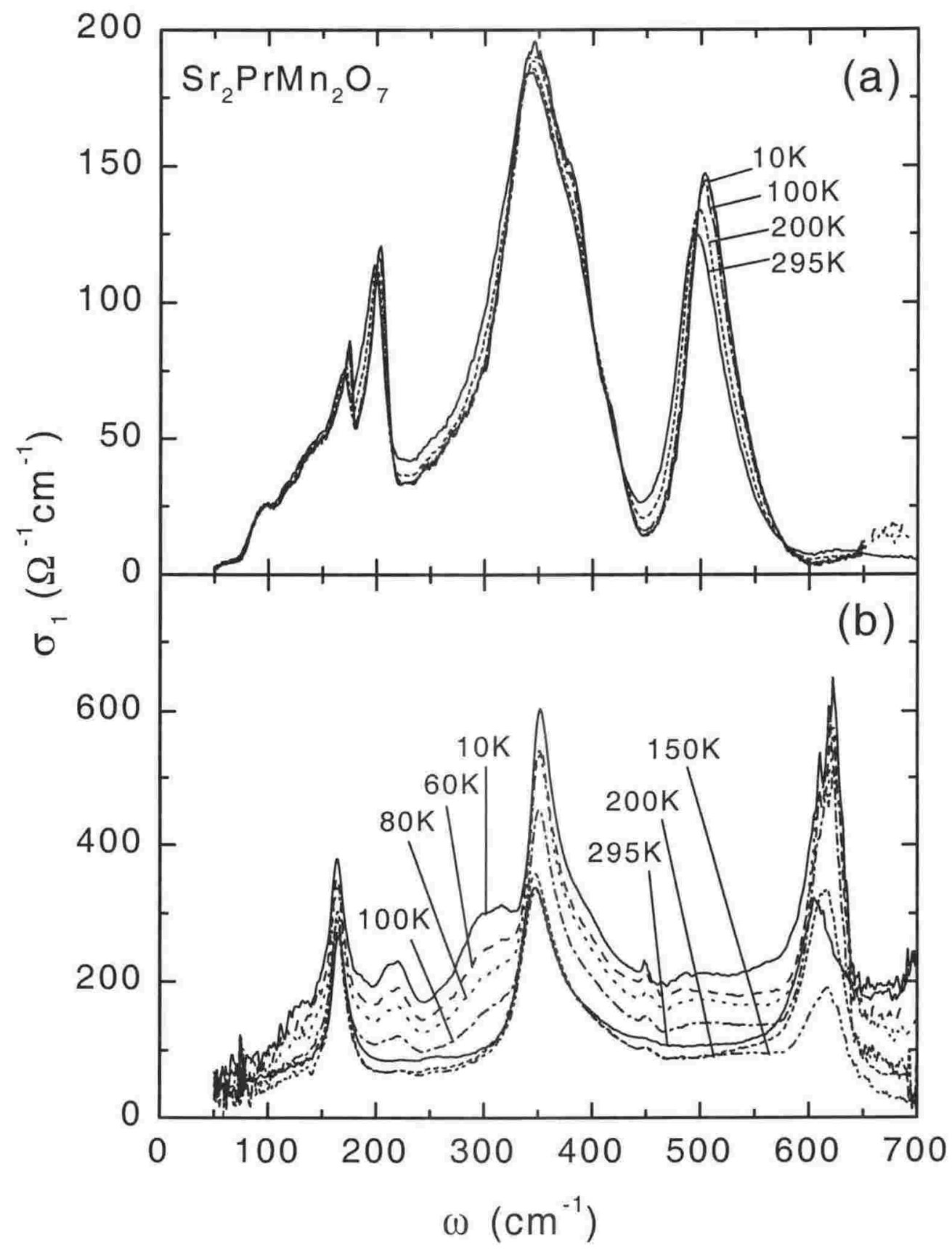

Figure 5.19: Temperature-dependent $\sigma_{1}(\omega)$ spectra for the $c$-axis (a) and $a b$ plane (b) of $\mathrm{PrSr}_{2} \mathrm{Mn}_{2} \mathrm{O}_{7}$ to $700 \mathrm{~cm}^{-1}$. 
$\Omega^{-1} \mathrm{~cm}^{-1}$. The low frequency end of the data tails off as $\omega \rightarrow 0$, to a very low conductivity also, signalling insulating behaviour along the $c$-axis.

Before discussing the striking temperature-dependence of the $a b$ plane $\sigma_{1}(\omega)$, a foundational discussion of the $a b$ plane room temperature modes is first laid. The spectra for $T>T_{N}=125 \mathrm{~K}$ will then be discussed, lastly describing the temperature-dependent behaviour that is brought about, as a result of the antiferromagnetic ordering and possible charge-ordering, for $T<T_{N}$.

At $295 \mathrm{~K}$ (the solid line in Fig. 5.19(b)) $\sigma_{1}(\omega)$ is low across the far-IR frequency region, only punctuated by three phonon modes at $\omega \approx 165 \mathrm{~cm}^{-1}$, $350 \mathrm{~cm}^{-1}$ and $620 \mathrm{~cm}^{-1}$, which are again labelled low-energy, mid-energy and high-energy modes respectively. The low-energy mode is quite sharp and symmetric is shape, in contrast especially with the mode at $350 \mathrm{~cm}^{-1}$, which is broader than the $165 \mathrm{~cm}^{-1}$ mode and asymmetric. It has a sharp slope on the low energy side, and a distinct inflection point on the high energy side which gives way to a slope of shallower gradient as $\omega$ increases. The high-energy mode displays a curious turn-up at $650 \mathrm{~cm}^{-1}$. However, the uncertainty is known to be high in this frequency region due to the reduction process (see Appendix A). Further discussion of this mode will be deferred to the presentation of the conductivity, by normal incidence reflection, in section 5.3.3.

Turning now to the $200 \mathrm{~K}$ (short dash), and $150 \mathrm{~K}$ (dash dot dot) $\sigma_{1}(\omega)$ spectra, we observe very little temperature-dependence for $T>T_{N}$ at frequencies less than $550 \mathrm{~cm}^{-1}$. The level of $\sigma_{1}(\omega)$ decreases slightly between $295 \mathrm{~K}$ and the $150 \mathrm{~K}$ and $200 \mathrm{~K}$ spectra. Though barely distinguishable in the given figure, a new mode appears in the $200 \mathrm{~K}$ and $150 \mathrm{~K}$ spectra at 220 $\mathrm{cm}^{-12}$. Fluctuations in the shape of the high-energy phonon, again, should not be interpreted due to the large uncertainty in this area.

Now turning to the $T<T_{N}$ spectra, in which the $\operatorname{PrSr}_{2} \mathrm{Mn}_{2} \mathrm{O}_{7}$ sample is in its antiferromagnetic phase, one of the most significant temperature-

\footnotetext{
${ }^{2} \mathrm{~A}$ similar feature at $450 \mathrm{~cm}^{-1}$ in these spectra we do NOT attribute to a phonon vibration. Instead, it is clearly a residual signature of a strongly interacting $c$-axis component in the $a b-c$ pseudo-dielectric function (see Appendix A). The $a b$ plane and $c$-axis reduction process removed most signs of this feature.
} 
dependent features is an increasing $\sigma_{1}(\omega)$ level, which occurs right across the far-IR energy region. The spectra at different temperatures converge in $\sigma_{1}(\omega)$ magnitude only as $\omega \rightarrow 0 \mathrm{~cm}^{-1}$. It appears that this overall rise is also contributed to by increased absorption from new IR active phonons that appear in the antiferromagnetic phase, but we will first qualitatively discuss the temperature-dependence of the phonon-independent background. Just below $T_{N}$ at $100 \mathrm{~K}$ (dash dot), the $\sigma_{1}(\omega)$ level has lifted to just above 100 $\Omega^{-1} \mathrm{~cm}^{-1}$ in the frequency region between the low-energy and high-energy phonons. The background level gradually rises as the temperature further decreases through $80 \mathrm{~K}$ and $60 \mathrm{~K}$ to $10 \mathrm{~K}$. At $10 \mathrm{~K}$ the average interphonon $\sigma_{1}(\omega)$ value is now $\approx 200 \Omega^{-1} \mathrm{~cm}^{-1}$. The gradual increase of the background was observed in the $n=2$ layered $\mathrm{LaSr}_{2} \mathrm{Mn}_{2} \mathrm{O}_{7}$ compound also[26]. We will speculate on the reason why this absorption is seen in a later section.

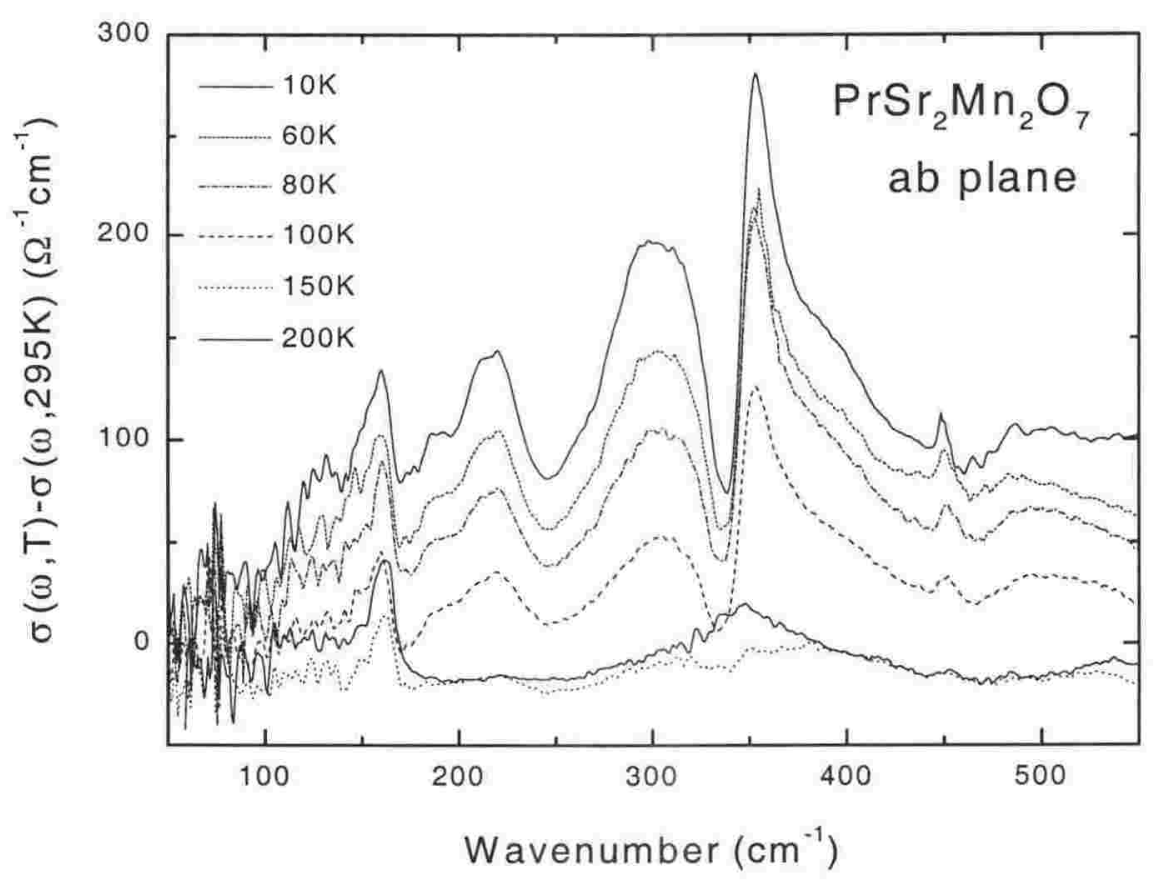

Figure 5.20: Temperature-dependent $\sigma_{1}(T, \omega)-\sigma_{1}(295 K, \omega)$ far-IR spectra of $a b$ plane $\mathrm{PrSr}_{2} \mathrm{Mn}_{2} \mathrm{O}_{7}$ by ellipsometry. 
We have mentioned that the rising conductivity background also involves contributions from both a growth in the existing phonon strengths, and the appearance of new IR active phonons. We will initially focus on the new modes and discuss each in order of increasing energy. To do this more effectively, we display the $\sigma_{1}(T, \omega)-\sigma_{1}(295 \mathrm{~K}, \omega)$ spectra between 50 and $550 \mathrm{~cm}^{-1}$ in Fig. 5.20. In like manner to Fig. 5.11, in discussion of the $\mathrm{La}_{1.2} \mathrm{Sr}_{1.8} \mathrm{Mn}_{2} \mathrm{O}_{7}$ sample, subtracting the room temperature component uncovers hidden features and is also an indicator of energy shifts of the phonons. The frequencies of these modes are presented a few pages on, in Table 5.3.

The first new feature becomes apparent in the $80 \mathrm{~K}$ spectrum at 125 $\mathrm{cm}^{-1}$, becoming more prominent in the $10 \mathrm{~K}$ spectrum where it peaks at 130 $\mathrm{cm}^{-1}$. The existence of this broad and very weak feature is, in fact, more convincingly displayed in Fig. 5.19. Returning to Fig. 5.20, a small mode appears at $180 \mathrm{~cm}^{-1}$ in the $100 \mathrm{~K}$ spectrum, growing gradually in strength with decreasing temperature. More spectacular is the increase of spectral weight, with decreasing temperature, of the $220 \mathrm{~cm}^{-1}$ mode that we argued first becomes apparent at $200 \mathrm{~K}$. As with all of the features discussed so far, the increase in the weight is gradual. The $220 \mathrm{~cm}^{-1}$ mode has a slightly asymmetric shape at all temperatures. Moving along, what appears as a shoulder on the low energy side of the $350 \mathrm{~cm}^{-1}$, mid-energy mode, at $100 \mathrm{~K}$, is transformed by the $\sigma_{1}(T, \omega)-\sigma_{1}(295 \mathrm{~K}, \omega)$ display into an asymmetric broad peak centred at $310 \mathrm{~cm}^{-1}$. Unsurprisingly, this feature grows gradually as the temperature decreases. Lastly, a shoulder on the high energy side of the mid-energy mode becomes more prominent in the Fig. 5.20 display. Centred at $400 \mathrm{~cm}^{-1}$, this feature displays the same gradual increase of spectral weight that characterises all of the phonons in $\mathrm{PrSr}_{2} \mathrm{Mn}_{2} \mathrm{O}_{7}$.

In other ways, that is, in terms of frequency shifts, the low-energy and mid-energy modes show a unique temperature dependence. A growth in the weight of the $160 \mathrm{~cm}^{-1}$ mode between room temperature and $200 \mathrm{~K}$ is evidenced by a residual peak in the $\sigma_{1}(200 \mathrm{~K}, \omega)-\sigma_{1}(295 \mathrm{~K}, \omega)$ spectrum. The asymmetry in this peak implies a frequency shift of the low-energy mode. The strength of this peak changes very little as the temperature is decreased, although its asymmetry increases, belying the slight net energy shift brought 


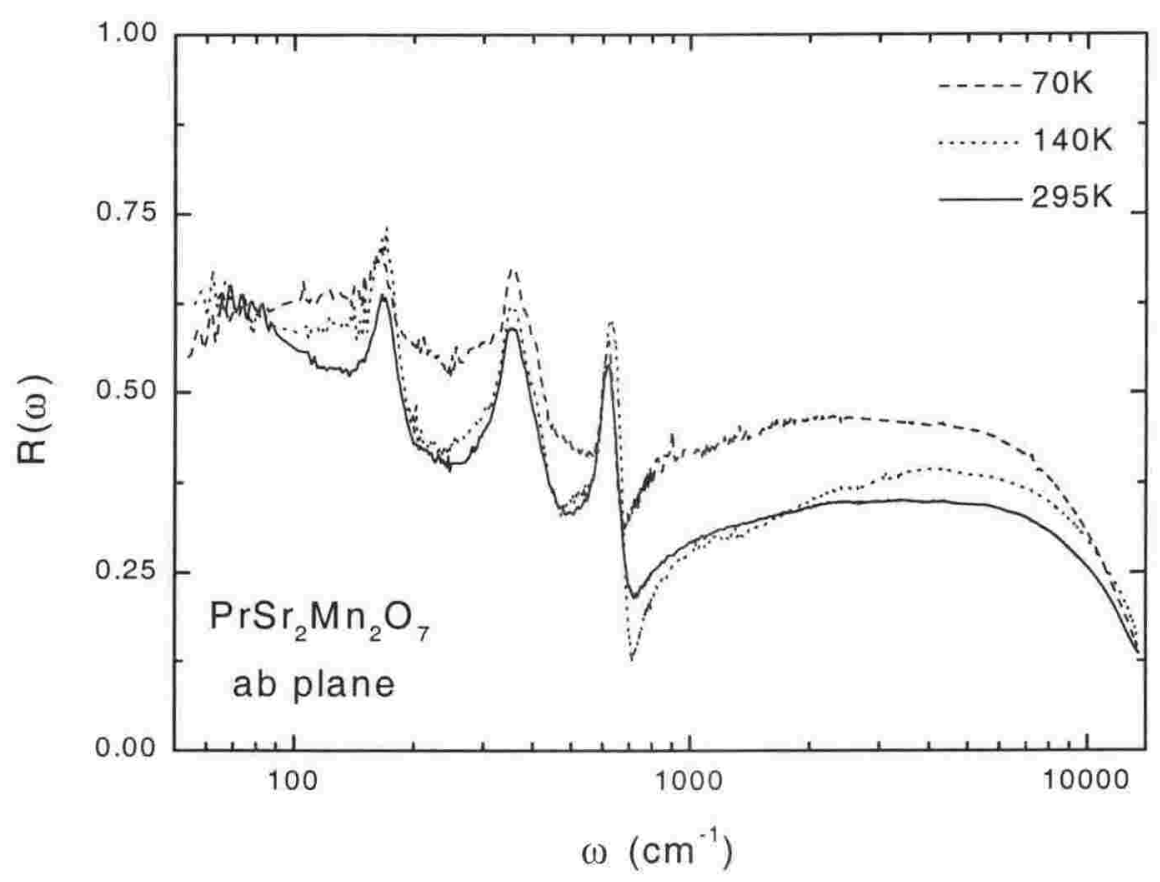

Figure 5.21: Temperature-dependent $R(\omega)$ spectra for $\operatorname{PrSr}_{2} \mathrm{Mn}_{2} \mathrm{O}_{7}$ to 13500 $\mathrm{cm}^{-1}$.

about by anharmonic effects. The mid-energy mode displays an increase in energy with decreasing temperature, as evidenced by the dramatic asymmetry in $\sigma_{1}(T, \omega)-\sigma_{1}(295 \mathrm{~K}, \omega)$, but also just as obvious upon inspection of Fig. 5.19. The onset of this shift occurs abruptly between $150 \mathrm{~K}$ and $100 \mathrm{~K}$ with a magnitude of $3 \mathrm{~cm}^{-1}$. Although the total shift in the temperature decrease from $295 \mathrm{~K}$ to $10 \mathrm{~K}$ is only $5 \mathrm{~cm}^{-1}$, about that expected due to anharmonic effects, the abruptness of the stiffening around $T_{N}$ is rather curious. There appears to be an increase in weight and a narrowing of the mode also.

\subsection{3 $\tilde{\sigma}_{\kappa \kappa}$ of $a b$ plane $\operatorname{PrSr}_{2} \mathrm{Mn}_{2} \mathrm{O}_{7}$ by normal incidence reflection}

Figure 5.21 shows the normal incidence reflectance measured from the ab plane of the $\operatorname{PrSr}_{2} \mathrm{Mn}_{2} \mathrm{O}_{7}$ sample, at three temperatures which straddle $T_{c}$. 


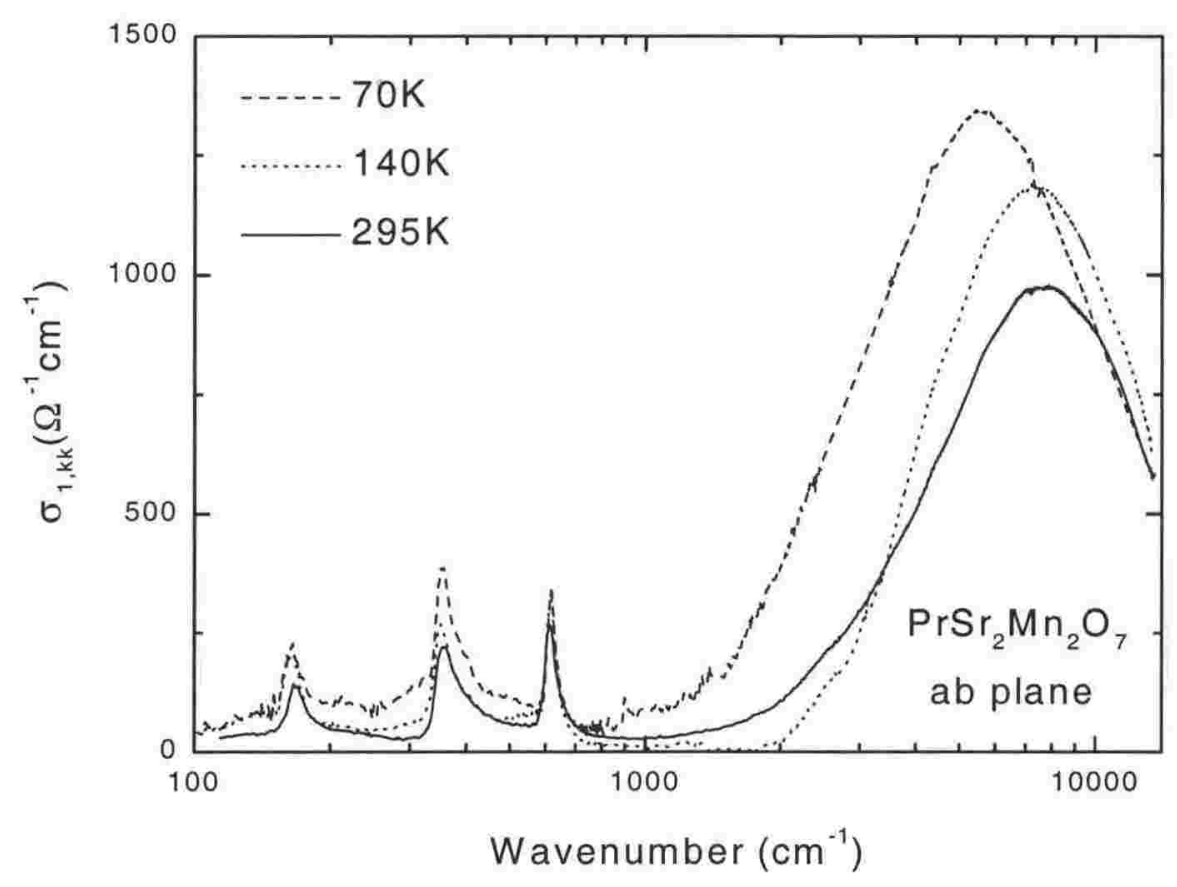

Figure 5.22: Temperature-dependent $\sigma_{1, \kappa \kappa}(\omega)$ spectra for $\operatorname{PrSr}_{2} \mathrm{Mn}_{2} \mathrm{O}_{7}$ to 13 $500 \mathrm{~cm}^{-1}$.

This measurement was performed over a greater frequency range than the ellipsometry technique and pictured is data up to $13500 \mathrm{~cm}^{-1}$. At $295 \mathrm{~K}$, the far-IR band is separated from the mid-IR region by a sharp dip in $R(\omega)$ at around $700 \mathrm{~cm}^{-1}$, which then climbs up to its peak at about $3000 \mathrm{~cm}^{-1}$, before tailing off as $\omega$ increases. At $140 \mathrm{~K}$, the dip at the division between phonon excitation and mid-IR band is more pronounced. The mid-IR band peaks at a higher energy of about $4000 \mathrm{~cm}^{-1}$. The magnitude of $R(\omega)$ is greater than that of the $295 \mathrm{~K}$ spectra for frequencies $>$ about $2000 \mathrm{~cm}^{-1}$. At $70 \mathrm{~K}$, the level of $R(\omega)$ increases across the whole frequency range, only dipping below the level of the $140 \mathrm{~K}$ spectrum at $11000 \mathrm{~cm}^{-1}$, the extreme end of the mid-IR energy band.

The phonon spectrum has been discussed amply already from the ellipsometry result, and the normal incidence reflection measurement reproduces those spectra for the most part, although the measured level of the far-IR 
reflectivity is lower (by about $R=0.1$ ) than that predicted by ellipsometric optical constants (this is attributed to loss of light by scattering, discussed further in Appendix C). The Kramers-Kronig transforms of these reflectances are shown in Fig. 5.22. In order to extract the optical constants for the sample, the reflectance was supplemented to zero frequency by a constant extrapolation. The high energy region between $13500 \mathrm{~cm}^{-1}$ and 235000 $\mathrm{cm}^{-1}$ was filled with Jung and coworkers [23] data for the $\mathrm{La}_{0.7} \mathrm{Ca}_{0.3} \mathrm{MnO}_{3}$ polycrystalline sample, used previously (see section 4.2.1). Between 235000 $\mathrm{cm}^{-1}$ and $300000 \mathrm{~cm}^{-1}$ an $\omega^{-2}$ dependence was used, and $\omega^{-4}$ decay beyond that.

We noted previously that the normal incidence reflection reproduces the far-IR phonon features of this sample for the most part. The appearance of the three phonons at room temperature, the increase in interphonon absorption with decreasing temperature, and the appearance of new phonons are all features which are reproduced. However, the reason why a discussion of the highest energy mode was left until now is because of the outstanding measurement differences in this phonon. By contrast with the fluctuation in shape and change of spectral weight beneath the mode as measured by ellipsometry, with normal incidence reflection the mode shows little temperature-dependence. If anything, it appears that the spectral weight of the high-energy phonon decreases with decreasing temperature, in stark contrast with the ellipsometry measurement.

We now focus our attention on the mid-IR region. At room temperature the mid-IR peak is centred at about $7500 \mathrm{~cm}^{-1}$, or $\omega \approx 0.9 \mathrm{eV}$. As the temperature is decreased to $140 \mathrm{~K}$, with the sample about to enter its antiferromagnetic state, this peak appears to narrow on the low energy side at least, and grows from a peak height of $1000 \Omega^{-1} \mathrm{~cm}^{-1}$ at $295 \mathrm{~K}$, to 1200 $\Omega^{-1} \mathrm{~cm}^{-1}$ for the $140 \mathrm{~K}$ spectrum. The width of the gap between the far-IR and mid-IR regions increases, so that the onset of the mid-IR peak moves from about $1500 \mathrm{~cm}^{-1}$ at room temperature, to $2000 \mathrm{~cm}^{-1}$ at $140 \mathrm{~K}$. At $75 \mathrm{~K}$, well into the antiferromagnetic phase, the mid-IR peak has shifted to 5500 $\mathrm{cm}^{-1}$ or $0.75 \mathrm{eV}$. The peak onset frequency has lowered to about $1200 \mathrm{~cm}^{-1}$. The spectral weight of the broad mid-IR peak is increasing with decreas- 
ing temperature, being presumably transferred from a higher energy than is measured in this experiment.

Having described the results, we turn to a discussion. The features which will be focused on are as follows:

1) The temperature-dependence of the mid-IR band just described and how a polaron model can be used to explain this.

2) Possible explanation of the new modes in the far-IR spectrum.

3) The increasing absorptive far-IR background in the $a b$ plane.

\subsubsection{Mid-IR band understood as a polaron}

An electron in an ionic crystal, and the associated pattern of lattice distortion that can lead to its entrapment, is the quasiparticle known as a polaron. In the manganites of this study, the crystal environment of the Mn $e_{g}$ electron is understood to undergo a Jahn-Teller distortion $[89,90]$. This removes the degeneracy of the $e_{g}$ orbital and traps the electron on the lower energy site, creating the Jahn Teller polaron. It has also been noted that, in some materials[80,91], electron-phonon coupling has significant effects on the absorption of the phonon, giving rise to asymmetric Fano line shapes. Certainly the temperature-dependent changes in phonon mode behaviours, observed here in $\mathrm{La}_{1.2} \mathrm{Sr}_{1.8} \mathrm{Mn}_{2} \mathrm{O}_{7}$ and $\mathrm{PrSr}_{2} \mathrm{Mn}_{2} \mathrm{O}_{7}$, are strongly suggestive of non-conventional dynamics.

The optical conductivity behaviours of polarons are modelled with different approaches by two authors[92, 93, 94]. Our approach is to examine the differences between a large and a small polaron, contrasting the modelled polaron conductivity behaviours with the observed spectral features in $\mathrm{PrSr}_{2} \mathrm{Mn}_{2} \mathrm{O}_{7}$.

A small polaron is an electron trapped in the coulomb field of one lattice site. A large polaron is an electron that is localised on a number of sites. Emin[92] considers the distinction between each of a small and large polaron using separate Hamiltonians. In the first case, the Hamiltonian describes the transition of the small polaron to a localised state on an adjacent site. In the large polaron Hamiltonian, the energy promotes the polaron to a free carrier 
state.

The temperature-dependence of a small polaron has also been reported. Emin[92] finds that, as the temperature decreases, the DC conductivity decreases, and the low-frequency free-carrier-like $\int \sigma_{1}(\omega) d \omega$ decreases. In other words, there is a decrease in low-energy spectral weight, which is transferred to a band in the mid-IR. This is in contrast to that which is observed in $\mathrm{PrSr}_{2} \mathrm{Mn}_{2} \mathrm{O}_{7}$, for which the spectral weight transfer is to lower frequencies with decreasing temperature.

Millis and coworkers[93, 94] study the effects on the conductivity of materials with a continuum of carrier freedom; from fermi liquid to trapped electron behaviour. Variation between the extremes is achieved by varying the degree of coupling of electron to phonons, using a parameter $\lambda$; the ratio of the lattice binding energy to the kinetic energy of the electron selftrapped in the lattice. The distinctions between a large and a small polaron are achieved by varying $\lambda$. They apply their polaron model specifically to the CMR manganite conductivity.

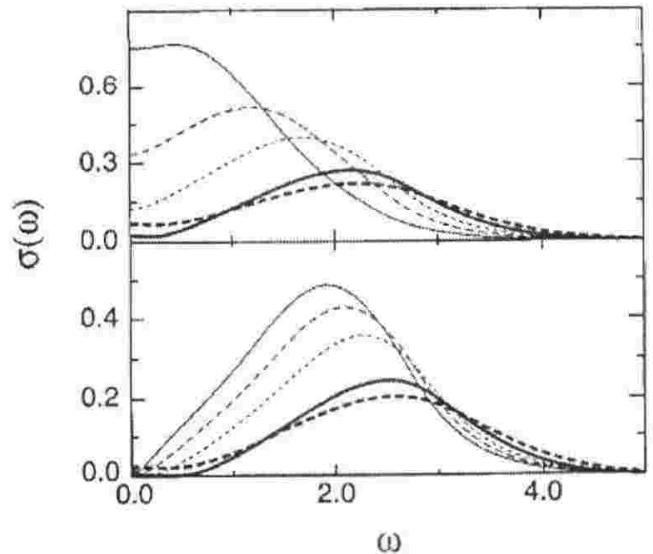

Figure 5.23: Calculated polaron absorption behaviour of the conductivity[94]. Upper and lower panels correspond to $\lambda=1.08$ and 1.15 respectively. $T=0.02$ (light solid line), $T=T_{c} / 2$ (light dashed line), $T=3 T_{c} / 4$ (light dotted line), $T=T_{c}$ (heavy solid line) and $T=2 T_{c}$ (heavy dashed line). Note that " $\sigma$ " refers to $\sigma_{1}$, and that the frequency $\omega$ is normalised to the polaron binding energy.

Displayed in Fig. 5.23 is the modelled temperature-dependence of $\sigma_{1}(\omega)$ 
spectra with different $\lambda$ from reference [94]. The top panel has $\lambda=1.08$, corresponding to a scenario with an electron that is fairly well localised at room temperature, but which is freed up as temperature decreases, as observed in the DC conductivity increase. The bottom panel has $\lambda=1.15$, representing a small polaron, or a very well localised electron.

The temperature evolution of the mid-IR conductivity band in $\mathrm{PrSr}_{2} \mathrm{Mn}_{2} \mathrm{O}_{7}$ shows qualitative agreement with the lower panel of Fig. 5.23. With respect to $T_{N}$, the temperatures presented in Fig. 5.22 are $70 \mathrm{~K}=0.54 T_{N} \approx T_{c} / 2$ (light dashed line in Fig 5.23), $140 \mathrm{~K}=1.1 T_{N} \approx T_{c}$ (heavy solid line in Fig 5.23) and $295 \mathrm{~K}=2.3 T_{N} \approx 2 T_{c}$ (heavy dashed line in Fig. 5.23). The shapes of the experimental data show striking similarities to the theoretical spectra at corresponding temperatures. Both bands show a shift to lower energies as the temperature is decreased. Both the bands grow in spectral weight with decreasing temperature. In both cases the spectra at $T \approx T_{c}$ also narrow and grow in peak height from their $T \approx 2 T_{c}$ widths and heights. The striking correlation implies that a polaron description as presented by Millis and coworkers would be a suitable model for the data. In that scenario, the $\operatorname{PrSr}_{2} \mathrm{Mn}_{2} \mathrm{O}_{7}$ mid-IR band can be interpreted as a polaron with a large electron-lattice effective coupling strength. The binding energy from small polaron theory[92, 95], can then be estimated as being $0.4 \mathrm{eV}\left(3200 \mathrm{~cm}^{-1}\right)$, or about half the peak energy. However, the contrast between the optical conductivity results, for the two small polaron models described, makes us fairly circumspect about pitching in either camp, despite the qualitative agreement of the $\operatorname{PrSr}_{2} \mathrm{Mn}_{2} \mathrm{O}_{7}$ data with Millis' model.

\subsubsection{Phonon analysis}

We turn our focus to the low energy phonon region of the spectrum. As discussed in section 2.4.3, factor group analysis for this crystal structure (in the non-charge-ordered, paramagnetic state) gives the IR active modes $5 A_{2 u}+6 E_{u}$. The $5 A_{2 u}$ modes correspond to $z$ or $c$-axis excitations, and the 6 doubly degenerate $E_{u}$ frequencies to $x, y$ or $a b$ plane phonons. Only 4 of the 5 expected $c$-axis or $A_{2 u}$ modes are clearly discernible in the measured 
far-IR $\sigma_{1}(\omega)$ of Fig. 5.19(a), however we remarked that the mid-energy mode is asymmetric, and could consist of two indistinguishable phonons. Unlike the $c$-axis optical spectra of $\mathrm{La}_{2-2 x} \mathrm{Sr}_{1+2 x} \mathrm{Mn}_{2} \mathrm{O}_{7}(x=0.4)$, for which the same irreducible representation applies, which displays four modes in the far-IR for $T>T_{c}$, and five modes for $T<T_{c}$, there is no temperature-dependence of $\sigma_{1}(\omega)$ along the $\operatorname{PrSr}_{2} \mathrm{Mn}_{2} \mathrm{O}_{7} c$-axis. The inter-bi-layer interaction is weaker in $\mathrm{PrSr}_{2} \mathrm{Mn}_{2} \mathrm{O}_{7}$ than $\mathrm{La}_{1.2} \mathrm{Sr}_{1.8} \mathrm{Mn}_{2} \mathrm{O}_{7}$ due to the smaller radius of the $\mathrm{Pr}$ dopant ion[87]. We have also observed that the magnetic signature of the $c$-axis is that of a paramagnet. The best conclusion we can arrive at, is that the absence of temperature-dependence in $\sigma_{1}(\omega)$ is consistent with a weaker $c$-axis orbital interaction, contributing to an absence of long range magnetic order.

Turning to the $a b$ plane modes, Table 5.3 tabulates the frequencies of the modes observed in the $a b$ plane of $\mathrm{PrSr}_{2} \mathrm{Mn}_{2} \mathrm{O}_{7}$ at $10 \mathrm{~K}$. The three most prominent modes apparent at all temperatures are listed in bold face type, with the assignments that have been applied to the pseudo-cubic manganites $[22]$.

\begin{tabular}{c||c|c|c|c|c|c|c|c}
$\omega\left(\mathrm{cm}^{-1}\right)$ & 130 & $\mathbf{1 6 5}$ & 180 & 220 & 310 & $\mathbf{3 5 0}$ & 400 & $\mathbf{6 1 5}$ \\
\hline \hline & & rare earth & & & & bending & & stretching
\end{tabular}

Table 5.3: Frequencies, of the observed far-IR modes for $a b$ plane $\mathrm{PrSr}_{2} \mathrm{Mn}_{2} \mathrm{O}_{7}$ at $10 \mathrm{~K}$.

As mentioned, six IR active $a b$ plane modes are expected in the irreducible representation, only three of which are observed at room temperature. When the temperature is decreased to below $T_{N}$ the mode count increases to at least eight. The overabundance of phonons below the transition temperature is consistent with the possibility of this material having a charge-ordered state at low temperature. That is, when a material is half doped, charge arranges itself so that long range alternate order of $\mathrm{Mn}^{4+}$ and $\mathrm{Mn}^{3+}$ ions occur. In this ordered scenario, the unit cell doubles in dimension and the Brillouin zone thus halves. This folds the dispersion curves back on themselves and thus doubles the number of observed $k=0$, or zone centre, phonons. However, no neutron data is available to test the idea that the new zone-centre 
modes are of similar frequency to the zone-boundary modes. Moreover, the charge-order state has only been predicted in $\mathrm{PrSr}_{2} \mathrm{Mn}_{2} \mathrm{O}_{7}$, not actually measured. In fact, the charge-order state in $\mathrm{LaSr}_{2} \mathrm{Mn}_{2} \mathrm{O}_{7}$, the only other $n=2$ layered $x=0.5$ material to have been characterised in literature, is not stable at low temperature. Given the current dearth of $\mathrm{PrSr}_{2} \mathrm{Mn}_{2} \mathrm{O}_{7}$ literature, it is insightful to investigate the La doped material and draw parallels. We do this here.

In their $\sigma_{1}(\omega)$ measurements of $\mathrm{LaSr}_{2} \mathrm{Mn}_{2} \mathrm{O}_{7}$, Ishikawa and coworkers[26] observe a strikingly similar spectrum in the far-IR to that measured here for the $\mathrm{PrSr}_{2} \mathrm{Mn}_{2} \mathrm{O}_{7}$ sample. New modes appear as the temperature is decreased through the material's $T_{N}=170 \mathrm{~K}$, the most prominent appearing at $225 \mathrm{~cm}^{-1}$ and $305 \mathrm{~cm}^{-1}$, very close to the the energies of the most prominent new phonons in $\mathrm{PrSr}_{2} \mathrm{Mn}_{2} \mathrm{O}_{7}$ (refer to Table 5.3). The low-energy, mid-energy and high-energy modes grow in strength and display increases in asymmetry, similar to $\operatorname{PrSr}_{2} \mathrm{Mn}_{2} \mathrm{O}_{7}$. The background conductivity increases with the temperature drop from $290 \mathrm{~K}$ to $10 \mathrm{~K}$, the magnitude of this change in $\sigma_{1}(\omega)$ is also of the order of that measured for $\operatorname{PrSr}_{2} \mathrm{Mn}_{2} \mathrm{O}_{7}$, or about $10^{2} \Omega^{-1} \mathrm{~cm}^{-1}$.

Three groups have published findings that the charge ordered state, which appears at $210 \mathrm{~K}$ in $\mathrm{LaSr}_{2} \mathrm{Mn}_{2} \mathrm{O}_{7}$, disappears as the temperature is further decreased through $50 \mathrm{~K}-100 \mathrm{~K}[85,86,96]$. Argyriou and coworkers further assert that it is the onset of antiferromagnetic order at $T_{N}=170 \mathrm{~K}$ which causes charge order to melt in $\mathrm{LaSr}_{2} \mathrm{Mn}_{2} \mathrm{O}_{7}[96]$. It is the antiferromagnetic order, in fact, which Ishikawa and coworkers ascribe as being responsible for the appearance of new modes in $\sigma_{1}(\omega)$ of $\operatorname{LaSr}_{2} \mathrm{Mn}_{2} \mathrm{O}_{7}$. The increase of spectral weight of new modes at $225 \mathrm{~cm}^{-1}$ and $305 \mathrm{~cm}^{-1}$ is gradual, coinciding with the gradual onset of antiferromagnetic order observed in $\mathrm{LaSr}_{2} \mathrm{Mn}_{2} \mathrm{O}_{7}[11]$. However, there exists some controversy in this area, as Kubota and coworkers[11] argue that the charge-order and antiferromagnetic phases coexist in $\mathrm{LaSr}_{2} \mathrm{Mn}_{2} \mathrm{O}_{7}$, and that charge-order is not completely lost even at low-temperature.

If we were to ascribe the new modes in $\operatorname{PrSr}_{2} \mathrm{Mn}_{2} \mathrm{O}_{7}$ to Brillouin zone folding, we may expect them to vanish with the disappearance of the charge ordered state at low temperatures. As the modes do not vanish at the low- 
est temperatures, we either conclude that charge order does not melt in $\mathrm{PrSr}_{2} \mathrm{Mn}_{2} \mathrm{O}_{7}$, or that charge order is not responsible for the appearance of the new modes. The amazing similarity between $\sigma_{1}(\omega)$ for the two materials strongly suggests that the same explanation for the appearance of new phonons applies to both $\mathrm{PrSr}_{2} \mathrm{Mn}_{2} \mathrm{O}_{7}$ and $\mathrm{LaSr}_{2} \mathrm{Mn}_{2} \mathrm{O}_{7}$. Although it cannot be tested at this time, the reduction of lattice symmetry due to distortions induced by the antiferromagnetic order appears the most likely explanation. Ishikawa and coworkers[26] assert that a charge density wave couples with a breathing type lattice distortion. This produces the deformation of the lattice which leads to the appearance of new modes for $T<T_{N}$.

The two types of order (charge and orbital) are inextricably linked in most manganites. The melting of the charge-order phase thus implies melting of both charge and orbital order. However, there is the possibility of either of charge or orbital order persisting into the low-temperature phase at the expense of the other. This decoupling of the orbital and charge order has been observed in $\mathrm{Nd}_{1-x} \mathrm{Sr}_{x} \mathrm{MnO}_{3}(x=0.55)$ [97].

\subsubsection{Disorder and far-IR absorption}

We now discuss the broad far-IR absorption observed in the $a b$ plane of $\mathrm{PrSr}_{2} \mathrm{Mn}_{2} \mathrm{O}_{7}$. As noted, this has a maximum interphonon absorption increase of about $10^{2} \Omega^{-1} \mathrm{~cm}^{-1}$ as the temperature is decreased. As noted in section 5.3.5, an absorption increase of this order is also observed in $\mathrm{LaSr}_{2} \mathrm{Mn}_{2} \mathrm{O}_{7}[26]$. The authors of that work offer no explanation for the phenomenon.

The increase in far-IR conductivity in the case of $\mathrm{La}_{1.2} \mathrm{Sr}_{1.8} \mathrm{Mn}_{2} \mathrm{O}_{7}$ was about $10^{3} \Omega^{-1} \mathrm{~cm}^{-1}$, and of a comparable magnitude with far-IR conductivity increases observed in other manganites[26, 69, 71]. These groups attribute the non-Drude like behaviour to localisation effects resulting from disorder.

Structural data for $\operatorname{PrSr}_{2} \mathrm{Mn}_{2} \mathrm{O}_{7}$ are not available, although, due to the dopant ion's smaller radius, the effects of orbital interaction and thus lattice distortion and charge disorder, have been anticipated to be much less than that of the La-doped series[98]. This might suggest a lower degree of disorder-induced localisation, and therefore be consistent with the smaller 
conductivity increase. Further work is a necessity in this area. 


\section{Chapter 6}

\section{Conclusions}

The exploration of the colossal magnetoresistance manganites has recently unveiled some of the most intriguing problems known to modern Solid State Physics. The influence of strongly correlated electrons cannot be ignored in these materials, and that interaction of spin, orbital and charge gives rise to physical properties which, at least, give the condensed matter scientist an appealing theoretical and experimental challenge, and at best, could underpin the technologies of the future.

Optical conductivity studies measure the overall absorption response of a material. The disentanglement of the constituent contributions to that response gives the individual electronic and vibrational excitations, allowing characterisation of the transitions taking place in the material. The difficulty we encounter, when applying this simple formula to the manganites, is in the mixing of the contributing excitations. As mentioned in this thesis, there are at least two different theoretical descriptions of electron-phonon interactions, both giving quite different ideas of what a conductivity contour might look like. The modelling of features displaying strong orbital, charge and spin correlations is thus a problem that limits our characterisation of experimental optical data. The goal of the project was to measure the conductivity, identify some of the electronic and vibrational transitions taking place in the material, and make qualitative comment on which models of electron-phonon interaction adequately describe the data.

The essence of this thesis, then, is a careful and wide ranging optical 
experiment on four different samples of manganite, representatives of many of the different physical behaviours exhibited in the manganite family. Each sample surface has been prepared carefully for reflectivity measurements, bearing in mind the surface effects on a probing electric field and compensating for these effects when necessary, by either annealing strain damaged sample surfaces or using freshly cleaved surfaces. Each sample was also characterised in terms of the temperature-dependence of its magnetic order. Selected samples were examined for crystal orientation with respect to the probing electric field and structural characteristics.

The anisotropic temperature-dependent and, in some instances, $\mathrm{H}$-dependent magnetisation of each of the four samples was carefully measured, both before and after annealing treatments in the instance of the pseudo-cubic manganites. This measurement not only allowed us to identify the transition temperatures of our materials, and the nature of magnetic order in them, but also to monitor the pseudo-cubic samples for bulk changes possibly brought about by the thermal treatments applied to heal surface polishing strains. Scanning electron microscope measurements of the surface of annealed $\mathrm{La}_{0.9} \mathrm{Ca}_{0.1} \mathrm{MnO}_{3}$ were made, revealing a surface smooth on the scale of IR radiation. Laue $\mathrm{x}$-ray diffraction measurements enabled us to locate the $\left(\begin{array}{lll}1 & 0 & 0\end{array}\right)$ faces of the pseudo-cubic single crystals and thereby measure the optical conductivity at a high point of symmetry.

The two different techniques used to measure the frequency-dependence of the conductivity, namely normal incidence reflection and oblique incidence ellipsometry, gave results of a high photometric accuracy. The use of two independent techniques to measure the optical conductivity adds a valuable new dimension to the body of optical work already available on the manganites. To our knowledge, this is the first time ellipsometry has been used to measure the conductivity of a manganite. Spectra measured on $\operatorname{PrSr}_{2} \mathrm{Mn}_{2} \mathrm{O}_{7}$, using both ellipsometry and normal incidence techniques, afforded an extremely insightful comparison between the conductivities garnered by the two techniques.

Inversions of the normal incidence reflectivity by Kramers-Kronig transform were performed with careful attention to the extrapolations used and 
the uncertainties produced in the data as a result. The baffling result of a negative Kramers-Kronig conductivity, obtained from reflectivity spectra of unannealed $\mathrm{La}_{0.735} \mathrm{Ca}_{0.265} \mathrm{MnO}_{3}$, was successfully reproduced by a model material with a multilayer dielectric constant. The layered materials measured by ellipsometry, due to their anisotropic dielectric response, demanded much care in extraction of the $a b$-plane and $c$-axis optical conductivity. The inversion of the ellipsometric constants was performed in two steps. The first step, deducing the pseudo-dielectric functions for each sample assuming an isotropic response function. The second, reducing the $c$-axis and $a b$ plane responses, from each of the pseudo-dielectric functions measured, by a careful fitting procedure in MATLAB. While this procedure successfully procures accurate dielectric functions in most regions, it was found that a great deal of uncertainty is inherent in regions of reduction for which the solution set is almost parallel. Large but quantifiable errors were thus found in some regions of the spectra.

Each of the manganite samples investigated displayed very similar insulator-like spectra at room temperature. Three prominent phonon modes, widely acknowledged in the pseudo-cubic manganites as due to "rare earth", "bending" and "stretching" mode vibrations, appear without exception in $\mathrm{La}_{0.9} \mathrm{Ca}_{0.1} \mathrm{MnO}_{3}$ and $\mathrm{La}_{0.735} \mathrm{Ca}_{0.265} \mathrm{MnO}_{3}$. The $a b$ planes of the layered materials display at least three optic phonons at room temperature. The $c$-axis of the layered materials display at least four optic phonons at room temperature. The atomic vibrations which contribute to the extra peak, observed at low frequency for the $c$-axis spectra of $\mathrm{La}_{1.2} \mathrm{Sr}_{1.8} \mathrm{Mn}_{2} \mathrm{O}_{7}$ and $\mathrm{PrSr}_{2} \mathrm{Mn}_{2} \mathrm{O}_{7}$, can only be assigned alongside lattice dynamics calculations for this structure, which are not yet available.

The $4 \mathrm{eV}$ peak $\left(\approx 30000 \mathrm{~cm}^{-1}\right)$ due to the charge transfer transition, between the $\mathrm{O}_{2 p}$ band and either the $t_{2 g}$ or $e_{g}$ bands, is apparent in the pseudo-cubic sample spectra. The observation of temperature-dependence in the $4 \mathrm{eV}\left(30000 \mathrm{~cm}^{-1}\right)$ peak of $\mathrm{La}_{0.735} \mathrm{Ca}_{0.265} \mathrm{MnO}_{3}$ tends to favour the interpretation that this feature is due to an $e_{g}$ transition. The most mysterious and disputed feature is the broad $1 \mathrm{eV}\left(8000 \mathrm{~cm}^{-1}\right)$ peak observed at room temperature in all spectra, probably too broad, in fact, to consist 
of a single excitation. Indeed, $\mathrm{La}_{0.735} \mathrm{Ca}_{0.265} \mathrm{MnO}_{3}$ spectra at $190 \mathrm{~K}$ and at $160 \mathrm{~K}$ reveal a separation of the $1 \mathrm{eV}\left(8000 \mathrm{~cm}^{-1}\right)$ band into two distinct components. To fit this feature with multiple Lorentzian oscillators is one among many approaches toward isolating the contributing electronic transitions. This, however, was not attempted here in deference to the somewhat arbitrariness of an interpretation resulting from unascribed harmonic oscillator fits. By analogy with literature results, the data appears qualitatively consistent with an electron-lattice interaction as well as a $d$ - $d$ transition.

The question of electron-lattice, or polaron, interactions making themselves apparent in the manganites is one that is yet wrestled with. In $\mathrm{La}_{0.735} \mathrm{Ca}_{0.265} \mathrm{MnO}_{3}$ spectra, we observe that at an energy of $4000 \mathrm{~cm}^{-1}(0.5$ $\mathrm{eV}$ ), there is a noticeable crossover point of the transfer of spectral weight. The temperature-dependent effective number of carriers was calculated by integrating the conductivity spectra up to this point. It was found that $N_{\text {eff }}$ scales poorly with $\left(M(T) / M_{s}\right)^{2}$, the expected Double Exchange behaviour, supporting the large amount of evidence that Double Exchange alone cannot explain the large negative magnetoresistance effect. The spectral weight change scales well, however, with the temperature-dependent Double Exchange bandwidth, a model incorporating Jahn-Teller effects in the Double Exchange model. The results show strong supporting evidence for a Jahn Teller polaron localising the carriers in the high temperature insulating phase, and its effect dropping away gradually as temperature decreases.

In the normal incidence measurement of $\operatorname{PrSr}_{2} \mathrm{Mn}_{2} \mathrm{O}_{7}$, the mid-IR band is observed to decrease in peak frequency and grow in strength as temperature decreases, in agreement with Millis' model of a fairly strongly localised, or small, polaron. Using small polaron theory, the binding energy of the electron to the lattice is calculated to be $\approx 0.4 \mathrm{eV}\left(3200 \mathrm{~cm}^{-1}\right)$.

Another issue to consider is to what extent does an electron-lattice interaction affect the carrier motion in a material. For the purposes of this study, we have defined a resistivity which decreases with temperature as being metallic. However, with such a growing number of materials displaying conductivities which slip outside long held conventions of insulating or metallic behaviour, this definition is for pure convenience. 
For $\mathrm{La}_{0.735} \mathrm{Ca}_{0.265} \mathrm{MnO}_{3}$, the most Drude-like in its optical conductivity of the two metallic-like samples measured, as the temperature is reduced the optical spectra display the expected transfer of spectral weight from higher energies into a low energy Drude-like band. We have already mentioned that the spectral weight increase scales as though a polaron were apparent in the material, in effect slowing down the onset of metallic behaviour.

We therefore have uncovered plenty of evidence at least qualitatively consistent with models of polarons. However, the polaron models themselves are, because of the complexity of the entity in question, necessarily simplifications of the actual electron-lattice interaction. Added to that, the different polaron models can give different temperature-dependences. Even the distinction between a small, large and bi-polaron appears blurred by some models.

In the $a b$ plane conductivity of $\mathrm{La}_{1.2} \mathrm{Sr}_{1.8} \mathrm{Mn}_{2} \mathrm{O}_{7}$, as the temperature decreases through $T_{c}$, the transfer of spectral weight is to a rounded far-IR band centred at $300 \mathrm{~cm}^{-1}$, and displays a non-Drude-like absorption, a feature which may best be modelled by disorder-induced localisation. The phonons remain prominent at all temperature, not washed out as is usually the case in the spectra of metallic materials. In the mid-IR, a frequency-independent band at $2000 \mathrm{~cm}^{-1}$ grows on the low energy side of the mid-IR $1.2 \mathrm{eV}$ (10000 $\mathrm{cm}^{-1}$ ) band, which also grows in strength with decreasing temperature.

Striking temperature-dependence is also observed in the far-IR $c$-axis conductivity of $\mathrm{La}_{1.2} \mathrm{Sr}_{1.8} \mathrm{Mn}_{2} \mathrm{O}_{7}$. As well as "filling" type absorptions increasing in weight as the temperature decreases, we also observe that, as the material passes into its ferromagnetic phase, the highest energy oxygen mode phonon splits into two distinct peaks.

The temperature-dependence of these different spectral weight changes in the $\mathrm{La}_{1.2} \mathrm{Sr}_{1.8} \mathrm{Mn}_{2} \mathrm{O}_{7}$ sample show surprising correlations. We considered the $300 \mathrm{~cm}^{-1}$ absorption against the $2000 \mathrm{~cm}^{-1}$ peak and the weight increase of the extraordinary "filling" absorptions observed in the far-IR response of the $c$-axis. The correlated temperature-dependence of all of these absorptions implies that the underlying physics behind them is the same. It is further observed that the increase in long-range ferromagnetic order is not directly correlated to the increase in charge. We do however observe a correlation 
between the temperature-dependent Double Exchange bandwidth, and the spectral weight increases. This correlation may imply that electron-phonon interactions are, once again, vitally important in describing the carrier dynamics of the layered manganite.

The $\mathrm{PrSr}_{2} \mathrm{Mn}_{2} \mathrm{O}_{7}$ sample, as well as being yet untouched in literature, displays curious behaviour. The appearance of new modes with decreasing temperature is accompanied by an increase of the conductivity level in the far-IR and, as mentioned, the mid-IR band displays interesting increases in weight and decreases in frequency. In the meantime, the $c$-axis far-IR response is temperature-independent. All of the new physics appears to be restricted to the $a b$ plane.

The increase in spectral weight of the phonons appears correlated with the onset of antiferromagnetic order in the plane. The appearance of new modes, despite suggesting a folding back of the Brillouin zone, will probably be shown to be uncorrelated with charge-order in $\operatorname{PrSr}_{2} \mathrm{Mn}_{2} \mathrm{O}_{7}$. However, much of the description and analysis of $\mathrm{PrSr}_{2} \mathrm{Mn}_{2} \mathrm{O}_{7}$ has been done by analogy with behaviours observed in literature $\mathrm{LaSr}_{2} \mathrm{Mn}_{2} \mathrm{O}_{7}$. While this has served as an extremely insightful parallel, it will quickly be supplanted by measurements of $\rho(T)$ and structure on the $\operatorname{PrSr}_{2} \mathrm{Mn}_{2} \mathrm{O}_{7}$ material itself.

In short, some extremely interesting new problems in the manganite field have been uncovered and significant insight gained. Definite conclusions as to what contributes to the rich conductivity spectra in the featured samples, however, must await adequate models to describe them.

\subsection{Further work}

More supplementary experiments on the very interesting $\operatorname{PrSr}_{2} \mathrm{Mn}_{2} \mathrm{O}_{7}$ sample would provide a more focused framework within which to interpret results. Measurement of the resistivity would confirm the existence of charge order in $\mathrm{PrSr}_{2} \mathrm{Mn}_{2} \mathrm{O}_{7}$ and indicate whether that state is melted by antiferromagnetism. 


\section{Appendix A}

\section{Ellipsometric constant reduction}

In this appendix, we briefly discuss the uncertainties associated with the reduction, or decoupling, of the $a b$ plane and $c$-axis spectra measured by ellipsometry. In motivating this, we display the $a b-c$ plane pseudo-dielectric function in the form of the unreduced $\sigma_{1}(\omega)$ for $\operatorname{PrSr}_{2} \mathrm{Mn}_{2} \mathrm{O}_{7}$ and $\mathrm{La}_{1.2} \mathrm{Sr}_{1.8} \mathrm{Mn}_{2} \mathrm{O}_{7}$. These spectra clearly show the striking interaction of the $c$-axis dielectric constant with that of the $a b$ plane. Because of the possible confusion inherent in the notation, we refer to $a b-c$ and $c-a b$ functions as pre- or unreduced, and to $a b$ and $c$ functions as reduced.

\section{A.1 Pre-reduction spectra}

Fig. A.1(a) displays the unreduced $\sigma_{1}(\omega, T)$ for $\operatorname{PrSr}_{2} \mathrm{Mn}_{2} \mathrm{O}_{7}$. Upon comparison with the far-IR reduced spectrum for $\mathrm{PrSr}_{2} \mathrm{Mn}_{2} \mathrm{O}_{7}$, shown in section 5.3.2, there are marked differences, as indicated by arrows in the figure. At about $460 \mathrm{~cm}^{-1}$ there is a sharp asymmetric peak present for all temperatures, which grows more pronounced as the temperature is decreased. A dramatic splitting of the "stretching" phonon peak at about $630 \mathrm{~cm}^{-1}$ is also observed. The resolution into 2 distinct peaks at the lowest temperatures is in striking contrast to the single peak in the reduced $a b$ plane spectrum. Also evident in the pseudo-dielectric function is the growth of an asymmetric peak at $\omega \approx 720 \mathrm{~cm}^{-1}$ with decreasing temperature. 

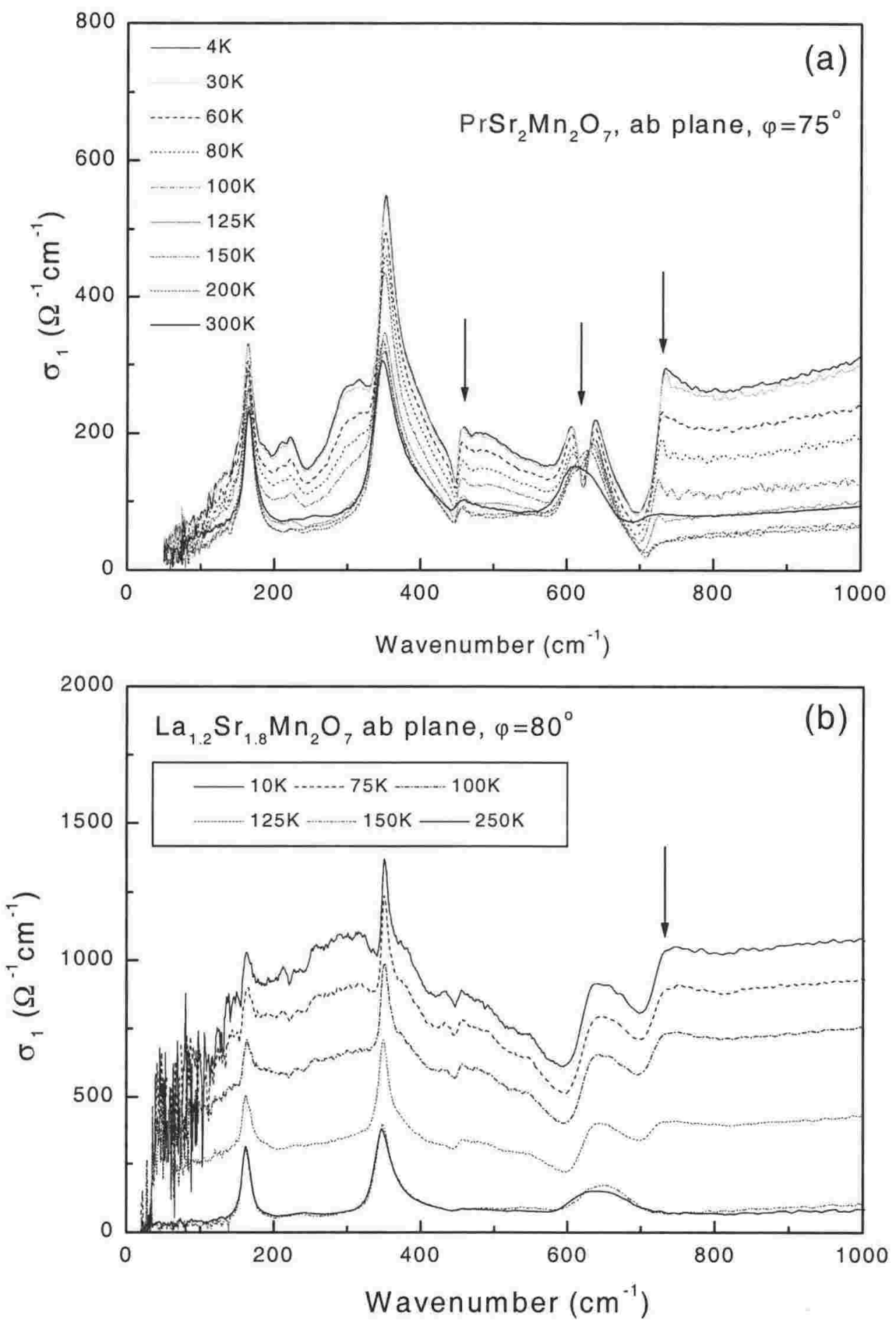

Figure A.1: Unreduced temperature-dependent far-IR $\sigma_{1}(\omega)$ spectra for $\mathrm{PrSr}_{2} \mathrm{Mn}_{2} \mathrm{O}_{7}$ (a) and $\mathrm{La}_{1.2} \mathrm{Sr}_{1.8} \mathrm{Mn}_{2} \mathrm{O}_{7}$ (b) measured by ellipsometry from the $a b$ plane. 
The unreduced spectra for $\mathrm{La}_{1.2} \mathrm{Sr}_{1.8} \mathrm{Mn}_{2} \mathrm{O}_{7}$ are shown in Fig. A.1(b). These spectra are less dramatically different from their reduced counterparts in section 5.2.2. As indicated by the arrow in Fig. A.1(b), there is an asymmetric peak at $740 \mathrm{~cm}^{-1}$ which is, again, due to a strongly interacting $c$-axis component at this energy.
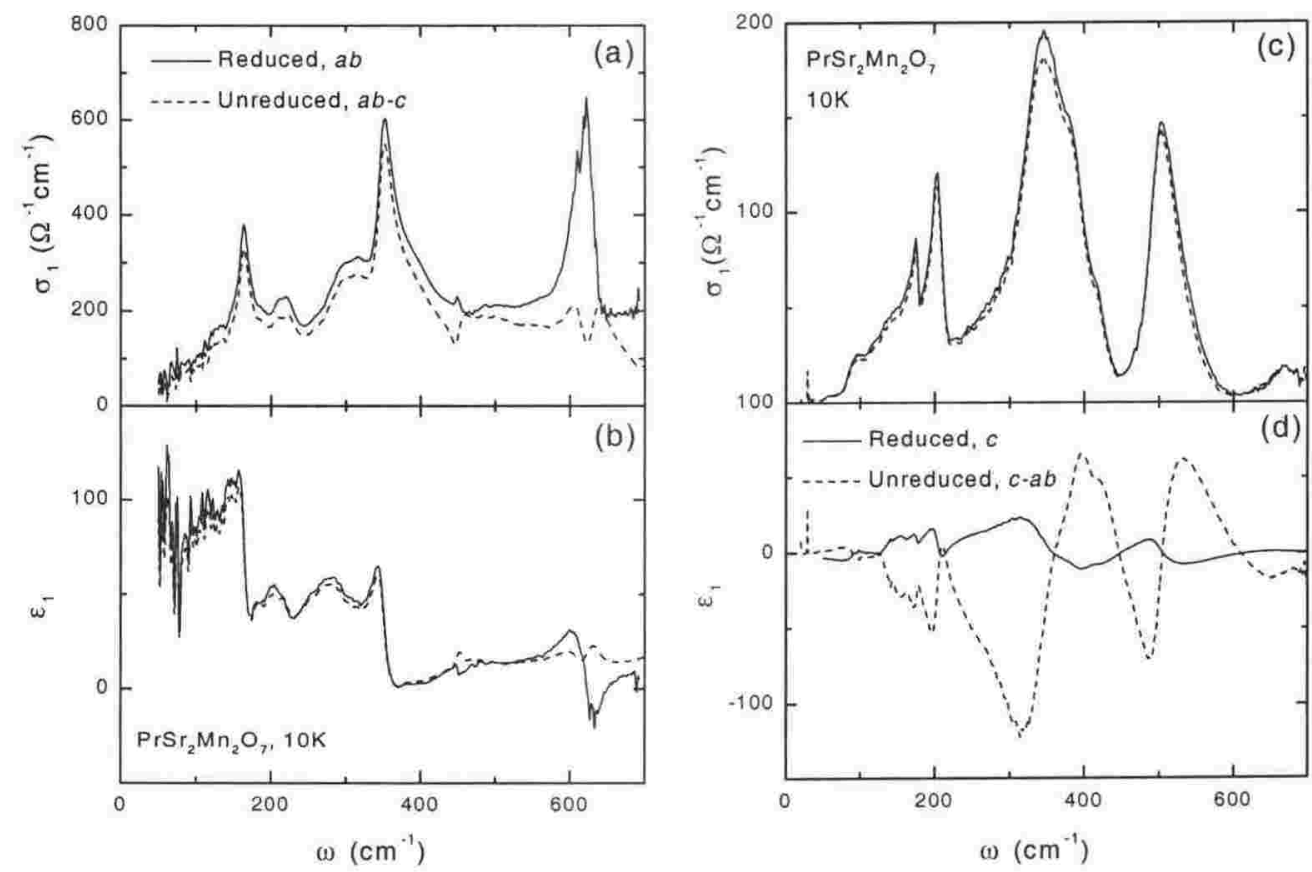

Figure A.2: Far-IR reduced (solid lines) and unreduced (dashed lines) $\sigma_{1}(\omega)$ (a), (c) and $\epsilon_{1}(\omega)(\mathrm{b}),(\mathrm{d})$ spectra for $a b$ plane (a), (b) and $c$-axis (b), (d) for $\mathrm{PrSr}_{2} \mathrm{Mn}_{2} \mathrm{O}_{7}$ at $10 \mathrm{~K}$.

As can be appreciated from Fig. A.1, the most dramatic contrasts between reduced and unreduced $\sigma_{1}(\omega)$ occur at the lowest temperatures. We thus present a more comprehensive comparison of optical constants using the spectra at $10 \mathrm{~K}$. In Fig. A.2 we show a contrast of the unreduced (dashed lines) spectra with the reduced (solid lines) spectra for $\operatorname{PrSr}_{2} \mathrm{Mn}_{2} \mathrm{O}_{7}$. In panel (a) and (c) of Fig. A.2 are plotted $\sigma_{1}(\omega)$ for the $a b$ plane and $c$-axis respectively. As already commented on, the $a b$ response is markedly different from the pseudo-dielectric function measured from the $a b$ plane. By contrast how- 
ever, the $c$-axis response is very similar to the pre-reduced response measured from the $c-a b$ plane. $\epsilon_{1}(\omega)$ measured before and after reduction are displayed in panels (b) and (d) for the $a b$ plane and the $c$-axis respectively. The reduced $a b$ plane $\epsilon_{1}(\omega)$ shows quite a deviation from the $a b-c$ response for $\omega>550$ $\mathrm{cm}^{-1}$, however the real changes are most evident in the $c$-axis reduction in panel (d). The $c$ - $a b$ spectrum displays a dramatic and large interaction of the $a b$ plane response with the $c$-axis response, which is removed upon reduction. This is in contrast to the far-IR ellipsometric constants measured for $\mathrm{La}_{2} \mathrm{CuO}_{4+\delta}[54,99]$ and $\mathrm{YBa}_{2} \mathrm{Cu}_{3} \mathrm{O}_{7}[54]$, both of which are approximately uniaxially anisotropic in structure. For these materials it has been suggested[40], and was subsequently shown, that the $c$ - $a b$ pseudo-dielectric function is an excellent approximation to the $c$-axis dielectric function $[54,99]$.
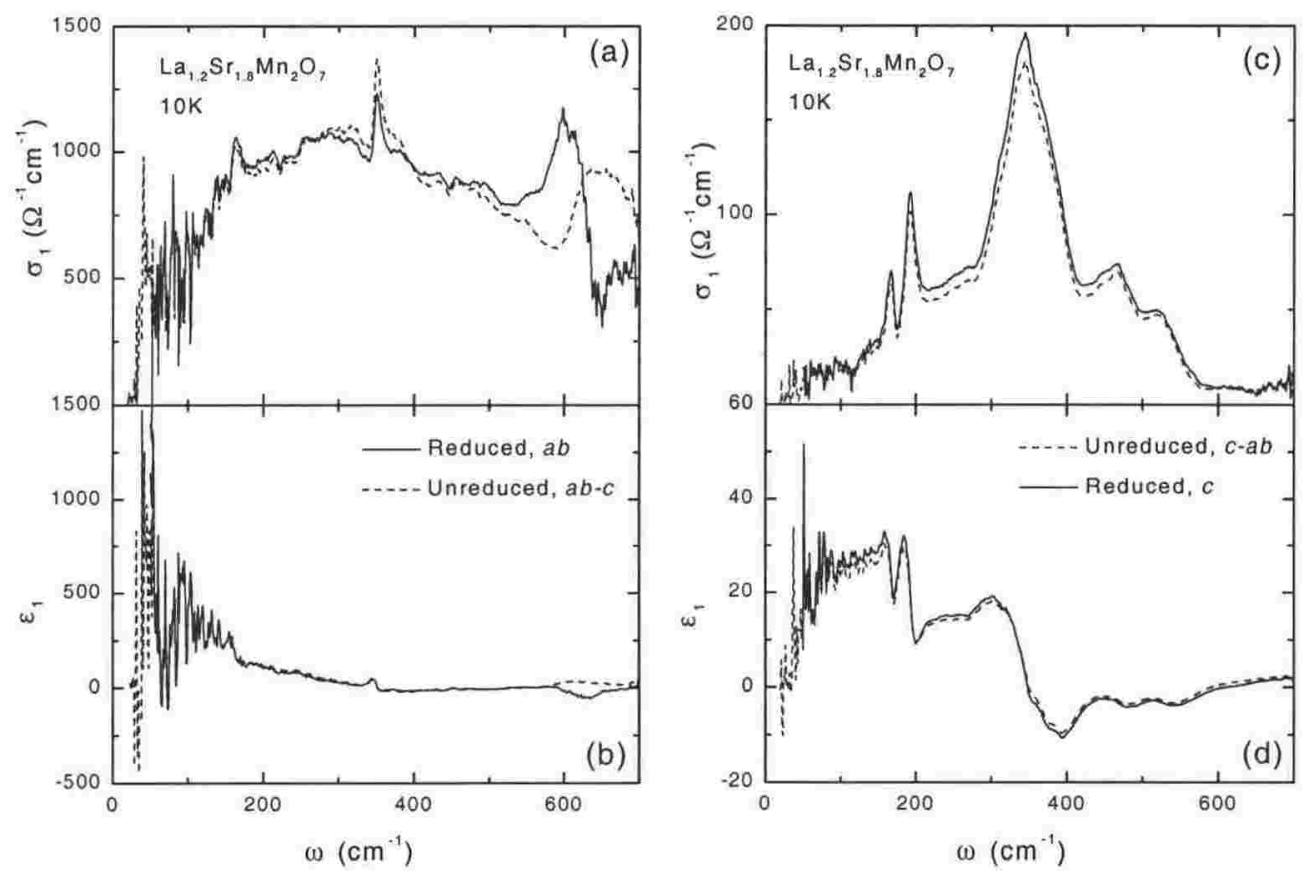

Figure A.3: Far-IR reduced (solid lines) and unreduced (dashed lines) $\sigma_{1}(\omega)$ (a), (c) and $\epsilon_{1}(\omega)$ (b), (d) spectra for $a b$ plane (a), (b) and $c$-axis (b), (d) for $\mathrm{La}_{1.2} \mathrm{Sr}_{1.8} \mathrm{Mn}_{2} \mathrm{O}_{7}$ at $10 \mathrm{~K}$.

The extent of mixing observed in $\mathrm{PrSr}_{2} \mathrm{Mn}_{2} \mathrm{O}_{7}$ is not observed in the 
$\mathrm{La}_{1.2} \mathrm{Sr}_{1.8} \mathrm{Mn}_{2} \mathrm{O}_{7}$ sample. Fig. A.3 displays a contrast of the unreduced (dashed lines) $\sigma_{1}(\omega)(\mathrm{a}),(\mathrm{c})$ and $\epsilon(\omega)$ (b), (d) spectra with the reduced (solid lines) spectra, for $a b$ plane (a), (b) and $c$-axis (b), (d). In this set, the reduction produces significant shifts in the $a b$ plane $\sigma_{1}$ and $\epsilon_{1}$ for $\omega>500$ $\mathrm{cm}^{-1}$, but changes to the $c$-axis appear frequency-independent, with just a slight shift in magnitude of $\sigma_{1}$ and $\epsilon_{1}$.

\section{A.2 Uncertainties associated with reduction}

The "black box" fitting program used to reduce the pseudo-dielectric constants gave slightly different fits in the region of the solution, depending on the start parameters used. This in turn gave us an indication of the uncertainty inherent in the reduction. As shown, for some of the spectra, the difference upon reduction is minimal. The uncertainty in these areas is due simply to that of the measurement, or $\pm 3 \%$. However, in regions where fits to the $a b$ and $c$-axis optical constants were somewhat divergent, the uncertainty increases to $\pm 5 \%$. For the highest energy regions in the Far-IR $a b$ plane spectra, that is, for $550 \mathrm{~cm}^{-1}<\omega<700 \mathrm{~cm}^{-1}$, the uncertainty was estimated to be no more than $40 \%$, but at least $10 \%$. 


\section{Appendix B}

\section{Deduction of the $c$-axis response}

This appendix contains figures pertinent to section 4.4 of Chapter 4 , which are contained here in order to streamline the text of that discussion. We are concerned with the extraction of the $c$-axis optical response from two measurements on the $a b$ plane: a normal incidence measurement, probing the exclusively $a b$ plane response, and an oblique incidence ellipsometry measurement, which, for an anisotropic sample ${ }^{1}$, also contains some element of the $c$-axis response. Full details are available in sections 4.4 and 2.2.4.

Before applying the formulae for that extraction to the $\mathrm{PrSr}_{2} \mathrm{Mn}_{2} \mathrm{O}_{7}$ data, various synthetic datasets were tested. Firstly, we consider an anisotropic material with an $a b$ plane dielectric constant consisting of a Lorentzian oscillator, centred at $350 \mathrm{~cm}^{-1}$, and a $c$-axis component made up of an identical Lorentzian oscillator centred at $500 \mathrm{~cm}^{-1}$. The dielectric response measured by ellipsometry, using a beam angle of $75^{\circ}$, is displayed in the inset of Fig. B.1(a), along with the response of the $a b$ plane. The main panel in (a) shows the expected and deduced $c$-axis responses, the good agreement between them signifying the applicability and correctness of the expressions used.

Secondly, the $a b$ plane was modelled as a metal, with a Drude function of the same strength and width as the Lorentzian previously used. As seen in Fig. B.1(b), where the routine was able to converge on a solution,

\footnotetext{
${ }^{1}$ Remembering that, in this discussion, we are only concerned with materials of uniaxial anisotropy.
} 

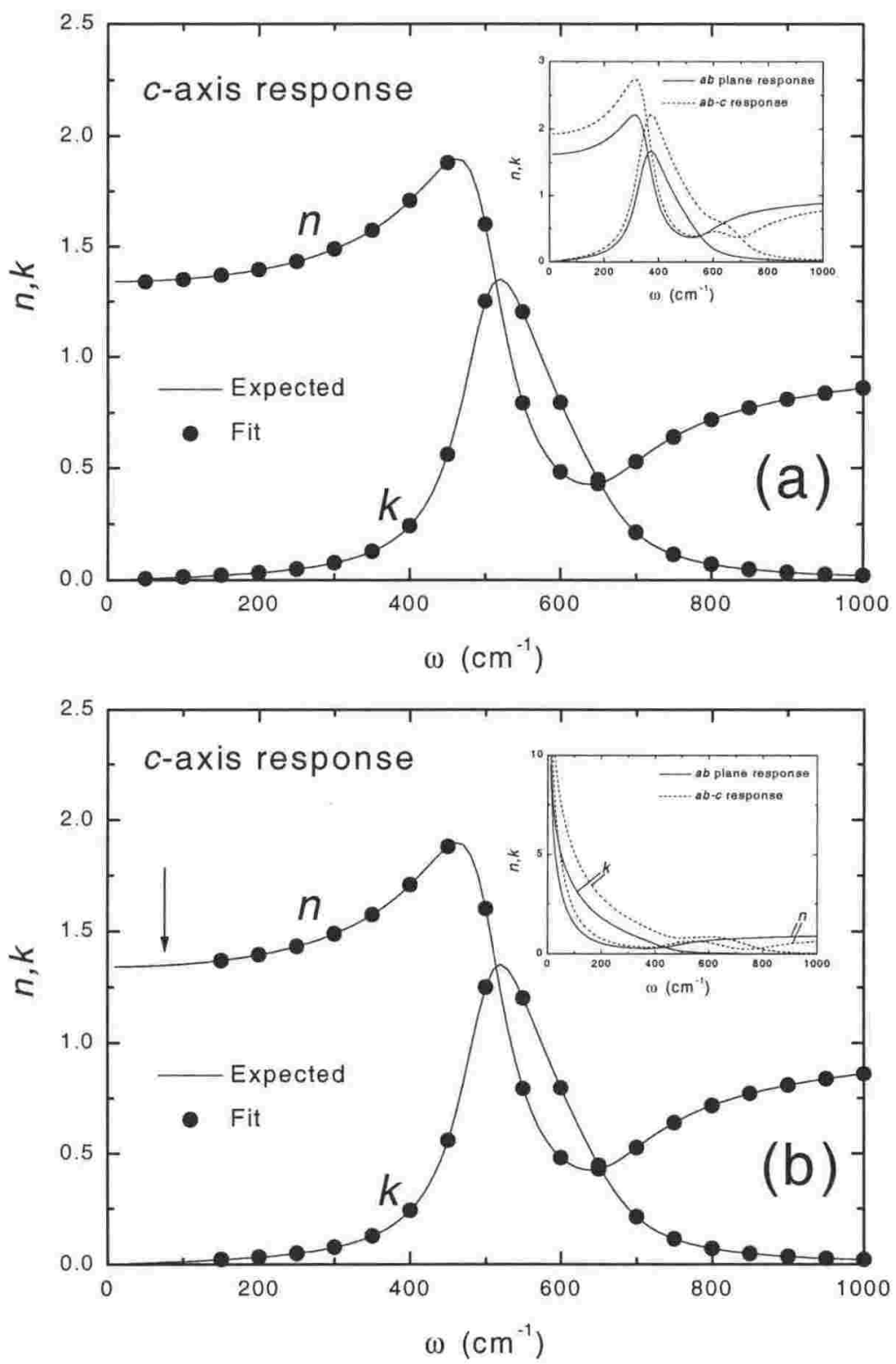

Figure B.1: The expected (solid line) and fit (solid circles) $c$-axis responses for a model anisotropic material with (a) a lorentzian $a b$ plane component and (b) a Drude $a b$ plane component, as depicted by solid lines in the insets to the respective panels. The dashed lines in the insets display the mixed $a b-c$ response. Note the arrow, indicating missing fit points, at the low frequency end of the data in panel (b). 
that solution was the correct one. However, for $\omega<150 \mathrm{~cm}^{-1}$, where the Drude component is strong, the $c$-axis response extraction routine could not converge on a solution. This result gives an insight into the difficulty of extracting values for the $c$-axis dielectric response when the mixing proportion, of $c$-axis response to $a b$ plane response, is small. 


\section{Appendix C}

\section{Normal Incidence vs Ellipsometry}

When two different techniques are used to find the optical constants we are compelled to compare the results from each to see how well they agree. The far-IR optical constants were obtained for $a b$ plane $\mathrm{PrSr}_{2} \mathrm{Mn}_{2} \mathrm{O}_{7}$ by both techniques, giving us a valuable check on how interchangeable normal incidence reflectance and ellipsometry measurements are, at least in this particular instance.

Fig. C.1 displays a comparison of the reflectivity (a) and $\sigma_{1}(\omega)(\mathrm{b})$ in the far-IR measured by the ellipsometry and normal incidence reflectivity techniques. In Fig. C.1(a) the normal incidence reflectance expected from ellipsometric optical constants was acquired by using equation 2.45 and calculating from $n$ and $k$ deduced in the ellipsometric reduction. For ease of viewing, the spectra have been displaced from each other the particular amounts detailed in the figure caption. It can be seen that for all three temperatures, the normal incidence reflectance measurement gives a consistently lower $R(\omega)$ value than does the ellipsometry measurement. This in turn translates to a lower conductivity at all three temperatures. Despite the vertical shift between the ellipsometry and normal incidence reflectivity data, however, the shape of the spectra is remarkably well preserved between the two techniques for both the $R(\omega)$ and the Kramers-Kronig transformed $\sigma_{1}(\omega)$. The only significant discrepancy occurs in the ellipsometric $R(\omega)$ beyond $\omega \approx 640 \mathrm{~cm}^{-1}$. The anomalous upturn (mentioned earlier in section 5.3.2), is due to errors 


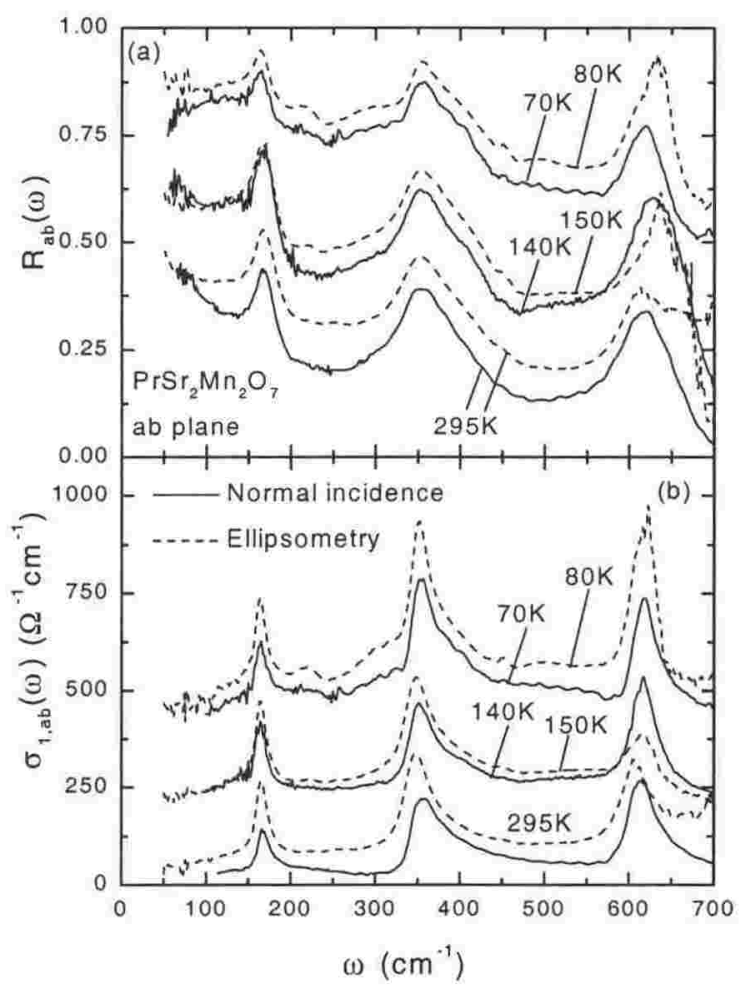

Figure C.1: Comparison in the far-IR between ellipsometry and normal incidence $R(\omega)$ (a) and $\sigma_{1}(\omega)$ (b) spectra for $a b$ plane $\operatorname{PrSr}_{2} \mathrm{Mn}_{2} \mathrm{O}_{7}$. For clarity, $R(\omega)$ for $295 \mathrm{~K}$ and $70 \mathrm{~K} / 80 \mathrm{~K}$ have been displaced respectively down and up by 0.2 and $\sigma_{1}(\omega)$ spectra for $140 / 150 \mathrm{~K}$ and $70 / 80 \mathrm{~K}$ have been displaced up by 200 and $400 \Omega^{-1} \mathrm{~cm}^{-1}$ respectively.

in the reduction process at this energy.

As seen in Fig. C.1(b), the lower reflectivity measured using normal incidence reflectance translates to a lower $\sigma_{1}(\omega)$ level for all of the spectra displayed. However, as observed for the reflectivity, the shape of the spectra is well preserved between the two techniques. The only deviation is from the ellipsometry measurements in the neighbourhood of the high-energy mode, due to the uncertainty in reduction.

A very useful advantage in knowing the ellipsometric optical conductivity is that it may be used to guide the extrapolations needed in the normal 
incidence measurement. The extrapolation used for the present dataset in Fig. C.1(b) was that of a constant $R(\omega)$ to zero frequency. It can be seen in Fig. C.1(b) that, ignoring the displacement, the low energy spectra show an agreeing trend, one not observed when a $\omega^{1 / 2}$ dependence was used in the stead of a constant one. By virtue of comparison with ellipsometry data then, the so-called Hagen-Rubens extrapolation is shown to be incompatible with the measured data.

As for answering the question of why some of the normal incidence reflectance signal is lower than the ellipsometric signal, we observe that the "loss" appears to be frequency and temperature independent. Thus it appears clear that the lower light signal is due to a greater extent of surface scattering. Indeed, although the normal incidence measurement was performed on the same cleaved surface of the $\mathrm{PrSr}_{2} \mathrm{Mn}_{2} \mathrm{O}_{7}$ sample as the ellipsometry measurement, it was performed some months later. It seems likely that surface degradation may have occurred over that time.

Another source of uncertainty, worth mentioning here, is in the degree of uniformity of the surface on a larger scale, from the viewpoint of the incident light. In the normal incidence measurement, the sample was probed with a beam of approximately $1 \mathrm{~mm}$ in diameter. The reflected spectrum varied significantly for the beam on different parts of the surface. The beam used in the ellipsometric measurement illuminated most of the $3 \mathrm{~mm} \times 4 \mathrm{~mm}$ sample surface, the reflected signal collected having interacted with a large portion of the surface. That one technique probes a small, selected area, where the other probes most of the surface, certainly must be a cause for attention. However, no attempt has been made to investigate these aspects further, and the discussion is thus now brought to a close. 


\section{Bibliography}

[1] Mastoshi Imada, Atsushi Fujimori, and Yoshinori Tokura. Metalinsulator transitions. Rev. Mod. Phys., 70:1039-1263, 1998.

[2] J. Orenstein and A. J. Millis. Advances in the physics of hightemperature superconductivity. Science, 288:468-474, 2000.

[3] T. A. Skotheim, R. L. Ausenbaumer, and J. R. Reynolds (editors). Handbook of conducting polymers. Marcel Dekker, Inc., New York, 1998.

[4] Y. Tokura and N. Nagaosa. Colossal magnetoresistive manganites. $J$. Magn. Magn. Mats., 200:1-23, 1999.

[5] J. G. Bednorz and K. A. Müller. Possible high $T_{c}$ superconductivity in the Ba-La-Cu-O system. Z. Phys. B, 64:189, 1986.

[6] Y. Moritomo, A. Asamitsu, H. Kuwahara, and Y. Tokura. Giant magnetoresistance of manganese oxides with a layered perovskite structure. Nature, 380:141, 1996.

[7] C. Zener. Interaction between the d-shells in the transition metals II. ferromagnetic compounds of manganese with perovskite structure. Phys. Rev., 82:403, 1951.

[8] P. W. Anderson and H. Hasegawa. Considerations on Double Exchange. Phys. Rev., 100:675, 1955.

[9] P. G. de Gennes. Effects of Double Exchange in magnetic crystals. Phys. Rev., 118:141, 1960. 
[10] C. W. Searle and S. T. Wang. Studies of the ionic ferromagnet (LaPb) $\mathrm{MnO}_{3}$. Can. J. Phys., 47:2703, 1969.

[11] Kenn Kubo and Nagao Ohata. A quantum theory of double exchange. I . J. Phys. Soc. Jpn., 33:21, 1972.

[12] R. M. Kusters, J. Singleton, D. A. Keen, R. McGreevy, and W. Hayes. Magnetoresistance measurements on the magnetic semiconductor $\mathrm{Nd}_{0.5} \mathrm{~Pb}_{0.5} \mathrm{MnO}_{3}$. Physica B, 155:362-365, 1989.

[13] Ken ichi Chahara, Toshiyuki Ohno, Masahiro Kasai, and Yuzoo Kozono. Magnetoresistance in magnetic manganese oxide with intrinsic antiferromagnetic spin structure. App. Phys. Lett., 63:1990, 1993.

[14] R. von Helmolt, J. Wecker, B. Holzapfel, L. Schultz, and K. Samwer. Giant negative magnetoresistance in perovskitelike $\mathrm{La}_{2 / 3} \mathrm{Ba}_{1 / 3} \mathrm{MnO}_{x}$ ferromagnetic films. Phys. Rev. Lett., 71:2331, 1993.

[15] S. Jin, T. H. Tiefel, M. McCormack, R. A. Fastnacht, R. Ramesh, and L. H. Chen. Thousandfold change in resistivity in magnetoresistive LaCa-Mn-O films. Science, 264:413, 1994.

[16] Josep Fontcuberta. Colossal magnetoresistance. Phys. World, pages 33-38, 1999.

[17] Peter M. Levy. Giant magnetoresistance in magnetic layered and granular materials. Sol. State Phys., 47:367, 1994.

[18] P. Schiffer, A. P. Ramirez, W. Bao, and S.-W. Cheong. Low temperature magnetoresistance and the magnetic phase diagram of $\left(\mathrm{La}_{1-x} \mathrm{Ca}_{x} \mathrm{MnO}_{3}\right.$. Phys. Rev. Lett., 75:3336, 1995.

[19] C. D. Ling, J. E. Millburn, J. F. Mitchell, D. N. Argyriou, J. Linton, and H. N. Bordallo. Interplay of spin and orbital ordering in the layered colossal magnetoresistance manganite $\mathrm{La}_{2-2 x} \mathrm{Sr}_{1+2 x} \mathrm{Mn}_{2} \mathrm{O}_{7}$. Phys. Rev . $B, 62: 15096,2000$. 
[20] Y. Tokura and N. Nagaosa. Orbital physics in transition-metal oxides. Science, 288:462-467, 2000.

[21] Akira Takazawa, Hiroyuki Nojiri, Keigo Nagasaka, Kazuma Hirota, and Yasuo Endoh. Investigation of phonon anomaly in the orbital order state of $\mathrm{La}_{1-x} \mathrm{Sr}_{x} \mathrm{MnO}_{3}(x \approx 1 / 8)$. Phys. Rev. B, 62:13864-13867, 2000.

[22] K. H. Kim, J. Y. Gu, H. S. Choi, G. W. Park, and T. W. Noh. Frequency shifts of the internal phonon modes in $\mathrm{La}_{0.7} \mathrm{Ca}_{0.3} \mathrm{MnO}_{3}$. Phys. Rev. Lett., 77:1877-1880, 1996.

[23] J. H. Jung, K. H. Kim, H. J. Lee, T. W. Noh, E. J. Choi, and Y. Chung. Determination of the $\mathrm{LaMnO}_{3}$ band structure based on optical studies of $\mathrm{La}_{1-x} \mathrm{Ca}_{x} \mathrm{MnO}_{3}$. J. Korean Phys. Soc., 31:L549-L552, 1997.

[24] T. Arima and Y. Tokura. Optical study of electronic structure in perovskite-type $\mathrm{RMO}_{3}(\mathrm{R}=\mathrm{La}, \mathrm{Y} ; \mathrm{M}=\mathrm{Sc}, \mathrm{Ti}, \mathrm{V}, \mathrm{Cr}, \mathrm{Mn}, \mathrm{Fe}, \mathrm{Co}, \mathrm{Ni}$, Cu). J. Phys. Soc. Jpn., 64:2488-2501, 1995.

[25] Y. Okimoto, T. Katsufuji, T. Ishikawa, T. Arima, and Y. Tokura. Variation of electronic structure in $\mathrm{La}_{1-x} \mathrm{Sr}_{x} \mathrm{MnO}_{3}(0 \leq x \leq 0.3)$ as investigated by optical conductivity spectra. Phys. Rev. B, 55:4206, 1997.

[26] T. Ishikawa, K. Tobe, T. Kimura, T. Katsufuji, and Y. Tokura. Optical study on the doping and temperature dependence of the anisotropic electronic structure in bilayered manganites: $\mathrm{La}_{1.2} \mathrm{Sr}_{1.8} \mathrm{Mn}_{2} \mathrm{O}_{7}(0.3 \leq$ $x \leq 0.5)$. Phys. Rev. B, 62:12354, 2000.

[27] J. H. Jung, K. H. Kim, D. J. Eom, T. W. Noh, E. J. Choi, J. Yu, Y. S. Kwon, and Y. Chung. Determination of electronic band structures of $\mathrm{CaMnO}_{3}$ and $\mathrm{LaMnO}_{3}$ using optical-conductivity analyses. Phys. Rev. $B, 55: 15489-15493,1997$.

[28] J. H. Jung, K. H. Kim, T. W. Noh, E. J. Choi, and J. Yu. Midgap states of $\mathrm{La}_{1-x} \mathrm{Ca}_{x} \mathrm{MnO}_{3}$ : Doping-dependent optical-conductivity studies. Phys. Rev. B, 57:R11043-R11046, 1998. 
[29] M. Quijada, J. Cerne, J. R. Simpson, H. D. Drew, K. H. Ahn, A. J. Millis, R. Shreekala, R. Ramesh, M. Rajeswari, and T. Venkatesan. Optical conductivity of manganites: Crossover from Jahn-Teller small polaron to coherent transport in the ferromagnetic state. Phys. Rev. B, 58:16093, 1998.

[30] Koshi Takenaka, Kenji Iida, Yuko Sawaki, Shunji Sugai, Yutaka Moritomo, and Arao Nakamura. Optical reflectivity spectra measured on cleaved surfaces of $\mathrm{La}_{1-x} \mathrm{Sr}_{x} \mathrm{MnO}_{3}$ : Evidence against extremely small Drude weight. J. Phys. Soc. Jpn, 68:1828-1831, 1999.

[31] Y. Moritomo, A. Machida, K. Matsuda, M. Ichida, and A. Nakamura. Magnetisation dependent behaviors of interband transitions between the exchange-split bands in doped manganite films. Phys. Rev. B, 56:5088, 1997.

[32] H. J. Lee, J. H. Jung, Y. S. Lee, J. S. Ahn, T. W. Noh, K. H. Kim, and S-W. Cheong. Optical properties of a $\mathrm{Nd}_{0.7} \mathrm{Sr}_{0.3} \mathrm{MnO}_{3}$ single crystal. Phys. Rev. B, 60:5251, 1999.

[33] O. Ripeka Mercier, R. G. Buckley, A. Bittar, H. J. Trodahl, E. M. Haines, J. Metson, and Y. Tomioka. Annealing effects on the optical conductivity of single crystal $\mathrm{La}_{1-x} \mathrm{Ca}_{x} \mathrm{MnO}_{3},(x=0.1,0.265)$. Phys. Rev. B, 64:035106, 2001.

[34] K. Takenaka, Y. Sawaki, R. Shiozaki, and S. Sugai. Electronic structure of the double-exchange ferromagnet $\mathrm{La}_{0.825} \mathrm{Sr}_{0.175} \mathrm{MnO}_{3}$ studied by optical reflectivity. Phys. Rev. B, 62:13864-13867, 2000.

[35] K.-L.Barth, D. Böhme, K. Kamarás, F. Keilman, and M. Cardona. Farir spectroscopic ellipsometer. Thin Solid Films, 234:314, 1993.

[36] Uwe Rossow and Wolfgang Richter. Chapter 3: Spectroscopic Ellipsometry in Optical Characterization of Epitaxial Semiconductor Layers, eds Gunther Bauer and Wolfgang Richter. Springer Verlag (Berlin, Heidelberg, New York), 1996. 
[37] R. M. A. Azzam and N. M. Bashara. Ellipsometry and Polarized Light. Elsevier North-Holland, 1977.

[38] M. Reedyk and T. Timusk. Evidence for $a-b$ plane coupling to longitudinal $c$-axis phonons in high- $T_{c}$ superconductors. Phys. Rev. Lett., 69:2705, 1992

[39] S. Logosthetidis, M. Cardona, P. Lautenschlager, and M. Garriga. Temperature dependence of the dielectric function and the interband critical points of CdSe. Phys. Rev. B, 34:2458, 1986.

[40] D. E. Aspnes. Approximate solution of ellipsometric equations for optically biaxial crystals. J. Opt. Soc. Am., 70:1275, 1980.

[41] John Lekner. Reflection ellipsometry of uniaxial crystals. J. Opt. Soc. Am., 14:1359, 1997.

[42] J. S. Toll. Causality and the dispersion relation: Logical foundations. Phys. Rev., 104:1760-1770, 1956.

[43] Frederick Wootten. Optical Properties of Solids. Academic Press, 1972.

[44] D. L. Rousseau, R. P. Bauman, and S. P. S. Porto. Normal mode determination in crystals. J. Ram. Spec., 10:253, 1981.

[45] International Union of Crystallography. International Tables for X-Ray Crystallography. Kynock Press, UK, 1952.

[46] M. D. Fontana, G. Métrat, J. L. Servoin, and F. Gervais. Infrared spectroscopy in $\mathrm{KNbO}_{3}$ through the successive ferroelectric phase transitions. J. Phys. C, 17:483, 1984.

[47] V. B. Podobedov, A. Weber, D. B. Romero, J. P. Rice, and H. D. Drew. Effect of structural and magnetic transitions in $\mathrm{La}_{1-x} M_{x} \mathrm{MnO}_{3}(M=\mathrm{Sr}$, Ca) single crystals in raman scattering. Phys. Rev. B., 58:43-46, 1998. 
[48] M. N. Iliev, M. V. Abrashev, H.-G. Lee, V. N. Popov, Y. Y. Sun, C. Thomsen, R. L. Meng, and C. W. Chu. Raman spectroscopy of orthorhombic perovskitelike $\mathrm{YMnO}_{3}$ and $\mathrm{LaMnO}_{3}$. Phys. Rev. B, 57:2872, 1998.

[49] P D. Battle, M. A. Green, N. S. Laskey, J. E. Millburn, P. G. Radaelli, M. J. Rosseisnsky, S. P. Sullivan, and J. F. Vente. Crystal and magnetic structures of the colossal magnetoresistance manganates $\mathrm{Sr}_{2-x} \mathrm{Nd}_{1+x} \mathrm{Mn}_{2} \mathrm{O}_{7}$ (x=0.0, 0.1). Phys. Rev. B, 54:15967, 1996.

[50] Peter R. Griffiths and James A. de Haseth. Fourier transform infrared spectrometry. Chemical Analysis Monographs: Wiley-Interscience publishers, 83, 1997.

[51] Benedict Chapman. Conducting Polymers: IR and Raman Studies. PhD thesis, Victoria University of Wellington, 1999.

[52] Jean. M. Bennett and E. J. Ashley. Infrared reflectance and emittance of silver and gold evaporated in ultrahigh vacuum. Appl. Opt., 4:221, 1965.

[53] Walter G. Driscoll and William Vaughan Eds. Handbook of Optics. McGraw Hill, 1978.

[54] J. Kircher, R. Henn, M. Cardona, P. L. Richards, and G. P. Williams. Far-infrared ellipsometry using synchrotron radiation. J. Opt. Soc. Am., 14:705, 1997.

[55] Ralf Henn. Untersuchung infrarotaktiver Phononen und freier Ladungsträger in Hochtemperatursupraleitern mittels FerninfrarotEllipsometrie. $\mathrm{PhD}$ thesis, Max-Planck Institut für Festkörperforschung, 1997.

[56] G. P. Williams. The initial scientific program at the NSLS infrared beamline. Nucl. Instrum. Methods, A291:8, 1990.

[57] N. F. M. Henry, H. Lipson, and W. A. Wooster. The Interpretation of X-Ray Diffraction Photographs. MacMillan and Co. Limited, London. 
[58] David Cochrane. Private communication. Industrial Research Ltd, 1998.

[59] G. Balakrishnan, M. R. Lees, and D. McK. Paul. Single-crystal growth and properties of the double-layered manganese oxides. Chem. Mater., 9, 1997 .

[60] A. Chattopadhyay, A. J. Millis, and S. Das. Sarma. Optical spectral weights and the ferromagnetic transition temperature of colossalmagnetoresistance manganites: Relevance of double exchange to real materials. Phys. Rev. B, 61:10738, 2000.

[61] K. Takenaka, Y. Sawaki, and S. Sugai. Incoherent-to-coherent crossover of optical spectra in $\mathrm{La}_{0.825} \mathrm{Sr}_{0.175} \mathrm{MnO}_{3}$ : Temperature-dependent reflectivity spectra measured on cleaved surfaces. Phys. Rev. B, 60:1301113015, 1999.

[62] Yasuhide Tomioka. Resistivity curves of $\mathrm{La}_{1-x} \mathrm{Ca}_{x} \mathrm{MnO}_{3}$. Private communication, 1998.

[63] T. Ishikawa, T. Kimura, T. Katsufuji, and Y. Tokura. Optical probe of anisotropic and incoherent charge dynamics in a layered ferromagnet: $\mathrm{La}_{1.2} \mathrm{Sr}_{1.8} \mathrm{Mn}_{2} \mathrm{O}_{7}$. Phys. Rev. B, 57:R8079, 1998.

[64] A. V. Boris, N. N. Koveleva, A. V. Bazhenov, P. J. M. van Bentum, Th. Rasing, S-W. Cheong, A. V. Samoilov, and N.-C. Yeh. Infrared studies of a $\mathrm{La}_{0.67} \mathrm{Ca}_{0.33} \mathrm{MnO}_{3}$ single crystal: Optical magnetoconductivity in a half-metallic ferromagnet. Phys. Rev. B, 59, 1999.

[65] K. H. Kim, J. H. Jung, and T. W. Noh. Polaron absorption in a perovskite manganite $\mathrm{La}_{0.7} \mathrm{Ca}_{0.3} \mathrm{MnO}_{3}$. Phys. Rev. Lett., 81:1517-1520, 1998.

[66] Q. Huang, A. Santoro, J. W. Lynn, R. W. Erwin, J. A. Borchers, J. L. Peng, and R. L. Greene. Structure and magnetic order in undoped lanthanum manganite. Phys. Rev. B, 55:14987, 1997. 
[67] P. G. Radaelli, G. Iannone, M. Marezio, H. Y. Hwang, S-W. Cheong, J. D. Jorgensen, and D. N. Argyriou. Structural effects on the magnetic and transport properties of perovskite $\mathrm{A}_{1-x} \mathrm{~A}_{x}{ }_{x} \mathrm{MnO}_{3}(x=0.25,0.3)$. Phys. Rev. B, 56:8265, 1997.

[68] Nubuo Furukawa. Temperature dependence of conductivity in (La,Sr) $\mathrm{MnO}_{3}$. J. Phys. Soc. Jpn., 64:3164-3167, 1995.

[69] K. H. Kim, J. H. Jung, D. J. Eom, T.W. Noh, Jaejun Yu, and E. J. Choi. Scaling behavior of spectral weight changes in perovskite manganites $\mathrm{La}_{0.7-y} \mathrm{Pr}_{y} \mathrm{Ca}_{0.3} \mathrm{MnO}_{3}$. Phys. Rev. Lett., 81:4983-4986, 1998.

[70] H. Röder, J. Zang, and A. R. Bishop. Lattice effects in the colosssalmagnetoresistance manganites. Phys. Rev. Lett., 76:135, 1996.

[71] H. J. Lee, K. H. Kim, J. H. Jung, T. W. Noh, R. Suryanarayanan, G. Dhalenne, and A. Revcolevschi. Optical studies of a layered manganite $\mathrm{La}_{1.2} \mathrm{Sr}_{1.8} \mathrm{Mn}_{2} \mathrm{O}_{7}$ : Polaron correlation effect. Phys. Rev. B, 62:11310, 2000 .

[72] Eiji Saitoh, Atsushi Asamitsu, Youichi Okimoto, and Yoshinori Tokura. Optical study of metal-insulator transition in the ferromagnetic ground state of $\mathrm{La}_{1-x} \mathrm{Sr}_{x} \mathrm{MnO}_{3}$. J. Phys. Soc. Jpn, 69:3614-3620, 2000.

[73] Kwanghee Lee, Reghu Menon, C. O. Yoon, and A. J. Heeger. Reflectance of conducting polypyrrole: Observation of the metal-insulator transition driven by disorder. Phys. Rev. B, 52:4779-4787, 1995.

[74] R. P. S. Lobo, E. Ya. Sherman, D. Racah, Y. Dagan, and N. Bontemps. Localization by disorder in the infrared conductivity of (Y,Pr) $\mathrm{Ba}_{2} \mathrm{Cu}_{3} \mathrm{O}_{7}$ films. cond-mat, 0106:555, 2001.

[75] W. Götze and P. Wölfe. Homogeneous dynamical conductivity of simple metals. Phys. Rev. B, 6:1226-1238, 1972.

[76] N. F. Mott. Metal-Insulator Transitions. Taylor and Francis, London/Philadelphia, 1990. 
[77] J. F. Mitchell, D. N. Argyriou, J. D. Jorgensen, D. G. Hinks, C. D. Potter, and S. D. Bader. Charge delocalization and structural response in layered $\mathrm{La}_{1.2} \mathrm{Sr}_{1.8} \mathrm{Mn}_{2} \mathrm{O}_{7}$ : Enhanced distortion in the metallic regime. Phys. Rev. B, 55:63, 1997.

[78] Qiming Li, Jun Zang, A. R. Bishop, and C. M. Soukoulis. Charge localization in disordered colossal-magnetoresistance manganites. Phys. Rev. B, 56:4541, 1997.

[79] L. Sheng, D. Y. Xing, D. N. Sheng, and C. S. Ting. Metal-insulator transition in the mixed-valence manganites. Phys. Rev. B, 56:R7053, 1997.

[80] P. Calvani, M. Capizzi, S. Lupi, P. Maselli, A. Paolone, and P. Roy. Polaronic optical absorption in electron-doped and hole-doped cuprates. Phys. Rev. B, 53:2756, 1996.

[81] D. N. Argyriou, J. F. Mitchell, C. D. Potter, S. D. Bader, R. Kleb, and J. D. Jorgensen. Unconventional magnetostriction in layered $\mathrm{La}_{1.2} \mathrm{Sr}_{1.8} \mathrm{Mn}_{2} \mathrm{O}_{7}$ : Evidence for spin-lattice coupling above $T_{c}$. Phys. Rev. B, 55, 1997.

[82] Masato Kubota, Hideki Yoshizawa, Yutaka Moritomo, Hirofumi Fujioka, Kazuma Hirota, and Yasuo Endoh. Interplay of the CE-type charge ordering in half-doped bilayer manganite $\mathrm{LaSr}_{2} \mathrm{Mn}_{2} \mathrm{O}_{7}$. J. Phys. Soc. Japan, 68:2202, 1999.

[83] J. Q. Li, Y. Matsui, T. Kimura, and Y. Tokura. Structural properties and charge-ordering transition in $\mathrm{LaSr}_{2} \mathrm{Mn}_{2} \mathrm{O}_{7}$. Phys. Rev. B, 55:63, 1997.

[84] Yutaka Moritomo, Arao Nakamura, Kenji Ohayama, Masayoshi Ohashi, and Kazuma Hirota. Layered antiferromagnetic state in $\left(\mathrm{Nd}_{1-z} \mathrm{La}_{z}\right) \mathrm{Sr}_{2} \mathrm{Mn}_{2} \mathrm{O}_{7}$. J. Phys. Soc. Japan, 68:631, 1999. 
[85] T. Kimura, Y. Tokura, J. Q. Li, and Y. Matsui. Successive structural transitions couple with magnetotransport properties in $\mathrm{LaSr}_{2} \mathrm{Mn}_{2} \mathrm{O}_{7}$. Phys. Rev. B, 58:11081, 1998.

[86] Tapan Chatterji, G. J. McIntyre, W. Caliebe, R. Suryanarayanan, G. Dhalenne... and A. Revcolevschi_, Bepentrant hehaving if the sharge 
[93] A. J. Millis, R. Mueller, and Boris I. Shraiman. Fermi-liquid-to-polaron crossover. I. general results. Phys. Rev. B, 54:5389-5404, 1996.

[94] A. J. Millis, R. Mueller, and Boris I. Shraiman. Fermi-liquid-to-polaron crossover. II. double exchange and the physics of colossal magnetoresistance. Phys. Rev. B, 54:5405-5417, 1996.

[95] H. G. Reik. Polarons in Ionic Crystals and Polar Semiconductors, ed. J. Devreese. North-Holland, Amsterdam, 1972.

[96] D. N. Argyriou, H. N. Bordallo, B. J. Campbell, A. K. Cheetham, D. E. Cox, J. S. Gardner, K. Hanif, A. dos Santos, and G. F. Strouse. Charge ordering and phase competition in the layered perovskite $\mathrm{LaSr}_{2} \mathrm{Mn}_{2} \mathrm{O}_{7}$. Phys. Rev. B, 61:15269, 2000.

[97] H. Yoshizawa, H. Kawano, J. A. Fernandez-Baca, H. Kuwahara, and Y. Tokura. Anisotropic spin waves in a metallic antiferromagnet $\mathrm{Nd}_{0.45} \mathrm{Sr}_{0.55} \mathrm{MnO}_{3}$. Phys. Rev. B, 58:R571, 1998.

[98] Peter D. Battle, Mark A. Green, N. Scott Laskey, Nicholas Kasmir, Julie E. Millburn, Lauren E. Spring, Stuart P. Sullivan, Matthew J. Rosseinsky, and Jaap F. Vente. Control of electronic properties by lanthanide size and manganese oxidation state in the $\mathrm{Mn}^{I I I} / \mathrm{Mn}^{I V}$ Ruddlesden-Popper phases $\operatorname{Ln}_{2-x} \mathrm{Sr}_{1+x} \mathrm{Mn}_{2} \mathrm{O}_{7}$. J. Mater. Chem, 7:977, 1997.

[99] R. Henn, J. Kircher, M. Cardona, A. Wittlin, V. H. M. Dujin, and A. A. Menovsky. Far-infrared $c$-axis response of $\mathrm{La}_{1.87} \mathrm{Sr}_{0.13} \mathrm{CuO}_{4}$ determined by ellipsometry. Phys. Rev. B, 53:9353, 1996. 